Safe nautical charts require a carefully designed bathymetric resurvey policy,
especially in shallow sancy especially in shallow sandy seas that potentially have dynamic sea floor patterns, sea is a costly process with limited resources, though. We designed a method that s able to use a time series of bathymetric surveys for the estimation of sea floor dynamics. The estimates of five specific regions the Southern North Sea lead to a set of four indicators of improve the resurd to

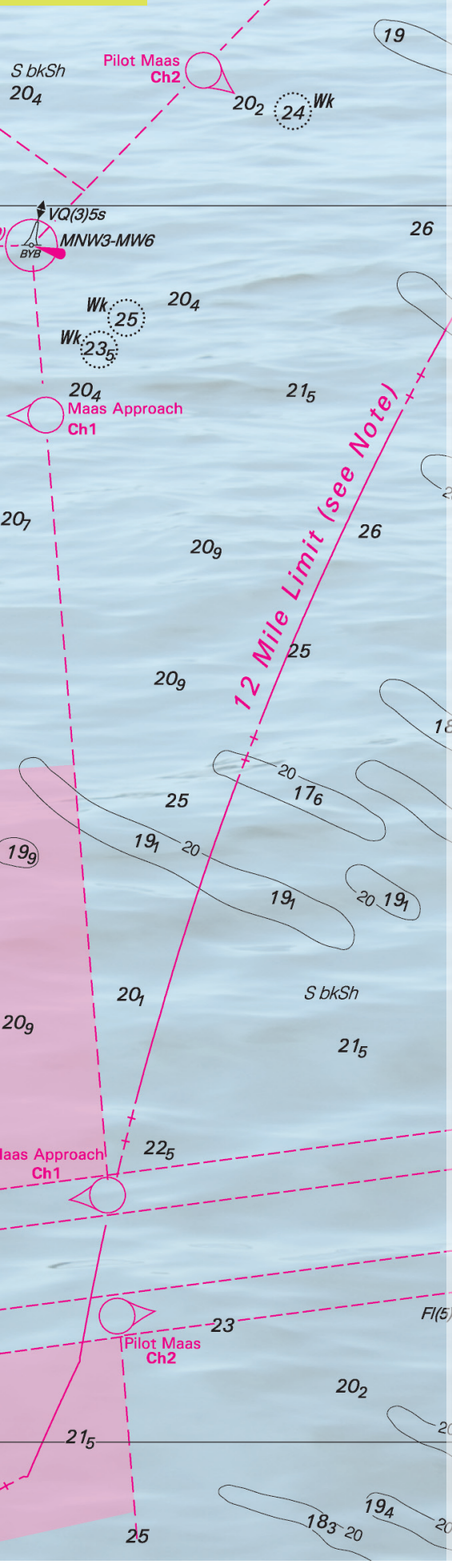

Estimating sea floor dynamics

in the Southern North Sea

to improve bathymetric survey planning

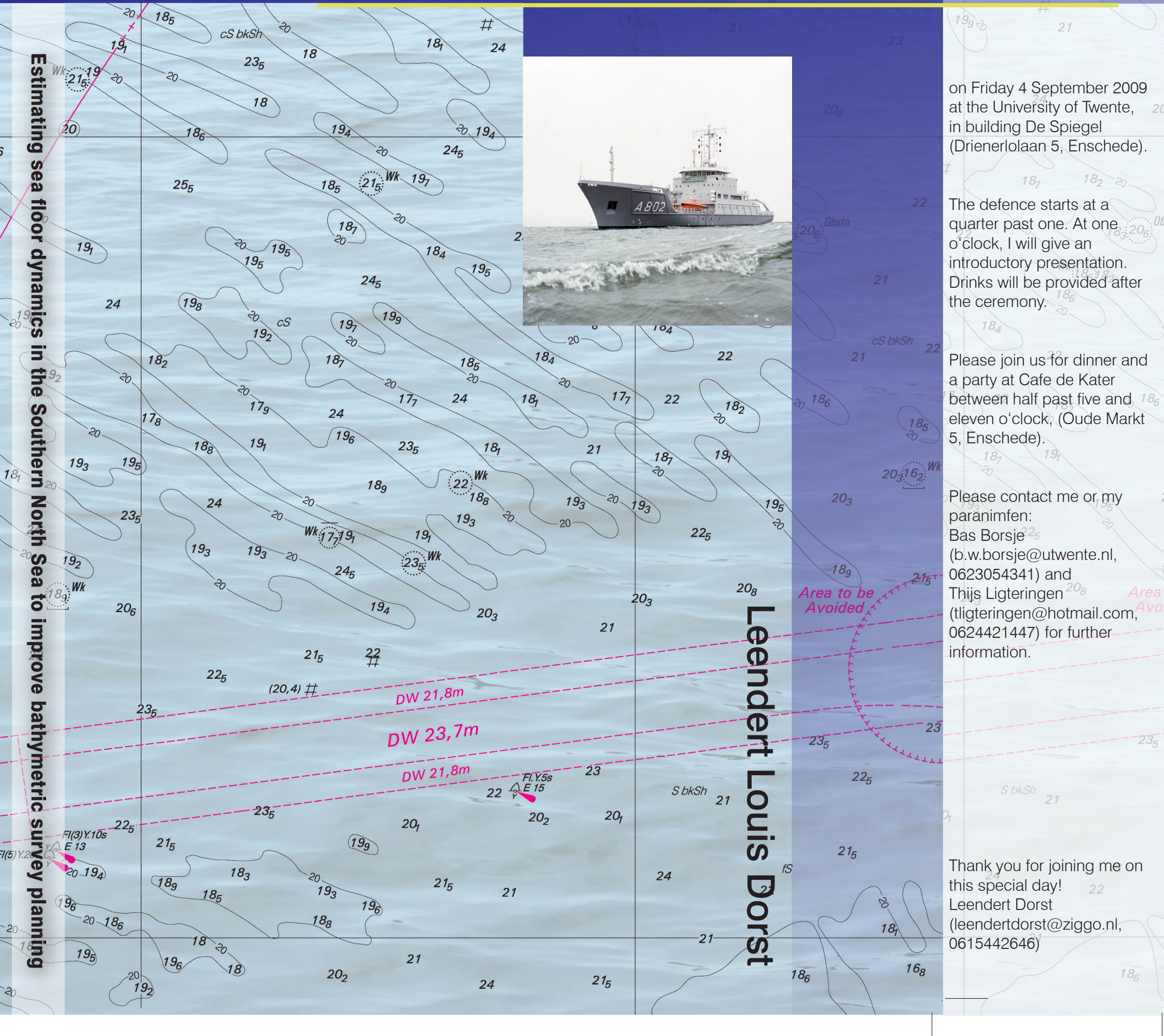

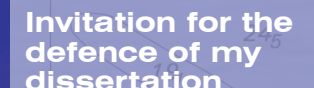

ESTIMATING SEA FLOOR SOUTHCEN NORTH SEA
O IMPROVE BATHYMEE TO IMPROVE BATHYME-
TRIC SURVEY PLANNINC 
Estimating SEA FLOOR DYNAMICS IN The Southern North SEA

TO IMPROVE BATHYMETRIC SURVEY PLANNING 
Samenstelling promotiecommissie:

prof. dr. F. Eising

prof. dr. S. J. M. H. Hulscher

dr. ir. P. C. Roos

dr. ir. C. M. Dohmen-Janssen

KTZ F. P. J. de Haan

prof. dr. ir. R. F. Hanssen

dr. R. C. Lindenbergh

prof. dr. A. Stein

prof. dr. A. Trentesaux
Universiteit Twente, voorzitter/secretaris

Universiteit Twente, promotor

Universiteit Twente, assistent-promotor

Universiteit Twente

Koninklijke Marine

Technische Universiteit Delft

Technische Universiteit Delft

ITC

Université de Lille 1

ISBN 978-90-365-2878-8

DOI: $10.3990 / 1.9789036528788$

Copyright of all contents except for the cover: (C)2009 by Leendert Louis Dorst Copyright of the cover: (c)2009 by the Hydrographic Service of the Royal Netherlands Navy

Cover: detail of Netherlands nautical chart 122, edition 2008, showing the Eurogeul crossing a sand wave field; the hydrographic survey vessel HNLMS Snellius Cover design by H. van Veldhuizen

Printed by Optima Grafische Communicatie, Rotterdam.

The reproduction of this dissertation was funded by the Hydrographic Service of the Royal Netherlands Navy.

This dissertation is published under the same title in the series Publications on Geodesy 69, Netherlands Geodetic Commission, Delft, the Netherlands, with ISBN 9789061323112.

More information: info@ncg.knaw.nl, www.ncg.knaw.nl 


\title{
ESTIMATING SEA FLOOR DYNAMICS \\ IN THE SOUthern North SEA \\ TO IMPROVE BATHYMETRIC SURVEY PLANNING
}

\author{
PROEFSCHRIFT
}

\begin{abstract}
ter verkrijging van
de graad van doctor aan de Universiteit Twente, op gezag van de rector magnificus, prof. dr. H. Brinksma, volgens besluit van het College voor Promoties

in het openbaar te verdedigen

op vrijdag 4 september 2009 om 13.15 uur
\end{abstract}

door

Leendert Louis Dorst

geboren op 10 mei 1975

te IJsselstein, provincie Utrecht 
Dit proefschrift is goedgekeurd door:

prof. dr. S. J. M. H. Hulscher, promotor

dr. ir. P. C. Roos, assistent-promotor 
to Erica Lynn, you know why 



\section{Contents}

$\begin{array}{ll}\text { Preface } & 11\end{array}$

$\begin{array}{ll}\text { Summary } & 13\end{array}$

$\begin{array}{ll}\text { Samenvatting } & 15\end{array}$

1 Introduction $\quad 17$

1.1 Nautical charting . . . . . . . . . . . . . . . . . . 17

1.2 Survey plan design . . . . . . . . . . . . . . . . . 17

1.3 Detection of sea floor dynamics . . . . . . . . . . . . . 20

1.4 Tidal sand waves . . . . . . . . . . . . . . . . 21

1.5 The uncertainty of depth measurements . . . . . . . . . . 21

1.6 Problem formulation . . . . . . . . . . . . . . . . . 23

1.7 Research question and subquestions _. . . . . . . . . . 24

1.8 Research strategy and outline . . . . . . . . . . . . . 25

2 Bathymetric applications of Geostatistics 27

2.1 Introduction . . . . . . . . . . . . . . . 27

2.2 Depth as a trend and its residuals . . . . . . . . . 28

2.2.1 The trend described by basis functions . . . . . . . . 28

2.2 .2 The residuals described by covariances . . . . . . . . 28

2.2 .3 Stationarity . . . . . . . . . . . . . . . 30

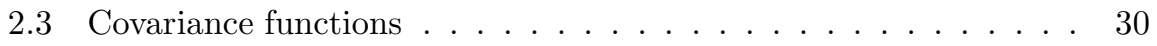

2.3.1 Definition and properties . . . . . . . . . . 30

2.3.2 Influences of measurement errors . . . . . . . . . . . 31

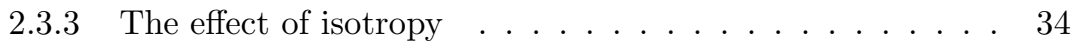

2.3.4 The empirical covariance function $\ldots \ldots \ldots \ldots . \ldots 35$

2.3 .5 Usage in hydrography . . . . . . . . . . . . . . . . . . . . . 43

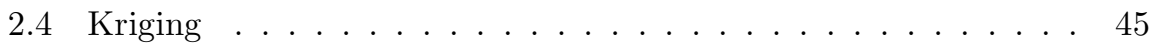

2.4.1 Kriging interpolation and properties . . . . . . . . 45

2.4 .2 The calculation of weights . . . . . . . . . . . . 47

2.4 .3 Kriging uncertainty . . . . . . . . . . . . . . . . . . 49

2.4.4 Kriging of a bathymetric survey . . . . . . . . . . . 52

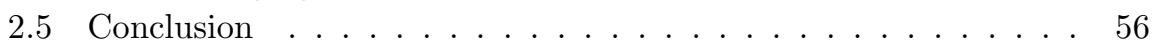


3 Estimating sea floor dynamics $\quad \mathbf{5 7}$

3.1 Introduction . . . . . . . . . . . . . . . . . . 57

3.2 The method for the estimation of sea floor dynamics . . . . . . . 59

3.2 .1 Introduction . . . . . . . . . . . . . . . . 59

3.2 .2 Input . . . . . . . . . . . . . . . . . 59

3.2.3 Sea floor characterization and its residuals . . . . . . . . 60

3.2.4 Morphological characterization . . . . . . . . . . . 61

3.2.5 Morphological analysis . . . . . . . . . . . . . 65

3.2.6 Variance and covariance estimation . . . . . . . . . . . 68

3.3 Specification of a test scenario . . . . . . . . . . . . . . . 69

3.3.1 Specification of depth values . . . . . . . . . . . . 69

3.3.2 Specification of uncertainty . . . . . . . . . . . 73

3.4 Analysis results of a dynamic sea floor . . . . . . . . . . . . . 73

3.4.1 Results of step 1, the spatial analysis . . . . . . . . . . 73

3.4.2 Results of step 2, the temporal analysis . . . . . . . . . . 74

3.5 Discussion . . . . . . . . . . . . . . . . . . 82

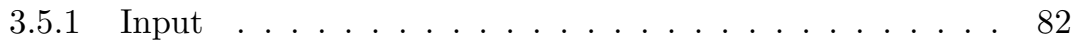

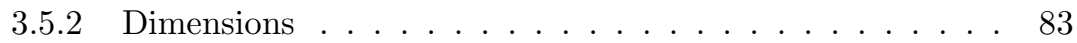

3.5.3 Interfering patterns . . . . . . . . . . . . . . . . 83

3.5.4 Design of the testing procedure . . . . . . . . . . 84

3.5.5 Design of the morphological representation and dispersion 84

3.5.6 Relation to morphodynamic models . . . . . . . . . . 85

3.6 Conclusion . . . . . . . . . . . . . . . . 86

3.A Error characteristics . . . . . . . . . . . . . . . 86

3.B Transformation of the sand wave parameters . . . . . . . . 87

3.C The application of statistical estimation and testing . . . . . . 89

3.C.1 Introduction . . . . . . . . . . . . . . . . . 89

3.C.2 Parameter estimation ............... 89

3.C.3 Extension testing . . . . . . . . . . . . . . . . . 90

3.C.4 Subsequent acceptance of extensions . . . . . . . . . 91

3.C.5 The overall test and the final estimation . . . . . . . . . . 91

3.C.6 Definition of minimal detectable biases . . . . . . . . . . . 93

3.C.7 Levels of significance for one degree of freedom . . . . . . 93

3.C.8 Levels of significance for several degrees of freedom . . . . 94

3.D The estimation of covariances . . . . . . . . . . . . . 95

3.D.1 A covariance matrix . . . . . . . . . . . . . . . 95

3.D.2 A covariance function . . . . . . . . . . . . . 96

3.D.3 A covariance matrix in step 1 for a single survey . . . . . 97

3.D.4 A covariance matrix in step 1 for all surveys . . . . . . . . 98

3.D.5 A covariance matrix in step 2 . . . . . . . . . . . . . 99

4 The analysis of migrating tidal sand waves 101

4.1 Introduction . . . . . . . . . . . . . . . . . . . . 101

4.2 The application of deformation analysis . . . . . . . . . . . 102

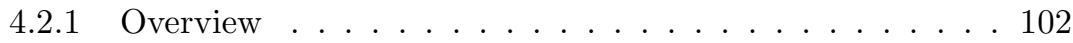

4.2.2 Bathymetric data .................. 107 
4.2 .3 Error modeling . . . . . . . . . . . . . . . . . . 107

4.3 Results of the deformation analysis . . . . . . . . . . 109

4.3.1 General results for the subareas . . . . . . . . . . . . 109

4.3.2 Detailed results for subarea H . . . . . . . . . . . . . 112

4.4 Discussion of results . . . . . . . . . . . . . . . 115

4.4.1 Correlations with spatial parameters . . . . . . . . . 116

4.4 .2 Comparison with a sand wave model . . . . . . . . . . 119

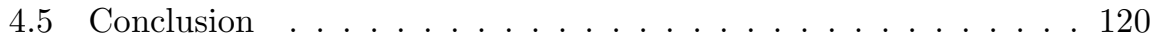

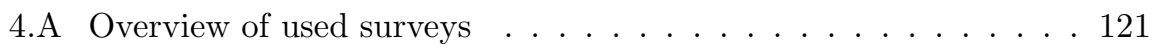

4.B Quantification of measurement errors . . . . . . . . . . . 122

4.B.1 Covariance function of the error . . . . . . . . . . 122

4.B.2 Variances of the SBES surveys . . . . . . . . . . . . 123

4.B.3 Variances of the MBES survey . . . . . . . . . . . . 124

5 Spatial variations in sea floor dynamics 127

5.1 Introduction . . . . . . . . . . . . . . . . . . 127

5.2 Deformation analysis . . . . . . . . . . . . . . . . 129

5.2 .1 Overview . . . . . . . . . . . . . . . . . . . . . 129

5.2 .2 Wavelength . . . . . . . . . . . . . . . . 133

5.3 The Selected Track region: little dynamics . . . . . . . . . . . 134

5.3.1 Spatial characterization . . . . . . . . . . . . . . . 134

5.3.2 Temporal characterization . . . . . . . . . . . . . . . 138

5.4 The Noordhinder region: comparison with other methods . . . 142

5.4 .1 Spatial characterization . . . . . . . . . . . . . 143

5.4 .2 Temporal characterization . . . . . . . . . . . . . . . 143

5.4.3 Sand wave migration and trend analysis in literature . . . 148

5.5 The region West of IJmuiden: sand wave migration . . . . . . . 152

5.5.1 Spatial and temporal characterizations . . . . . . . . 152

5.5.2 Correlations between migration and morphology . . . . . 155

5.6 The region North of Terschelling: a flat sea floor . . . . . . . 158

5.7 Discussion . . . . . . . . . . . . . . . . . . . . 158

5.7 .1 Methodical . . . . . . . . . . . . . . . . 158

5.7 .2 Morphological . . . . . . . . . . . . . . . . . 160

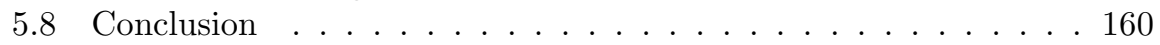

5. A Overview of used surveys $\ldots \ldots \ldots \ldots 16 \ldots \ldots$

6 Application to the resurvey policy 165

6.1 Introduction . . . . . . . . . . . . . . . 165

6.2 Background: Hydrographic practice . . . . . . . . . 168

6.2.1 Manual and automatic shoal biasing . . . . . . . . 168

6.2 .2 Inadequately surveyed areas . . . . . . . . . . . . . . . 169

6.2.3 The inclusion of sea floor dynamics in resurvey policies . 169

6.3 Method: indicators of sea floor dynamics . . . . . . . . . . . 170

6.3.1 Overview of deformation analysis . . . . . . . . . . 170

6.3.2 Domains and dimensions . . . . . . . . . . . . . . 172

6.3.3 Shallowest likely depth values . . . . . . . . . . . . . . 174 
6.3.4 Two indicators for rates of change . . . . . . . . . . 176

6.3.5 Two indicators for the risk of missed dynamics . . . . . . 177

6.3.6 Combination of the four indicators . . . . . . . . . . . . . 184

6.4 Results: indicator values for the Southern North Sea . . . . . . . 184

6.4.1 The two rates of change . . . . . . . . . . . . . . . 184

6.4.2 The two regularity parameters . . . . . . . . . . . 187

6.4.3 The two minimal detectable biases . . . . . . . . . . . 189

6.5 Results: suggested improvements . . . . . . . . . . . . . . . . . 189

6.6 Discussion . . . . . . . . . . . . . . . . . . . . . 191

6.6.1 Shallowest likely depth as a hydrographic concept . . . . 191

6.6.2 Irregularity as a morphological concept . . . . . . . . . . 191

6.6.3 The four indicators . . . . . . . . . . . . . . . . . . 192

6.7 Conclusion . . . . . . . . . . . . . . . . 192

7 Conclusions and recommendations

7.1 Answers to the subquestions . . . . . . . . . . . . . . . 195

7.2 Conclusions on the research question . . . . . . . . . . . . 197

7.3 Recommendations for further research . . . . . . . . . . . . . . . 197

7.4 Implementation at the Netherlands Hydrographic Service . . . . 200

Bibliography

Notation

About the author 


\section{Preface}

Scientific work sometimes seems to be a very individual activity. During my first scientific project, in Brussels, Belgium, for my Master's title, my supervisor taught me that in reality it is not: the ability to cooperate is a great virtue of a scientist. There are many people that have had a crucial role in the successful creation of this dissertation. Some highly valuable comments were made by anonymous reviewers, but fortunately most of the contributors are known. It is my pleasure to name a few.

This project started as an internal project at the Hydrographic Service of the Royal Netherlands Navy. Suzanne Hulscher was the first academic who saw the opportunities to turn the project into a $\mathrm{PhD}$ project. At times, it has been hard to find a balance to combine the $\mathrm{PhD}$ project with my regular work and private life. Suzanne kept supporting me, also in times of little or no progress. Thank you for providing me with this opportunity to develop myself to the person that I am today.

Soon after the initial contacts with the University of Twente, Pieter Roos became my supervisor. Pieter's perfectionism is exactly what I needed. Your comments are sincere, abundant, high-quality, and super-fast. Thank you for years of cooperation and friendship. The working environment at the Water Engineering \& Management group has been so stimulating, the group is young at heart and determined to excel. A warm thank you for all of you.

At the Hydrographic Service, the initial arrangements for the cooperation with the University of Twente were challenging: nobody was familiar with the construction of a shared promotion project. It all worked out, thanks to the constructive efforts of Ina Elema, Ruurd van Rooijen and Floor de Haan. Please accept my sincere gratitude. The main advocate of the project was Jan Appelman, from the start to the end. Jan, you are a passionate professional, someone who makes a difference. Marco Kwanten, my room mate, thank you for turning me from a geodesist into a hydrographer.

Appreciation also goes to Peter Menting and Bregt Huizenga, who I supervised on this topic. Cooperation with you helped me to understand things better myself. I highly value the curiosity and the persistence of the both of you. I am grateful to Roderik Lindenbergh, Hans Wüst and Thaïenne van Dijk for their professional interest, valuable comments and helpful discussions. The sources of the data are the Hydrographic Service and Rijkswaterstaat Noordzee. Both the organizations are gratefully acknowledged. Daniëlle van Kuijk and Simon Bicknese, thank you for assisting me. 
Gedurende het gehele project was het mogelijk om zonder verdere kosten gehuisvest te worden, voornamelijk op de Zuidkamp en op de campus van de universiteit. Hartelijk dank voor de geboden voorzieningen gaat uit naar de Koninklijke Marine, de Koninklijke Luchtmacht, de Koninklijke Landmacht en de Universiteit Twente.

De trots van mijn lieve ouders is een belangrijke inspiratiebron voor mij. Dank jullie wel voor alle kansen die jullie me hebben gegeven. Maarten, Herman, Tineke, jullie vriendschap verandert wel van aard maar niet van intensiteit. The $\mathrm{M} \stackrel{\mathrm{c}}{=}$ Cormick family is a wonderful example of joy and generosity. Thank you for your hospitality, in New York and New Jersey I found the right atmosphere to work hard and be happy. Erica, our future is bright. We are one step closer now, thanks to a truly cooperative effort. This project is as much your achievement as it is mine.

Leendert Dorst, July 28, 2009. 


\section{Summary}

Safe nautical charts require a carefully designed bathymetric survey policy, especially in shallow sandy seas that potentially have dynamic sea floor patterns. Bathymetric resurveying at sea is a costly process with limited resources, though. A pattern on the sea floor known as tidal sand waves is clearly present in bathymetric surveys, endangering navigation in the Southern North Sea because of the potential dynamics of this pattern. An important factor in an efficient resurvey policy is the type and size of sea floor dynamics. The uncertainties of measurement and interpolation associated with the depth values enable the statistical processing of a time series of surveys, using deformation analysis. Currently, there is no procedure available that satisfies the Royal Netherlands Navy requirements. Therefore, a deformation analysis procedure is designed, implemented and tested in such a way that the procedure works on bathymetric data and satisfies the Royal Netherlands Navy requirements. Also, it is necessary to develop a procedure that translates the results into changes of the resurvey policy, taking into account their confidence intervals.

To describe the sea floor statistically, we assume the sea floor to consist of a spatial trend function (or characterization) and a residual function (or dispersion). Such a description is called a representation. The covariances between positions are expressed in a covariance function, based on the residual function. The covariance function is used by Kriging, an interpolation procedure that propagates the variances and covariances of the data points to variances of the interpolated values. This approach is used widely for spatial analyses, like the interpolation of a bathymetric data set.

The method that we propose uses Kriging to produce a time series of grids of depth values and their variances. Subsequently, it uses deformation analysis, a statistical procedure based on testing theory. Our application of deformation analysis is particularly aimed at the detection of dynamics in areas with tidal sand waves, resulting in parameter estimates for the sea floor dynamics, and their uncertainty. We apply the method to sea floor representations both with and without a sand wave pattern. A test scenario is set up, consisting of a survey of an existing area in the Southern North Sea, for which dynamics are simulated. The results show that the proposed method detects different types of sea floor dynamics well, leading to satisfactory estimates of the corresponding parameters.

We show results for the anchorage area Maas West near the Port of Rotterdam, the Netherlands first. The area is divided into 18 subareas. The results show that a sand wave pattern is detected for most of the subareas, and a shore- 
ward migration is detected for a majority of them. The estimated migration rates of the sand waves are up to $7.5 \mathrm{~m} / \mathrm{yr}$, with a $95 \%$ confidence interval that depends on the regularity of the pattern. The results are in confirmation with previously observed migration rates for the Southern North Sea, and with an idealized process-based model.

Thereafter, we analyze several other areas for which a time series of surveys is available in the bathymetric archives of the Netherlands Hydrographic Service, to study the spatial variations in sea floor dynamics. We present results for several sand wave areas and a single flat area. In some of those areas, dredging takes place, to guarantee minimum depths. The results indicate sand wave migration in areas close to the coast, and bed level changes of the order of decimeters. The dominant wavelength of the sand waves varies. We compare our results to literature of the same sand wave areas, in which we find similar migration rates, and different wavelengths.

By formulating four indicators, recommendations are made for the resurvey policy on the Belgian and Netherlands Continental Shelf. These indicators follow from the estimates for sea floor dynamics. We present a concept for the shallowest likely depth surface, on which we base two of the indicators. The other two indicators act as a warning: they quantify the potentially missed dynamics, which makes the procedure more robust in case of complicated morphology. We show clear differences in recommended resurvey frequency between the five analyzed regions.

We conclude that the designed method is able to use a time series of bathymetric surveys for the estimation of sea floor dynamics in a satisfactory way. Those dynamics may be present on the scale of the sea floor, it may be a local effect, or it may be due to a tidal sand wave pattern. Also, the results are successfully reduced to a set of four indicators, used to improve a resurvey policy. Based on these conclusions, we formulate recommendations on the extrapolation of the results in space and time, on potential adaptations to the designed procedure, and on implementation of the procedure. 


\section{Samenvatting}

Veilige zeekaarten vereisen een nauwkeurig vormgegeven bathymetrisch opnemingsbeleid, met name in ondiepe, zandige zeeën die mogelijk dynamische zeebodempatronen vertonen. Bathymetrisch opnemen op zee is echter een kostbaar proces, waarvoor beperkte middelen beschikbaar zijn. Het gevaarlijkste patroon voor navigatie in de Zuidelijke Noordzee zijn de getijdezandgolven, die duidelijk aanwezig zijn in bathymetrische opnemingen, en die potentieel dynamisch gedrag vertonen. Type en grootte van zeebodemdynamiek vormen daarmee een belangrijke factor in een efficiënt opnemingsbeleidsplan. Het verbinden van meet- en interpolatieonzekerheden aan dieptewaarden maakt het mogelijk om een tijdsserie opnemingen statistisch te verwerken met deformatieanalyse. Omdat er op dit moment geen procedure beschikbaar is die aan de eisen van de Koninklijke Marine voldoet, wordt een procedure voor deformatieanalyse ontworpen, geïmplementeerd en beproefd, die afgestemd is op bathymetrische data en die aan deze eisen voldoet. Ook is het nodig om een procedure te ontwerpen om de resultaten in het opnemingsbeleidsplan op te nemen die gebruik maakt van de betrouwbaarheidsintervallen van de resultaten.

Een statistische zeebodembeschrijving veronderstelt dat deze bestaat uit een ruimtelijke trendfunctie (of karakterisering) en een restfunctie (of dispersie). Zo'n beschrijving wordt een representatie genoemd. De ruimtelijke covarianties worden uitgedrukt in een covariantiefunctie, gebaseerd op de restfunctie. Een toepassing van de covariantiefunctie is Krigen, een interpolatieprocedure die de varianties en covarianties van de data voortplanten naar varianties van de geïnterpolateerde waarden. Deze benadering wordt algemeen gebruikt voor ruimtelijke analyses, zoals de interpolatie van een bathymetrische dataset.

De voorgestelde methode gebruikt Krigen om een tijdsserie met grids van dieptewaarden en hun varianties te produceren. Vervolgens gebruikt de methode deformatieanalyse, een statistische procedure gebaseerd op toetsingstheorie. De toepassing van deformatieanalyse is in het bijzonder gericht op het vinden van dynamiek in gebieden met getijdezandgolven, resulterend in parameterschattingen voor zeebodemdynamiek en hun onzekerheid. De methode wordt toegepast op zeebodemrepresentaties met en zonder zandgolfpatroon. We testen een scenario dat bestaat uit een grid met werkelijke diepten in de Zuidelijke Noordzee, en gesimuleerde dynamiek. De resultaten tonen aan dat de voorgestelde methode de diverse typen zeebodemdynamiek goed schat, waarbij de schattingen voor de corresponderende parameters naar tevredenheid zijn. 
Eerst presenteren we resultaten voor het ankergebied Maas West nabij de haven van Rotterdam. Het gebied is daartoe opgedeeld in 18 deelgebieden. De resultaten tonen aan dat een zandgolfpatroon gedetecteerd wordt voor de meeste deelgebieden, en voor de meeste van deze deelgebieden wordt een kustwaartse migratie gevonden. De schattingen voor de snelheid van de zandgolfmigratie lopen op tot $7.5 \mathrm{~m} / \mathrm{jr}$, met een $95 \%$ betrouwbaarheidsinterval dat afhangt van de regelmaat van het patroon. De resultaten bevestigen eerder waargenomen migratiesnelheden voor de Zuidelijke Noordzee, en een geïdealiseerd procesgebaseerd model.

Vervolgens analyseren we een aantal andere gebieden waarvoor een tijdsserie opnemingen beschikbaar is in de bathymetrische archieven van de Dienst der Hydrografie, zodat de ruimtelijke variatie in zeebodemdynamiek duidelijk wordt. We presenteren resultaten voor een aantal gebieden met zandgolven, en voor één vlak gebied. Sommige van de gebieden worden door baggeren op diepte gehouden. De resultaten geven aan dat zandgolven migreren in gebieden nabij de kust, en dat diepteveranderingen plaatsvinden in de orde van grootte van decimeters. De dominante golflengte van de zandgolven varieert. We vergelijken de resultaten met de bestaande literatuur over deze zandgolfgebieden, en vinden vergelijkbare migratiesnelheden en afwijkende golflengten.

Het fomuleren van vier indicatoren maakt het mogelijk om aanbevelingen te doen voor het opnemingsbeleidsplan voor het Nederlands en Belgisch Continentaal Plat. De indicatoren volgen uit de schattingen voor zeebodemdynamiek. We presenteren het concept van het ondiepste te verwachten diepteoppervlak, waar we twee indicatoren op baseren. De beide andere indicatoren hebben een waarschuwende functie: ze kwantificeren de potentieel gemiste dynamiek, en maken daarmee de procedure robuuster voor gecompliceerde morfologie. We geven duidelijke verschillen aan in aanbevolen heropnemingsfrequentie tussen de vijf geanalyseerde regio's.

We concluderen dat de ontworpen methode in staat is naar tevredenheid een tijdsserie bathymetrische opnemingen te gebruiken voor het schatten van zeebodemdynamiek. Zulke dynamiek kan aanwezig zijn op de schaal van de zeebodem, een lokaal effect, of veroorzaakt door een patroon getijzandgolven. Ook zijn de resultaten naar tevredenheid verder gereduceerd tot vier indicatoren voor de verbetering van het opnemingsbeleidsplan. Op basis van deze conclusies formuleren we aanbevelingen om de resultaten te extrapoleren in ruimte en tijd, om aanpassingen te overwegen in de ontworpen procedure, en voor de implementatie van de procedure. 


\section{Chapter 1}

\section{Introduction}

\subsection{Nautical charting}

The Hydrographic Service of the Royal Netherlands Navy (RNLN) is the Dutch government office that is responsible for nautical surveying and charting, in order to enable safe navigation at sea. To ensure the presence of accurate information on e.g. depth on board, the usage of official nautical charts is mandatory for many types of ships. Nautical charts are based on bathymetric surveys at sea, i.e. sets of measurements of the depth of the sea floor. A bathymetric survey at sea is costly because of both the large associated expenses and the variety of complicated observations that are necessary. Another complication is that bathymetric information becomes outdated after limited time, because of the changing nature of the sea floor in many sandy shallow seas.

The sea floor of many parts of sandy shallow seas is covered by a variety of rhythmic features. From a nautical point of view, tidal sand waves, which are characterized by wavelengths of hundreds of meters and amplitudes of up to several meters, are the most relevant of these features. As shown in Figure 1.1, they are widely present on the Belgian and Netherlands Continental Shelf (BNLCS). Tidal sand waves often migrate or grow, which makes sea floor dynamics a relevant factor for resurvey planning.

\subsection{Survey plan design}

To manage bathymetric surveys efficiently, it is necessary to plan the deployment of the two hydrographic survey vessels of the RNLN according to a carefully designed survey policy. Survey policies are made worldwide [NOAA Office of Coast Survey, 2008; De Oliveira et al., 2007; Dehling, 2006; Whatrup et al., 2005]. The RNLN policy, given in Figure 1.2, assigns a resurvey frequency to all areas of the BNLCS under RNLN responsibility.

Over the last decades, several attempts have been made to quantify dynamics of the Southern North Sea to optimize the resurvey policy [Langeraar, 1966; Scheele, 1986; Velberg, 1993; De Haan, 1996; Gillissen and Pulles, 1996; Van Wijk, 2000]. Because of the complicated nature of the dynamics of the sea floor, the limited quality and availability of bathymetric data, and the large size of the data sets, those attempts have not led to significant changes. Over the past years, it has not been possible to maintain the resurvey frequencies in the 


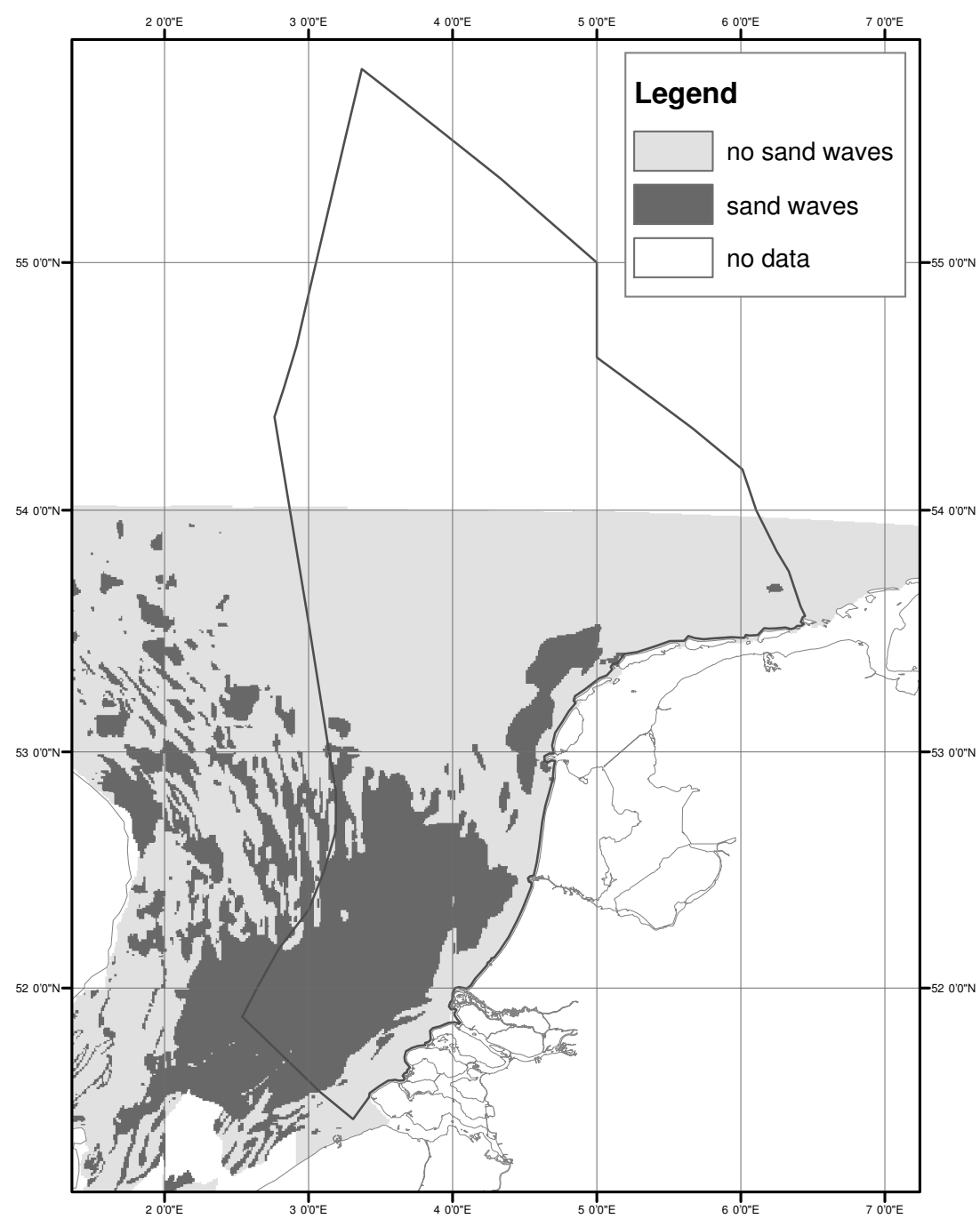

Figure 1.1: The presence of tidal sand waves in the Southern North Sea (Figure courtesy of H. H. van der Veen and B. Perez-Lapeña) 


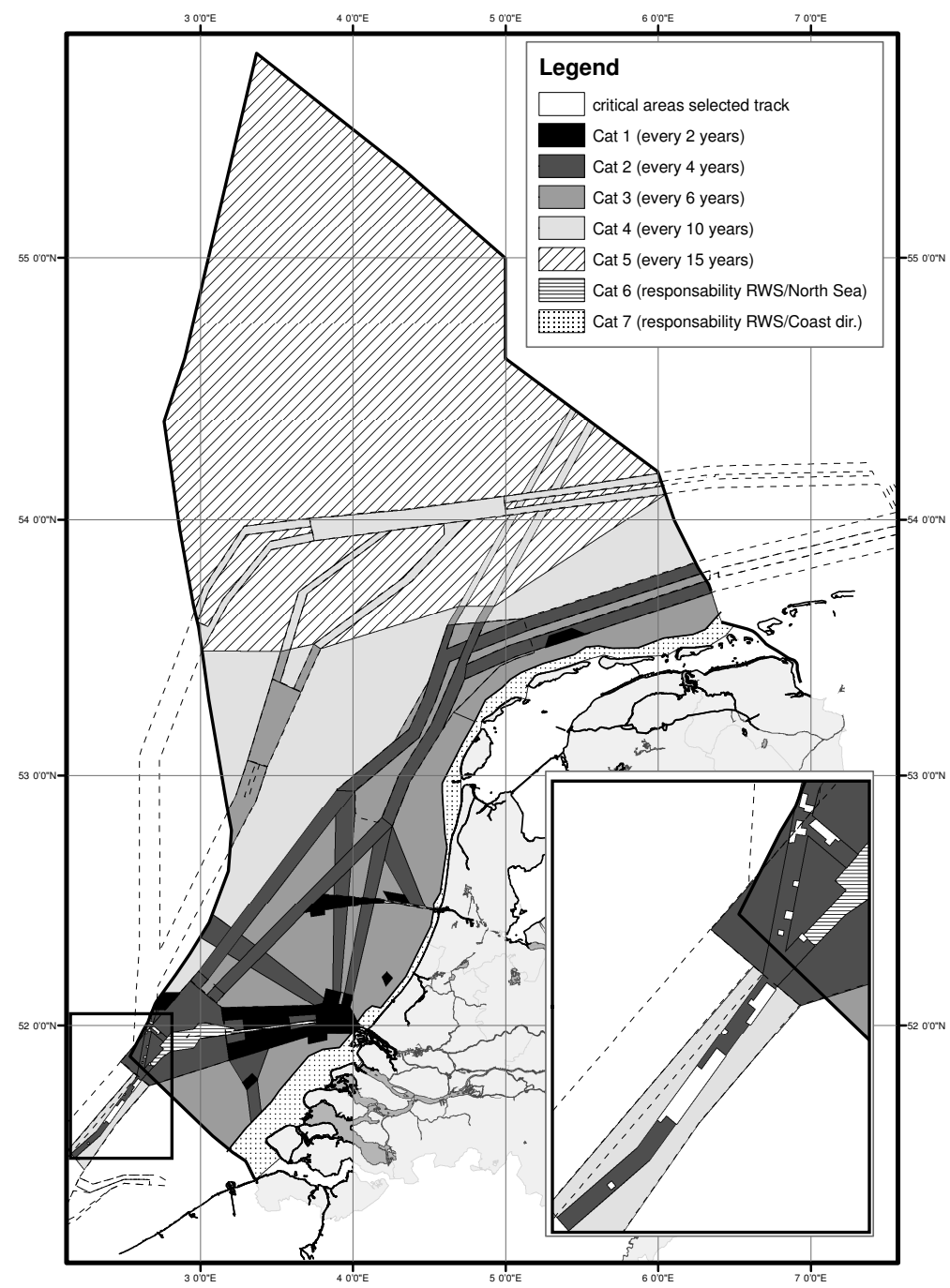

Figure 1.2: The 2003 resurvey policy of the Hydrographic Service of the Royal Netherlands Navy, for the areas of the Belgian and Netherlands Continental Shelf under its responsibility. In the so-called Selected Track for deep draught vessels on the Belgian Continental Shelf, Critical areas are defined that have a resurvey frequency of once every two years. The Hydrographic Service does not survey the areas that are under responsibility of Rijkswaterstaat North Sea, or their coastal directorates. (Figure courtesy of Lt I.J. Nijman) 
current resurvey policy, due to both technical and operational reasons. This makes reductions in frequency inevitable. A risk assessment study of the RNLN [Dienst der Hydrografie, 2007] pointed out the importance of sea floor dynamics for adaptations in resurvey policy.

\subsection{Detection of sea floor dynamics}

Various approaches exist for the analysis of a series of bathymetric surveys to detect and quantify sea floor deformations. Literature overviews have been published by Wever [2004] and Van Maren [1998]. We characterize these methods by three properties:

- number of spatial dimensions: zero-dimensional methods consider points, one-dimensional methods lines, and two-dimensional methods surfaces;

- role of time: considering discrete differences between the survey moments, or assuming continuous dynamics, like linear trends;

- interpretation of the data: stochastic estimates include a confidence interval to describe their uncertainty, while deterministic methods deliver exact values, ignoring random noise from the measurement processes and other sources.

The oldest documented analyses of a time series of bathymetric data were done by Jones et al. [1965], Salsman et al. [1966] and Langeraar [1966]. They used graphical techniques, like the comparison of crest positions, profiles, or bathymetric maps. Since then, almost all applied methods have used one or two spatial dimensions, which means that they study depth change in relation to the spatial variation of depth and its dynamics. Bowyer [1992] was the first to document a zero-dimensional analysis of the dynamics of each point of the sea floor in an area. He subtracted depth values of two surveys at equal positions.

The detection of continuous dynamics in marine morphology, instead of differences between bathymetric surveys, was first done by Lanckneus et al. [1994]. It was not until Dorst [2003] and Wüst [2004] that several surveys were used to estimate trends in a zero-dimensional analysis on grids of points. This new approach to the role of time enables the connection with the physical processes behind those dynamics.

The confrontation of deterministic results with general error models has been done since Terwindt [1971]. Moreover, Salsman et al. [1966] already used markers on the sea floor to correct for positioning errors. A next step was to subtract the a priori measurement-related variance from the total variance of depth differences between surveys. The result is considered to be the change in depth, as done by Smith [1986] and later by Velberg [1993]. However, the application of stochastic approaches, which take maximum advantage of the availability of uncertainty for each individual depth, started with Dorst [2003] and Wüst [2004]. 
Table 1.1: Offshore rhythmic bed forms in shallow seas with sandy beds, including typical wavelength, maximum height and typical migration rate.

\begin{tabular}{llll}
\hline \hline & \multicolumn{2}{l}{ wavelength max. height } & migration rate \\
\hline Ripples & $\sim 1 \mathrm{~m}$ & $0.01 \mathrm{~m}$ & $\sim 1 \mathrm{~m} /$ hour \\
Megaripples & $\sim 10 \mathrm{~m}$ & $0.1 \mathrm{~m}$ & $\sim 1 \mathrm{~m} /$ day \\
Tidal sand waves & $\sim \mathbf{5 0 0} \mathbf{~ m}$ & $\mathbf{5} \mathbf{~ m}$ & $\sim \mathbf{1 0} \mathbf{~ m} /$ year \\
Long bed waves & $\sim 1.5 \mathrm{~km}$ & $5 \mathrm{~m}$ & unknown \\
Tidal sand banks & $\sim 6 \mathrm{~km}$ & $10 \mathrm{~m}$ & $\sim 1 \mathrm{~m} /$ year \\
\hline \hline
\end{tabular}

\subsection{Tidal sand waves}

Rhythmic morphological patterns are abundant in shallow seas with sandy beds like the Southern North Sea, as seen in Figure 1.1. Changes in their height and position have been reported frequently [Wright, 1992; Terwindt, 1971], possibly endangering navigation through busy shipping lanes. Such patterns exist on several scales [Knaapen et al., 2001; Knaapen, 2004] that are given in Table 1.1. We focus on tidal sand waves, as they form the only pattern that could endanger navigation at sea, due to the combination of their significant height and considerable migration rate. An example of their presence is given in Figure 1.3, for the approach area of the Port of Rotterdam, one of the busiest ports in the world. This port is visited by ships with draughts of over $20 \mathrm{~m}$.

The properties of tidal sand waves have been explained using idealized process-based models. Hulscher [1996] showed that the formation of tidal sand waves can be explained as an instability of a horizontal, sandy seabed subject to tidal flow and a sediment transport mechanism. Such a linear stability analysis typically leads to preferred values of the wavelength and the growth rate. Later on, the physics of this model have been refined in various respects. For example, sand wave migration can be explained quantitatively as resulting from the specification of a residual current [Németh et al., 2002] or a higher harmonic to the tidal forcing [Besio et al., 2004]. A recent extension of the above approach leads to a nonlinear model capable of describing tidal sand waves in an equilibrium state [Sterlini et al., 2009]. Besio et al. [2008] provide an overview of process-based sand wave models.

\subsection{The uncertainty of depth measurements}

For the analysis of any set of measured data, it is essential to realize that each measurement is subject to a measurement error. The resulting uncertainty of the measurement is usually given at a specified confidence level, based on a statistical variance. The maximum acceptable uncertainties due to depth measurement errors have been specified by the IHO S44 standards [International Hydrographic Organization, 2008a]. This maximum uncertainty is usually depth dependent, see Wells and Monahan [2002] for an overview of these and other 


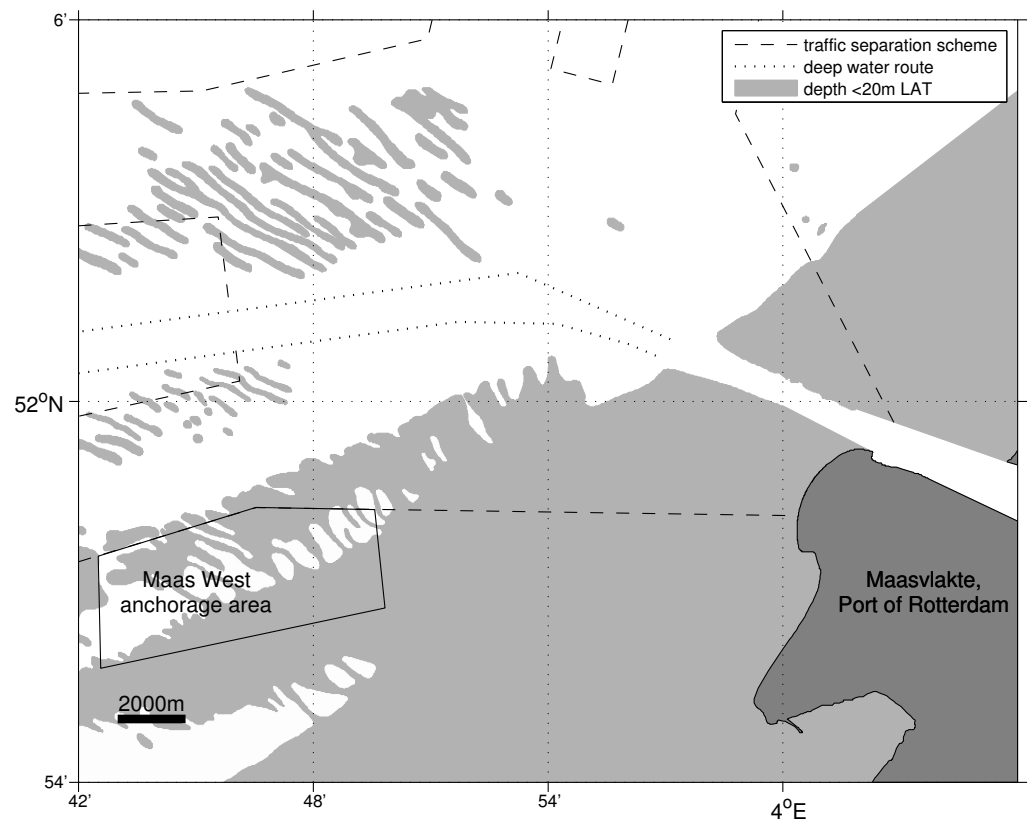

Figure 1.3: The region West of the Port of Rotterdam, which is intensively used by ships with a limited under keel clearance. The sandy shallow sea floor shows a variety of rhythmic patterns. The shaded areas, bounded by the $20 \mathrm{~m}$ depth contour, clearly show tidal sand waves, in Southeast/Northwest direction.

standards. The S44 standards apply to cleaned data, i.e. data from which the gross errors have been removed, and the systematic errors have been corrected for. The maximum uncertainty therefore applies to random errors, and the residual effects of the systematic errors.

To formulate a variance, two approaches are possible. The a priori approach uses the uncertainties of all sensors to calculate a Total Propagated Uncertainty (TPU). These uncertainties are commonly known from sensor specifications of the manufacturer. The a posteriori approach assumes that nearby measurements should have a constant value, and therefore uses the differences to calculate a variance. A disadvantage of the a priori variance is that it relies on specifications, instead of the true performance of a sensor. A disadvantage of the a posteriori variance is that residual systematic effects are not found. Approval of a bathymetric survey should therefore both include consideration of a priori and a posteriori variances.

Over the past decade, the inclusion of variances in the storage of survey results has become common practice. This was driven by two developments. First, it has become common to outsource bathymetric survey work, which has resulted in wide use of the IHO S44 standard in survey specifications. Second, 
the worldwide introduction of multi-beam echo sounders (MBES), which produce very large data sets, has required automated methods for data processing. Therefore, algorithmic methods have been introduced that require data quality as part of their input. Examples are the navigation surface [Smith, 2003; Smith et al., 2002] and the Combined Uncertainty and Bathymetry Estimator (CUBE) [Calder, 2003].

\subsection{Problem formulation}

To produce reliable nautical charts (Section 1.1), the RNLN needs up-to-date bathymetric surveys. Sea floor dynamics in the Southern North Sea needs to be quantified in order to optimize the resurvey policy (Section 1.2). Resurvey planning is best served by applying a method that can distinguish between different types of dynamics in an area. One may e.g. wish to differentiate between trends in large-scale* sea floor depth, intermediate-scale trends in crest height of a sand wave pattern, and small-scale changes at a specific location. It is thereby necessary to separate true sea floor deformation from measurement inaccuracies, or to establish whether a new survey confirms previous surveys. This requires a method that has an adaptable number of spatial dimensions, that considers trends, that is able to analyse sand wave behavior, and that treats its input and output as stochastic estimates. None of the existing methods has these properties (Section 1.3).

Nowadays, the physical processes of morphodynamics are better understood (Section 1.4), the specification of the uncertainty of bathymetric data is common practice (Section 1.5), and information technology enables the storage and efficient analysis of large data sets. These developments make the use of a statistical method which estimates a limited set of sea floor parameters and their uncertainties desirable and feasible. This situation creates opportunities for the development of a new, statistical method.

A technique which may satisfy the RNLN requirements is Deformation analysis [Chrzanowski, 2006; Koch, 1999; De Heus et al., 1994]. This statistical method is well-known and has been widely applied for the analysis of a time series of land surveys. To make this method applicable to the analysis of a time series of bathymetric surveys, the technique has to be redesigned. This makes it possible to apply deformation analysis on a series of depth values on a regular grid, potentially showing a complicated rhythmic pattern. Deformation analysis is able to estimate trends with a specified confidence interval. Important characteristics include its flexibility in the number of dimensions, and in the number of available surveys. Moreover, it has the ability to distinguish between a static situation, trends in sea floor behaviour, and single surveys that show differing properties, known as outliers. Such a distinction is made for both large-scale depth variation and for tidal sand wave behavior.

* Large-scale and small-scale are used throughout this study in its general definition, meaning 'wide ranging' and 'limited ranging' respectively. In its cartographic definition, these meanings are reversed. 
The problem that the RNLN faces is twofold. In the first place, a deformation analysis procedure for bathymetric use should be designed, implemented and tested. The procedure should satisfy the RNLN requirements. In the second place, it is necessary to develop a procedure that includes the results of the deformation analysis procedure in the resurvey policy for the Southern North sea, taking into account the specific statistical properties of these results.

\subsection{Research question and subquestions}

To solve the formulated problem, the research question this project aims to answer is:

How can deformation analysis use a time series of bathymetric surveys to estimate sea floor dynamics that may include a migrating tidal sand wave pattern in a satisfactory way, and how can these results be applied to improve a resurvey policy?

In this project, an estimation is said to be satisfactory if it satisfies the following three requirements. (1) The method should detect all significant sea floor dynamics, potentially a combination of various types. (2) The dynamics are estimated using as few parameters as possible to provide for a clear description of the dynamics. (3) The uncertainties associated with the dynamics are as small as possible.

If the resurvey policy satisfies the following four requirements, it is said to be improved. (1) All detected sea floor dynamics have the potential to influence the policy. (2) The risk of missed sea floor dynamics is accounted for. (3) Sea floor dynamics that have a smaller uncertainty have a larger influence. (4) Sea floor dynamics that pose a more severe threat to navigation have a larger influence.

The research question is answered by considering the following set of subquestions.

Q1: How can we express a bathymetric survey as a grid of depth values and their uncertainties, to be used as input for deformation analysis?

Q2: How can deformation analysis estimate sea floor dynamics, using the appropriate sea floor parameters, based on a time series of bathymetric surveys expressed as grids of depth values and their uncertainties?

Q3: How do estimates of parameters for sand wave pattern dynamics, obtained by the application of deformation analysis, vary on the scale of such a pattern for a specific sand wave area in the Southern North Sea?

Q4: How do estimates of parameters for the sea floor and its changes, obtained by the application of deformation analysis, vary over several areas in the Southern North Sea?

Q5: How can the results of the application of deformation analysis be used to improve the resurvey policy of the Netherlands Hydrographic Service? 


\subsection{Research strategy and outline}

The first two questions are methodical. The correct expression of bathymetric survey data (Q1) is necessary as preparatory work for the estimation of sea floor change (Q2): survey data needs to be given at an appropriate resolution and with associated uncertainties. To this end, we base our design for a preparatory procedure on a literature study, in Chapter 2. With a suitable expression of the survey data, an estimation procedure is designed to estimate parameters for sea floor dynamics in a satisfactory way, as specified above, using deformation analysis, as given in Chapter 3 .

Chapter 2 presents geostatistical theory in a way that emphasizes its connections with statistical adjustment and testing theory, used in the deformation analysis procedure in Chapter 3 . The chosen notations could make understanding more difficult for the reader that already has some familiarity with geostatistics or testing theory, but it helps to connect the two methods. The mathematical details of the designed procedure are placed in appendices to Chapter 3, which makes this chapter better readable to readers that do not seek a full understanding of adjustment and testing theory, while all details are available. We provide additional support in the understanding of the used conventions by providing a comprehensive overview of used notations at the end of the dissertation.

The questions Q3 and Q4 require application of the designed method. We apply this method to several regions of the BNLCS, and study the resulting parameter values and their uncertainties. We focus on the relation of dynamics with morphology first (Q3), and on the geographical distribution of the parameter estimates over the BNLCS thereafter (Q4). This happens in Chapters 4 and 5 , respectively. Chapter 4 presents the results of one area in full detail. The results of areas in four other regions of the Belgian and Netherlands Continental Shelf follow in Chapter 5 in a more concise way, which allows for a discussion on the variation of sea floor dynamics over the Southern North Sea.

Morphological change parameters by themselves are not yet useful to improve a resurvey policy. Instead, the parameters need to lead to a small set of indicators that allows to prioritize the analyzed areas with respect to each other (Q5). Therefore, Chapter 6 presents a second procedure, which interprets the parameters and their uncertainties. Chapter 6 has both a methodical part and a results part. The design of a method to order the analyzed regions with respect to the size of the estimated morphodynamics and the size of potentially missed morphodynamics depends on the specific properties of the estimates. At the end of this chapter, the recommended priorities are presented in a way that allows for direct application to the resurvey policy of the Royal Netherlands Navy.

The final Chapter 7 summarizes the answers to the five subquestions, formulates an answer to the research question, and thereby concludes the current research project. Recommendations are made for further scientific research and further implementation at the RNLN, based on these answers. 


\title{
Chapter 2
}

\section{Bathymetric applications of Geostatistics}

\begin{abstract}
A statistical way to describe a surface in space is by specifying the expected value and variance at each point, and their mutual covariances. The surface is assumed to consist of a trend function and a residual function. The covariances between those points are expressed in a covariance function, based on the residual function. An application of the covariance function is Kriging, an interpolation procedure that propagates the variances and covariances of the data points to variances of the interpolated values. This approach is used widely for spatial analyses, like the interpolation of a bathymetric data set.
\end{abstract}

\section{$2.1 \quad$ Introduction}

The application of deformation analysis to a series of bathymetric surveys requires depth values and their associated uncertainties at constant positions, preferably on a regular grid. However, bathymetric surveys provide for depth values at positions that change with every survey and that are subject to positioning inaccuracies. Converting bathymetric survey positions to a regular grid is commonly done using geostatistics. Geostatistics enables the interpolation of the data to continuous depth and uncertainty surfaces. The uncertainty surface includes the inaccuracy of the interpolation. In this chapter, a geostatistical procedure is designed that works on surveys of a sea floor with potentially a slope and a sand wave pattern, and that includes the vertical and horizontal measurement uncertainties. The existence and values of the additional interpolation variances and covariances of this procedure are studied, to incorporate the interpolation errors into the error budget of deformation analysis.

The central idea of geostatistics is that a depth surface can be assumed as a superposition of a deterministic trend ${ }^{\star}$, and the remaining residuals, as shown in Section 2.2. The residuals are described by the covariance function, in Section 2.3. The covariance function is used to define the Kriging equations, in Section 2.4. In Section 2.5, we draw some conclusions. This chapter gives a

\footnotetext{
* In this chapter, the word 'trend' is used as is common in geostatistics, meaning the general spatial tendency. In the following chapters, it is used as is common in deformation analysis, meaning the general temporal tendency.
} 
background for the following chapters. The theory presented is based on Davis [2002], Chilès and Delfiner [1999], Isaaks and Srivastava [1989], and Chatfield [1989].

\subsection{Depth as a trend and its residuals}

The trend is described by a series of basis functions, see Subsection 2.2.1, and the residuals by a covariance function, see Subsection 2.2.2. Such a description using a covariance function assumes stationarity, which is treated in Subsection 2.2.3.

\subsubsection{The trend described by basis functions}

Consider a two-dimensional horizontal coordinate system in which the position of a point is defined by its coordinates $\mathbf{x}_{p}=\left(x_{p}, y_{p}\right)$, and two positions differ by $\mathbf{h}_{p q}=\left(\Delta x_{p q}, \Delta y_{p q}\right)=\left(x_{q}-x_{p}, y_{q}-y_{p}\right)$. Their distance is $h_{p q}=\left\|\mathbf{h}_{p q}\right\|$. We represent the depth of the sea floor $d(\mathbf{x})$ by a superposition of a spatial trend function $m(\mathbf{x})$ and a function describing the remaining residuals $r^{(\mathrm{m})}(\mathbf{x})$ :

$$
d(\mathbf{x})=m(\mathbf{x})+r^{(\mathrm{m})}(\mathbf{x}) .
$$

Figure 2.1 shows this situation. The trend function $m(\mathbf{x})$ is deterministic, i.e. it does not include random processes. We will also call this the morphological characterization. It is expressed as a linear combination of basis functions $a_{k}(\mathbf{x})$, using coefficients $u_{k}$ :

$$
m(\mathbf{x})=\sum_{k=0}^{K-1} u_{k} a_{k}(\mathbf{x})
$$

The first function is commonly $a_{0}(\mathbf{x})=1$, to account for the depth at the origin $\mathbf{x}=(0,0)$. In case of an approximately linearly sloping sea floor, the basis function for $k=1$ and $k=2$ are $a_{1}(\mathbf{x})=x$ and $a_{2}(\mathbf{x})=y$. The basis functions are chosen such that its expected value $\mathrm{E}\{d(\mathbf{x})\}$ equals $m(\mathbf{x})$, and $\mathrm{E}\left\{r^{(\mathrm{m})}(\mathbf{x})\right\}=0$. In this chapter we limit the number of basis functions to $K=3$.

\subsubsection{The residuals described by covariances}

\section{A posteriori approach}

The residual function $r^{(\mathrm{m})}(\mathbf{x})$ describes the morphological dispersion, i.e. sea floor structure. This is considered to be a random function with an expected value of zero. A random function is a function describing a random process. It is used to define the covariance between points $\mathbf{x}_{p}$ and $\mathbf{x}_{q}$ as

$$
c^{(\mathrm{m})}\left(\mathbf{x}_{p}, \mathbf{x}_{q}\right)=\mathrm{E}\left\{r^{(\mathrm{m})}\left(\mathbf{x}_{p}\right) r^{(\mathrm{m})}\left(\mathbf{x}_{q}\right)\right\} .
$$

The covariance between two values of the same function is also called autocovariance. The autocovariance at distance $h=0$ equals the variance $c^{(\mathrm{m})}\left(\mathbf{x}_{p}, \mathbf{x}_{p}\right)$, 

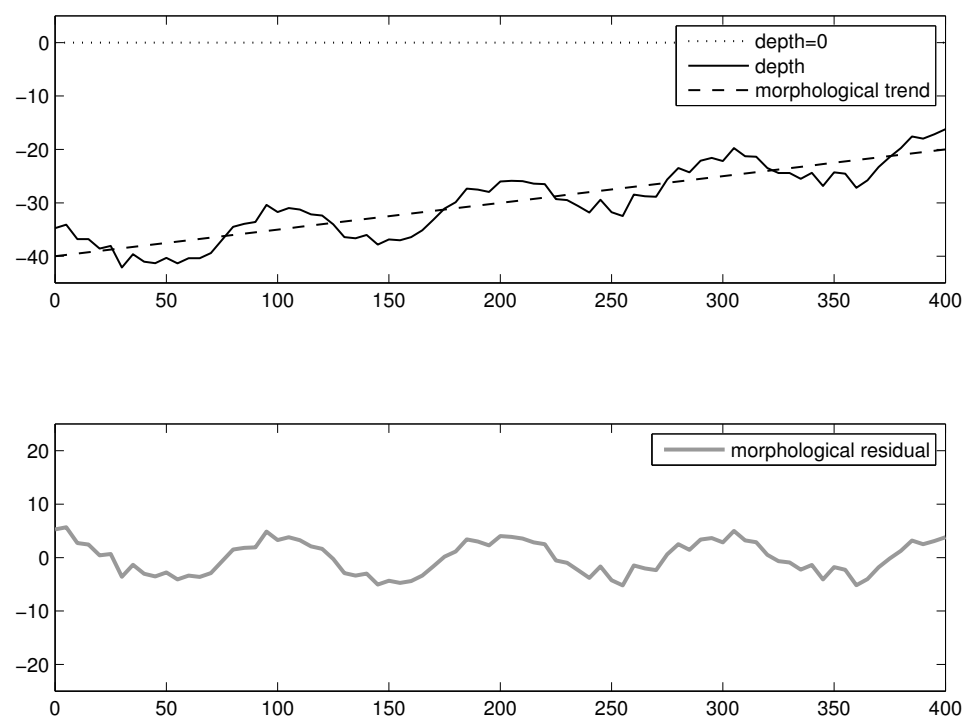

Figure 2.1: Sketch of depth $d(\mathbf{x})$ (solid line) under sea level (dotted line), morphological characterization $m(\mathbf{x})$ (dashed line), and their difference: the morphological residual $r^{(\mathrm{m})}(\mathbf{x})$ (gray line).

or $\sigma^{(\mathrm{m}) 2}\left(\mathbf{x}_{p}\right)$. It is often useful to collect all variances and covariances in a covariance matrix $\mathbf{C}_{p q}^{(\mathrm{m})}$, that has the variances on its main diagonal, while the offdiagonal elements give the covariance. Because $c^{(\mathrm{m})}\left(\mathbf{x}_{p}, \mathbf{x}_{q}\right)$ equals $c^{(\mathrm{m})}\left(\mathbf{x}_{q}, \mathbf{x}_{p}\right)$, this matrix is symmetric with respect to the main diagonal.

Using equation (2.3), we cannot calculate the covariance matrix before the residual function $r(\mathbf{x})$ is available. Such an approach is called a posteriori, because the calculation of the covariance is done after the calculation of the residuals.

\section{A priori approach}

If we have prior statistical knowledge about the structure of the residual function $r(\mathbf{x})$, an a priori approach is also possible. If $r(\mathbf{x})$ consists of $I$ uncorrelated components $r_{i}(\mathbf{x})$ with known covariance functions $c_{i}\left(\mathbf{x}_{p}, \mathbf{x}_{q}\right)$, the application of equation (2.3) is not necessary. The advantage of this is that it is not necessary to know $r(\mathbf{x})$ before calculating $c\left(\mathbf{x}_{p}, \mathbf{x}_{q}\right)$. The relation of the covariance functions is called the propagation of variances, and is given by e.g. Koch [1999] and Teunissen [2000]. 
Let us assume the uncorrelated components $r_{i}(\mathbf{x})$ have a linear relation to $r(\mathbf{x})$ via the coefficients $a_{i}$ :

$$
r(\mathbf{x})=\sum_{i=1}^{I} a_{i} r_{i}(\mathbf{x}) .
$$

The matrix $\mathbf{C}_{i, p q}$ contains the autocovariances $c_{i}\left(\mathbf{x}_{p}, \mathbf{x}_{q}\right)$. The covariances are propagated as:

$$
\mathbf{C}_{p q}=\sum_{i=1}^{I} a_{i}^{2} \mathbf{C}_{i, p q}
$$

or, equivalently:

$$
c\left(\mathbf{x}_{p}, \mathbf{x}_{q}\right)=\sum_{i=1}^{I} a_{i}^{2} c_{i}\left(\mathbf{x}_{p}, \mathbf{x}_{q}\right)
$$

\subsubsection{Stationarity}

A function $f(\mathbf{x})$ is first order stationary, if its expected value $\mathrm{E}\{f(\mathbf{x})\}$ is independent of the location $\mathbf{x}$. It is second order stationary, if it is first order stationary and the covariance $c$ between two positions only depends on position difference $\mathbf{h}$, and not on position itself. This means $c=c(\mathbf{h})$, for all $\mathbf{h}$ including the variance $c(0)$. The residual function $r(\mathbf{x})$ of such a covariance function $c(\mathbf{h})$ is said to describe uniform morphology.

\subsection{Covariance functions}

The residual function $r(\mathbf{x})$, introduced in Section 2.2, is random, and therefore cannot be described by a combination of deterministic functions. However, there is another way to describe it statistically: by its covariance function. First, we introduce the covariance function in general, and study its properties, in Subsection 2.3.1. Then, we study the effects of measurement errors in Subsection 2.3.2, and the benefits of the assumption that the covariance is independent of direction, known as isotropy, in Subsection 2.3.3. We continue in subsection 2.3.4 with the application of the theory to a discrete set of depth values, which we apply to bathymetry in Subsection 2.3.5.

\subsubsection{Definition and properties of covariance functions}

The covariance function $c(\mathbf{h})$ of the second order stationary random function of residuals $r(\mathbf{x})$ is defined as

$$
c(\mathbf{h})=\mathrm{E}\{r(\mathbf{x}) r(\mathbf{x}+\mathbf{h})\} .
$$

Covariance functions are positive definite [Chilès and Delfiner, 1999], meaning that all variance calculations lead to nonnegative results. The covariance function is also symmetric: $c(\mathbf{h})=c(-\mathbf{h})$. Normalization of autocovariance results in autocorrelation $c(\mathbf{h}) / c(0)$. 


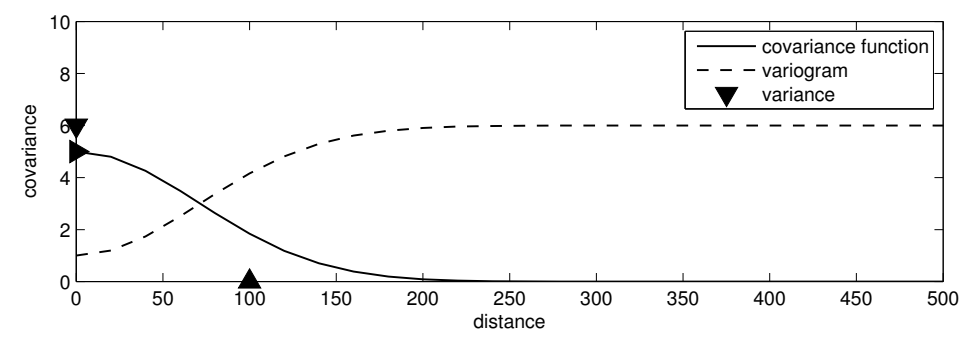

Figure 2.2: Sketches of Gaussian covariance functions. Solid line: a one-dimensional covariance function; dashed line: the corresponding variogram. Visible are the sill (down-pointing triangle, and asymptotic value of the variogram), the nugget (difference between down-pointing triangle and right-pointing triangle, and value of the variogram at zero distance) and the inflection point (up-pointing triangle). For the Gaussian function, the range is about twice the inflection point.

An alternative way of expressing the similarity between two points at distance $h$ is the variogram $\gamma(\mathbf{h})$ [Chilès and Delfiner, 1999],

$$
\gamma(\mathbf{h})=c(0)-c(\mathbf{h})(\text { if } h>0) .
$$

The value $\gamma(0)$ is called the nugget, which accounts for spatially uncorrelated variations. The covariance function $c(\mathbf{h})$ has a discontinuity at $h=0$ of the size of the nugget. This discontinuity is known as the nugget effect. Its value $c(0)$ is the sill. The correlated part $c(0)-\gamma(0)$ is denoted $c_{0}$.

The covariance function approximates zero for large distances. The distance beyond which no covariance is assumed to exist is the range $h_{\max }: c\left(h>h_{\max }\right)=$ 0 . An example of a one-dimensional covariance function and the corresponding variogram are shown in Figure 2.2.

\subsubsection{Influences of measurement errors on the covariance function}

\section{Measured depth values}

If depth $d(\mathbf{x})$ is measured, the morphological residuals $r^{(\mathrm{m})}(\mathbf{x})$ are subject to the stochastic influence $\underline{e}^{(\mathrm{v})}$ that is associated with each measurement. Their combined random function is denoted $\underline{r}^{(\mathrm{mv})}(\mathbf{x})$ :

$$
\underline{r}^{(\mathrm{mv})}(\mathbf{x})=r^{(\mathrm{m})}(\mathbf{x})+\underline{e}^{(\mathrm{v})}(\mathbf{x}) .
$$

To indicate stochastic parameters, we underline them. Superscript $\mathrm{m}$ indicates the influence of morphological variation, and superscript $\mathrm{v}$ the influence of depth measurement. Figure 2.3 shows this situation. It is assumed that these depth measurement errors $\underline{e}^{(\mathrm{v})}$ have a normal distribution $\mathcal{N}\left(0, \sigma^{(\mathrm{v}) 2}\right)$, with their own covariance function $c^{(\mathrm{v})}(\mathbf{h})$. The uncorrelated part of $\sigma^{(\mathrm{v}) 2}$ forms the nugget effect $\gamma^{(\mathrm{v})}(0)$. 


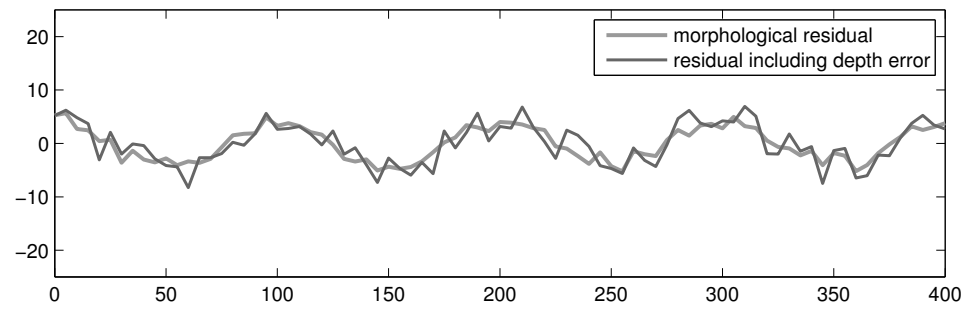

Figure 2.3: Sketch of residual $\underline{r}^{(\mathrm{mv})}(\mathbf{x})$ (thin line) and residual $r^{(\mathrm{m})}(\mathbf{x})$ (thick line).

The covariance function of the morphological residuals $r(\mathbf{x})$ is denoted $c^{(\mathrm{m})}(\mathbf{x})$. We assume that the measurement process is independent from the morphology, and we can therefore propagate the covariances according to equation (2.6):

$$
c^{(\mathrm{mv})}(\mathbf{h})=c^{(\mathrm{m})}(\mathbf{h})+c^{(\mathrm{v})}(\mathbf{h}) .
$$

\section{Measured positions}

If positions $\mathbf{x}_{p}$ are measured as well, we must account for the contribution of positioning errors $\underline{\mathbf{e}}^{(1)}$ to the depth error, as the depth values are not located correctly. Measured positions

$$
\underline{\mathbf{x}}_{p}^{(\mathrm{l})}=\mathbf{x}_{p}+\underline{\mathbf{e}}_{p}^{(\mathrm{l})}
$$

are available, instead of the true positions $\mathbf{x}_{p}$. This also affects the morphological residuals $\underline{r}^{(\mathrm{mv})}(\mathbf{x})$. We denote the combined effect of the horizontal and vertical error as $\underline{e}^{(\mathbf{h v})}(\mathbf{x})$. This combined error changes the residual function to

$$
\underline{r}^{(\mathrm{hmv})}(\mathbf{x})=r^{(\mathrm{m})}(\mathbf{x})+\underline{e}^{(\mathrm{hv})}(\mathbf{x}),
$$

see also Figure 2.4. Therefore, the covariance function changes as well:

$$
c^{(\mathrm{hmv})}(\mathbf{h})=c^{(\mathrm{m})}(\mathbf{h})+c^{(\mathrm{hv})}(\mathbf{h}) .
$$

We derive the covariances $c^{(\mathrm{hmv})}(\mathbf{h})$ from the measured differences $\underline{\mathbf{h}}_{p q}$ between positions $\underline{\mathbf{x}}_{p}$ and $\underline{\mathbf{x}}_{q}$ :

$$
\underline{\mathbf{h}}_{p q}^{(\mathrm{l})}=\mathbf{h}+\underline{\mathbf{e}}_{p}^{(1)}-\underline{\mathbf{e}}_{q}^{(1)}
$$

This situation has been described by Chilès [1976], Gabrosek and Cressie [2002], and Cressie and Kornak [2003]. The probability density function $p\left(\underline{\mathbf{e}}^{(1)}\right)$ for the error in position measurement is defined in the two-dimensional field of real numbers $\mathbb{R}^{2}$. Usually, it is assumed to follow an unbiased Gaussian function $\mathcal{N}\left(0, \sigma^{(1) 2}\right)$. First, we make the assumption that position measurements are uncorrelated. The relation between the covariance functions $c^{(\mathrm{mv})}(\mathbf{h})$ and $c^{(\mathrm{hmv})}(\mathbf{h})$ is in that case [Chilès, 1976]:

$$
c^{(\mathrm{hmv})}(\mathbf{h})=\iint_{\mathbb{R}^{2}} \iint_{\mathbb{R}^{2}} c^{(\mathrm{mv})}(\mathbf{h}) \mathrm{p}\left(\underline{\mathbf{e}}_{p}^{(\mathrm{l})}\right) \mathrm{p}\left(\underline{\mathbf{e}}_{q}^{(\mathrm{l})}\right) \mathrm{d} \underline{\mathbf{e}}_{q}^{(\mathrm{l})} \mathrm{d} \underline{\mathbf{e}}_{p}^{(\mathrm{l})} .
$$




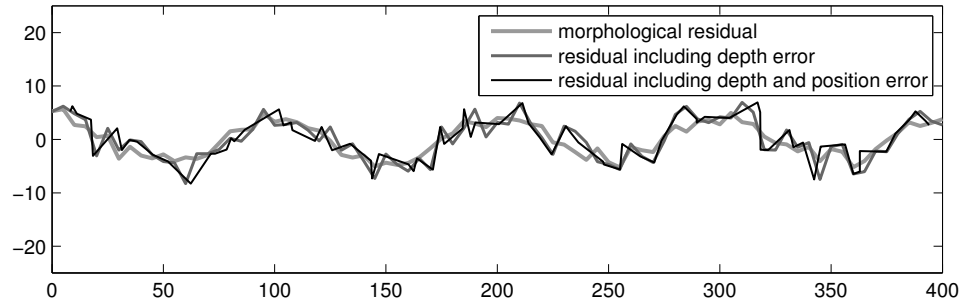

Figure 2.4: Sketch of residual $\underline{r}^{(\mathrm{hmv})}(\mathbf{x})$ (thin line), residual $r^{(\mathrm{mv})}(\mathbf{x})$ (medium line), and residual $r^{(\mathrm{m})}(\mathbf{x})$ (thick line).

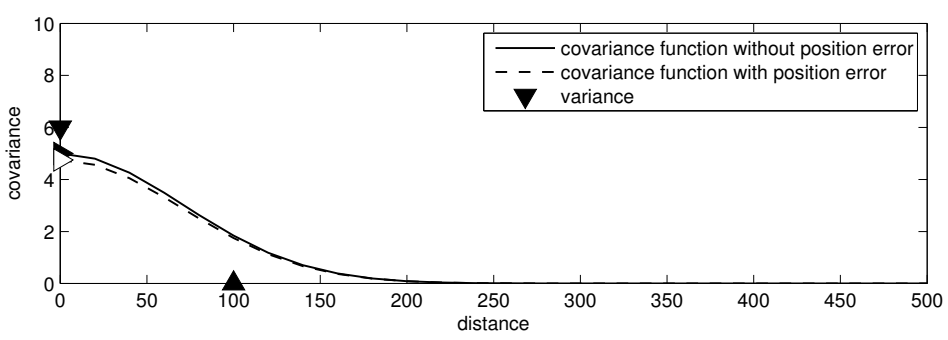

Figure 2.5: Sketch of the effect of position errors on the covariance function, after Chilès [1976]. A one-dimensional covariance function $c^{(\mathrm{mv})}(\mathbf{h})$ without the influence of position measurement (solid line) and the corresponding covariance function $c^{(\mathrm{hmv})}(\mathbf{h})$ with the influence of position measurement (dashed line), in this case Gaussian functions.

As illustrated in Figures 2.4 and 2.5, the introduction of positioning inaccuracies decreases the correlation at small distances $h \rightarrow 0$, and thus results in a larger nugget effect. Equation (2.14) shows that $\underline{\mathbf{e}}_{p}^{(1)}-\underline{\mathbf{e}}_{q}^{(1)}$ will, on average, increase the distance $\underline{h}_{p q}^{(1)}=\left\|\underline{\mathbf{h}}_{p q}^{(1)}\right\|$ for these distances:

$$
\lim _{h_{p q} \rightarrow 0} E\left\{\underline{h}_{p q}^{(\mathrm{l})}\right\}=E\left\{\left\|\underline{\mathbf{e}}_{p}^{(\mathrm{l})}-\underline{\mathbf{e}}_{q}^{(\mathrm{l})}\right\|\right\}>0
$$

At larger distances, $E\left\{\underline{\mathbf{h}}_{p q}^{(1)}\right\}$ approximates $E\left\{\mathbf{h}_{p q}\right\}$, because here $E\left\{\left\|\underline{\mathbf{e}}_{p}^{(1)}-\underline{\mathbf{e}}_{q}^{(1)}\right\|\right\}$ will approximate zero, and the difference between the two covariance functions disappears.

Usually, measured positions are positively correlated at small time differences, which correspond to small position differences in track direction. See e.g. Amiri-Simkooei and Tiberius [2006] for correlation of satellite navigation system positions in time. Compared to the uncorrelated situation, the expected positioning error for small distances decreases, and as a consequence the difference between $E\left\{h_{p q}\right\}$ and $E\left\{\underline{h}_{p q}^{(1)}\right\}$ decreases too. Therefore, $c^{(\mathrm{hmv})}(\mathbf{h})$ becomes more similar to $c^{(\mathrm{mv})}(\mathbf{h})$. 


\section{Binned positions}

The original data might be binned, which means a single value is assumed to represent the value everywhere in a rectangular bin, or grid cell, of a certain size. The value, measured somewhere in the bin, is thereby shifted to its center. Cressie and Kornak [2003] and Gabrosek and Cressie [2002] show that the effect of binning on the variogram is similar to that of horizontal positioning inaccuracies, as given by equation (2.15).

\subsubsection{The effect of isotropy on the covariance function}

Instead of the Cartesian coordinate differences $(\Delta x, \Delta y)$, we could also use polar coordinates $(h, \theta)$ to express the vector of position differences $\mathbf{h}$. Azimuth $\theta$ is East of North, $\theta=\tan (\Delta x / \Delta y)$, and $(\Delta x, \Delta y)=(h \sin \theta, h \cos \theta)$. If a covariance function $c(\mathbf{h})$ depends on the azimuth $\theta$, it is called anisotropic. Two special situations are specified for two-dimensional covariance functions:

1. anisotropy in scale: the covariance is expressed as

$$
c(\mathbf{h})=c\left(h, \theta^{(\mathrm{x})}, s\right),
$$

in which $s$ is a horizontal scale factor, $s>1$, relating the direction of highest variability $\theta^{(\mathrm{x})}$ to the perpendicular direction of lowest variability $\theta^{(\mathrm{y})}$;

2. isotropy: the covariance is direction-independent, $s=1$, and thus described by

$$
c(\mathbf{h})=c(h) .
$$

Examples are given in Figure 2.6. If the anisotropy is not an anisotropy in scale, the covariance function might not be positive-definite anymore, and should be approximated by such a scale anisotropy, see also Christakos [1984].

To describe scale anisotropy, we consider a second two-dimensional coordinate system in which a position is defined by its coordinates $\mathbf{x}_{p}^{(\mathrm{ps})}=\left(x_{p}^{(\mathrm{ps})}, y_{p}^{(\mathrm{ps})}\right)$, and two positions differ by $\mathbf{h}_{p q}^{(\mathrm{ps})}=\left(\Delta x_{p q}^{(\mathrm{ps})}, \Delta y_{p q}^{(\mathrm{ps})}\right)$. The $x^{(\mathrm{ps})}$-axis of the new system coincides with the direction $\theta^{(\mathrm{x})}$, and the $y^{(\mathrm{ps})}$-axis coincides with a direction $90^{\circ}$ counter-clockwise. Those two axes coincide with the principal directions of the covariance and are scaled with factor $s$. The relation between a position in the original system $\mathbf{x}^{(\mathrm{o})}$ and the same position in the scaled system $\mathbf{x}^{(\mathrm{ps})}$ is

$$
\left[\begin{array}{l}
x^{(\mathrm{ps})} \\
y^{(\mathrm{ps})}
\end{array}\right]=\left[\begin{array}{cc}
\cos \theta^{(\mathrm{x})} & -\sin \theta^{(\mathrm{x})} \\
s \sin \theta^{(\mathrm{x})} & s \cos \theta^{(\mathrm{x})}
\end{array}\right]\left[\begin{array}{c}
x^{(\mathrm{o})} \\
y^{(\mathrm{o})}
\end{array}\right] .
$$

Consecutively, we define the scaled distance as:

$$
h^{(\mathrm{ps})}=\sqrt{\Delta x^{(\mathrm{ps}) 2}+\Delta y^{(\mathrm{ps}) 2}},
$$

enabling us to express the covariance function as:

$$
c(\mathbf{h})=c\left(h^{(\mathrm{ps})}\right) .
$$


(a) isotropic covariance function

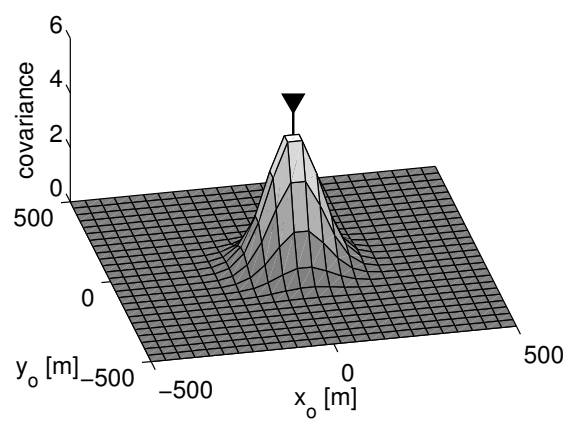

(c) isotropic covariance function with hole effect

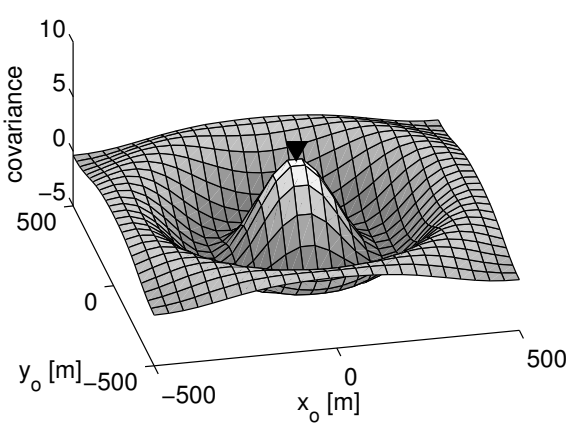

(b) anisotropic covariance function

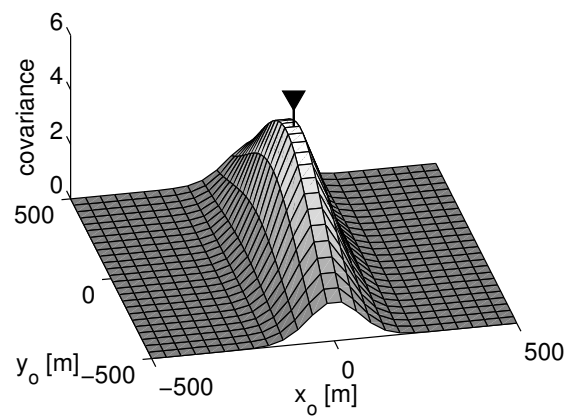

(d) anisotropic covariance function with hole effect

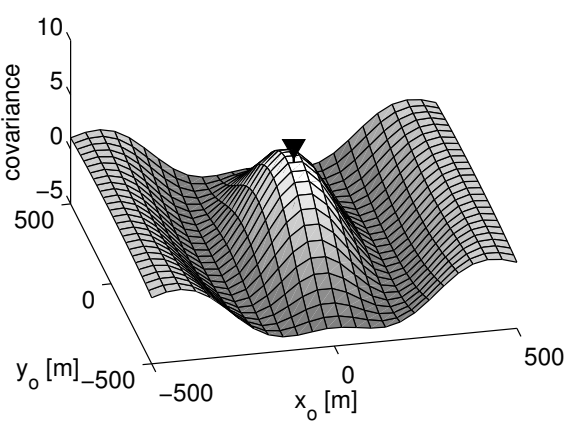

Figure 2.6: Sketches of Gaussian covariance functions in two dimensions. The sill is visible as a down-pointing triangle. The figures at the left are isotropic, and the figures at the bottom row contain a hole effect, due to some rhythmic pattern. The anisotropy of graphs (b) and (d) is anisotropy in scale.

In this new system, the covariance function with scale-anisotropy is isotropic. The covariance function is described in the original system by the sill $c(0)$, the correlated variance $c_{0}$, the direction $\theta^{(\mathrm{x})}$, and the two ranges $\Delta y_{\max }$ and $\Delta x_{\max }$. The scale factor $s$ is derived from these ranges as $\Delta y_{\max } / \Delta x_{\max }$. An example is given in Figure 2.7.

\subsubsection{The empirical covariance function}

In practice, we work with data sets of the sea floor, the consequences of which are discussed in this section. To guarantee second order stationarity, uniform morphology $d(\mathbf{x})$ per data set is necessary. Segmentation of the sea floor into areas that are a morphological unit is e.g. done by Pluymaekers [2007] and Pluymaekers et al. [2007]. We take a pragmatic approach here, and create area boundaries based on prior knowledge of the sea floor, as e.g. available in nautical charts. 


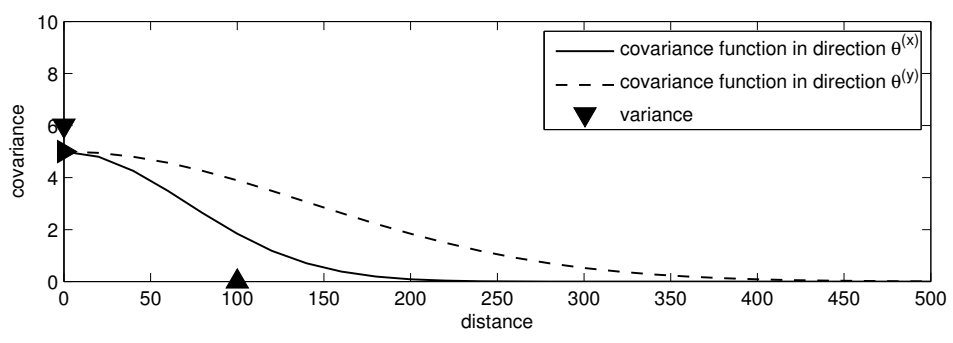

Figure 2.7: Sketches of Gaussian covariance functions, that define a two-dimension covariance function with anisotropy in scale. Solid line: direction $\theta^{(\mathrm{x})}$; dashed line: direction $\theta^{(\mathrm{y})}$.

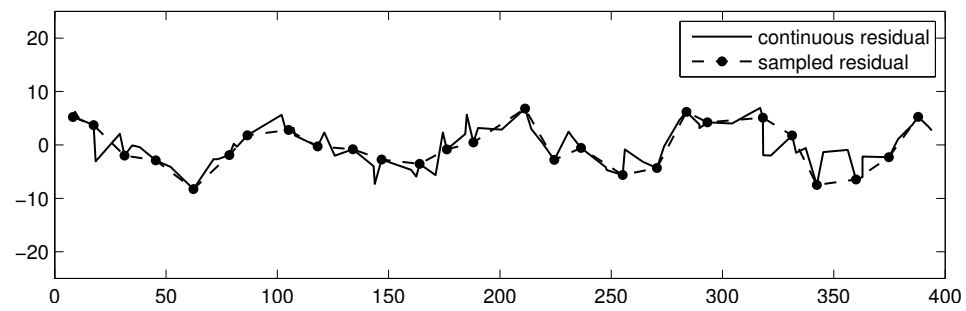

Figure 2.8: Sketch of the residual $r^{(\mathrm{hmv})}(\mathbf{x})$ (solid line), and its discrete realization $\underline{r}^{(\mathrm{hmrv})}\left[\mathbf{x}_{p}\right]$ (black dots and dashed line).

Only a discrete realization $\underline{r}^{(\mathrm{hmrv})}\left[\mathbf{x}_{p}\right]$ of the residual function $\underline{r}^{(\mathrm{hmv})}(\mathbf{x})$ is available, at the $P$ available data points $\mathbf{x}_{p}$. See Figure 2.8 for an example. The relation between the two residual functions is:

$$
\underline{r}^{(\mathrm{hmrv})}(\mathbf{x})=\underline{r}^{(\mathrm{hmv})}(\mathbf{x})+e^{(\mathrm{r})}(\mathbf{x}) .
$$

For the discrete set of data positions $\mathbf{x}_{p}$, the realization error $e^{(\mathrm{r})}\left[\mathbf{x}_{p}\right]$ equals zero, and thus the two residual functions are equal there. Although the covariance function $\underline{c}^{(\mathrm{hmrv})}(\mathbf{x})$ will be based on the discrete residual function $\underline{r}^{(\mathrm{hmrv})}\left[\mathrm{x}_{p}\right]$, it actually describes a continuous function $\underline{r}^{(\mathrm{hmrv})}(\mathbf{x})$. The continuous covariance function is only useful if the resolution of data points $\mathbf{x}_{p}$ is high enough to guarantee that $\underline{c}^{(\mathrm{hmrv})}(\mathbf{x})$ approximates their true covariance function $c^{(\mathrm{hmv})}(\mathbf{x})$. This is a necessary condition to ensure that a continuous function $\underline{r}^{(\mathrm{hmrv})}(\mathbf{x})$ is close to the real function of residuals $\underline{r}^{(\mathrm{hmv})}(\mathbf{x})$. (In the remainder of this section, the superscripted indices of the covariance and residual functions are dropped for brevity.)

Firstly, the discrete covariance function $\underline{c}[\mathbf{h}]$ is calculated by considering a number of intervals $(i, j)$, where $i$ is the index to the distance interval, and $j$ to the azimuth interval. In case of scale anisotropy, $\theta_{j}$ is one of two perpendicular 

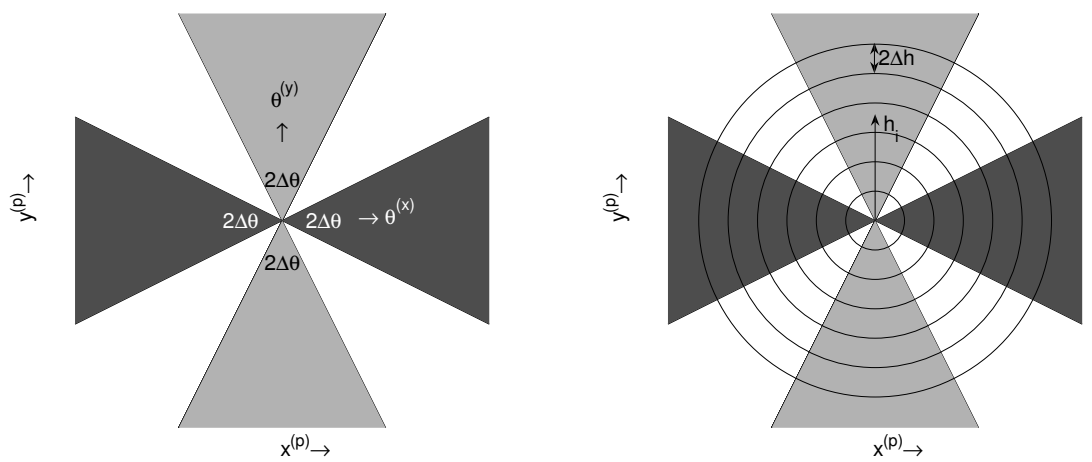

Figure 2.9: Sketches of azimuth intervals $\left[\theta_{j}-\Delta \theta, \theta_{j}+\Delta \theta\right]$ (left) and distance intervals $\left[h_{i}-\Delta h, h_{i}+\Delta h\right]$ (right) for the calculation of covariances $c_{j}\left[\bar{h}_{i}\right]$. The axes of the rotated coordinate system are indicated by $x^{(\mathrm{p})}$ and $y^{(\mathrm{p})}$.

directions $\underline{\theta}^{(\mathrm{x})}$ and $\underline{\theta}^{(\mathrm{y})}$ between $0^{\circ}$ and $180^{\circ}$. In that case, we define an interval as $\left[h_{i}-\Delta h, h_{i}+\Delta h\right]$ and $\left[\theta_{j}-\Delta \theta, \theta_{j}+\Delta \theta\right]$, with $\Delta h$ half the interval size for distance, and $\Delta \theta$ half the interval size for azimuth. The azimuth interval includes its opposing interval $\left[180^{\circ}+\theta_{j}-\Delta \theta, 180^{\circ}+\theta_{j}+\Delta \theta\right]$. This is illustrated in Figure 2.9.

Using these intervals, equation (2.7) is reformulated as [Chatfield, 1989]:

$$
\underline{c}_{j}\left[\bar{h}_{i}\right]=\sum_{p=1}^{P^{*}} \underline{r}\left[\mathbf{x}_{p}\right] \underline{r}\left[\mathbf{x}_{p}+\mathbf{h}_{i}^{*}\right] / P^{*},
$$

where $P^{*}$ is the number of values of $\underline{r}$ at position differences $\mathbf{h}_{i}^{*}$, at distances $h_{i}^{*}$ between $h_{i}-\Delta h$ and $h_{i}+\Delta h$. Further, those position differences have directions $\theta_{i}^{*}$ between $\underline{\theta}_{j}-\Delta \theta$ and $\underline{\theta}_{j}+\Delta \theta$, and $\bar{h}_{i}$ is the average distance between all pairs in the interval. Equation (2.23) can also be derived via least-squares variance component estimation, as shown by Teunissen and Amiri-Simkooei [2008].

The coordinate transformation of equation (2.19) is performed in two steps. First, we find the principal direction $\underline{\theta}^{(\mathrm{x})}$. The rotated coordinate system of which the directions of the axes coincide with the principal directions is denoted $\left(x^{(\mathrm{p})}, y^{(\mathrm{p})}\right)$. It is defined as

$$
\left[\begin{array}{l}
x^{(\mathrm{p})} \\
y^{(\mathrm{p})}
\end{array}\right]=\left[\begin{array}{cc}
\cos \underline{\theta}^{(\mathrm{x})} & -\sin \underline{\theta}^{(\mathrm{x})} \\
\sin \underline{\theta}^{(\mathrm{x})} & \cos \underline{\theta}^{(\mathrm{x})}
\end{array}\right]\left[\begin{array}{l}
x^{(\mathrm{o})} \\
y^{(\mathrm{o})}
\end{array}\right]
$$

After that, we use this system to calculate the scale parameter $\underline{s}$ via the fit of two covariance functions in the two principal directions. We are then able to work in the scaled coordinate system $\left(x^{(\mathrm{ps})}, y^{(\mathrm{ps})}\right)$ :

$$
\left[\begin{array}{c}
x^{(\mathrm{ps})} \\
y^{(\mathrm{ps})}
\end{array}\right]=\left[\begin{array}{c}
x^{(\mathrm{p})} \\
\underline{s} y^{(\mathrm{p})}
\end{array}\right] .
$$




\section{Direction of highest variability}

First, the azimuth of highest variability $\theta^{(\mathrm{x})}$ needs to be found. It is the direction of the average gradient of the data. In case a rhythmic pattern is present, the direction of the survey tracks has often been chosen accordingly. In such a case, the survey direction is close to $\theta^{(\mathrm{x})}$. Alternatively, the direction of highest variability could be estimated, e.g. by the DIGIPOL algorithm [Van Munster et al., 1995; RIKZ, 1997; De Koning, 2007]. We propose a simplified version of this algorithm [Dorst, 2004], consisting of the following four steps.

1. Grid $\underline{r}\left[\mathbf{x}_{p}\right]$ to a dense grid in coordinate system $\mathbf{x}^{(0)}$, and do not mind about empty nodes. This results in $\underline{r}\left[\mathbf{x}_{(l, m)}\right]$ in the grid of size $L \times M$;

2. Calculate the local gradient $\nabla \underline{r}\left[\mathbf{x}_{(l, m)}\right]$ by the differences around each grid node $(l, m)$ in both the directions $x$ and $y$ :

$$
\nabla \underline{r}\left[\mathbf{x}_{(l, m)}\right]=\left[\begin{array}{c}
\frac{\underline{r}\left[\mathbf{x}_{(l+1, m)}\right]-\underline{r}\left[\mathbf{x}_{(l-1, m)}\right]}{x_{(l+1, m)}-x_{(l-1, m)}} \\
\frac{\underline{r}\left[\mathbf{x}_{(l, m+1)}\right]-\underline{r}\left[\mathbf{x}_{(l, m-1)}\right]}{y_{(l, m+1)}-y_{(l, m-1)}}
\end{array}\right] .
$$

The gradient has magnitude $\left\|\nabla \underline{r}\left[\mathbf{x}_{(l, m)}\right]\right\|$, and azimuth $\underline{\theta}^{(\mathrm{g})}\left[\mathbf{x}_{(l, m)}\right]$. Do this at those points where the neighbouring nodes are not empty;

3. Project all local gradients into the direction $\theta$, for $\theta$ in $\left[\vartheta, 180^{\circ}\right]$, and change this direction with increments $\vartheta$ of for instance a degree. The variability $\underline{v}[\theta]$ is:

$$
\underline{v}[\theta]=\sum_{l=1}^{L} \sum_{m=1}^{M}\left\|\nabla \underline{r}\left[\mathbf{x}_{(l, m)}\right]\right\| \operatorname{abs}\left(\cos \left(\theta-\underline{\theta}^{(\mathrm{g})}\left[\mathbf{x}_{(l, m)}\right]\right)\right) /(L M) .
$$

The absolute value is denoted abs() here.

4. Assign the direction perpendicular to the direction that gives the smallest sum of projected gradients $\underline{v}[\theta]$ to azimuth $\underline{\theta}^{(\mathrm{x})}$. If $\underline{v}[\theta]$ is independent of $\theta, \underline{r}\left[\mathbf{x}_{p}\right]$ is isotropic. If the anisotropic minimum and maximum are not approximately $90^{\circ}$ apart, the anisotropy is not an anisotropy in scale between two perpendicular axes.

Examples of $\underline{v}[\theta]$, based on Figure 2.10, are given in Figure 2.11.

A higher or lower data density in the track direction results in biased variability towards or away from the track direction. This happens for instance with single-beam data. It is clear from Figure 2.11 that the direction of lowest variability is more pronounced than the direction of highest variability. This is our reason to estimate this direction from the variability function, instead of estimating the direction of highest variability directly.

The study of the fourth surface of Figure 2.10 explains this effect. In case of a sand wave with constant crest height, the azimuths of the gradients are constant at $\underline{\theta}^{(\mathrm{g})}$, provided the grid nodes do not coincide with the peaks or 
(a) wavelengths: $667 \& 667 \mathrm{~m}$

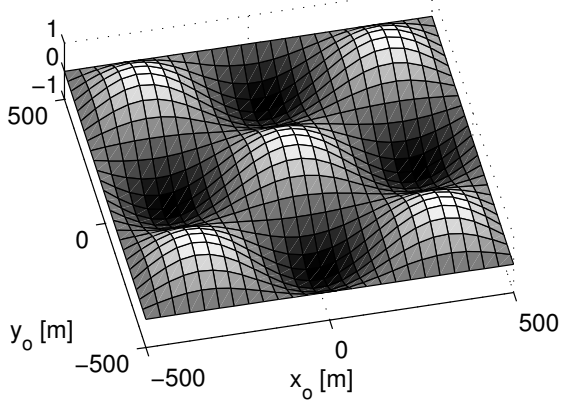

(c) wavelengths: $667 \& 1667 m$

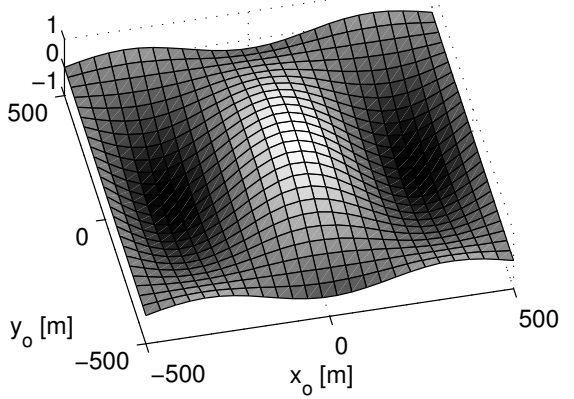

(b) wavelengths: $667 \& 1000 \mathrm{~m}$

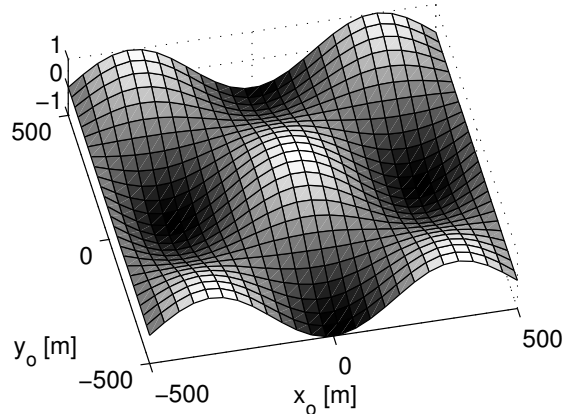

(d) wavelength: $667 \mathrm{~m}$

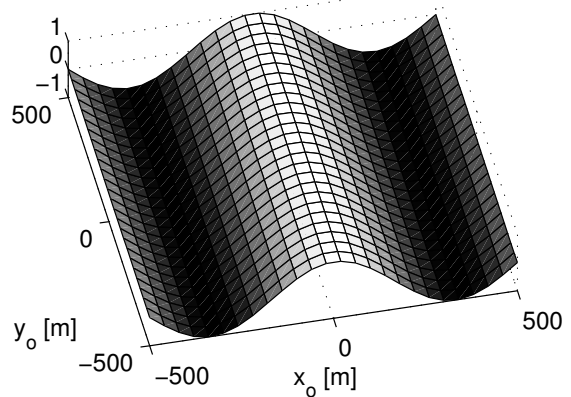

Figure 2.10: Four simulated surfaces, for which their variability $\underline{v}$ as a function of azimuth $\theta$ is shown in Figure 2.11.

troughs. In that case, equation (2.26) simplifies to express variability as the absolute of a cosine function, which makes a sharp change in slope at its zero minimum.

We define the azimuth interval of equation (2.23) as follows: $\Delta \theta=22.5^{\circ}$. Because $\theta$ is defined between zero and $180^{\circ}$, both the intervals create two areas, centered around $\underline{\theta}_{j}$ and $\underline{\theta}_{j}+180^{\circ}$. In other words, the intervals are centered around the principal directions, and together they cover half the area, see also Figure 2.9 .

The value of $\Delta \theta$ should on one hand not be too small, to provide sufficient data pairs at distances between $h-\Delta h$ and $h+\Delta h$, and on the other hand not be too wide, thereby biasing the covariance estimation for direction $\underline{\theta}_{j}$ too much. In case of single-beam surveys, the resolution in the across-track direction is very low with respect to the along-track direction. Therefore the azimuth interval cannot be very small, and $\Delta \theta=22.5^{\circ}$ gives good results.

The number of intervals for $h$ depends on the size of the area: at distances larger than half the length of its smallest cross-section, the number of pairs starts 

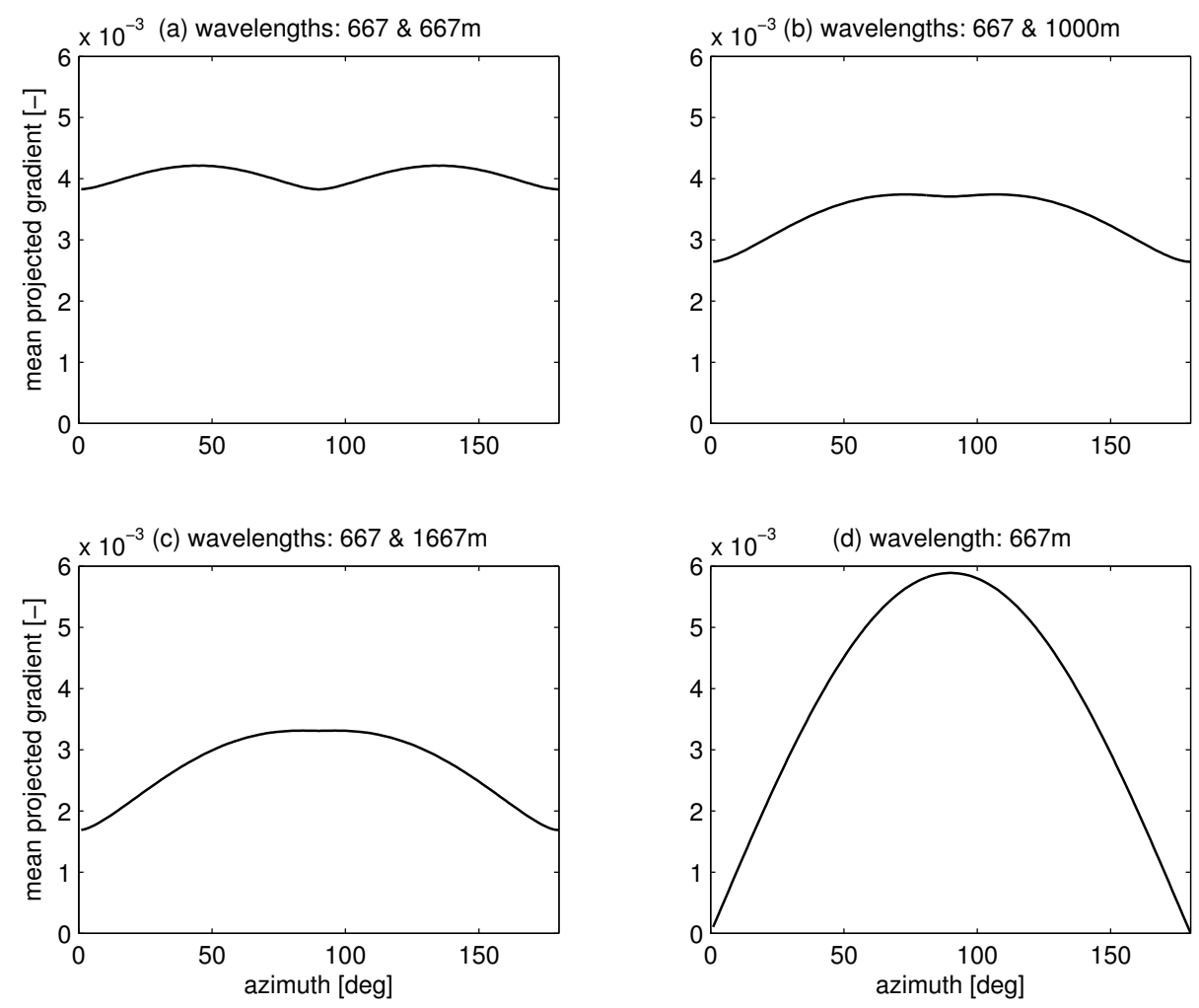

Figure 2.11: Variability $\underline{v}$ as a function of azimuth $\theta$, for the four simulated surfaces of Figure 2.10. Note that the maximum and the minimum of the function are $90^{\circ}$ apart, as long as the scales in both the directions are clearly different, and that the direction of lowest variability is more pronounced than the direction of highest variability.

to decrease, and therefore the autocovariance values become less accurate. The number of intervals is a trade-off between the number of required autocovariance values, and their accuracy. Larger intervals mean higher numbers of point pairs, and therefore a higher accuracy. However, larger intervals also mean a worse resolution of $\underline{c}_{j}[\bar{h}]$.

Several other procedures exist. Instead of subtracting neighbouring nodes, the procedure could also use a block technique in several specified directions, like demonstrated by Lindenbergh [2004]. Both Pluymaekers et al. [2007] and Calder [2006] suggest a method that uses covariance functions in various directions. Pluymaekers et al. [2007] also suggest a method per grid node, drawing profiles in various directions, and counting the number of extreme values. The wide usage and straightforward approach make the DIGIPOL algorithm the most attractive, though. 


\section{The fit of a function}

To create a continuous function, a positive definite function $\underline{c}(h)$ is fit through the calculated covariances $\underline{c}_{j}[\bar{h}]$. The function $\underline{c}(h)$ is the empirical covariance function. Christakos [1984] places restrictions on the function that could be fit. A covariance function needs to be permissible, to guarantee that this function is positive definite.

The Gaussian function is a attractive choice, because it approximates the behaviour of the covariances at small distances well, where a good fit is most important. As seen in Section 2.3.2, the effect of horizontal position uncertainty is especially prominent at small distances, resulting in a smooth behaviour at those distances, just like a Gaussian function.

The discontinuity $\underline{c}(0)$ has to be excluded from the fit. The Gaussian covariance function is

$$
c(h)=c_{0} \exp \left(-\left(h / h^{(\mathrm{i})}\right)^{2}\right),
$$

with the inflection point at $h^{(\mathrm{i})}$. In case of a rhythmic pattern, the covariance function behaves like the product of the Gaussian function and a cosine function:

$$
c(h)=c_{0} \exp \left(-\left(h / h^{(\mathrm{i})}\right)^{2}\right) \cos (\kappa h) .
$$

Here, $\kappa$ is the wave number, and $\ell=2 \pi / \kappa$ is the wavelength of the rhythmic pattern. Such a function is also called a hole effect function [Pyrcz and Deutsch, $2003]$. For distances $h$ that are small with respect to $\ell$, equation (2.29) approximates equation (2.28), see Figure 2.12. This implies that the autocovariance of a sea floor showing a 1D rhythmic pattern can be safely approximated by scalar anisotropy, for small $h$. As Figure 2.12 shows, the approximation of function (2.29) by (2.28) is valid up to the inflection point of the Gaussian function. Therefore, the covariance function for scale anisotropy should not be used at distances larger than the inflection points.

As a two-dimensional covariance function that includes a rhythmic pattern in only one dimension is not permissible, it needs to be approximated by a function that does not contain this pattern. In contrast to function (2.29), function (2.28) is always permissible, independent of the number of dimensions or the combination of the parameters of the function [Christakos, 1984]. However, Wackernagel [2003] warns against it, as the infinite differentiability at the origin may give unrealistic Kriging results.

To describe the discontinuity at $h=0$, a nugget function is necessary, which is a permissible function as well. Such a function consists of a single value to account for the nugget at $h=0$, and equals zero elsewhere. The sum of the nugget and the Gaussian function is our final covariance function, which is permissible because it is the sum of two permissible functions. The smooth Gaussian function with the addition of a nugget effect is recommended by Herzfeld [1989] for bathymetry, as the sea floor generally has a smooth nature. Wackernagel [2003] notes that the addition of a nugget effect solves the undesirable infinite differentiability of the Gaussian function. 


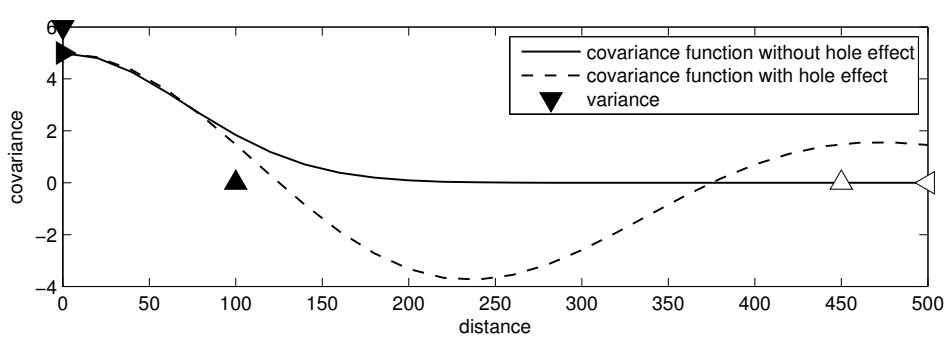

Figure 2.12: Sketch of a Gaussian covariance function with hole effect (dotted line), and its approximation by a Gaussian covariance function without hole effect (solid line). Visible are the inflection points $h^{(\mathrm{i})}$ (up-pointing triangles, open for the hole effect function, closed for the no-cosine function), and the wavelength $\ell$ of the cosine effect function (left-pointing triangle). The approximation of the hole effect function by the Gaussian function is valid at distances smaller than the inflection point of the latter.

Equation (2.23) gives us the variance $\underline{c}[0]$ as:

$$
\underline{c}[0]=\sum_{p=1}^{P} \underline{r}\left[\mathbf{x}_{p}\right]^{2} / P
$$

The parameters $\underline{c}_{0}$ and $\underline{h}^{(\mathrm{i})}$ are estimated by a fit of function $(2.28)$ on $\underline{c}_{j}[\bar{h}]$. The best fit at small distances is obtained if the covariance values of $\underline{c}_{j}[\bar{h}]$ are weighted by $P^{* 2} / \bar{h}$, so that the fit is good for small distances and reliable covariance values. If there is scale anisotropy, we estimate two inflection points $\underline{h}_{j}^{(\mathrm{i})}$, and one correlation variance parameter $\underline{c}_{0}$. The nugget $\underline{\gamma}(0)$ follows from equation (2.8). The anisotropy factor $s$ is calculated as $\underline{s}=\underline{h}^{\text {(ix) }} / \underline{h}^{\text {(iy) }}$, using the inflection points in the directions of highest and lowest variability. Another indicator of the strength of the anisotropy is the variability quotient $\max (\underline{v}[\theta]) / \min (\underline{v}[\theta])$.

An example of the fit of the two covariance functions in equation (2.28) and (2.29) is shown in Figures 2.13 and 2.14. From those figures, several observations can be made. The covariance values in direction $\underline{\theta}^{(\mathrm{y})}$ are much more spurious than those in direction $\underline{\theta}^{(\mathrm{x})}$, because of the difference in sampling density: the survey lines run approximately in the direction $\underline{\theta}^{(\mathrm{x})}$. The covariance function in direction $\underline{\theta}^{(\mathrm{x})}$ decreases faster than the one in direction $\underline{\theta}^{(\mathrm{y})}$, because the variability is higher in the direction $\underline{\theta}^{(\mathrm{x})}$. At distances larger than the inflection points, the fit gets worse, because of the limited similarity of the empirical covariance values with a Gaussian function. This is acceptable, as we will only use the covariance functions up to the inflection points. 


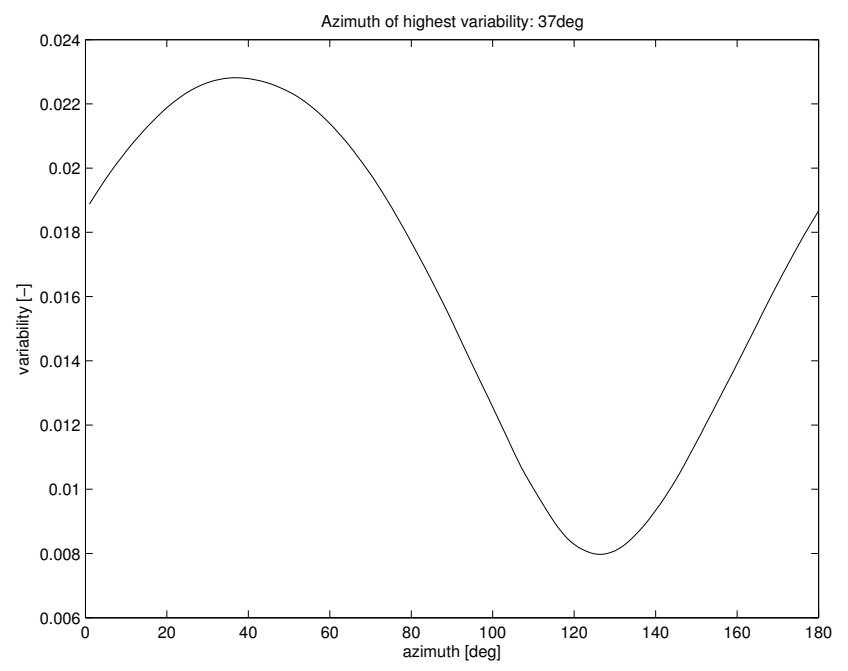

Figure 2.13: Results for the covariance function of the May 2000 survey of critical area G: variability $\underline{v}$ as a function of azimuth $\theta$. The directions $\underline{\theta}^{(\mathrm{x})}$ and $\underline{\theta}^{(\mathrm{y})}$ are $37^{\circ}$ and $127^{\circ}$ respectively, and the variability quotient is 2.8 .

\subsubsection{Usage in hydrography}

The covariance function (or the variogram) is used to describe the morphological variations of the sea floor $r(\mathbf{x})$, like the wavelength of sand waves [Robert, 1988; Robert and Richards, 1988]. In case of such rhythmic features in one dimension, it is shown above that the anisotropy can be approximated by scale anisotropy in two perpendicular principal directions.

The covariance function is also useful to express the influence of the measurement errors on the depth values. As described above, a single covariance function is able to include all correlated and uncorrelated errors, in both the horizontal and vertical directions. Autocovariance is often the highest in the direction of the ship track, and the lowest in the perpendicular direction. If the ship tracks are in the direction of highest sea floor variability, as is good practice, there are still only two principal directions in the combined covariance function, which is estimated from depth data using the above procedure. A sketch of both the covariance functions, for sea floor variation and measurement errors, and their combined total covariance function, is shown in Figure 2.15. According to the propagation law of variances, see Section 2.2.2, the combined covariance function is simply the sum of the two covariance functions. Although not strictly Gaussian, the result is still approximated by one Gaussian function in each principal direction.

The main application of the variogram and covariance function in hydrography is to estimate weights for the interpolation of depth values. Such an 


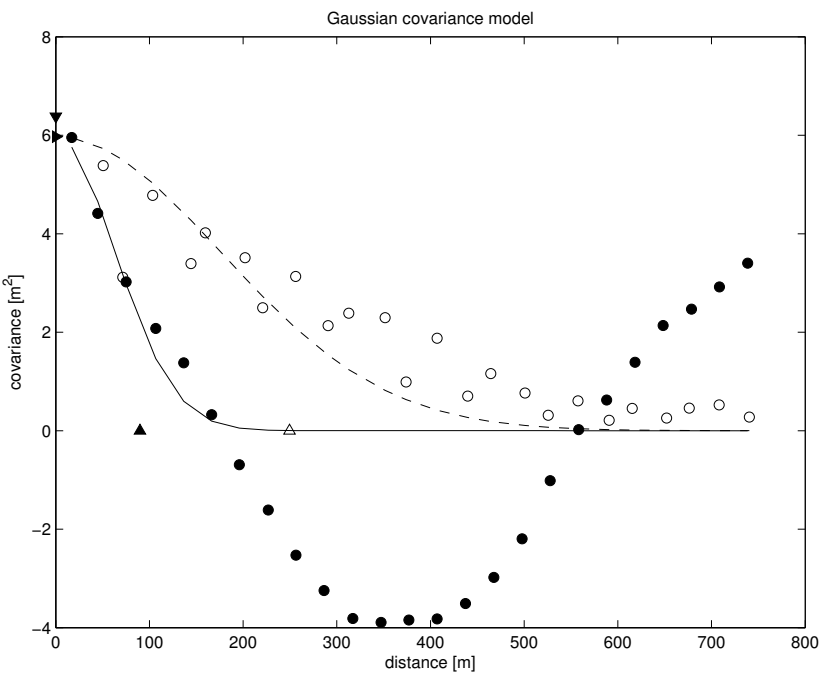

Figure 2.14: Results for the covariance function of the May 2000 survey of critical area G: the two estimated Gaussian covariance functions for the principal directions, their parameters, and the covariance values on which they are based (right). Direction $\underline{\theta}^{(\mathrm{x})}$ : solid line, closed symbols; direction $\underline{\theta}^{(\mathrm{y})}$ : dotted line, open symbols. The inflection points $\underline{h}^{(\mathrm{i})}$ (up-pointing triangles) are at $77 \mathrm{~m}$ and $136 \mathrm{~m}$, so the anisotropy factor $\underline{s}$ is 1.8. The correlated variance parameter $\underline{c}_{0}=5.83 \mathrm{~m}^{2}$ is the right pointing triangle, the sill $\underline{c}(0)=6.29 \mathrm{~m}^{2}$ is the down-pointing triangle, and the nugget effect $\underline{\gamma}(0)=0.47$ $\mathrm{m}^{2}$ their difference. The 95 -th percentile of the residuals is $1.96 \sqrt{\underline{c}(0)}=4.92 \mathrm{~m}$. The correlated part of this is $1.96 \sqrt{\underline{c}_{0}}=4.73 \mathrm{~m}$ at $95 \%$, and the uncorrelated part is the nugget effect, that has the value $1.96 \sqrt{\underline{\gamma}(0)}=1.34 \mathrm{~m}$ at $95 \%$.

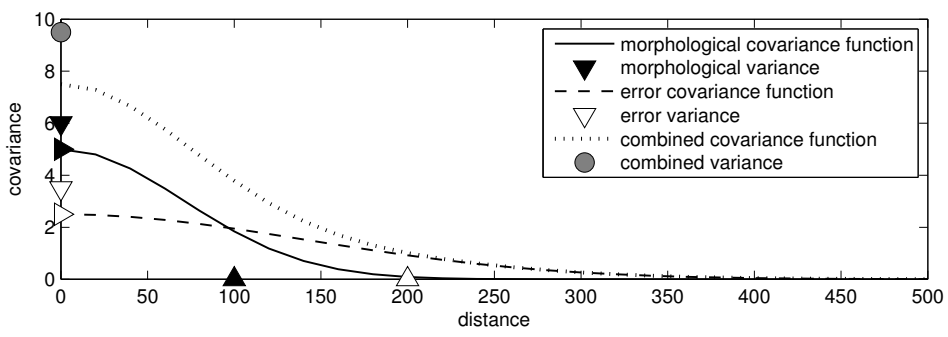

Figure 2.15: Covariance functions for the morphological variation (solid line), the measurement errors (dashed line), and their combined effect (dotted line). Visible are the inflection points $\underline{h}^{\text {(i) }}$ (up-pointing triangles, closed for the morphological covariance and open for the error covariance). The combined function is approximately Gaussian. 
estimation of the interpolation weights is called Kriging, which is the subject of the next section.

\subsection{Kriging}

The two-dimensional depth function $d(\mathbf{x})$ of the sea floor is only known by its measured values $\underline{d}[\underline{\mathbf{x}}]$, collected in a data set. The geostatistical method of Kriging is available to estimate a surface $\underline{d}(\mathbf{x})$, based on a description of the depth function as the sum of a trend function and a residual function, see Section 2.2. The residual function is described by the covariance function, as calculated in Section 2.3.

The current section describes how Kriging calculates a grid of interpolated depth values $\underline{d}[\mathbf{x}]$ and their variances $\underline{\sigma}^{2}[\mathbf{x}]$, and it also shows that the interpolated depth values are correlated in the same way as the measured depth values. First, we introduce Kriging in general, and study its properties, in Subsection 2.4.1. Then, we look at the procedure to calculate interpolation weights, in Subsection 2.4.2. Next, we look at its interpolation equations, with special emphasis on the Kriging variance, in Subsection 2.4.3. Finally, we discuss some practical implications in Subsection 2.4.4.

\subsubsection{Kriging interpolation and properties}

Interpolation methods generally calculate a value at a position $\mathbf{x}_{n}$ as a weighted average of $P_{n}$ surrounding data points $\mathbf{x}_{p}$. Let $P_{n} \times 1$ vector $\mathbf{w}$ contain the weights $w_{p}$, and $P_{n} \times 1$ vector $\underline{\mathbf{d}}_{p}$ the depth values $\underline{d}\left[\mathbf{x}_{p}\right]$. Further, let the matrix $\underline{\mathbf{C}}_{\mathbf{n}}$ describe their variances $\underline{\sigma}^{2}\left(\mathbf{x}_{p}\right)$ according to $\underline{c}^{(\mathrm{hmrv})}(0)$ on its main diagonal, and covariances $\underline{c}\left(\mathbf{x}_{p}, \mathbf{x}_{q}\right)$ according to $\underline{c}^{(\mathrm{hmrv})}(\overline{\mathbf{h}})$ as its other elements. The interpolated depth $\underline{d}_{n}=\underline{d}\left[\mathbf{x}_{n}\right]$ and its propagated variance $\sigma_{n}^{2}$ are

$$
\underline{d}_{n}=\mathbf{w}^{\mathrm{T}} \underline{\mathbf{d}}_{p}, \quad \sigma_{n}^{2}=\mathbf{w}^{\mathrm{T}} \underline{\mathbf{C}} \mathbf{w} .
$$

If interpolation is applied to the $P_{n} \times 1$ vector $\underline{\mathbf{r}}_{p}$ of residuals $\underline{r}^{(\mathrm{hmrv})}\left[\mathbf{x}_{p}\right]$ instead, we have:

$$
\underline{d}_{n}=m_{n}+\mathbf{w}^{\mathrm{T}} \underline{\mathbf{r}}_{p}, \quad \sigma_{n}^{2}=\mathbf{w}^{\mathrm{T}} \underline{\mathbf{C}} \mathbf{w} .
$$

In this equation, $m_{n}$ is the value of trend $m(\mathbf{x})$ at $\mathbf{x}_{n}$, and the product $\mathbf{w}^{\mathrm{T}} \underline{\mathbf{r}}_{p}$ is denoted $\underline{r}^{(\mathrm{n})}\left[\mathbf{x}_{n}\right]$.

Assuming second order stationarity, Kriging derives its weights from the spatial covariance function, to calculate values and their variances at grid points. The covariance function is stochastic, because it is derived from stochastic covariance values, that are in turn based on the stochastic residuals.

Kriging is a Best Linear Unbiased Estimation (BLUE) technique, meaning that the estimates have minimal variance, depend linearly on the data, and that the expected value of the estimates equals the mean. All linear interpolation methods can propagate the variances and covariances of the data to the interpolated value, but Kriging also uses the variances and covariances to estimate the weights, thereby ensuring all BLUE criteria are met. 
The covariance $\underline{c}\left(\mathbf{x}_{p}, \mathbf{x}_{q}\right)$ is denoted $\underline{c}_{p q}$ from here on, and the corresponding notation of the variance $\underline{\sigma}_{p}^{2}$ is $\underline{c}_{p p}$. If a grid is used for the interpolation, the grid points are called nodes. Kriging relates the covariance $\underline{c}_{p n}$ between a node at $\mathbf{x}_{n}$ and an available data point at $\mathbf{x}_{p}$ to the covariances $\underline{c}_{p q}$ between the data points at $\mathbf{x}_{p}$ and $\mathbf{x}_{q}$.

The positions of the nodes are not stochastic, but Kriging assumes that the covariance function between two measured positions $\underline{c}^{(\mathrm{hmrv})}(\mathbf{h})$ is equal to the covariance function between a measured position and a node, or the covariance function between two nodes, except for the variances:

$$
\underline{c}_{p q}(\mathbf{h})=\underline{c}_{p n}(\mathbf{h})=\underline{c}_{m n}(\mathbf{h}), \quad \forall h>0 .
$$

This is a consequence of the second-order stationarity assumption of Kriging.

\section{Types of Kriging}

There are three main types of Kriging, based on the number of basis functions $K$ of equation (equl):

1. simple Kriging, if the trend function is zero: $K=0$;

2. ordinary Kriging, if the trend function, denoted $m^{(\mathrm{c})}(\mathbf{x})$, is constant: $K=1$;

3. universal Kriging, if the trend function, denoted $m^{(\mathrm{u})}(\mathbf{x})$, is variable: $K>1$.

Simple Kriging estimates the interpolation weights only, from a set of covariances. Ordinary Kriging requires the introduction of a single Lagrange parameter $u_{0}^{(\mathrm{c})}$ to solve for the unknown mean, while universal Kriging uses several Lagrange parameters $u_{k}^{(\mathrm{u})}$ to describe the unknown trend.

We further distinguish between point Kriging and block Kriging. Block Kriging is used if the node is a representation of the surrounding area, which is called the support of the node. If the node is not assumed to represent a surrounding block, point Kriging is used. In our procedure, we use point Kriging, as the support of the data points, formed by the bins, was already taken into account as a source of horizontal error when the covariance function was estimated.

Therefore, we apply universal point Kriging to the residuals of the depth function $\underline{d}[\mathbf{x}]$. We do not use block Kriging, because we assume the interpolated values are only valid at the node $\mathbf{x}_{n}$ where they are calculated. We use Universal Kriging, because the residuals possibly still contain a smaller-scale spatial trend, namely a sand wave pattern.

As Armstrong [1984] points out, a difficulty of universal Kriging is the necessity to subtract the trend function from the measured surface to obtain the residuals, from which the covariance function is calculated. However, this trend function is not calculated until the universal Kriging equations are solved. Herzfeld [1989] deals with this problem for bathymetric data. We take another approach, as we limit the geographic extent of the data points used for the interpolation 


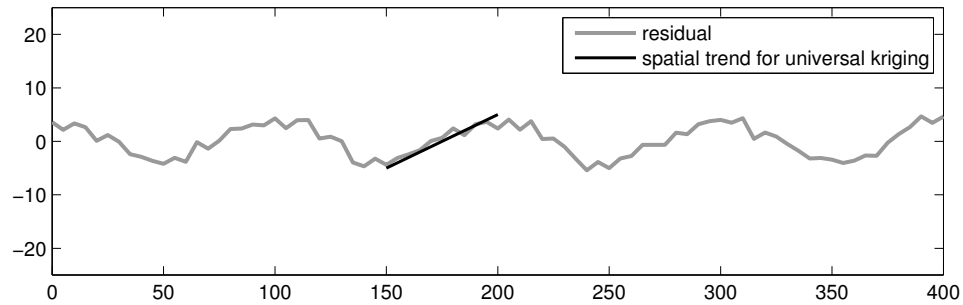

Figure 2.16: Sketch of residual function $r^{(\mathrm{hmrv})}(\mathbf{x})$ (grey line), and a local trend function $m^{(\mathrm{u})}(\mathbf{x})$ used for Universal Kriging (black line), in case of a sand wave pattern.

of a node, see below. In case of a sand wave pattern, the anisotropy in the covariance function allows for a smaller geographic extent in the pattern direction $\theta^{(\mathrm{x})}$ than in the crest direction $\theta^{(\mathrm{y})}$. Therefore, we locally approximate the sand wave by a sloping plane, which corresponds again to the choice of $K=3$, as in Section 2.2. To distinguish this Universal Kriging trend in the residuals from the overall trend $m(\mathbf{x})$ in the depth data, we denote the trend of Universal Kriging as $m^{(\mathrm{u})}(\mathbf{x})$. The situation is shown in Figure 2.16.

\subsubsection{The calculation of weights}

As concluded in Section 2.3.4, covariances between a data point at $\mathbf{x}_{p}$ and a node at $\mathbf{x}_{n}$ are assumed zero outside a circle with radius $h^{(i)}$, or, in case of scale anisotropy, outside an ellipse with semi-major axis $h^{(\mathrm{iy})}$ and semi-minor axis $h^{(\mathrm{ix})}$. Because we set covariances $\underline{c}_{p n}$ for larger distances to zero, the number of points involved is reduced from $P$ to $P_{n}$. This reduction speeds up the calculation of the weights considerably.

For each point $\mathbf{x}_{n}$, a set of equations is formulated, describing its covariance with each data point. The Kriging weights are calculated from this set of equations. These equations follow from the minimum variance constraint. To ensure unbiasedness, an additional equation for each basis function $a_{k}(\mathbf{x})$ is added to this set of equations. The basis functions have been defined by equation (2.2). A formal derivation of the universal Kriging equations can be found in Chilès and Delfiner [1999].

The local trend $m_{n}^{(\mathrm{u})}(\mathbf{x})$ through the $P_{n}$ data points around $\mathbf{x}_{n}$ will be introduced in the Kriging equations, to ensure unbiasedness. The additional term is called the Lagrange term:

$$
m_{n}^{(\mathrm{u})}(\mathbf{x})=\sum_{k=0}^{2} u_{k, n}^{(\mathrm{u})} a_{k}(\mathbf{x}) .
$$

These Lagrange parameters $u_{k, n}^{(\mathrm{u})}$ also make the number of equations equal to the number of parameters, thereby enforcing a unique solution of the set of equations. (The values of the basis functions $a_{k}(\mathbf{x})$ at $\mathbf{x}_{p}$ are denoted $a_{k, p}$.) 
For $P_{n}$ data points and $K=3$ basis functions, this leads to the following set of $P_{n}+K$ equations at $\mathbf{x}_{n}$ :

$$
\begin{array}{rlrl}
\underline{c}_{p n}= & \sum_{q=1}^{P_{n}} w_{n q} \underline{c}_{p q}+\sum_{k=0}^{2} u_{k, n}^{(\mathrm{u})} a_{k, p}, & \forall p=1, \cdots, P_{n} ; \\
a_{k, n}=\sum_{p=1}^{P_{n}} w_{n p} a_{k, p}, & \forall k=0, \cdots, K-1 .
\end{array}
$$

The equation for $k=0$ implies that the Kriging weights add up to one, because $a_{0}=1$, independent of the positions $\mathbf{x}_{p}$. This does not exclude negative weights.

To describe equations (2.35) in vector notation, we introduce the $P_{n} \times P_{n}$ symmetrical covariance matrix $\underline{\mathbf{C}}$, containing the covariances $\underline{c}_{p q}$ for all $p$ and $q$, and variances $\underline{c}(0)$ on its main diagonal. To guarantee that this covariance matrix is positive definite, we cannot use zeros here for covariances between $\mathbf{x}_{p}$ and $\mathbf{x}_{q}$ at larger distances than $h^{(\mathrm{i})}$, but we use the values of the covariance function. Row $p$ and column $q$ of $\underline{\mathbf{C}}$ are denoted $\underline{\mathbf{c}}_{p}^{\mathrm{T}}$ and $\underline{\mathbf{c}}_{q}$. The $P_{n} \times 1$ vector $\mathbf{w}_{n}$ stores weights $\underline{w}_{p n}$. Also, we use the notation $\mathbf{a}_{p}$ and $\mathbf{u}_{n}^{(\mathrm{u})}$ for the $K \times 1$ vectors of basis function coefficients and Lagrange parameters at node $\mathbf{x}_{n}$, and $\mathbf{a}_{k}$ for the $P_{n} \times 1$ vector of coefficients of the basis function $k$ at positions $\mathbf{x}_{p}$. In vector notation, equation (2.35) becomes:

$$
\begin{array}{rlrl}
\underline{c}_{p n}= & {\left[\begin{array}{ll}
\underline{\mathbf{c}}_{p}^{\mathrm{T}} & \mathbf{a}_{p}^{\mathrm{T}}
\end{array}\right]\left[\begin{array}{c}
\mathbf{w}_{n} \\
\mathbf{u}_{n}^{(\mathrm{u})}
\end{array}\right],} & \forall p=1, \cdots, P_{n} ; \\
a_{k, n}=\mathbf{a}_{k}^{\mathrm{T}} \mathbf{w}_{n}, & \forall k=0, \cdots, K-1 .
\end{array}
$$

Let $P_{n} \times K$ matrix $\mathbf{A}$ contain the vectors $\mathbf{a}_{k}^{\mathrm{T}}$ in its rows and $\mathbf{a}_{p}$ in its columns, and let the $K \times K$ matrix $\mathbf{O}$ contain only zeros. The $P_{n} \times 1$ covariance vector $\underline{\mathbf{c}}_{n}$ stores the covariances $\underline{c}_{p n}$. We reformulate the linear system of equations $(2.36)$ as

$$
\left[\begin{array}{c}
\underline{\mathbf{c}}_{n} \\
\mathbf{a}_{n}
\end{array}\right]=\left[\begin{array}{cc}
\underline{\mathbf{C}} & \mathbf{A} \\
\mathbf{A}^{T} & \mathbf{O}
\end{array}\right]\left[\begin{array}{c}
\mathbf{w}_{n} \\
\mathbf{u}_{n}^{(\mathrm{u})}
\end{array}\right] .
$$

We use a positive definite covariance function for $\underline{\mathbf{C}}$, see Subsection 2.3.1, which guarantees that this submatrix is positive definite. However, there is no guarantee that the total matrix in equation (2.37) is positive definite. In the exceptional case that it is not, Chilès and Delfiner [1999] propose a solution based on two subsystems of positive definite matrices, which will not be discussed here. If it is positive definite, the Kriging weights and Lagrange parameters follow from

$$
\left[\begin{array}{l}
\underline{\mathbf{w}}_{n} \\
\underline{\mathbf{u}}_{n}^{(\mathrm{u})}
\end{array}\right]=\left[\begin{array}{cc}
\underline{\mathbf{C}} & \mathbf{A} \\
\mathbf{A}^{\mathrm{T}} & \mathbf{O}
\end{array}\right]^{-1}\left[\begin{array}{c}
\underline{\mathbf{c}}_{n} \\
\mathbf{a}_{n}
\end{array}\right] .
$$

These weights are used in equation (2.32) to calculate depth values. Their uncertainties are given in the next section. 


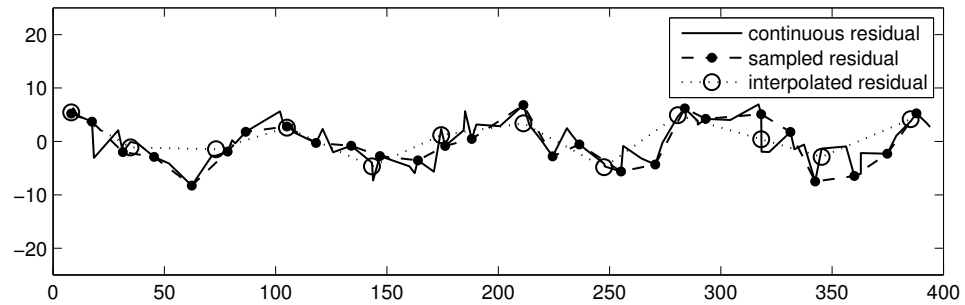

Figure 2.17: Sketch of the residual $r^{(\mathrm{hmv})}(\mathbf{x})$ (solid line), its discrete realization $\underline{r}^{(\mathrm{hmrv})}\left[\mathbf{x}_{p}\right]$ (black dots and dashed line), and its interpolated realization $\underline{r}^{(\mathrm{n})}\left[\mathbf{x}_{n}\right]$ (white circles and dotted line).

\subsubsection{Kriging uncertainty}

\section{The Kriging error}

The residuals $r^{(\mathrm{m})}(\mathbf{x})$ are not known at any position, however their approximated values $\underline{r}^{(\mathrm{hmrv})}$ are, at the positions $\mathbf{x}_{p}$. Interpolation regards these values $\underline{r}^{(\mathrm{hmrv})}\left[\mathbf{x}_{p}\right]$ as samples from the surface $\underline{r}^{(\mathrm{hmrv})}(\mathbf{x})$, as in figure 2.8. Thereby, it introduces a new error, the Kriging error $e^{(\mathrm{k})}$, at the interpolation position $\mathbf{x}_{n}$, see Figure 2.17.

The resulting value at the grid node is $\underline{r}^{(\mathrm{n})}$ :

$$
\underline{r}^{(\mathrm{n})}\left[\mathbf{x}_{n}\right]=\underline{r}^{(\mathrm{hmrv})}\left[\mathbf{x}_{n}\right]-\underline{e}^{(\mathrm{k})} .
$$

The sign of $\underline{e}^{(\mathrm{k})}$ is chosen negatively, because interpolation is a smoothing process, thereby reducing the size of the residual $\underline{r}^{\text {(hmrv) }}$. This means for instance that there will exist higher peaks and lower troughs than predicted by an interpolation procedure.

The interpolation error $e^{(\mathrm{k})}$ will be quantified by the Kriging variance $\underline{c}^{(\mathrm{k})}(0)$, to be derived below. The variances and covariances of the measurements at $\mathbf{x}_{p}$ propagate into the variance and covariances of the grid nodes at $\mathbf{x}_{n}$. We call this the propagated nodal covariance function $\underline{c}^{(\mathrm{n})}(\mathbf{h})$. Both contribute to the total covariance function at the nodes, which is still the stationary $\underline{c}^{(\mathrm{hmrv})}(\mathbf{h})$ :

$$
\underline{c}^{(\mathrm{hmrv})}(\mathbf{h})=\underline{c}^{(\mathrm{n})}(\mathbf{h})+\underline{c}^{(\mathrm{k})}(\mathbf{h}) .
$$

This situation is sketched in Figure 2.18. Note that the decreasing magnitude of the residuals of an interpolation process is visible here as a reduction of the variance $\sigma^{(\mathrm{hmv}) 2}$.

\section{Kriging onto a grid}

The calculation of a grid of $N$ interpolated depth values will not only enable the calculation of their propagated variances, see equation (2.32), but also their 


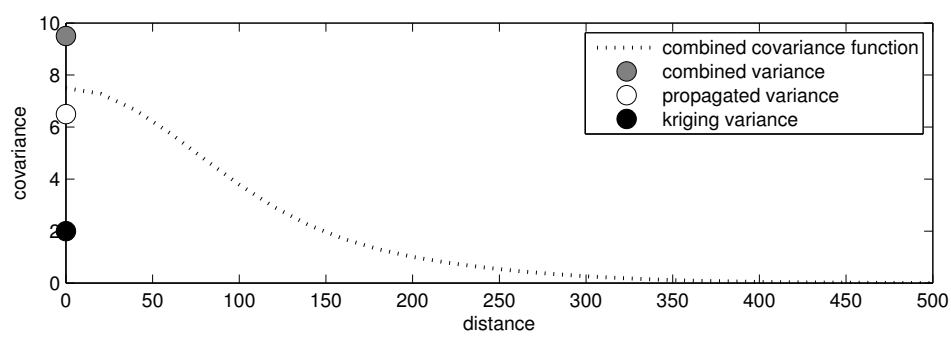

Figure 2.18: Covariance function for the combined residuals (dotted line), which equals the bottom graph in Figure 2.15. As this function is stationary, it is also valid for the propagated covariance between two nodes at $\mathbf{x}_{m}$ and $\mathbf{x}_{n}$. The propagated variance, the Kriging variance, and their combined variance are also shown.

mutual covariances. To calculate these propagated covariances of the grid, we first need to formulate how the depth values at the data points propagate into the depth values at the grid nodes, via the interpolation procedure. The values $\underline{r}^{\text {(hmrv) }}\left[\mathbf{x}_{p}\right]$ of all $P$ data points are collected into a $P \times 1$ vector $\underline{\mathbf{r}}_{p}$. The weight vectors $\underline{\mathbf{w}}_{n}^{\mathrm{T}}$ are the rows of $N \times P$ weight matrix $\underline{\mathbf{W}}$, after the insertion of zero weights for the data points further away than $h^{(\mathrm{i})}$. The $N \times K$ Lagrange matrix $\underline{\mathbf{U}}^{(\mathrm{u})}$ contains the Lagrange vectors $\underline{\mathbf{u}}_{n}^{(\mathrm{u}) \mathrm{T}}$. The $K \times 1$ vector o contains zeros only. The matrix $\underline{C}$ now has a size $P \times P$. The $N \times 1$ vector $\underline{\mathbf{r}}_{n}^{(\mathrm{n})}$ of interpolated residuals $\underline{r}^{(\mathrm{n})}\left[\mathbf{x}_{n}\right]$ and their matrix $\underline{\mathbf{C}}^{(\mathrm{n})}$ of propagated covariances are:

$$
\begin{aligned}
\underline{\mathbf{r}}_{n}^{(\mathrm{n})} & =\left[\begin{array}{ll}
\underline{\mathbf{W}} & \underline{\mathbf{U}}^{(\mathrm{u})}
\end{array}\right]\left[\begin{array}{c}
\underline{\mathbf{r}}_{p} \\
\mathbf{o}
\end{array}\right], \\
\underline{\mathbf{C}}^{(\mathrm{n})} & =\left[\begin{array}{ll}
\underline{\mathbf{W}} & \underline{\mathbf{U}}^{(\mathrm{u})}
\end{array}\right]\left[\begin{array}{cc}
\frac{\mathbf{C}}{\mathbf{A}} \\
\mathbf{A}^{\mathrm{T}} & \mathbf{O}
\end{array}\right]\left[\begin{array}{c}
\underline{\mathbf{W}}^{\mathrm{T}} \\
\underline{\mathbf{U}}^{(\mathrm{u}) \mathrm{T}}
\end{array}\right] .
\end{aligned}
$$

The interpolated residuals $\underline{r}^{(\mathrm{n})}\left[\mathbf{x}_{n}\right]$ and their variances $\sigma_{n}^{(\mathrm{n})}$, on the main diagonal of $\underline{\mathbf{C}}^{(\mathrm{n})}$, were originally introduced in equation (2.32).

We use $\underline{\mathbf{C}}^{(\mathrm{n})}$ to calculate the Kriging variances. This approach is also taken by Davis [2002]. The Kriging variance also follows from the minimum variance property, that is used by Chilès and Delfiner [1999] to derive the universal Kriging equations.

The matrix of covariances and base functions in equation (2.41) has already been used for the calculation of the weights, in equation (2.38). The covariance between nodes $\mathbf{x}_{m}$ and $\mathbf{x}_{n}$ is an element of covariance matrix $\underline{\mathbf{C}}^{(\mathrm{n})}$. Such an element is calculated as:

$$
\underline{c}_{m n}^{(\mathrm{n})}=\left[\begin{array}{ll}
\underline{\mathbf{w}}_{m}^{\mathrm{T}} & \underline{\mathbf{u}}_{m}^{(\mathrm{u})}
\end{array}\right]\left[\begin{array}{cc}
\underline{\mathbf{C}} & \mathbf{A} \\
\mathbf{A}^{\mathrm{T}} & \mathbf{O}
\end{array}\right]\left[\begin{array}{c}
\underline{\mathbf{w}}_{n} \\
\underline{\mathbf{u}}_{n}^{(\mathrm{u})}
\end{array}\right] .
$$


Using equation (2.38), this equation becomes

$$
\underline{\mathbf{c}}_{m n}^{(\mathrm{n})}=\left[\begin{array}{ll}
\underline{\mathbf{w}}_{m}^{\mathrm{T}} & \underline{\mathbf{u}}_{m}^{(\mathrm{u}) \mathrm{T}}
\end{array}\right]\left[\begin{array}{l}
\underline{\mathbf{c}}_{n} \\
\mathbf{a}_{n}
\end{array}\right] .
$$

A propagated variance element $\underline{\underline{n}}_{n}^{(\mathrm{n}) 2}$ is calculated correspondingly.

Using equation (2.40), the covariance function for the Kriging uncertainties is:

$$
\underline{c}^{(\mathrm{k})}(\mathbf{h})=\underline{c}^{(\mathrm{hmrv})}(\mathbf{h})-\underline{c}^{(\mathrm{n})}(\mathbf{h}) .
$$

Covariances $\underline{c}^{(\mathrm{n})}(\mathbf{h})$ are calculated from equation (2.43). Because all covariances for $h>0$ are defined stationary, as assumed in equation (2.33), the outcome of equation $(2.43)$ equals $\underline{c}^{(\mathrm{hmrv})}(\mathbf{h})$, and therefore all Kriging covariances are zero, for $m \neq n$ :

$$
\underline{c}^{(\mathrm{k})}(\mathbf{h})=0, h>0 .
$$

However, if $m=n$, we do not assume the propagated variance of a grid node $\underline{c}^{(\mathrm{n})}(0)$ to equal the variance of the residuals $\underline{\sigma}^{(\mathrm{hmrv}) 2}$. The differences are the Kriging variances, which are, using equation $(2.43)$ :

$$
\underline{\sigma}_{n}^{(\mathrm{k}) 2}=\underline{\sigma}^{(\mathrm{hmrv}) 2}-\mathbf{w}_{n}^{\mathrm{T}} \mathbf{c}_{n}-\mathbf{u}_{n}^{(\mathrm{u}) \mathrm{T}} \mathbf{a}_{n} .
$$

Clearly, the Kriging variance has to be interpreted as a nugget function:

$$
\underline{c}^{(\mathrm{k})}(\mathbf{h})>0, h=0 .
$$

If the interpolation is accurate $\left(\underline{\sigma}^{(\mathrm{k}) 2}\right.$ is small), a large part of the stationary variance $\underline{\sigma}^{(\mathrm{hmrv}) 2}$ at $\mathbf{x}_{n}$ is due to the propagated variance $\underline{\sigma}^{(\mathrm{n}) 2}$. It is caused by the morphological deviations from the trend, as well as the horizontal and vertical errors of the surrounding data points that propagate into the variance at $\mathbf{x}_{n}$ due to their correlations. However, if the interpolation is inaccurate, there is not much correlation with the data points, which means that the propagated influence of the morphology and the errors cannot be large. Instead, the Kriging error is large, to account for the stationary variance $\underline{\sigma}^{(\mathrm{hmrv}) 2}$ at $\mathbf{x}_{n}$. The situation is visualized in Figure 2.18. See Section 2.4.4 for an example of the propagated and the Kriging variances.

\section{The minimum and maximum Kriging variance}

To get insight in the values that we can expect for the Kriging variance, we discuss the two extreme situations of full covariance and no covariance between points. We denote the Kriging variances of the two cases as $\underline{\sigma}_{\min }^{(\mathrm{k}) 2}$ and $\underline{\sigma}_{\max }^{(\mathrm{k}) 2}$ respectively. Thereby, we use the equations that we just derived here above.

In case all covariances approximate their maximum $\underline{c}_{0}^{(\mathrm{hmrv})}$, the vector product $\underline{\mathbf{w}}_{n}^{\mathrm{T}} \underline{\mathbf{c}}_{n}$ also approximates $\underline{c}_{0}^{(\mathrm{hmrv})}$. Therefore, equation (2.46) gives:

$$
\begin{aligned}
\underline{\sigma}_{\min }^{(\mathrm{k}) 2} & =\underline{\sigma}^{(\mathrm{hmrv}) 2}-\underline{c}_{0}^{(\mathrm{hmrv})}-\underline{\mathbf{u}}_{n}^{(\mathrm{u}) \mathrm{T}} \mathbf{a}_{n} \\
& =\underline{\gamma}^{(\mathrm{hmrv})}(0)-\underline{\mathbf{u}}_{n}^{(\mathrm{u}) \mathrm{T}} \mathbf{a}_{n} .
\end{aligned}
$$


Provided that the Lagrange term is negligible, we conclude that the minimum Kriging variance equals the nugget of the covariance function: $\underline{\sigma}^{(\mathrm{k}) 2} \approx$ $\underline{\gamma}^{(\mathrm{hmrv})}(0)$.

In case all covariances approximate zero, the vector product $\underline{\mathbf{w}}_{n}^{\mathrm{T}} \underline{\mathbf{c}}_{n}$ also approximates zero. It follows from equation (2.46) that

$$
\underline{\sigma}_{\max }^{(\mathrm{k}) 2}=\underline{\sigma}^{(\mathrm{hmrv}) 2}-\underline{\mathbf{u}}_{n}^{(\mathrm{u}) \mathrm{T}} \mathbf{a}_{n} .
$$

Provided that the Lagrange term is negligible, we conclude that the maximum Kriging variance equals the variance: $\underline{\sigma}^{(\mathrm{k}) 2} \approx \underline{\sigma}^{(\mathrm{hmrv}) 2}$.

The above leaves the question if the Lagrange term $m_{n}^{(\mathrm{u})}\left(\mathbf{x}_{n}\right)=\underline{\mathbf{u}}_{n}^{(\mathrm{u}) \mathrm{T}} \mathbf{a}_{n}$ really is negligible. The three Lagrange parameters $\underline{\mathbf{u}}_{n}^{(\mathrm{u})}$, calculated via equation (2.38), represent a mean residual, and the two slopes of the tangential plane. The closer the residuals around $\mathbf{x}_{n}$ are to zero, and the less they change, the smaller the Lagrange term is. In practice, the Lagrange parameters are so small that the product $\underline{\mathbf{u}}_{n}^{(\mathrm{u}) \mathrm{T}} \mathbf{a}_{n}$ does not play a significant role. See Section 2.4.4 for an example of the Lagrange terms.

\subsubsection{Kriging of a bathymetric survey}

As an example, Figures 2.19 to 2.23 show the results of the geostatistical interpolation of a critical area of the Selected Track. From these figures, it becomes clear that the biggest source of interpolation uncertainty is an uneven distribution of data points around a node, as happens at the boundary of the area. The Kriging variances all exceed the nugget effect $\underline{\gamma}(0)$ of the covariance function, and they are all smaller than the total variance $\underline{c}(0)$. Both are visible in Figure 2.14. A comparison of Figure 2.14 with Figure 2.20 shows that all nodes have been interpolated relatively accurately.

The application of geostatistics to hydrography is common. Most usage assumes the sea floor does not have a trend, and therefore apply ordinary Kriging. Herzfeld [1989] applies universal Kriging, because she cannot make this assumption. Some apply geostatistics to formulate how accurately the sea floor is known between the sounding lines of single-beam surveys [Calder, 2006; Kielland and Tubman, 1994; Velberg, 1993; Kielland et al., 1992]. Subsequently, it is possible to formulate recommendations on line spacing [Bouwmeester and Heemink, 1993; Kielland and Dagbert, 1992]. Others use geostatistics to calculate a continuous model of the sea floor from point data for mapping [Chilès, 1976; Chilès and Chauvet, 1975], even in case of strong anisotropies [Te Stroet and Snepvangers, 2005]. Such a continuous model could also be used for volume calculations [Slobbe, 2005]. Bottelier et al. [2005] use Kriging for outlier detection in multi-beam data sets, and Van Dijk et al. [2008], Lindenbergh et al. [2006] and Laban [2005] apply it in a sand wave area, to filter rhythmic features at certain scales from multi-beam data. The use of geostatistics in hydrography is approved by the IHO, as a bathymetric model [International Hydrographic Organization, 2008a].

Using geostatistics, we could calculate the nodes as densely as we like. A higher density of known points means a better knowledge of the discrete func- 


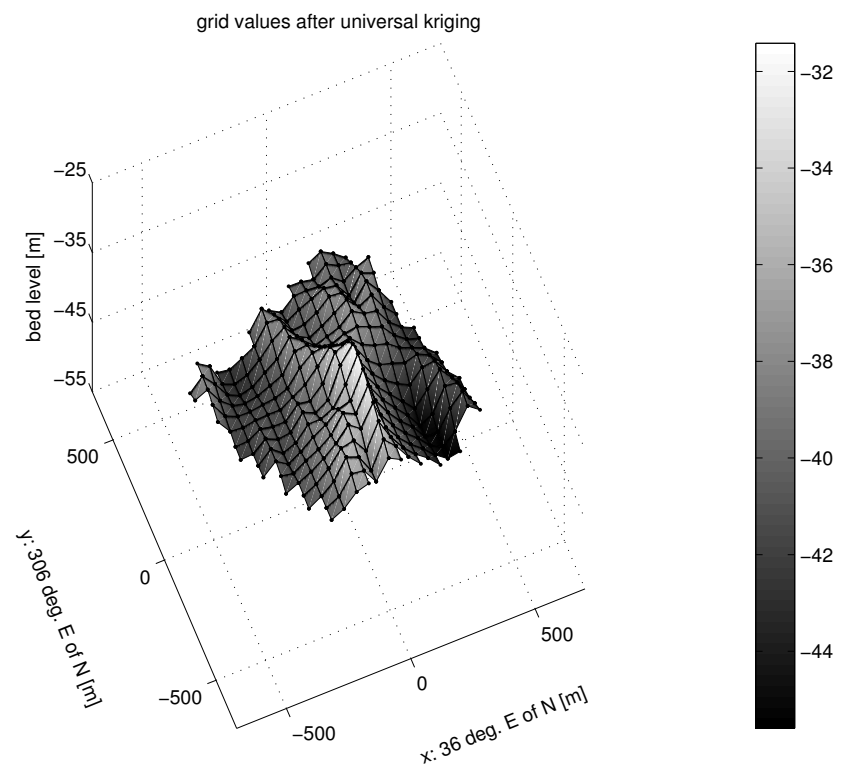

Figure 2.19: Results of the Kriging process for the May 2000 survey of critical area G: kriged depth values $\underline{d}_{n}$. The $x$-axis coincides with the direction of highest variability $\underline{\theta}^{(\mathrm{x})}$, which equals the direction of the sand wave pattern, and the $y$-axis with the direction of lowest variability $\underline{\theta}^{(\mathrm{y})}$, which equals the direction of the sand wave crests. These directions are the directions determined in Figure 2.13.

tion. To prevent the incorrect conclusion of very good knowledge of this function, based on a few data points, it is not advisable to sample at a higher density than the original data. This problem is discussed for hydrography by Slobbe [2005].

As discussed in Section 2.3.1, the maximum relevant distance of a data point is called the range. By setting the range equal to the inflection point of the Gaussian covariance function, we make a rather rigorous choice. This reduces the number of data points, while still a good nodal accuracy is obtained. Limiting the Kriging range has two advantages. The first advantage is that it speeds up the interpolation process. The second advantage is that isotropy can be assumed on small distances. However, for a good fit of $c(h)$, it is necessary to calculate $c[h]$ up to a large distance. A large maximum point distance means smooth interpolated values, so the preservation of details might be another reason to limit the Kriging distance.

In hydrography, the sea floor is often represented using $5 \times 5$ meter bins. The sea floor is assumed horizontal within such a bin. This assumption allows us not to interpolate, if a data point is available at a shorter distance than $5 \mathrm{~m}$ from the node. Instead, we assign the value of the closest data point and its measurement uncertainty. We only take this approach if a value is available for 


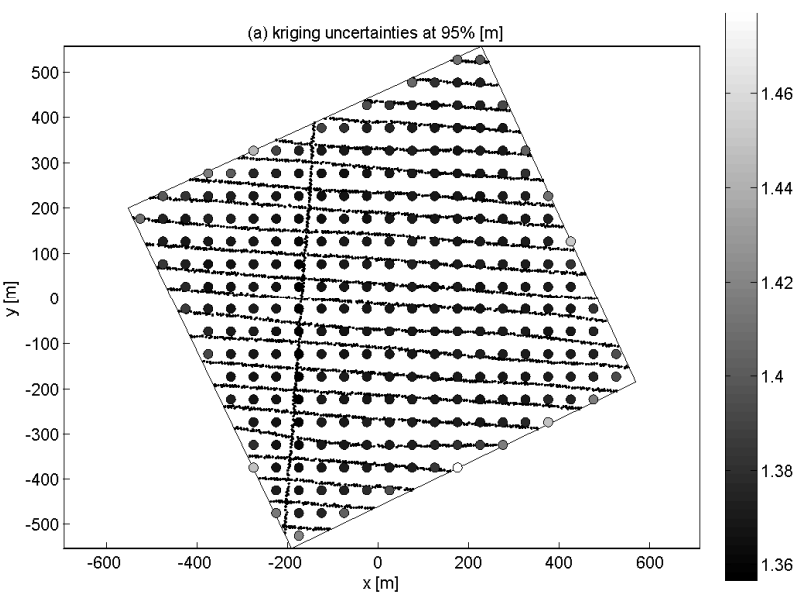

Figure 2.20: Results of the Kriging process for the May 2000 survey of critical area G: Kriging uncertainties for the $95 \%$ confidence limit $1.96 \underline{\sigma}_{n}^{(\mathrm{k})}$. Also, the original data points and the boundaries of the area are shown. The Kriging variances are all larger than the nugget effect $\gamma(0)$ of the covariance function, and all smaller than the total variance $\underline{c}(0)$. These values are given in Figure 2.14. The sum of the two uncertainties $\underline{\sigma}_{n}^{(\mathrm{k}) 2}$ and $\underline{\sigma}_{n}^{(\mathrm{n}) 2}$ is $\underline{c}(0)$.

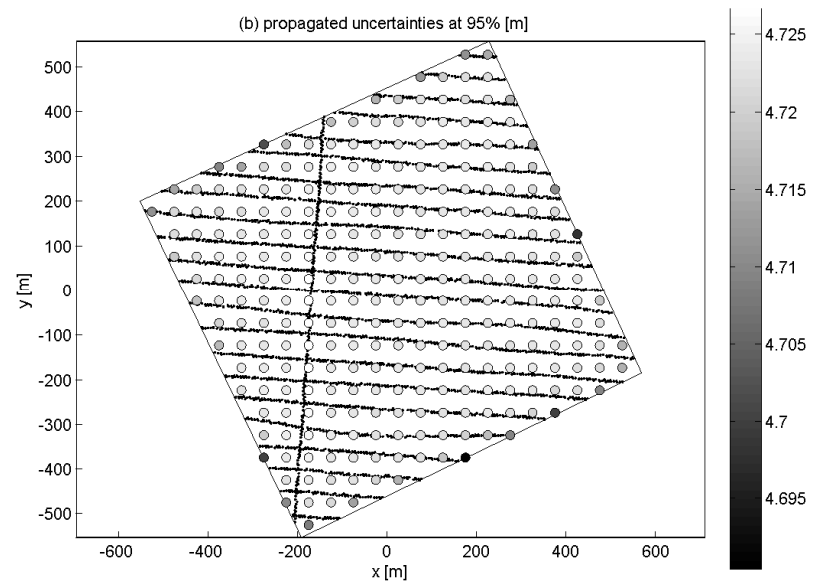

Figure 2.21: Results of the Kriging process for the May 2000 survey of critical area G: the propagated uncertainties for the $95 \%$ confidence limit $1.96 \underline{\sigma}_{n}^{(\mathrm{n})}$. Also, the original data points and the boundaries of the area are shown. The sum of the two uncertainties $\underline{\sigma}_{n}^{(\mathrm{k}) 2}$ and $\underline{\sigma}_{n}^{(\mathrm{n}) 2}$ is $\underline{c}(0)$. 


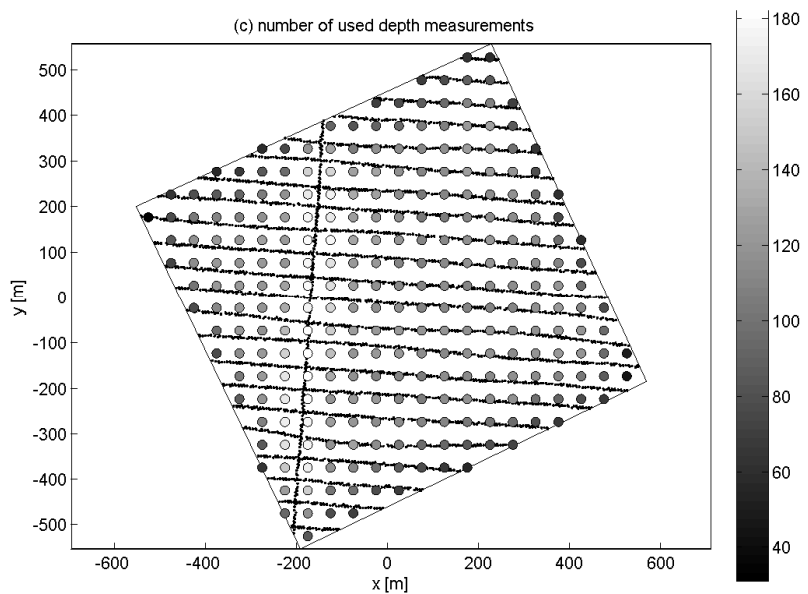

Figure 2.22: Results of the Kriging process for the May 2000 survey of critical area G: the number of used depth measurements $P_{n}$. Also, the original data points and the boundaries of the area are shown.

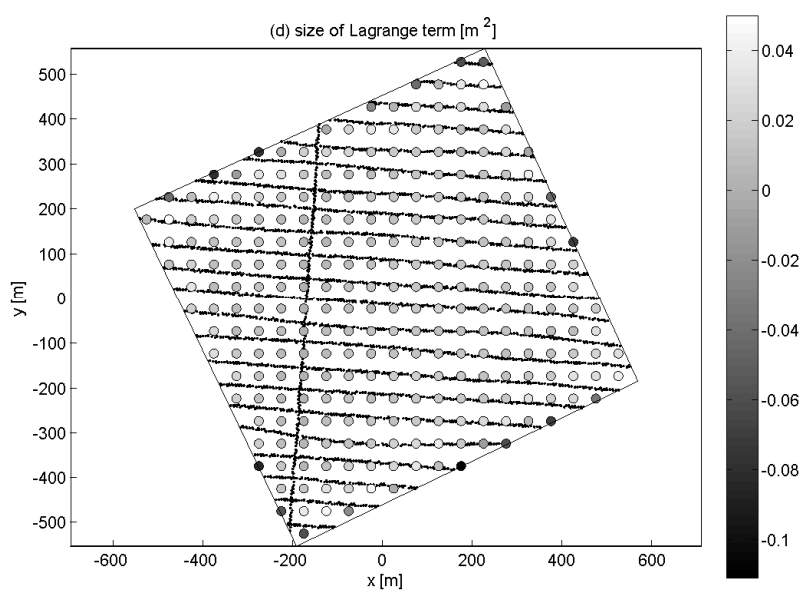

Figure 2.23: Results of the Kriging process for the May 2000 survey of critical area G: the Lagrange terms $m_{n}^{(\mathrm{u})}\left(\mathbf{x}_{n}\right)$. Also, the original data points and the boundaries of the area are shown. 
each bin. In case of such a survey with full coverage of the sea floor, Kriging is not necessary at all.

\subsection{Conclusion}

If bathymetry is represented by a continuous surface, interpolation of the depth measurements is necessary to calculate depth values anywhere on this surface, like a regular grid. However, interpolation of bathymetric data, already affected by horizontal and vertical measurement errors, results in an additional interpolation error. The application of Universal Kriging calculates a variance for this interpolation error, and is suitable for areas with a slope and a sand wave pattern. Universal Kriging does not introduce additional covariances between the depth values at the interpolated positions. The assumption of second order stationarity plays a central role, limiting the size of the area to be interpolated. 


\title{
Chapter 3
}

\section{The estimation of sea floor dynamics from bathymetric surveys of a sand wave $\operatorname{area}^{\star}$}

\begin{abstract}
The analysis of series of offshore bathymetric surveys provides insight into the morphodynamics of the sea floor. This knowledge helps to improve resurvey policies for the maintenance of port approaches and nautical charting, and to validate morphodynamic models. We propose a method for such an analysis that is based on statistical testing theory, particularly aimed at the detection of dynamics in areas with tidal sand waves. The method explicitly considers the uncertainty of every measured depth value, resulting in parameter estimates for the sea floor dynamics, and their uncertainty. We apply the method to sea floor representations both with and without a sand wave pattern. A test scenario is set up, consisting of a survey of an existing area in the Southern North Sea, for which dynamics are simulated. The results show that the proposed method detects different types of sea floor dynamics well, leading to satisfactory estimates of the corresponding parameters.
\end{abstract}

\subsection{Introduction}

The sea floor of shallow seas is dynamic. For example, rhythmic morphological patterns on sandy beds can change in height and migrate [e.g. Wright, 1992; Terwindt, 1971], thereby possibly endangering navigation through busy shipping lanes. Such patterns exist on many scales [Knaapen et al., 2001]. In this paper we consider tidal sand waves which are characterized by a wavelength of hundreds of meters, a height of several meters, and a migration rate of up to twenty meters per year [Van Dijk et al., 2008; Van Dijk and Kleinhans, 2005; Knaapen et al., 2001].

The evolution of the sea floor can be interpreted from a series of echo sounder surveys, as has been attempted for the Netherlands Continental Shelf by many authors [recently e.g. Van Dijk et al., 2008; Buijsman, 2007; Knaapen, 2005]. The stochastic character of surveyed depth values of the sea floor complicates

^ This chapter was published as L. L. Dorst, P. C. Roos, S. J. M. H. Hulscher, and R. C. Lindenbergh [2009]. The Estimation of Sea Floor Dynamics from Bathymetric Surveys of a Sand Wave Area, J. of Applied Geodesy, 3(3), 97-120. 
the analysis of its evolution: even modern survey techniques reveal depth values with an uncertainty of a few decimeters at best [Wells and Monahan, 2002], due to random and systematic errors in the measurement processes.

Frequent bathymetric surveys of shallow seas are necessary to support the maintenance of port approaches, to produce reliable nautical charts, and to support coastal management decisions. The analysis of past surveys aids the planning of resurvey frequencies. Examples of survey plans are available on the internet [NOAA Office of Coast Survey, 2008] and in the literature [De Oliveira et al., 2007; Dehling, 2006; Whatrup et al., 2005]. In our opinion, resurvey planning is best served by applying a method that can distinguish between different types of dynamics in an area. One may e.g. wish to differentiate between trends in large-scale sea floor depth and trends in crest height of a sand wave pattern. It is thereby necessary to separate true sea floor deformation from measurement inaccuracies, or to establish whether a new survey confirms previous surveys.

The quantification of the uncertainty of surveyed depth values is a subject of growing interest in the hydrographic community [e.g. Calder, 2006; Dorst, 2004; Hare, 1995], since dealing with uncertainties is crucial to control the quality of the survey during the process and to interpret bathymetric data. Therefore, the method should recognize the stochastic character of surveys. Other requirements are that such a method detects the type of dynamics in an area, estimates the sizes of the detected dynamics well, and provides the uncertainty associated with those estimates.

There are a few authors that have taken measurement uncertainty into account. Wüst [2004] presents a method that is able to estimate local linear trends in depth, using uncertainties. Knaapen et al. [2005] and Lindenbergh et al. [2007] developed his method further. The genetic method of Knaapen and Hulscher [2002] is capable of estimating trends in amplitude. Several authors have attempted to remove horizontal inaccuracies in sand wave migration studies, using markers [recently e.g. Knaapen, 2005].

In this paper, we propose deformation analysis as a method to analyze a series of surveys of the sea floor, particularly in the presence of a sand wave. e.g. Lindenbergh et al. [2007] and Dorst [2004] described this application before in less detail. The application of this method at the Netherlands Hydrographic Service was discussed before at a conceptual level [Dorst, 2005]. The novelties presented here are that this application of deformation analysis is described in combination with variance component estimation, and in combination with the estimation of wavelength. A third novelty is that the specific application to one-dimensional cross-sections of an area is studied.

An overview of literature on deformation analysis is given by Chrzanowski [2006]. This type of analysis applies statistical adjustment theory and testing theory to a series of surveys of deformable objects. Adjustment theory involves parameter estimation, and testing theory involves hypothesis testing. These statistical concepts are applied to the processing of surveys by e.g. Teunissen [2000, 2001] or Koch [1999].

The proposed method is described in Section 3.2. A test scenario that is described in Section 3.3 illustrates the validity of this approach. The results of 


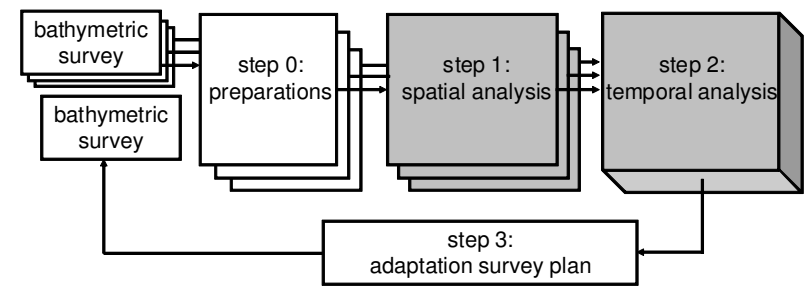

Figure 3.1: Overview of the procedure to analyze a series of surveys. This paper focuses on the shaded steps.

the analysis of this scenario are discussed in Section 3.4. Sections 3.5 and 3.6 contain the discussion and conclusions, respectively.

\subsection{The method for the estimation of sea floor dynamics}

\subsubsection{Introduction}

The method consists of several steps (Figure 3.1): the preparation of a series of bathymetric surveys (step 0), see Section 3.2.2; the actual analysis (steps 1 and 2); and the adaptation of the survey policy (step 3, beyond the scope of this article). This adaptation influences the availability of future surveys, and thereby the moment a new analysis can be done.

The analysis results in a separation of the morphological characterization from the residual variation, the concept of which is given in Section 3.2.3. Alternative characterizations are specified in Section 3.2.4, and selected in Section 3.2.5. The residuals are described in Section 3.2.6.

\subsubsection{Input}

\section{Overview of the input}

Our analysis method requires an input consisting of the following five quantities:

1. fixed positions $\mathbf{x}_{p}=\left(x_{p}, y_{p}\right)$ at points $p=1,2, \cdots, P$;

2. times $t_{s}$ for the surveys $s=1,2, \cdots, S$;

3. depth values $\underline{d}_{p, s}$ for each point $p$ and each survey $s$;

4. error variances $\sigma_{p, s}^{(\mathrm{e}) 2}$ for each point $p$ and each survey $s$;

5. covariances $c_{p, q, s}^{(\mathrm{e})}$ between the errors at $\mathbf{x}_{p}$ and $\mathbf{x}_{q}$ during survey $s$. 
For the properties of the variance and covariances of error $\underline{e}_{p, s}$, see Appendix 3.A.

A logical choice for the set of points is a rectangular grid with axes in the $(x, y)$-directions and a sufficiently high resolution to prevent aliasing effects of possible sand wave patterns. Due to computational constraints, the grid can in practice not be very dense, though. The grid axis $x$ coincides with the direction of the highest variability, which is determined using the DIGIPOL method [Van Munster et al., 1995; RIKZ, 1997; De Koning, 2007]. This is done to ensure that the direction of a sand wave pattern, if present, coincides with the $x$ direction, and the grid axis $y$ is thus parallel to the direction of the crests. The spatial extent of the area is restricted by the assumption that both the spatial covariance relations and the morphodynamics are uniform within the area. The spatial covariances are discussed in Appendix 3.A.

Each node must be assigned a depth value and a variance for each survey. Such an assignment procedure is evident for surveys that fully cover the sea floor, but requires interpolation in other cases. Kriging is a commonly accepted method for bathymetric interpolation (Chapter 2). A recently proposed alternative is the use of Combined Uncertainty and Bathymetry Estimation (CUBE) [Calder, 2003]. The interpolation procedure allows us to combine surveys of single-beam echo sounders and surveys of multi-beam echo sounders. The input requirements are further discussed in Section 3.5.1.

\section{Vectorization of the input}

We use a column vector $\underline{\mathbf{d}}$ for all depth values $\underline{d}_{p, s}$ at all $p$ and all $s$, in which the order is not relevant. (Vectors are printed in bold lower case letters.) We do the same for the error $\underline{e}_{p, s}$ in $\underline{\mathbf{e}}$ and the residual $\underline{r}_{p, s}^{(\mathrm{em})}$ in $\underline{\mathbf{r}}$. All these vectors have size $M \times 1$, where $M=P \bar{S}$ is the total number of depth measurements. We also introduce the $S \times 1$ column vector $\mathbf{t}$ of survey times relative to reference time $t_{\text {ref }}: \mathbf{t}=\left[t_{1}-t_{\text {ref }}, t_{2}-t_{\text {ref }}, \cdots, t_{S}-t_{\text {ref }}\right]^{\mathrm{T}}$. (A transpose is denoted by a superscripted T.)

Finally, we define the covariance matrix of the measured depth values as $\mathbf{C}_{d}$, with the variances on its main diagonal, and covariances on its symmetric off-diagonal elements. (Matrices are printed in bold upper case letters.) In case of variances and covariances for the measurement errors $\underline{e}_{p, s}$, matrix $\mathbf{C}_{d}^{(\mathrm{e})}$ contains the variances $\sigma_{p, s}^{(\mathrm{e}) 2}$ and the auto-covariances $c_{p, s_{1}, q, s_{2}}^{(\mathrm{e})}$ between the depth values at position $\left(x_{p}, y_{p}\right)$ during the survey at $t_{s_{1}}$, and position $\left(x_{q}, y_{q}\right)$ during the survey at $t_{s_{2}}$. The measurement processes cause no correlation between surveys, so $c_{p, s_{1}, q, s_{2}}=0$ if $s_{1} \neq s_{2}$. If $s_{1}=s_{2}=s, c_{p, q, s}$ is calculated from function $c_{s}(\mathbf{h})$, where covariance $c_{s}$ depends on position difference $\mathbf{h}$ between $\mathbf{x}_{p}$ and $\mathbf{x}_{q}$.

\subsubsection{Sea floor characterization and its residuals}

Consider a three-dimensional Cartesian coordinate system with horizontal axes $x$ and $y$, and an upward $z$ axis. The sea floor at time $t$ is given by the surface $z=-d(x, y, t)$. The measurement and interpolation of the depth values $d(x, y, t)$ 
introduce an error $\underline{e}(x, y, t)$ :

$$
\underline{d}(x, y, t)=d(x, y, t)+\underline{e}(x, y, t) .
$$

(We underline all realizations of parameters influenced by stochastic measurement and interpolation inaccuracies.) The actual error $\underline{e}(x, y, t)$ associated with $\underline{d}(x, y, t)$ is unknown, but its variance $\sigma^{(\mathrm{e}) 2}(x, y, t)$ is often quantified using a statistical procedure, see e.g. Calder [2006]; Hare [1995] for measured depth values, and e.g. Calder [2006]; Velberg [1993] for interpolated depth values. Usually, it is assumed that the errors have a Gaussian distribution, with an expected value of zero, and a variance expressed by the $95 \%$ probability value $1.96 \sigma^{(\mathrm{e})}(x, y, t)$.

Each point on the measured depth surface $\underline{d}(x, y, t)$ can be considered a superposition of a morphological characterization $m(x, y, t)$ that we consider to be a deterministic trend function, and the remaining morphological residual $r^{(\mathrm{m})}(x, y, t)$ that we consider to be a random function. The total residual $\underline{r}^{(\mathrm{em})}(x, y, t)$ is the sum of the morphological residual and the error. This enables us to express equation (3.1) as:

$$
\underline{d}(x, y, t)=m(x, y, t)+\underline{r}^{(\mathrm{em})}(x, y, t) .
$$

Just like the depth function $\underline{d}(x, y, t)$ is only known through its realization $\underline{d}_{p, s}$, we will also work with discrete sets $m_{p, s}, \underline{r}_{p, s}^{(\mathrm{em})}$ and $\underline{e}_{p, s}$ for $m(x, y, t)$, $\underline{r}^{(\mathrm{em})}(x, y, t)$ and $\underline{e}(x, y, t)$ respectively.

The expected value of $\underline{r}^{(\mathrm{em})}(x, y, t)$ is zero, and a measure of its variability, or dispersion, is the variance $\sigma_{r}^{2}(x, y, t)$ :

$$
\mathrm{E}\left\{\underline{r}^{(\mathrm{em})}(x, y, t)\right\}=0 ; \mathrm{D}\left\{\underline{\underline{r}}^{(\mathrm{em})}(x, y, t)\right\}=\sigma_{r}^{2}(x, y, t) .
$$

The mathematical operator for the expected value is denoted by $\mathrm{E}\{\}$, and for dispersion by $\mathrm{D}\{\}$, defined as

$$
\left.\mathrm{D}\left\{\underline{r}^{(\mathrm{em})}(x, y, t)\right\}=\mathrm{E}\left\{\underline{r}^{(\mathrm{em})}(x, y, t)-\mathrm{E}\left\{\underline{r}^{(\mathrm{em})}(x, y, t)\right\}\right)^{2}\right\} .
$$

The characterization is assumed deterministic, which means that it has no dispersion, $\sigma_{m}^{2}=0$, and therefore the dispersion of depth equals the dispersion of the residuals, $\sigma_{d}^{2}=\sigma_{r}^{2}$. Depth is then represented using the trend function and the variance function of the residuals:

$$
\mathrm{E}\{\underline{d}(x, y, t)\}=m(x, y, t) ; \mathrm{D}\{\underline{d}(x, y, t)\}=\sigma_{r}^{2}(x, y, t) .
$$

We call this combination of a characterization and its dispersion a representation $R$.

\subsubsection{Morphological characterization}

\section{Characterization in space}

In this section, we characterize the sea floor by a one-dimensional approach in $x$ direction, i.e. considering grid lines of constant $y$. Therefore, depth is expressed as $d=d(x, t)$, with a positive sign in negative $z$-direction. 
We introduce several alternative representations $R$ to characterize the complexity of the morphology $m_{s}(x)$ of the sea floor during survey $s$. There are four levels of complexity for the representation $R: R^{(0)}, R^{(1)}, R^{(2)}$, and $R^{(1+2)}$, see Figure 3.2. We assume that all surveys have the same level of complexity, and that the wavelength in $R^{(2)}$ and $R^{(1+2)}$ is constant.

The simplest characterization of the sea floor is a horizontal surface, the zero-level representation $R^{(0)}: d_{s}^{(\mathrm{b})}$ is constant. The only parameter involved is the bed level $d^{(\mathrm{b})}$ :

$$
R^{(0)}: m_{s}(x)=d_{s}^{(\mathrm{b})} .
$$

The three alternatives $R^{(a)}$ assume that depth varies with position, with $a \in$ $\{1,2,1+2\}$. An addition to a characterization to create a more complex one is called an extension. In the representation $R^{(1)}$, we extend $R^{(0)}$ using the slope parameter $\psi=-\Delta z / \Delta x$ to model a linearly sloping characterization:

$$
R^{(1)}: m_{s}(x)=d_{s}^{(\mathrm{b})}+\psi_{s} x .
$$

We call both the representations of equations (3.6) and (3.7) planar, although in the absence of a second dimension $y$ they represent straight lines. This is done for similarity with the two-dimensional approach.

As an alternative for $R^{(1)}$, we represent a rhythmic pattern using a cosine function with parameters amplitude $A$ and crest position $\xi$. The pattern has a constant wavelength $L$, which is determined during the testing procedure:

$$
m_{s}(x)=d_{s}^{(\mathrm{b})}-A_{s} \cos \left(2 \pi\left(x-\xi_{s}\right) / L\right) .
$$

In this equation, the relation between the morphological characterization $m_{s}(x)$ and crest position $\xi_{s}$ is nonlinear. A minus sign is introduced because the crest is defined by the minimum depth. To obtain a linear expression, we replace the original sand wave parameters $A_{s}$ and $\xi_{s}$ by the linear provisional sand wave parameters $A_{s}^{(\mathrm{c})}$ and $A_{s}^{(\mathrm{s})}$. They are related as:

$$
A_{s}^{(\mathrm{c})}=A_{s} \cos \kappa \xi_{s}, A_{s}^{(\mathrm{s})}=A_{s} \sin \kappa \xi_{s}
$$

with wave number $\kappa=2 \pi / L$, see also Appendix 3.B. The second-level characterization of representation $R^{(2)}$ thus becomes:

$$
R^{(2)}: m_{s}(x)=d_{s}^{(\mathrm{b})}-A_{s}^{(\mathrm{c})} \cos \kappa x-A_{s}^{(\mathrm{s})} \sin \kappa x .
$$

Also, we allow a combination of $R^{(1)}$ and $R^{(2)}$, denoted $R^{(1+2)}$ :

$$
R^{(1+2)}: m_{s}(x)=d_{s}^{(\mathrm{b})}+\psi_{s} x-A_{s}^{(\mathrm{c})} \cos \kappa x-A_{s}^{(\mathrm{s})} \sin \kappa x .
$$

Because the pattern on the sea floor seldom takes the shape of a sine function, the amplitude $A$ and the crest position $\xi$ of $R^{(2)}$ are hard to interpret. Instead, we use the parameters amplitude factor $A^{\prime}=A / A_{\text {ref }}$ and migration $\xi^{\prime}=\xi-\xi_{\text {ref }}$ with respect to the estimated amplitude and crest position of a reference survey, e.g. for survey $s=1$, see also Appendix 3.B. We call those parameters the final 


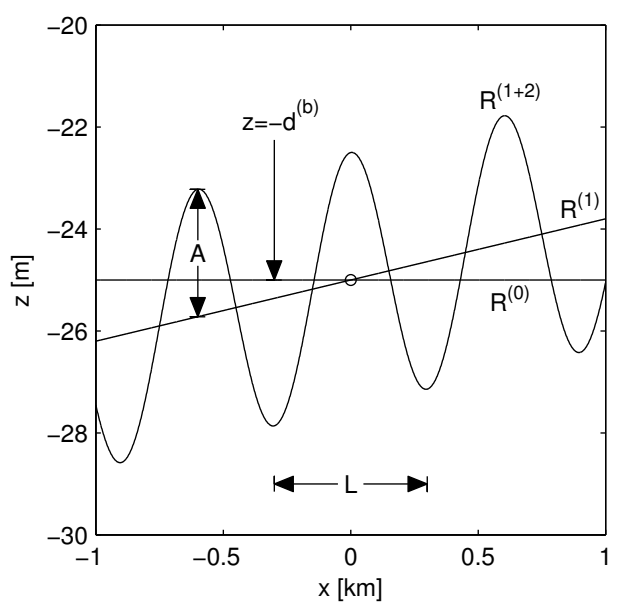

Figure 3.2: Definition sketch of the sea floor characterizations, showing some of the parameters that are used in the testing procedure.

sand wave parameters, they have a physical meaning for any pattern that is present in a series of surveys.

A general linear expression for the characterization $m_{s}(x)$ is by the inner product of a parameter vector $\mathbf{u}$ and a coefficient vector $\mathbf{a}$ :

$$
m_{s}(x)=\mathbf{a}^{\mathrm{T}} \mathbf{u}_{s} \text {. }
$$

A characterization of survey $s$ in step 1 is written as in equation (3.12), using $\mathbf{u}_{s}$ for a parameter $d^{(\mathrm{b})}, \psi, A^{(\mathrm{c})}$ or $A^{(\mathrm{s})}$, and using a for the coefficients. Each coefficient $a$ equals the value of a basis function $a(x)$ at $x: 1, x, \cos \kappa x$ or $\sin \kappa x$, respectively. Both $\mathbf{u}_{s}$ and $\mathbf{a}$ are $U \times 1$ column vectors, with $U$ the number of parameters.

Because all surveys use the same $U$ parameters, all vectors a are the same for every survey $s$. All parameter vectors $\mathbf{u}_{s}$ are different, as there are always some differences between the surveyed depth values, resulting in $U S$ parameters to describe the sea floor during $S$ surveys. In step 1 , it is not yet clear if parameter $u$ is static or dynamic. In step 2, we will detect if dynamics are present for each parameter $u$, and if so, what the structure of the dynamics is.

\section{Characterization in time}

The spatial representation introduced above describes the sea floor during a single survey $s$. To find significant dynamics, we formulate a static representation as $R_{0}: \mathrm{E}\{d\}=m(x)$ (Figure 3.3a). Also, we formulate alternative representations as $R_{a}: \mathrm{E}\{d\}=m(x, t)$, which extend the static characterization to allow for specific changes of a sea floor parameters $u$ in time. All characterizations remain linear, as in equation (3.12). 


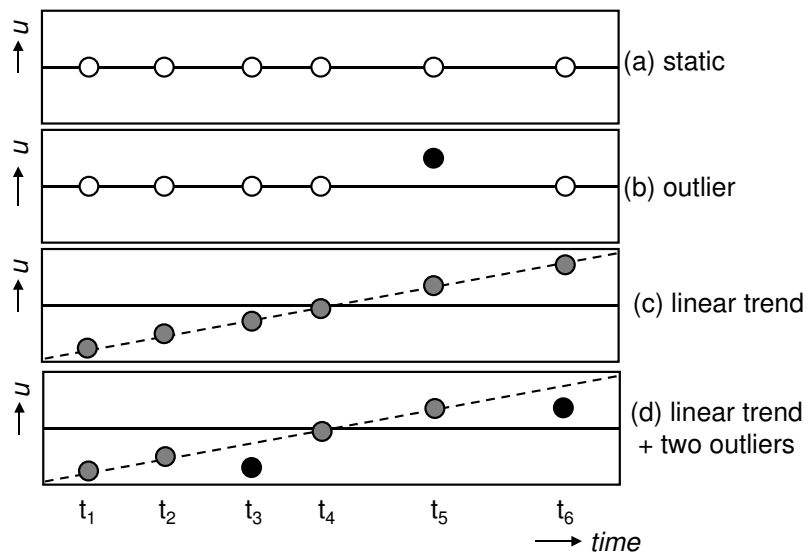

Figure 3.3: Alternative temporal sea floor characterizations.

For each parameter $u$, we consider the following extensions in time with respect to $u_{\text {ref }}$ at time $t_{\text {ref }}$ (Figure $3.3 \mathrm{~b}$ and $3.3 \mathrm{c}$ ) :

1. outlying parameter $\Delta u_{s}$ for survey $s$ :

$$
R_{s}: u(t)= \begin{cases}u_{\mathrm{ref}} & \left(\text { if } t \neq t_{s}\right) \\ u_{\mathrm{ref}}+\Delta u_{s} & \left(\text { if } t=t_{s}\right)\end{cases}
$$

2. a linear trend $\dot{u}$ of the parameter in time:

$$
R_{\mathrm{tr}}: u(t)=u_{\mathrm{ref}}+\dot{u}\left(t_{s}-t_{\mathrm{ref}}\right) .
$$

A parameter is said to be outlying for a specific survey if it significantly differs from its values for the other surveys, either due to measurement artifacts or a change in the sea floor. Also, combinations of equations (3.13) and (3.14) are allowed (Figure 3.3d), to account for nonlinearities in the trend.

A characterization of step 2 is also written as in equation (3.12), where $\mathbf{u}=\mathbf{u}(t), \mathbf{a}=\mathbf{a}(x)$ for $R_{0}$, and $\mathbf{a}=\mathbf{a}(x, t)$ for $R_{a}$. Both $\mathbf{u}$ and $\mathbf{a}$ are $N \times 1$ column vectors, with $N$ the number of parameters. The value of $N$ depends on the representation, $U \leq N \leq U S$. $N$ is at its minimum for $R_{0}$, and at its maximum if all surveys behave as outliers.

For $R^{(2)}$ and $R^{(1+2)}$, we divide $\mathbf{u}(t)$ into a set of planar parameters in $\mathbf{u}_{\mathrm{pl}}(t)$ and a set of sand wave parameters in $\mathbf{u}_{\mathrm{sw}}(t)$, which allows us to obtain different types of dynamics for the planar parameters and the sand wave parameters. Like $\mathbf{u}_{\mathrm{pl}}(t)$ and $\mathbf{u}_{\mathrm{sw}}(t)$, we divide $\mathbf{a}(x)$ into $\mathbf{a}_{\mathrm{pl}}(x)$ and $\mathbf{a}_{\mathrm{sw}}(x)$. For $R^{(1)}$ or $R^{(1+2)}$, we do not separate the parameters in $\mathbf{u}_{\mathrm{pl}}(t)$ further, as dynamics in slope imply dynamics in depth at $x=0$. 
As an example, we consider the extension of a trend in the sand wave pattern for $R^{(1+2)}$. For this extension, the morphological characterization $m_{p, s}$ of depth $\underline{d}_{p, s}$ is expressed as:

$$
m_{p, s}=\mathbf{a}_{\mathrm{ref}}^{(1+2) \mathrm{T}} \mathbf{u}_{\mathrm{ref}}^{(1+2)}+\mathbf{a}_{\mathrm{tr}, \mathrm{sw}}^{\mathrm{T}} \mathbf{u}_{\mathrm{tr}, \mathrm{sw}} .
$$

In this equation, the reference vectors $\mathbf{a}_{\text {ref }}^{(1+2) \mathrm{T}}$ and $\mathbf{u}_{\mathrm{ref}}^{(1+2) \mathrm{T}}$ are:

$$
\begin{aligned}
& \mathbf{a}_{\mathrm{ref}}^{(1+2) \mathrm{T}}=[1, x,-\cos \kappa x,-\sin \kappa x] \\
& \mathbf{u}_{\mathrm{ref}}^{(1+2) \mathrm{T}}=\left[d_{\mathrm{ref}}^{(\mathrm{b})}, \psi_{\mathrm{ref}}, A_{\mathrm{ref}}^{(\mathrm{c})}, A_{\mathrm{ref}}^{(\mathrm{s})}\right] .
\end{aligned}
$$

Further, the sand wave trend vectors $\mathbf{a}_{\mathrm{tr}, \mathrm{sw}}$ and $\mathbf{u}_{\mathrm{tr}, \mathrm{sw}}$ are:

$$
\begin{aligned}
& \mathbf{a}_{\mathrm{tr}, \mathrm{sw}}^{\mathrm{T}}=\left[-\left(t_{s}-t_{\mathrm{ref}}\right) \cos \kappa x,-\left(t_{s}-t_{\mathrm{ref}}\right) \sin \kappa x\right], \\
& \mathbf{u}_{\mathrm{tr}, \mathrm{sw}}^{\mathrm{T}}=\left[\dot{A}_{\mathrm{c}}, \dot{A}_{\mathrm{s}}\right] .
\end{aligned}
$$

\section{Vectorization of the characterization}

Let the $M \times 1$ morphology vector $\mathbf{m}$ contain the values of $m_{p, s}$, and let covariance matrix $\mathbf{C}_{d}$ contain the dispersion, as in Section 3.2.2 for the measurement error. The description of all depth measurements in any representation is then:

$$
R: \mathrm{E}\{\underline{\mathbf{d}}\}=\mathbf{m} ; \mathrm{D}\{\underline{\mathbf{d}}\}=\mathbf{C}_{d} .
$$

For vectors, the relation between the expected value and the dispersion is reformulated as $\mathrm{D}\{\underline{\mathbf{d}}\}=\mathrm{E}\left\{(\underline{\mathbf{d}}-\mathrm{E}\{\underline{\mathbf{d}}\})(\underline{\mathbf{d}}-\mathrm{E}\{\underline{\mathbf{d}}\})^{\mathrm{T}}\right\}$. The variances $\sigma_{d}^{2}$ of Section 3.2.3 are the diagonal elements of matrix $\mathbf{C}_{d}$. With the definition of $m$ in equation (3.12), we get:

$$
\mathbf{m}=\mathbf{A u}
$$

in which the $M \times N$ matrix of coefficients $\mathbf{A}$ contains the vectors $\mathbf{a}^{\mathrm{T}}$ as its rows.

\subsubsection{Morphological analysis}

\section{Overview of the analysis}

A way to select the characterization for steps 1 and 2 is the application of statistical estimation and testing theory. An overview is given here, details are treated in Appendix 3.C.

Our approach is a variant of the one taken by De Heus et al. [1994]. This approach is called the B-method of testing, developed by Baarda [1968]. We use an adapted version of the B-method, illustrated in Figure 3.4. This diagram is a more detailed view of the steps 1 and 2 of Figure 3.1.

The most likely characterization $m$ is selected by statistical extension tests, using test quotients $q_{a}$ for the alternative extensions. The extension that has the largest test quotient is added to the characterization, after which new test 


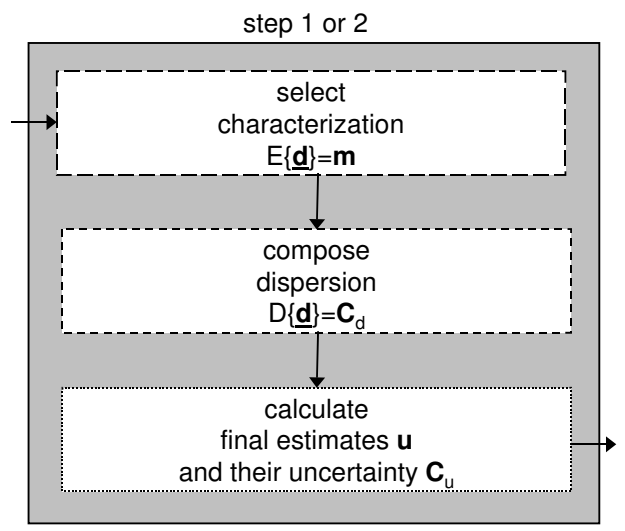

Figure 3.4: Flow diagram of our approach, modeled after the B-method of testing. This diagram is a followed in the steps 1 and 2 .

quotients are calculated. This iterative procedure continues until all remaining extensions have a test quotient smaller than one.

Hereafter, a representation is accepted if the overall test is accepted, which means that the test quotient $q^{(\mathrm{o})}$ is smaller than one. If it is larger than one, we cannot extend the characterization anymore, but relax the dispersion matrix $\mathbf{C}_{d}$ instead, by increasing its variance and covariance values. Using both the characterization and the dispersion in an estimation procedure, we obtain the final vector of estimates $\underline{\hat{\mathbf{u}}}$ and their uncertainties $\mathbf{C}_{u}$.

\section{Selection of the characterization in step 1}

In step 1 , we select the $N=U S$ significant spatial parameters for the area of the surveys, and estimate them, along with their uncertainty. All surveys must have the same spatial representation, only the parameter estimates are allowed to differ per survey.

We test the two extensions $R^{(1)}$ and $R^{(2)}$ for step 1 simultaneously, as shown in Figure 3.5. We vary the wavelength $L$ of $R^{(2)}$ over the interval $200 \mathrm{~m}<L<$ $1000 \mathrm{~m}$, to find the dominant wavelength $\underline{L}^{(\mathbf{d})}$, which we use for $R^{(2)}$. To this end, we express the test quotient of $R^{(2)}$ as:

$$
\underline{q}^{(2)}=\underline{q}^{(2)}(L)
$$

and optimize this function as:

$$
\underline{q}_{\max }^{(2)}=\max \left(\underline{q}^{(2)}(L)\right) .
$$

The dominant wavelength $\underline{L}^{(\mathrm{d})}$ is the wavelength used for $q_{\mathrm{max}}^{(2)}$. The interval is possibly further limited by the grid spacing and the length of the grid line. If 


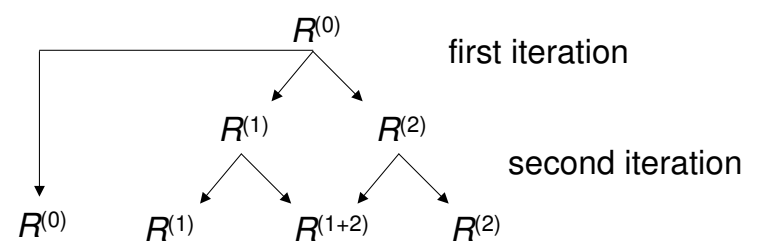

Figure 3.5: Selection of the characterization of step 1. This diagram shows the iterations in the top panel of Figure 3.4.

the maximum is found at a boundary of the interval, it is not assumed to be the dominant wavelength, and consequently no sand wave extension is accepted.

\section{Selection of the characterization in step 2}

Next, step 2 detects the behaviour of the $N \leq U S$ spatial parameters in time, and gives estimates for this behaviour, including the uncertainty of the estimates. Although the procedure is generally similar to the one for the spatial analysis of step 1, there are several differences.

The first difference is that the initial covariance matrix $\mathbf{C}_{d}^{(\mathrm{e})}$ of step 1 only contains the quantification of the errors $\mathbf{e}$, modeled by the variances $\sigma_{p, s}^{(\mathrm{e}) 2}$ and the covariance function $c_{s}^{(\mathrm{e})}\left(h_{x}\right)$, with $h_{x}$ the distance in $x$-direction. The initial covariance matrix $\underline{\mathbf{C}}_{d}^{(\mathrm{em})}$ of step 2 also includes the quantification of the morphological residuals $\mathbf{r}$, as resulting from step 1, provided that the overall test of step 1 was not accepted. The elements of the covariance matrix $\underline{\mathbf{C}}_{d}^{(\mathrm{em})}$ of step 2 are defined by the variances $\sigma_{p, s}^{(\mathrm{em}) 2}$ and the covariance function $c_{s}^{(\mathrm{em})}\left(h_{x}\right)$.

The second difference is the number of extensions. The spatial representation has only two possible extensions (Figure 3.5). For the temporal representation, $S+1$ dynamic alternatives are formulated for $R^{(0)}$ and $R^{(1)}: S$ for the outliers, and one for a trend. Likewise, $R^{(1+2)}$ and $R^{(2)}$ have $2(S+1)$ formulated alternatives: $S+1$ for the planar parameter set, and $S+1$ for the sand wave parameter set.

The third difference is that both extensions of the spatial analysis can be accepted. The temporal analysis stops if $S-1$ extensions have been made (in case of spatial representation $R^{(0)}$ or $R^{(1)}$ ) or if $S-1$ extensions have been made per parameter set (in case of spatial representation $R^{(2)}$ or $R^{(1+2)}$ ). This is to guarantee that the columns of matrix $\mathbf{A}$ remain linearly independent: a parameter without a trend can only have $S-1$ outliers, and a parameter with a trend only $S-2$. This way, the matrix $\mathbf{A}^{\mathrm{T}} \mathbf{C}_{d}^{-1} \mathbf{A}$, necessary to calculate $\mathbf{C}_{u}$ (Appendix 3.C.2), is invertible.

The fourth difference of step 2 is that we do not test all extensions simultaneously. We first test the two linear trend extensions. When no further trend 


\section{(a) selection of trends}

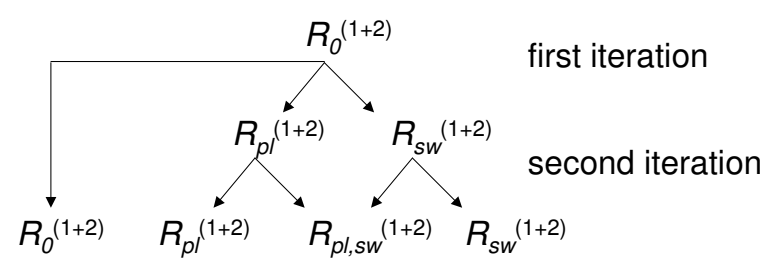

\section{(b) selection of outliers}

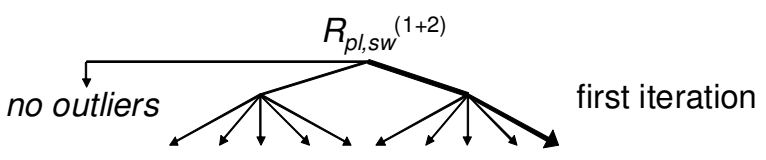

a planar outlier or a sand wave outlier

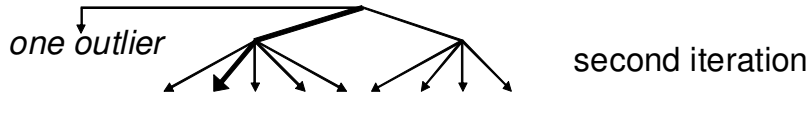

another planar or sand wave outlier

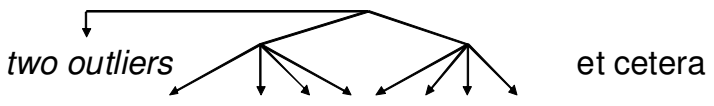

Figure 3.6: Selection of the characterization of step 2. This diagram shows the iterations in the top panel of Figure 3.4. (a) The trends are tested first, in this case for $R^{(1+2)}$; (b) the outliers are tested thereafter, in this case for $R_{\mathrm{pl}, \mathrm{sw}}^{(1+2)}$.

extensions are accepted, we continue with the extensions for outliers. This shows that we prefer to misinterpret a combination of outliers as a trend, over the opposite misinterpretation of a trend as a combination of outliers. The procedure is shown in Figure 3.6.

\subsubsection{Variance and covariance estimation}

\section{The covariance matrix after step 1}

In step 1 , the rejection of the representation by the overall test means that the components of error $\underline{e}_{p, s}$, identified in Appendix 3.A, cannot explain the residuals $\underline{r}_{p, s}^{(\mathrm{em})}$. Instead, an extension has to be made, which decreases $\underline{r}_{p, s}^{(\mathrm{em})}$, or the current covariance matrix $\mathbf{C}_{d}$ needs to be relaxed. If no further extensions can be made, the residuals are supposed to have an additional morphological 
component $r_{p, s}^{(\mathrm{m})}$, due to an incomplete characterization:

$$
\underline{r}_{p, s}^{(\mathrm{em})}=\underline{e}_{p, s}+r_{p, s}^{(\mathrm{m})} .
$$

The corresponding covariance function is:

$$
c^{(\mathrm{em})}\left(h_{x}\right)=c^{(\mathrm{e})}\left(h_{x}\right)+c^{(\mathrm{m})}\left(h_{x}\right),
$$

with variance $\sigma^{2}$ used for $c(0)$. The covariance matrices are denoted accordingly as $\mathbf{C}_{d}^{(\mathrm{e})}$ and $\mathbf{C}_{d}^{(\mathrm{em})}$. An example is given in Figure 3.7. The variance $\sigma_{s}^{(\mathrm{e}) 2}(x)$ can explain the error $\underline{e}_{s}(x)$ in Figure 3.7 a, but not the residual $\underline{r}_{s}^{(\mathrm{em})}(x)$ in Figure $3.7 \mathrm{~b}$. By increasing the variance to $\sigma_{s}^{(\mathrm{em}) 2}(x)$, using Least Squares Variance Component Estimation (LSVCE), the residual $\underline{r}_{s}^{(\mathrm{em})}(x)$ is again fully described by its variance, see Figure $3.7 \mathrm{c}$.

The elements of a covariance matrix are estimated using least squares variance component estimation (Appendix 3.D). The details for covariance matrix $\mathbf{C}_{d}^{(\mathrm{em})}$ are given in Section 3.D.4. As we have a covariance function per survey grid (Section 3.D.2), we cannot take an approach per grid line, but use cofactor matrices per grid. This generalization will be corrected by the covariance estimation per grid line of step 2.

\section{The covariance matrix after step 2}

After completing the extension process in step 2, we assume that the residuals also have a temporal component $r_{p, s}^{(\mathrm{t})}$, that accounts for the additional residuals with respect to the newly estimated characterization of step 2. We express this as:

$$
\underline{r}_{p, s}^{(\mathrm{emt})}=\underline{r}_{p, s}^{(\mathrm{em})}+r_{p, s}^{(\mathrm{t})}
$$

Therefore, we change the covariance matrix $\underline{\mathbf{C}}_{d}^{(\mathrm{em})}$ to a new matrix $\underline{\mathbf{C}}_{d}^{(\mathrm{emt})}$ that contains this temporal component, as explained in Section 3.D.5.

Subsequently, the final variances $\underline{\sigma}_{u}^{2}$ are calculated using the new matrix $\underline{\mathbf{C}}_{d}^{(\mathrm{emt})}$ (Section 3.C.2). We choose the approach of a single covariance component per grid line, just like the morphological characterization. This way, we correct the generalization of the covariance estimation per survey grid of step 1 .

\subsection{Specification of a test scenario}

\subsubsection{Specification of depth values}

The method is validated by a test scenario, based on an actual survey of an area in the Southern North Sea in 2003, using the values of the Appendices 3.C.7 and 3.C.8 for the statistical parameters $\alpha_{a}, \gamma, k_{\alpha}$ and $\lambda_{a}$. The approximate position of the area is: $51^{\circ} 58^{\prime} \mathrm{N} / 2^{\circ} 38^{\prime} \mathrm{E}$. A tidal sand wave pattern is present with a dominant wavelength $L^{(\mathrm{d})}$ of about $800 \mathrm{~m}$ (Figure 3.8a). The crest direction in Figure 3.8 corresponds to an azimuth of $121^{\circ}$ East of North. To facilitate the presentation of the results, the grid and the depth values of Figure 3.8a are 
(a)

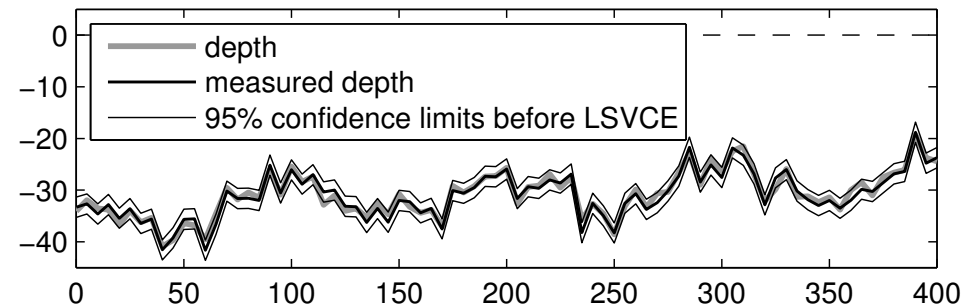

(b)

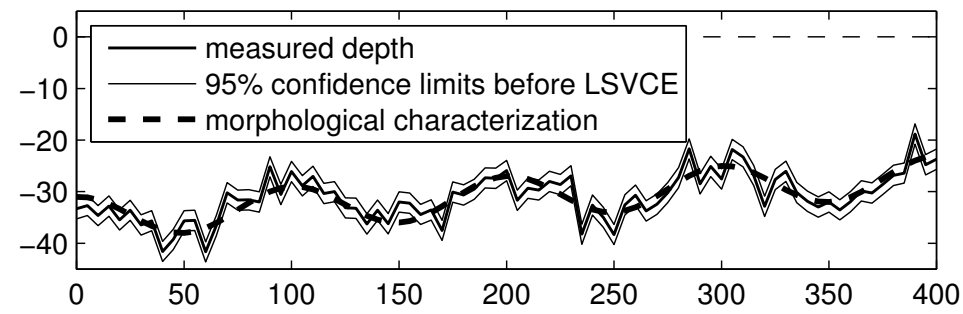

(c)

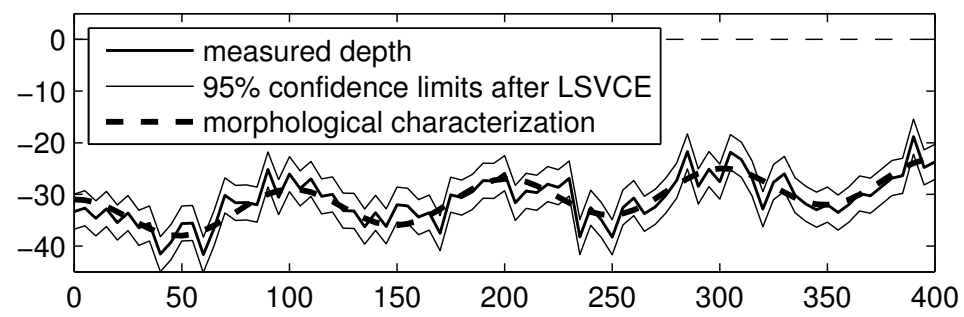

Figure 3.7: Measured depth $\underline{d}_{s}(x)$ for survey $s$ and its uncertainty at the $95 \%$ confidence interval described by $1.96 \sigma_{s}(x)$ in relation to (a) the true, unknown depth $d_{s}(x)$ and the original variance $\sigma_{s}^{(\mathrm{e}) 2}(x)$ for the measurement error $\underline{e}_{s}(x)$; (b) morphological characterization $m_{s}(x)$ and the original variance $\sigma_{s}^{(\mathrm{e}) 2}(x)$; (c) morphological characterization $m_{s}(x)$ and the increased variance $\sigma_{s}^{(\mathrm{em}) 2}(x)$ for the residual $\underline{r}_{s}^{(\mathrm{em})}(x)$.

redrawn as a contour plot, in Figure 3.9. The graphs $3.8 \mathrm{~b}-\mathrm{d}$ will be discussed in Section 3.4.1.

To allow for a critical analysis of the performance of the proposed method, on purpose an area is selected for which the sand wave pattern is rather irregular, and the dynamics are simulated. There is a bifurcation in the crest, behind which the pattern becomes very irregular.

As described in Section 3.2.2, the input consists of five quantities. The fixed positions $\mathbf{x}_{p}$ (quantity 1) are given at a grid with a spacing of $50 \mathrm{~m}$. This is dense enough to capture sand wave dynamics for the given wavelength $L$. The orientation of the $x$ - and the $y$-axis are perpendicular to and parallel to the crest 
(a) depth

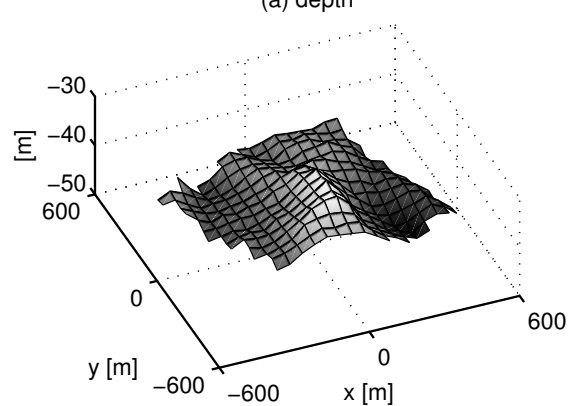

(c) $95 \%$ confidence level

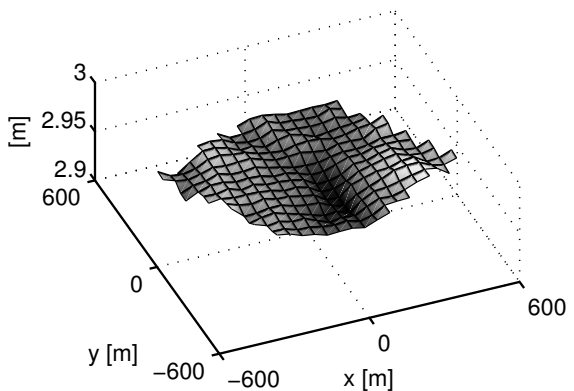

(b) characterization

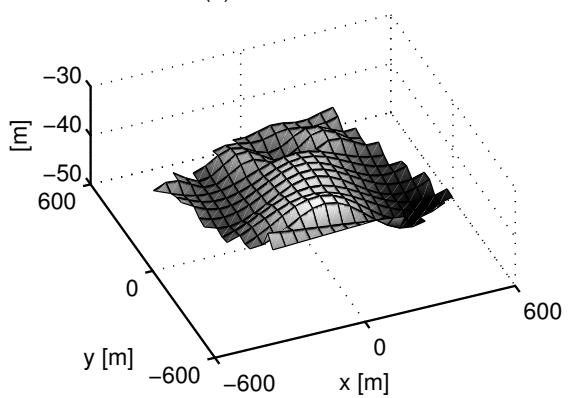

(d) residuals

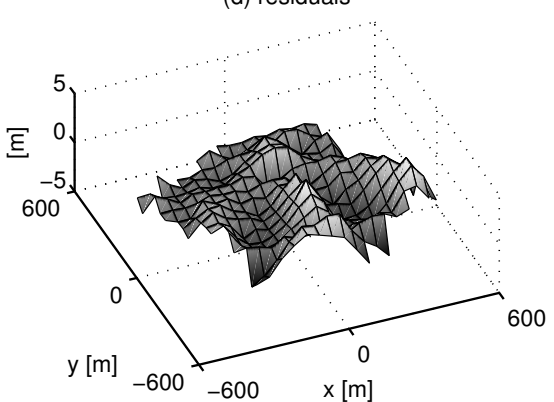

Figure 3.8: Overview of the example: (a) gridded depth values $\underline{d}$ below Lowest Astronomical Tide; (b) characterization $\underline{\hat{m}}$ of the gridded depth values, after the spatial testing and estimation process; (c) dispersion of the gridded depth values at the $95 \%$ confidence level, $1.96 \underline{\sigma}^{(\mathrm{em})}$, after the estimation of spatial variance and covariance components; (d) residuals $\underline{\hat{r}}$ for the gridded depth values. All values are averages over the six surveys. Note that all vertical axes have been exaggerated with respect to the horizontal axes. The $x$-direction has the highest variability, and is also the direction of the survey tracks. The grid lines are the lines in this direction.

direction, respectively. We surveyed this area six times in twelve years: $\mathbf{t}=[0$ $\left.\begin{array}{lllll}4 & 9 & 11 & 12\end{array}\right]^{\mathrm{T}}$ (quantity 2), which is realistic for this part of the Netherlands Continental Shelf, according to the present resurvey policy of the Netherlands Hydrographic Service.

To obtain depth values $\underline{d}_{p, s}$ (quantity 3 ), we assign the 2003 values to all the surveys $s$, and add simulated noise with a normal distribution to each survey. This noise represents a depth uncertainty of $0.74 \mathrm{~m}$ at the $95 \%$ confidence level, except for the first survey, where we assume the uncertainty to be $1.38 \mathrm{~m}$. The higher data uncertainty might be due to bad weather during the survey, a larger track spacing, or less accurate equipment. The above uncertainties correspond to the IHO S44, order 1 and order 2 standards respectively, for a depth of $40 \mathrm{~m}$ [International Hydrographic Organization, 2008a]. 


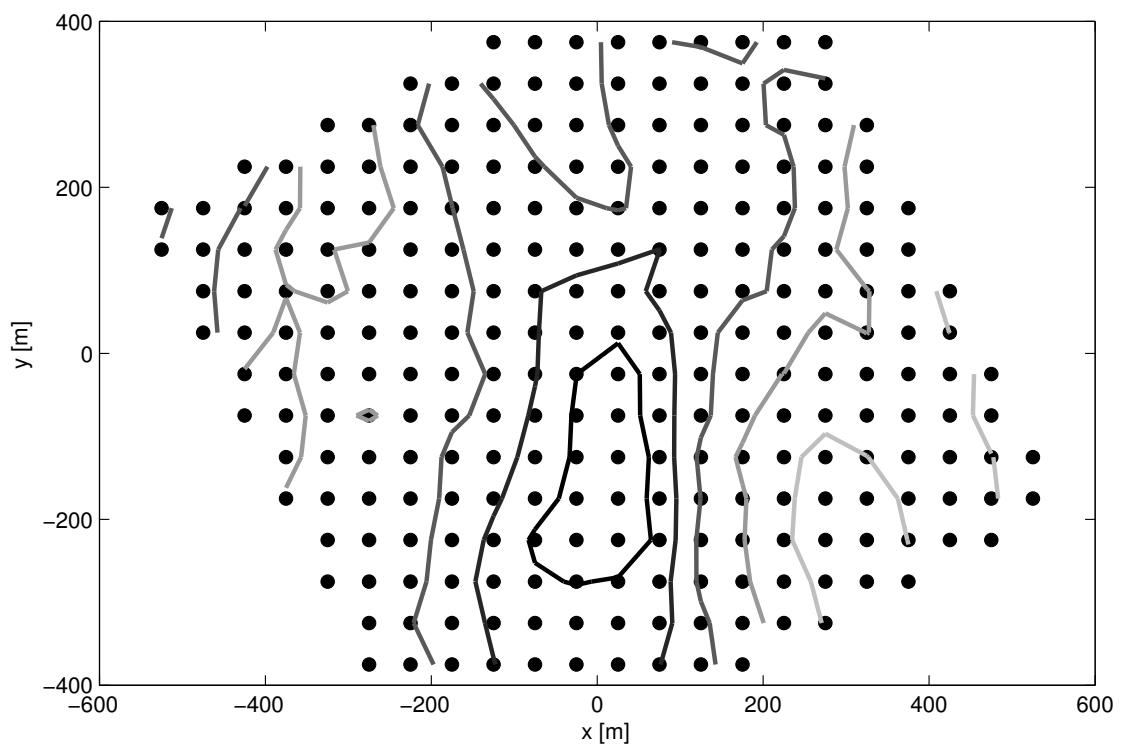

Figure 3.9: The used grid (black dots) and depth contour lines, drawn at $2 \mathrm{~m}$ intervals. The darkest line is the shallowest, at $35 \mathrm{~m}$, and the lightest line the deepest, at $43 \mathrm{~m}$.

Next, we artificially impose several types of dynamics to the $2 \mathrm{D}$ grids of surveys. To this end, we consider the simulated sea floor data of each survey as a superposition of a sloping plane and a residual pattern. The plane is fitted through the data by a least-squares procedure, and the residual pattern represents the sand wave. This allows us to assign simulated pattern dynamics, independent of simulated bed level dynamics. After the addition of dynamics, the sea floor is reconstructed by the summation of the residuals and the sloping planes, for each survey.

We impose the following dynamics:

1. Every depth of the third survey is reduced by $\Delta d=-1 \mathrm{~m}$, possibly because of a systematic error in the acquisition process.

2. The magnitude of the sand wave pattern is reduced to a factor 0.5 for the fifth survey, to incorporate the eroding effect of a storm on the sand wave amplitude $A$.

3. We let the pattern migrate with a rate of $\dot{\xi}^{\prime}=8 \mathrm{~m} / \mathrm{yr}$ in the positive $x$ direction. For each survey, the shifted pattern is interpolated to the grid using piecewise cubic hermite polynomials in the $x$-direction [Fritsch and Carlson, 1980]. 


\subsubsection{Specification of uncertainty}

We assume for the simulated surveys that the depth uncertainty is caused by the vertical errors $\underline{e}_{p, s}^{(\mathrm{v})}$ in the depth measurement process only, see Appendix 3.A. The depth errors due to positioning uncertainty $\underline{e}_{p, s}^{(\mathrm{h})}$ are assumed negligible, because of the relatively smooth nature of the sea floor. It is also assumed that the original data were that dense that no interpolation is needed. As a consequence, we consider the interpolation error $\underline{e}_{p, s}^{(\mathrm{k})}$ to be zero. For many surveys, one or both of these assumptions cannot be made, and a more complicated error modeling might be necessary.

The variances (quantity 4) of the depth measurement errors $\underline{e}_{p, s}^{(\mathrm{v})}$ are modeled according to the IHO S44 standards [International Hydrographic Organization, 2008a], which are depth dependent. Just like the simulation of measurement noise, we assume order 1 uncertainty, except for the first survey, where we assume order 2 uncertainty. These standards define depth accuracy at the $95 \%$ level as:

$$
1.96 \sigma_{p, s}^{(\mathrm{e})}=\sqrt{a^{2}+\left(b d_{p, s}\right)^{2}}
$$

with depth $d_{p, s}$, and parameters $a$ and $b$, which are $0.5 \mathrm{~m}$ and 0.013 respectively for order 1 , and $1.0 \mathrm{~m}$ and 0.026 for order 2 .

The most important contribution to the error $\underline{e}_{p, s}^{(\mathrm{v})}$ of depth measurement in the Southern North Sea outside the territorial sea is the water level reduction. It is common to reduce the depth measurements to a low water surface, nowadays Lowest Astronomical Tide (LAT). Consequently, the actual water level above this reduction surface is determined. The water level values, above the reduction surface, change relatively slowly, and are updated once every ten minutes. The error covariance $c_{p, q, s}^{(\mathrm{e})}$ (quantity 5 ) is therefore modeled as an anisotropic Gaussian function, with its highest covariance in the track direction $x$ :

$$
c_{s}^{(\mathrm{e})}\left(h_{x}\right)=c_{0}^{(\mathrm{e})} \exp \left(-\left(h_{x} / h_{x}^{(\mathrm{i}, \mathrm{e})}\right)^{2}\right) .
$$

A similar Gaussian function is used in the perpendicular direction $y$. In our scenario, the track direction coincides with the $x$-direction, perpendicular to the crest direction. The inflection points of the Gaussian function are at $h_{x}^{(\mathrm{i}, \mathrm{e})}$ $=2500 \mathrm{~m}$ and $h_{y}^{(\mathrm{i}, \mathrm{e})}=50 \mathrm{~m}$ in and across track direction, respectively. The reduction error is assumed to be $0.2 \mathrm{~m}$ at the $95 \%$ confidence level, equivalent to a variance of $0.01 \mathrm{~m}^{2}$. This value is assigned to $c_{0}^{(\mathrm{e})}$.

\subsection{Analysis results of a dynamic sea floor}

The simulated dynamics of Section 3.3 are analyzed in the two steps of Section 3.2. The results are presented in the Sections 3.4.1 and 3.4.2, respectively.

\subsubsection{Results of step 1, the spatial analysis}

At grid nodes larger than $y=125 \mathrm{~m}$, the crest bifurcates into two less pronounced crests. The test quotients $\underline{q}$ of step 1, given in Figure 3.10, show that the 
dominant wavelengths $\underline{L}^{(\mathrm{d})}$ of the grid lines are between $600 \mathrm{~m}$ and $800 \mathrm{~m}$ until the bifurcation. Behind the bifurcation, the wavelengths are smaller. The dominant wavelength is most prominent at the grid lines with a negative $y$ coordinate, as the values of the test quotients show. The short grid lines at $y=-375 \mathrm{~m}$ and $y=-325 \mathrm{~m}$ are too short to find the dominant wavelength. Figure $3.10 \mathrm{~b}$ shows that the sand wave extension (with test quotient $\underline{q}_{\max }^{(2)}$ ) usually is the first one to be accepted, which is usually followed by the slope extension (with test quotient $\underline{q}^{(1)}$ ). A slope extension is accepted for every grid line. The resulting sea floor characterization $\underline{\hat{m}}$ is shown in Figure $3.8 \mathrm{~b}$.

The difference with the depth grid is the residual variation $\underline{\hat{r}}$, shown in Figure 3.8d. Large residuals are visible at the crest, in the center of the area, because of crest-trough asymmetry. Large deviations are also visible at the shorter lines, that are too short to detect the dominant wavelength. Clearly, a rhythmic pattern is present at half the averaged dominant wavelength. This illustrates the influence of asymmetry of the sand waves on the residuals.

Figure 3.10c shows that the extensions are not yet sufficient to accept the representation, as the overall tests $\underline{q}^{(\mathrm{o}, \mathrm{m})}$ are still larger than one, after the final iteration. We conclude that additional sea floor variation is present, to be modeled by a variance factor $\underline{\hat{\sigma}}_{s}^{(\mathrm{m}) 2}$ and a covariance factor $\underline{c}_{0, s}^{(\mathrm{em})}$ per survey. The variance factor obtained for the first, less accurately measured, survey is $3 \mathrm{~m}^{2}$. For the fifth survey, for which the pattern magnitude is smaller, the variance factor is $0.5 \mathrm{~m}^{2}$. The other surveys have variance factors of $2.0 \mathrm{~m}^{2}$ to $2.5 \mathrm{~m}^{2}$.

The covariance functions for the estimation of the covariance factors are shown in Figure 3.11. The reduction of the pattern in the fifth survey is clearly visible in Figure 3.11e. The other five figures are very similar to each other. The resulting variance is given as variation at the $95 \%$ confidence level $1.96 \underline{\sigma}^{(\mathrm{em})}$ in figure 3.8c. The constant wavelength of the covariance functions $\underline{L}^{(\mathrm{em})}$ is $388 \mathrm{~m}$, which is about half the dominant wavelength of the sand wave representations indeed (Figure 3.10). For the first until the fifth survey, the inflection points $h_{x, s}^{(\mathrm{i}, \mathrm{em})}$ are placed at the maximum distance of $550 \mathrm{~m}$. The sixth survey has a shorter inflection distance of $527 \mathrm{~m}$, due to the effect of the noise that we applied.

\subsubsection{Results of step 2, the temporal analysis}

In step 2, the procedure accepts many dynamic extensions, as shown by the test quotients $q$ in Figure 3.12. Table 3.1 gives a overview whether the accepted dynamics match the dynamics specified in Section 3.3.1.

For most of the grid lines, the migration of the sand wave turns out to be the easiest detection, as this extension has, in general, the largest test quotients $\underline{q}_{\mathrm{tr}, \mathrm{sw}}$ (Figure 3.12b). Moreover, it is detected in the first iteration for most of the grid lines (Figure 3.12a). Hereafter, the two modeled outliers are detected, where either the planar depth reduction or the reduction in magnitude of the sand wave pattern have a larger test quotient. For some grid lines, other extensions are incorrectly accepted, or the modeled extensions remain incorrectly undetected. 

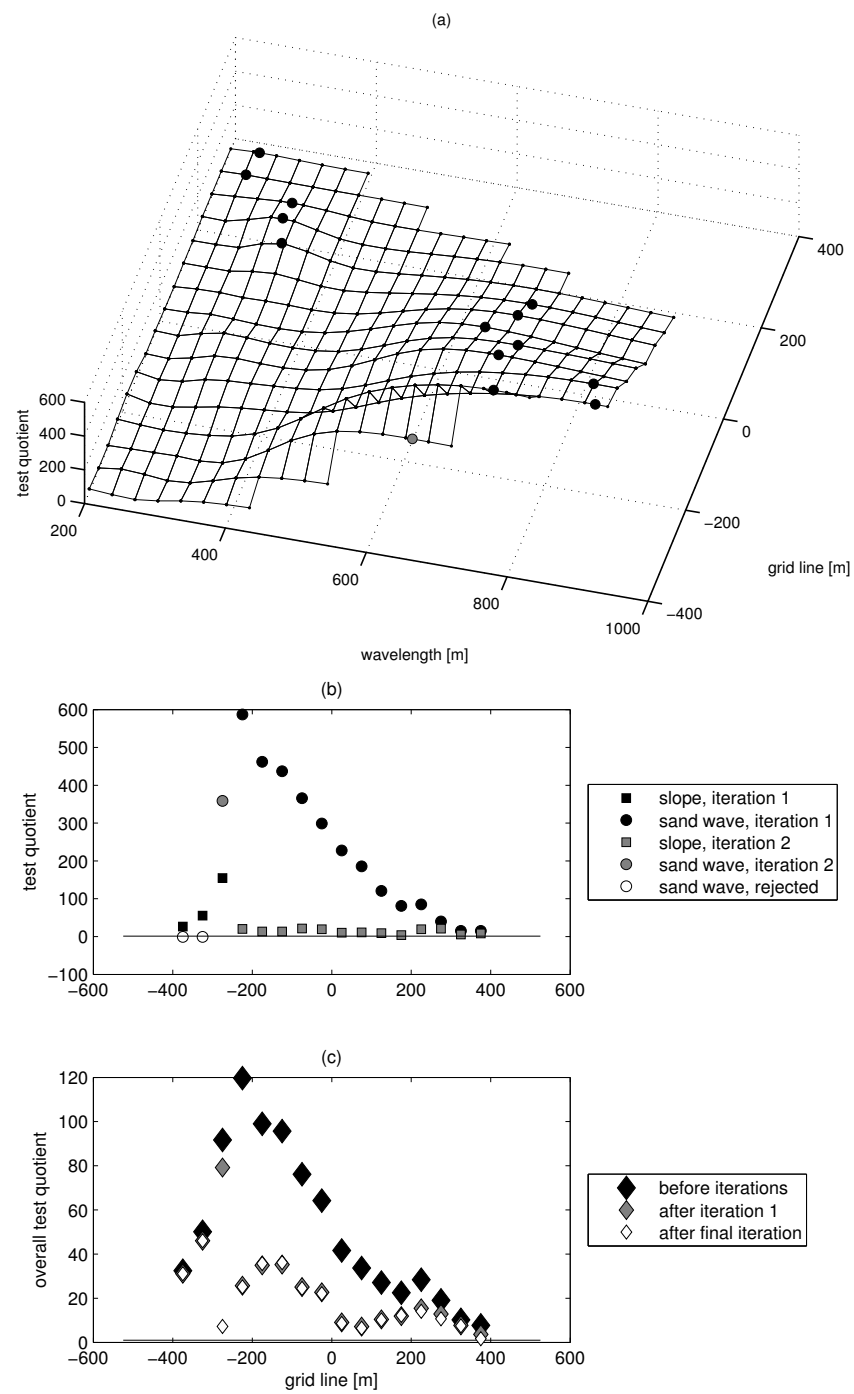

Figure 3.10: Test results for each grid line, for step 1. (a) spatial test quotients $\underline{q}^{(2)}(L)$ and $\underline{q}_{\max }^{(2)}$ (circles) for the sand wave extension; (b) spatial test quotients $\underline{q}^{(1)}$ (squares) and $\underline{q}_{\max }^{(2)}$ (circles) for the slope and the sand wave extension respectively; (c) overall test quotients $\underline{q}^{(\mathrm{o}, \mathrm{m})}$ (diamonds). The circles of Graph (a) are the maximum values $\underline{q}_{\text {max }}^{(2)}$, that correspond to the dominant wavelength $\underline{L}^{(\mathrm{d})}$ of a grid line. A black symbol in Graph (a) and (b) indicate a test quotient during the first iteration, and a grey symbol a test quotient during the second iteration. Shade and size of the diamonds in Graph (c) decrease for every next iteration. 


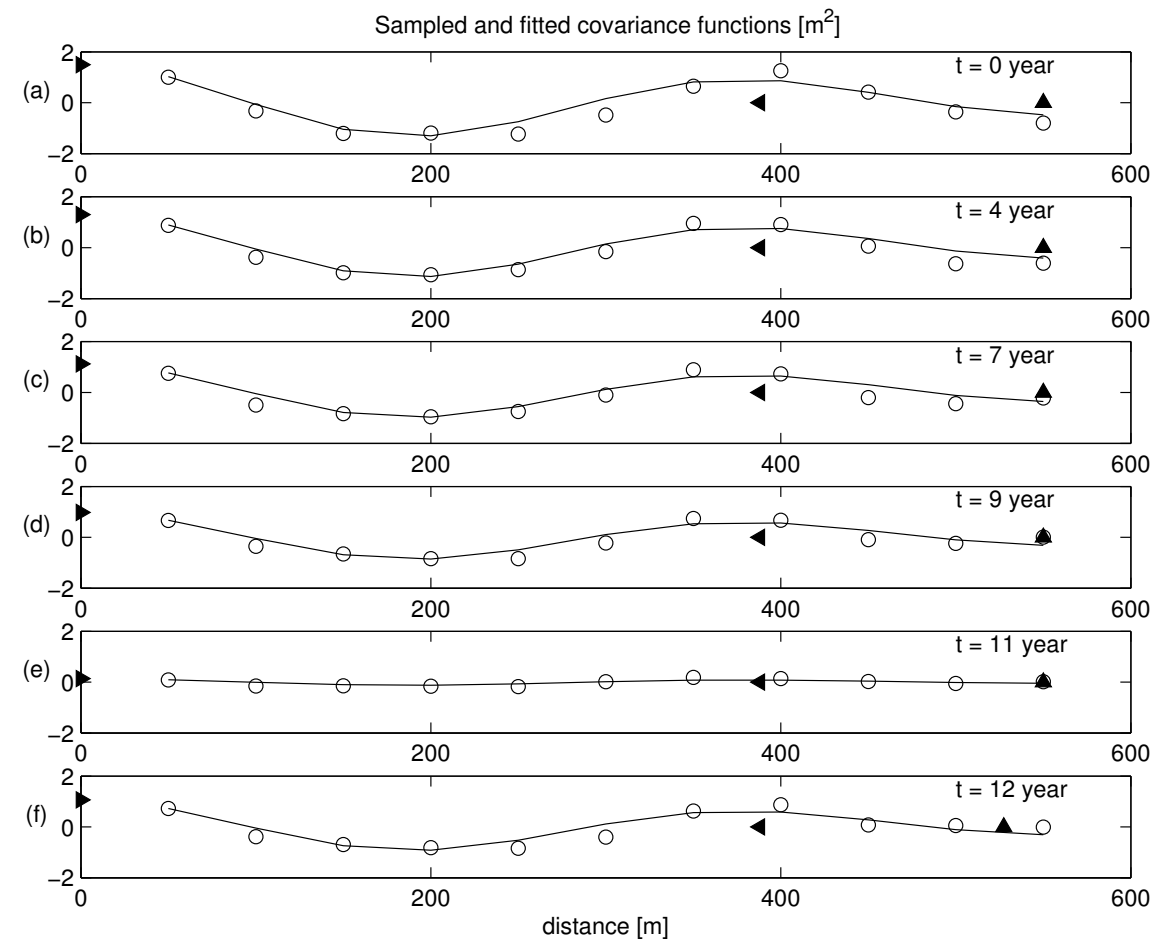

Figure 3.11: Covariance functions $\underline{c}_{s}^{(\mathrm{em})}\left(h_{x}\right)$ that describe the residuals after step 1 , for each survey $s$. Graph (a) to (f) correspond to survey one to six, respectively. The circles are the discrete covariance values, and the line is the positive-definite function $\underline{c}_{s}^{(\mathrm{em})}\left(h_{x}\right)$ that is estimated based on them. The right pointing triangle shows the covariance at a distance approximating zero $\underline{c}_{0, s}^{(\mathrm{em})}$, the up-pointing triangle the Gaussian inflection point $h_{x, s}^{(\mathrm{i}, \mathrm{em})}$, and the left-pointing triangle the wavelength $\underline{L}^{(\mathrm{em})}$.

Table 3.1: Overview of the accepted dynamics, as a percentage of the number of grid lines

\begin{tabular}{|c|c|c|}
\hline type of dynamics & specified & accepted \\
\hline outlying plane, survey 3 & yes & $94 \%$ \\
\hline trend in sand wave & yes & $71 \%$ \\
\hline outlying sand wave, survey 5 & yes & $64 \%$ \\
\hline trend in plane & no & $44 \%$ \\
\hline outlying plane, survey 4 & no & $6 \%$ \\
\hline outlying plane, survey 5 & no & $6 \%$ \\
\hline outlying plane, survey 6 & no & $6 \%$ \\
\hline
\end{tabular}



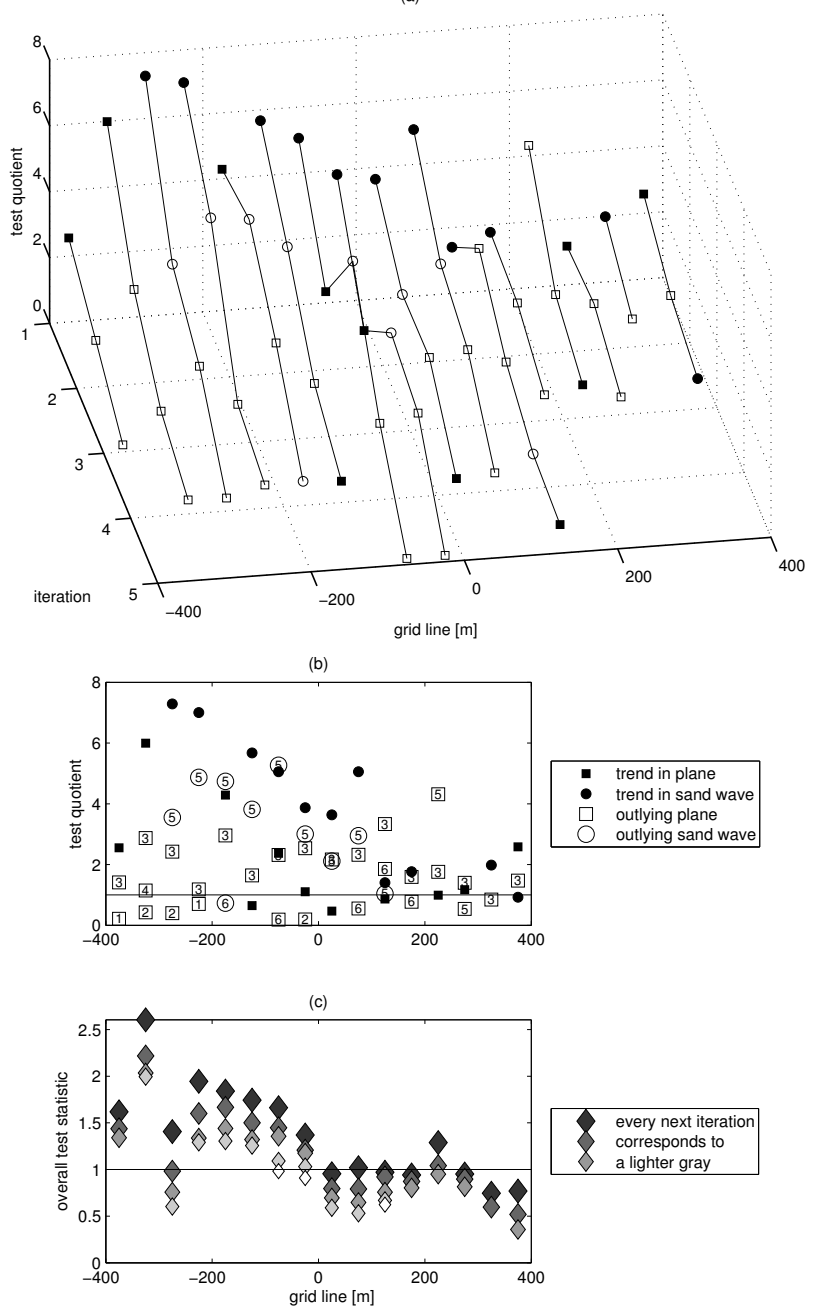

Figure 3.12: Test results for each grid line, for step 2, for the accepted extensions and the extension with the largest remaining test quotient. (a) temporal test quotients $\underline{q}_{a}$ per iteration; (b) temporal test quotients $\underline{q}_{a}$; (c) overall test quotients $\underline{q}^{(\mathrm{o}, \mathrm{t})}$. A black symbol in Graph (a) and (b) indicate a test quotient for a trend, and a white symbol a test quotient for an outlier. Test quotients for planar extensions are given by squares, and test quotients for sand wave extensions by circles. The numbers in Graph (b) are the survey numbers $s$ for which the outlier is detected. Shade and size of the diamonds in Graph (c) decrease for every next iteration. 
Figure $3.12 \mathrm{c}$ shows that the overall test quotient $q^{(\mathrm{o}, \mathrm{t})}$ usually becomes smaller than one, several iterations before all extensions were made. This means that the final variances $\underline{\sigma}_{p, s}^{(\mathrm{emt}) 2}$ of the parameters are reduced with respect to the variances $\underline{\sigma}_{p, s}^{(\mathrm{em}) 2}$ after step 1 . This is as expected, as in our test scenario the residuals are highly correlated between the surveys, as we did not introduce any small-scale dynamics.

The grid lines at $y=-375 \mathrm{~m}$ and $y=-325 \mathrm{~m}$, for which no dominant wavelength could be found, show large overall test quotients. This is because residuals are evidently larger in the absence of sand wave parameters. As a result, the variances of the parameters are larger.

The results of the morphological analysis are presented in the Figures 3.13 to 3.16 , with planar results on the left side and sand wave results on the right side of the figures. The first survey is used as a reference, presented in the Figures 3.13 and 3.14, for the dynamic parameters. Those dynamic parameters are presented in the Figures 3.15 and 3.16, and for the grid line $y=25 \mathrm{~m}$ in Table 3.2. The dominant wavelengths $\underline{L}^{(\mathrm{d})}$ are given in Figure 3.14a. These wavelengths correspond to the detected wavelengths that are shown in Figure 3.10a.
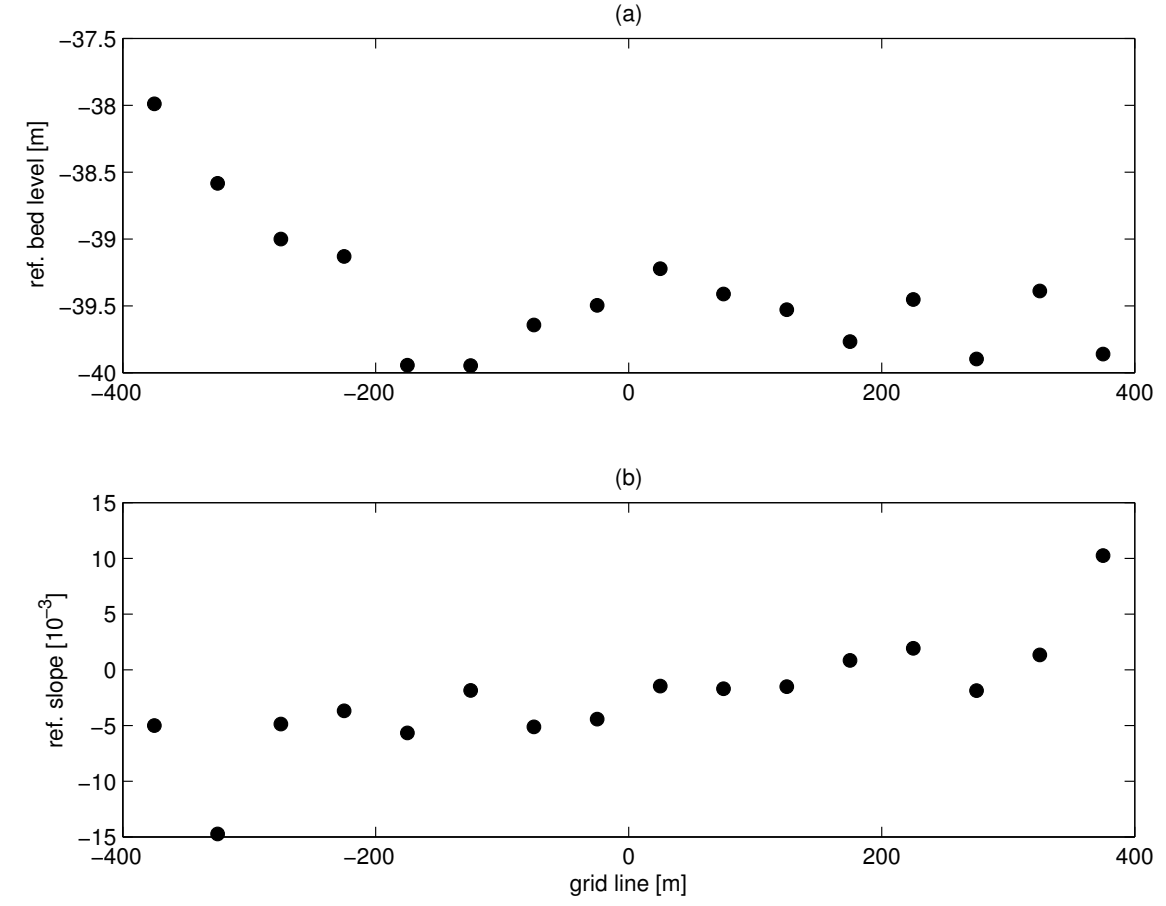

Figure 3.13: Reference values for the parameters, equal to the values of the first survey. (a) for the planar parameter bed level $-\underline{d}_{\mathrm{ref}}^{(\text {b) }}$; (b) for the planar parameter slope $-\underline{\psi}_{\text {ref }}$. 
The dominant wavelength is more or less constant over the grid lines, except for the grid lines $y \geq 175 \mathrm{~m}$, where the depth values become less structured. Although the wavelengths decrease sharply, the reference amplitudes $\underline{A}_{\text {ref }}$ only gradually become smaller. This is because the 600 to $800 \mathrm{~m}$ wavelength gradually becomes less prominent, and at the same rate the half wavelength becomes more prominent (Figure 3.10a). At the grid line $y=175 \mathrm{~m}$, the half wavelength suddenly becomes dominant. As the amplitude is a parameter, it can change gradually. The sudden change in wavelength also affects the position of the crest (Figure 3.14c).

We make several observations from the Figures 3.15 and 3.16, in combination with Figure 3.12. Firstly, we see that the simulated dynamics were found for a majority of the grid lines, and that the parameters for all incorrectly detected dynamics are small in comparison to their $95 \%$ confidence intervals. Also note that a detected trend in either bed level $-\underline{d}^{(\mathrm{b})}$ or slope $\psi$ also results in a detection of a change in the other. This is also valid for the outliers, and the

(a)

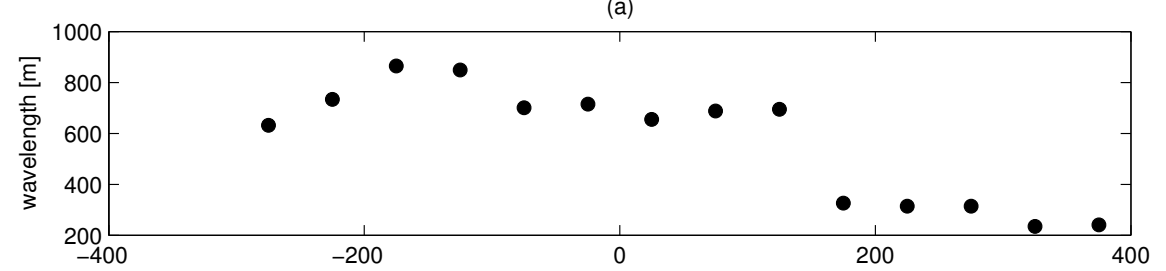

(b)

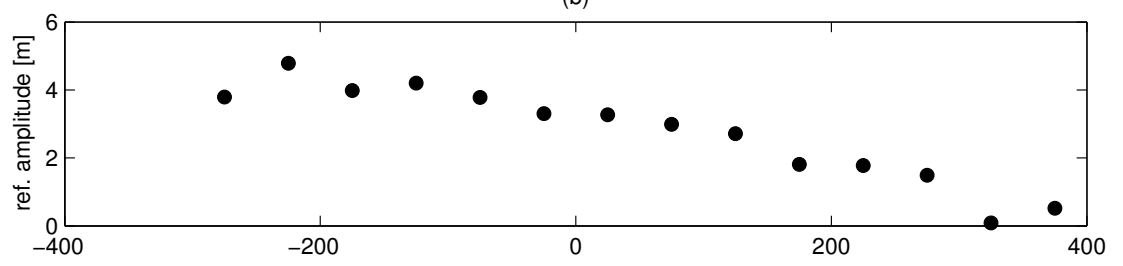

(c)

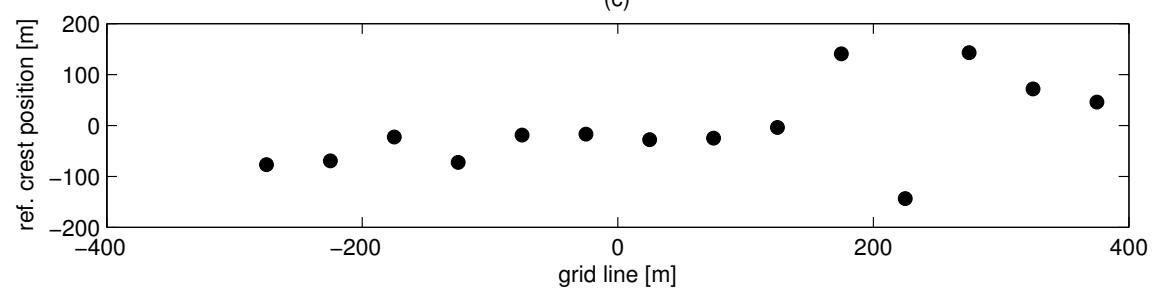

Figure 3.14: Reference values for the parameters, equal to the values of the first survey (continued). (a) for dominant wavelength $\underline{L}^{(\mathrm{d})}$ for all surveys (also shown in Figure 3.10a); (b) for sand wave parameter amplitude $\underline{A}_{\text {ref }}$; and (c): for sand wave parameter crest position $\underline{\xi}_{\text {ref }}$. 

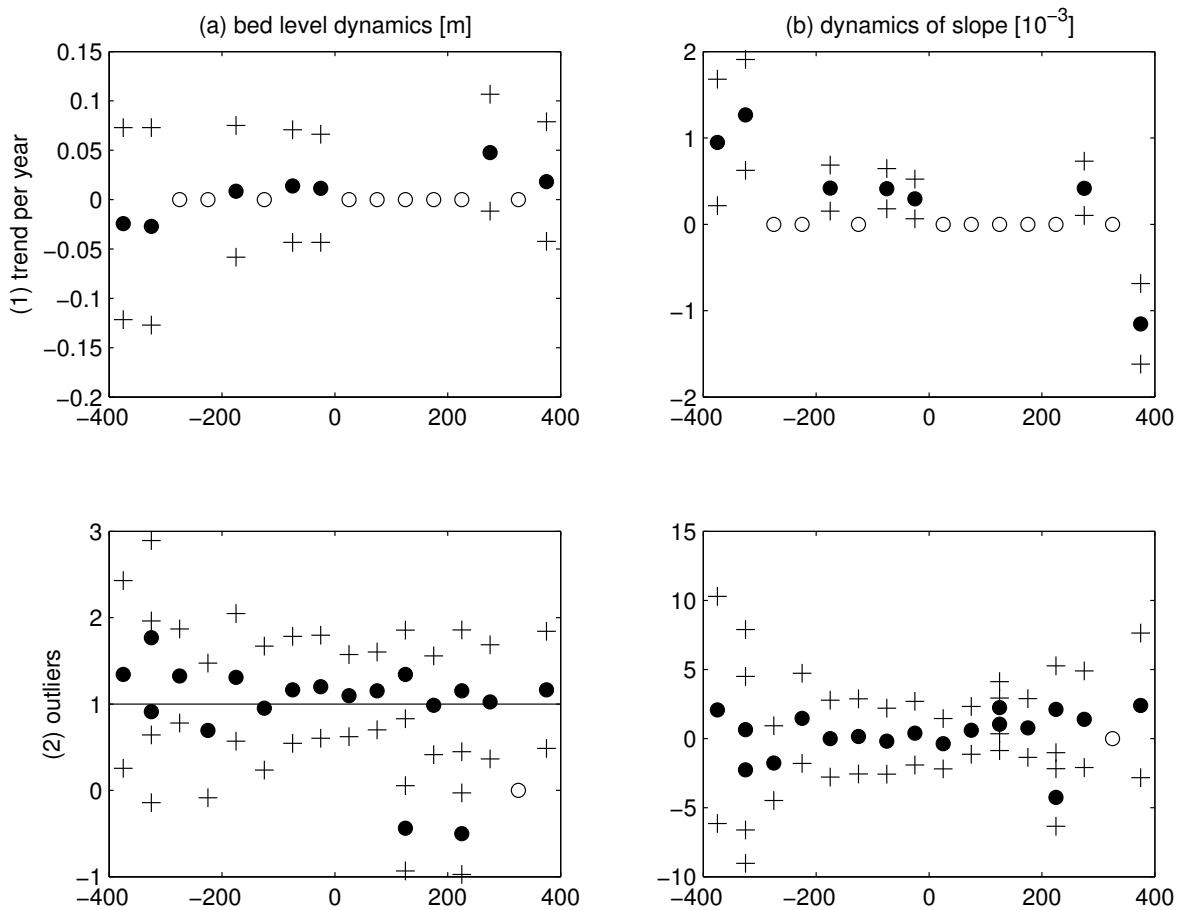

Figure 3.15: Results for the temporal analysis. $x$-axes: grid line $[\mathrm{m}] ; y$-axes: results as indicated per row and column of graphs. Columns (a) and (b): planar parameters $-\underline{d}^{(\mathrm{b})}$ and $-\underline{\psi}$. The trends are given in row (1), and the outliers in row (2). White circles indicate the absence of dynamics, and black circles the presence of dynamics, including the uncertainty at the $95 \%$ confidence level, indicated by plus symbols. The slope $-\underline{\psi}$ is expressed as vertical change in $\mathrm{m}$ per horizontal $\mathrm{m}$, and therefore dimensionless. The horizontal lines indicate the specified dynamics of the test scenario. The results for the grid line $y=25 \mathrm{~m}$ are also given in Table 3.2.

two sand wave parameters $\underline{A}^{\prime}$ and $\underline{\xi}^{\prime}$. The reason is that the extensions all consist of two parameters, that are mutually correlated.

For a second observation, we look at row (1), containing the trends. The presence of sand wave migration is sometimes misinterpreted as a trend in the planar parameters (e.g. $y=-175 \mathrm{~m}$ ), sometimes an erroneous planar trend is found in addition to the sand wave trend (e.g. $y=-25 \mathrm{~m}$ ), and sometimes no trend is found (e.g. $y=225 \mathrm{~m}$ ). For the grid lines at $y=-375 \mathrm{~m}$ and $y=-325 \mathrm{~m}$, a planar trend was detected because no sand wave parameters are present. The planar misdetections happen because extensions for the plane are correlated with those for the sand wave. The two types of extensions are only independent if the grid line length is an exact multiple of the wavelength. This misinterpretation 

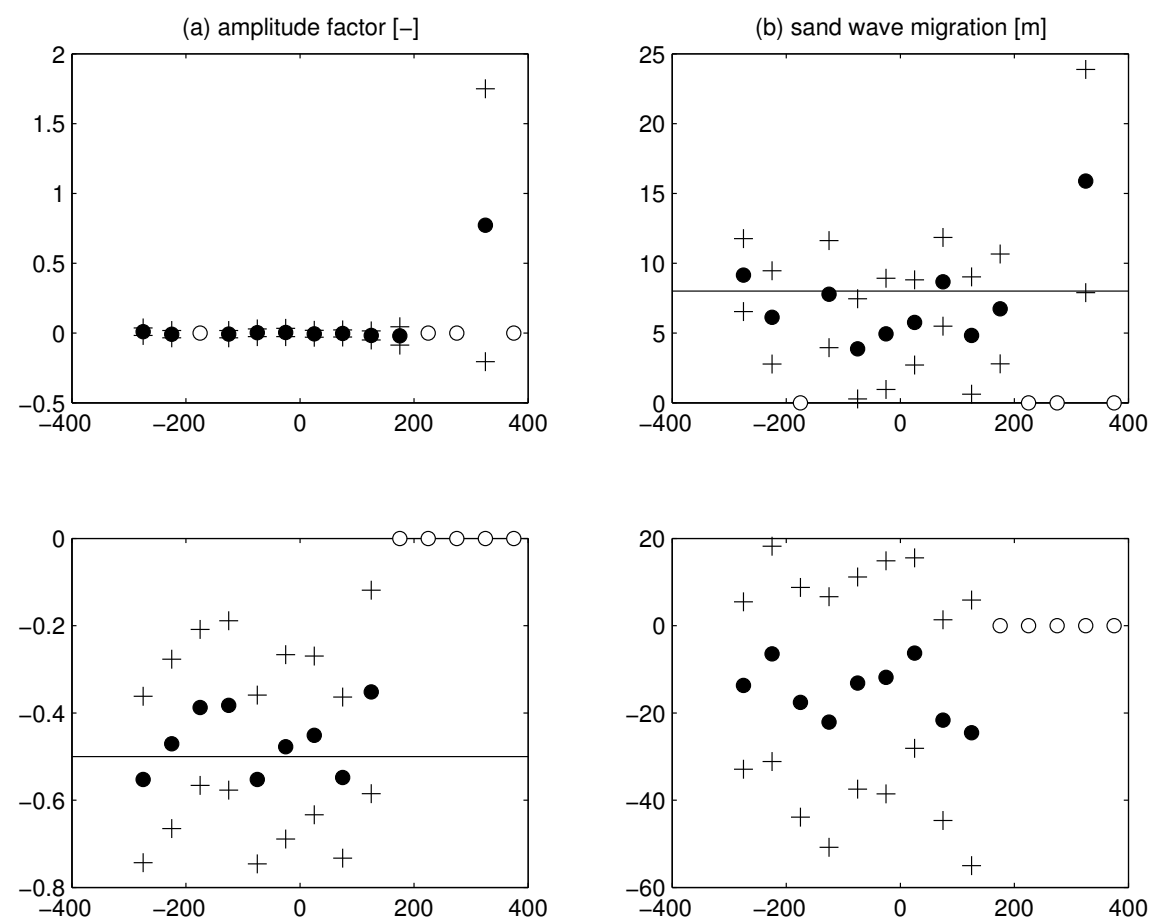

Figure 3.16: Results for the temporal analysis (continued). Columns (a) and (b): sand wave parameters $\underline{A}^{\prime}$ and $\underline{\xi}^{\prime}$.

is especially likely in the case of a small number of sand waves, as in this test scenario.

Thirdly, we look at the outliers, in row (2). The planar outlier is estimated correctly for almost every grid line, see the bottom graph of column (a). Their confidence intervals are very different, though. It is clear that the least accurate estimations are those from the grid lines where no sand wave was accepted, because the magnitude of their residuals are larger. Accepting a representation with a sand wave therefore also helps us to estimate planar parameters more accurately.

The reduction in magnitude of the pattern was only found for those grid lines where this pattern is still fairly regular, see the bottom graph of column (c). For grid lines $y \geq 175 \mathrm{~m}$, the pattern is too unstructured to detect amplitude change.

A final observation is that there is a clear relation between the reference amplitude of the estimated sand wave on one hand, and the amplitude factor and migration rate on the other hand. The small amplitudes of grid lines $y \geq 175$ $m$ mean that amplitude change and migration are hard to estimate, as the large confidence intervals for those grid lines in the graphs on row (1) of columns (c) 
Table 3.2: Results for grid line $y=25 \mathrm{~m}$, where the dominant wavelength $\underline{L}^{(\mathrm{d})}$ is 655 $\mathrm{m}$. The results (also shown in the Figures 3.13 to 3.16 ) include the $95 \%$ confidence limits. The slope is expressed as vertical change in $\mathrm{m}$ per horizontal $\mathrm{m}$, and therefore dimensionless.

\begin{tabular}{|c|c|c|}
\hline name: & symbol $=$ & estimate \pm uncertainty \\
\hline reference depth: & $\underline{d}_{\mathrm{ref}}^{(\mathrm{b})}=$ & $39.22 \mathrm{~m} \pm 0.17 \mathrm{~m}$ \\
\hline reference slope: & $\psi_{\text {ref }}=$ & $1.5 \cdot 10^{-3} \pm 0.6 \cdot 10^{-3}$ \\
\hline reference amplitude: & $\underline{\bar{A}}_{\mathrm{ref}}=$ & $3.27 \mathrm{~m} \pm 0.33 \mathrm{~m}$ \\
\hline reference crest position: & & $-28 \mathrm{~m} \pm 12 \mathrm{~m}$ \\
\hline depth change for $s=3$ : & $\Delta \underline{d}_{3}^{(\mathrm{b})}=$ & $-1.10 \mathrm{~m} \pm 0.48 \mathrm{~m}$ \\
\hline slope change for $s=3$ : & $\Delta \psi_{3}=$ & $0.4 \cdot 10^{-3} \pm 1.8 \cdot 10^{-3}$ \\
\hline amplitude factor for $s=5$ : & ${\underline{A_{5}^{\prime}}}^{\prime}=$ & $-0.45 \pm 0.18$ \\
\hline migration for $s=5$ : & & $-6 \mathrm{~m} \pm 22 \mathrm{~m}$ \\
\hline amplitude growth rate: & $\underline{\dot{A}}^{\prime}=$ & $-0.01 \mathrm{yr}^{-1} \pm 0.03 \mathrm{yr}^{-1}$ \\
\hline migration rate: & & $6 \mathrm{~m} / \mathrm{yr} \pm 3 \mathrm{~m} / \mathrm{yr}$ \\
\hline
\end{tabular}

and (d) show. For $y=325 \mathrm{~m}$, it results in a spurious estimation of migration, and for $y=375 \mathrm{~m}$, a spurious trend in slope was detected. In spite of the listed occasional spurious results, The Figures 3.15 and 3.16 illustrates that the general achievement of the designed method for this challenging morphological pattern is good.

\subsection{Discussion}

\subsubsection{Input}

As noted in Section 3.2.2, application of deformation analysis to the sea floor imposes some requirements on the input. Firstly, the method uses depth values and their uncertainties at equal positions for several surveys. An equal number of grid nodes and equal nodal positions for all surveys are necessary, because a varying spatial distribution of nodes over time could introduce false detections of sea floor dynamics.

Secondly, the user must specify levels of significance as input. Besides the probability of accepting an extension, they also express the relative importance of detection of the defined extensions. Therefore, those levels of significance can vary with the goal of an analysis.

Thirdly, we need some a priori knowledge of the sea floor morphology: the direction of the sand wave pattern to define the grid, second order stationarity for the morphological residuals of the area to describe the covariance function of the residuals, and uniform morphodynamics per grid line to estimate dynamics correctly. A pattern will only be detected if it is regular enough to be approximated by a sine function. 
The auto-covariance function initially serves to quantify correlations between measurements caused by the measurement and interpolation processes. For simplicity, all covariances are often assumed zero [e.g. Calder, 2003]. This assumption is incorrect if e.g. significant water level reductions have to be applied, as is the case for the Southern North Sea (see Section 3.3.2). Water level reductions have by far the largest impact on the error budget [Dorst, 2004].

However, a simplified modeling of the initial variances and covariances has only limited impact, as the estimation of variance and covariance components changes the initial covariance function into a combined function for uncertainty in all errors and the residual morphological variation. Such an auto-covariance function, or the equivalent semi-variogram, is known in hydrography to characterize morphological variation [e.g. Robert and Richards, 1988], or to estimate the weights of the interpolation procedure known as Kriging [e.g. Calder, 2006; Chilès and Chauvet, 1975].

\subsubsection{Dimensions}

In a two-dimensional space, four different approaches are possible. A zerodimensional approach describes depth at a point $(x, y)$ independent of the surrounding depth values. A one-dimensional approach defines cross-sections in the directions $x$ or $y$, and a two-dimensional approach defines a surface. An analysis in one dimension in the crest direction would not have given us any sand wave parameters, but could be useful to analyze the behaviour of one crest or trough in relation to the others.

Although results of the other approaches are also available, we presented our approach here for one dimension in $x$-direction only. The best approach is discussed in Chapter 6. If we would have chosen a zero-dimensional approach for this combination of dynamics, the detected dynamics would have been highly unorganized, and very difficult to interpret. Such an approach is useful though to identify dynamic sub-areas of the grid, and consequently to redefine the extent of areas correspondingly.

In the case of a two-dimensional approach, we would not have been able to adapt the wavelength per grid line, which would have resulted in larger residuals for less regular areas, like the one used in our example. This causes larger elements in the covariance matrix used as input for step 2, which in turn reduces the power of the tests, and makes the estimates less accurate. The dynamics defined in the example would not have all been found by a two-dimensional analysis.

\subsubsection{Interfering patterns}

For patterns that are two-dimensional, or for two interfering patterns in different directions, the characterization of a single pattern direction is insufficient. Such interfering patterns are found by Knaapen et al. [2001]. Wave crests with limited length potentially migrate in a different direction than perpendicular to the crest direction, which remains undetected with the current spatial representation. If there is a clear difference in scale of the patterns, the size of the analyzed 
grids influence the pattern that is selected as dominant. A pattern with a long wavelength is represented by a slope for short grid lines, and a pattern with a short wavelength is represented by morphological residuals for long grid lines.

For two interfering patterns, one-dimensional analyses in both the pattern directions could be made, instead of a single analysis in the direction of highest variability. Usually, the direction of highest variability does not correspond to any of the two interfering patterns. An option for the two-dimensional analysis is the definition of a spatial representation of two sand waves with different wavelengths and in different directions. Sand waves with a limited crest length could be represented by a second perpendicular wavelength. This way, alongcrest migration is also detected, as reported by e.g. Van Dijk and Egberts [2008] for the Netherlands Continental Shelf.

\subsubsection{Design of the testing procedure}

In Section 3.2.5, we applied the B-method, on which we based our approach. We differ from the original B-method in two ways. In the first place, the B-method uses an overall test in every iteration, to decide if another extension is needed. In the second place, the B-method restarts the iteration procedure for the selection of the characterization, after the extension of the covariance matrix. The first adaptation in the procedure is made because we do not use the extensions to describe single blunders and systematic errors in the measurements, but to describe a more complex physical situation, by detecting as many types of dynamics as possible. The second adaptation is made because we do not assume that the reason for an extension is an additional component in our error budget, but the inevitably incomplete characterization of the sea floor.

Instead of adding more extensions to the characterization until the overall test is accepted, we describe the remaining variability using additional variance and covariance components for these morphological residuals. In this way, we do not introduce unnecessary parameters. The consequences are that it is easier to detect dynamics for a more regular sea floor, and that the detected dynamics are described more accurately for such a sea floor.

\subsubsection{Design of the morphological representation and dispersion}

There are two approaches to the calculation of variances and covariances. The $a$ priori approach is able to calculate covariances before the actual measurements are done. If the covariances of all uncorrelated components are known beforehand, the combined covariance is the sum of the covariances. The calculation of $c^{(\mathrm{e})}(\mathbf{h})$ from the error components of Appendix 3.A is an example of that. The other approach is the a posteriori approach, which calculates covariances from the residuals $\underline{r}_{p, s}^{(\mathrm{e})}$.

The estimation of variances and covariances means a switch from the a priori to the a posteriori approach, by replacing $\mathbf{C}_{d \text {,old }}$ by $\underline{\mathbf{C}}_{d \text {,new }}$. This implies we may not take some systematic error components into account anymore, that are part of the a priori error. Because these systematic components vary little over the 
area considered, they are included in the estimation of the morphological trend, and are therefore not present in the residuals anymore.

We distinguish between planar and sand wave parameters, because of correlations between the parameters within each group. If the grid line does not contain exactly an integer number of sand waves, the sand wave and the planar parameters are correlated as well, causing false detections. This effect is especially prominent because we generally work with areas that have a size of a few times the wavelength only. A decrease of the levels of significance can improve the detection process, meanwhile increasing the risk of missing true dynamics.

The introduction of additional sea floor parameters might improve the analysis: the modeling of sand wave asymmetry, or additional extensions for more types of dynamics. However, an increase in the number of parameters of a characterization, without changes to the amount or uncertainty of the depth data, will decrease the test quotients and thereby the power of each test, and also increase the uncertainty of the estimate of each parameter.

\subsubsection{Relation to morphodynamic models}

A first morphodynamic application of this method is the estimation of pattern migration. A linear trend in time is not only estimated from the positions of crests and troughs, but the shift of the pattern as a whole is considered. Appendix 3.B shows that a linear trend in sand wave migration can only be approximated by a linear trend in $A^{(\mathrm{c})}$ and $A^{(\mathrm{s})}$ for a limited time span, and that the maximum value of outliers is limited.

A second morphodynamic application is the estimation of pattern growth. The estimate for amplitude $A$ does not describe the total amount of spatial variation within the area, as its value depends largely on the regularity of the pattern. For example, compare the depth variations in the example with the estimated reference amplitudes. Consequently, a change in sand wave regularity would appear as a falsely detected change in amplitude.

Idealized, process-based morphological models have been applied to sand wave areas in the Southern North Sea by Van der Veen et al. [2006] and Hulscher and Van den Brink [2001]. Process-based models are able to explain linear migration from the flow and sediment characteristics of the physical system [Németh et al., 2007; Besio et al., 2008]. Unlike our representation for linear growth, model predictions for sand wave growth are initially exponential in those models, usually damped by a higher order term for larger amplitudes. For instance, the non-linear Landau equation is a common description of such growth [Knaapen and Hulscher, 2002]. Parts of this growth curve can be approximated by a linear trend, especially if the sand waves have almost reached their equilibrium heights. We should keep the time span limited, though, and we should not combine surveys from before and after a dredging operation in an analysis.

Introducing a Landau equation for sand wave growth implies that we will have to apply a linearization first. Knaapen et al. [2005] show that such a change improves the approach of Wüst [2004]. We expect that an improvement could 
be made for the estimation of sand wave growth with deformation analysis as well.

Extreme meteorologic conditions and human interventions cause sudden changes in morphology [Terwindt, 1971; Knaapen and Hulscher, 2002]. In the present design, such a change is described by a series of outliers, which gives useful information on such an event. For an optimal estimation of the regeneration of a pattern, it is possible to formulate a specific extension, by a trend that starts after an extreme event. A series of outlying surveys is an indication for this type of behaviour.

\subsection{Conclusion}

We have presented a statistical procedure to detect and estimate sea floor dynamics. A test scenario was set up as a realistic situation for the Southern North Sea: the morphology is based on measured data, and the morphodynamics were inspired by results of others [Wright, 1992; Langhorne, 1982b; Terwindt, 1971]. The presented results clearly show that a simple morphological representation can be used for the analysis of sea floor dynamics of the morphologically complicated area that we presented, as the sea floor dynamics from the test scenario were detected and estimated well.

Therefore, we conclude that the monitoring of areas of the sea floor using deformation analysis is a useful method to acquire insight into the past behaviour of the sea floor, even for morphologically complicated areas. The method gives parameter estimates for the deformations that include their uncertainties. A characterization that consists of a sloping plane and a sinusoidal wave is sufficient to analyze dynamics in tidal sand wave areas, including sand wave growth and migration. Such a characterization gives a good balance between a limited number of parameters and limited parameter uncertainty.

\section{Appendices}

\section{A Error characteristics}

The error $e(x, y, t)$, see equation (3.1), at point $p$, and during survey $s$ is denoted $\underline{e}_{p, s}$. It is the sum of several error sources:

$$
\underline{e}_{p, s}=\underline{e}_{p, s}^{(\mathrm{v})}+\underline{e}_{p, s}^{(\mathrm{h})}+\underline{e}_{p, s}^{(\mathrm{k})}
$$

These sources are:

1. the combined influence of all processes involved in depth measurement $\underline{e}_{p, s}^{(\mathrm{v})}$

2. the influence of horizontal position measurement on depth $\underline{e}_{p, s}^{(\mathrm{h})}$, due to the incorrect location;

3. the potential influence of interpolation $\underline{e}_{p, s}^{(\mathrm{k})}$, using e.g. Kriging. 
To describe the error covariances $c_{s}^{(\mathrm{k})}$, we assume the spatially statistical constraint of stationarity is satisfied. The error is first order stationary, because its expected value $\mathrm{E}\{\underline{\underline{e}}\}$ equals zero for every position $\left(x_{p}, y_{p}\right)$. It is also second order stationary, if, in addition, the covariances between the errors do not depend on position, but only on the distance vector $\mathbf{h}$, which has magnitude $\|\mathbf{h}\|=h$. The auto-covariance between measurement errors of survey $s$ at two positions is then expressed as $c_{s}^{(\mathrm{e})}(\mathbf{h})$, for all $\mathbf{h}$ including the variance $c_{s}^{(\mathrm{e})}(0)=\sigma_{s}^{(\mathrm{e}) 2}$.

The variance is a discontinuity in the covariance function if parts of the error budget are uncorrelated between measurements, which means that the variance is larger than any value of the covariance function. This is called a nugget effect. The auto-covariance function is required to be positive-definite. We assume second-order stationarity for $h \neq 0$ only, and therefore define $h>0$. Hence, we allow for the calculation of a variance $\sigma_{p, s}^{(\mathrm{e}) 2}$ per depth value, as is often necessary in hydrography. For instance, the uncertainty of a depth measurement depends on depth itself [International Hydrographic Organization, 2008a].

\section{B Transformation of the sand wave parameters}

The deformation analysis leads to estimates $\underline{A}^{(\mathrm{c})}$ and $\underline{A}^{(\mathrm{s})}$ of the provisional sand wave parameters $A^{(\mathrm{c})}$ and $A^{(\mathrm{s})}$, along with their variances $\sigma^{(\mathrm{c}) 2}$ and $\sigma^{(\mathrm{s}) 2}$, and their mutual covariance $c\left(\underline{A}^{(\mathrm{c})}, \underline{A}^{(\mathrm{s})}\right)$. The original parameters, i.e. the sand wave amplitude $A$ and the horizontal crest position $\xi$ that are defined in equation (3.8), then follow from the transformation

$$
\mathbf{f}=\left[\begin{array}{c}
A \\
\xi
\end{array}\right]=\left[\begin{array}{c}
\sqrt{A^{(\mathrm{c}) 2}+A^{(\mathrm{s}) 2}} \\
\arctan \left(A^{(\mathrm{s})} / A^{(\mathrm{c})}\right) / \kappa
\end{array}\right] .
$$

Because this is a nonlinear transformation, the detection of linear trends in the provisional parameters $A^{(\mathrm{c})}$ and $A^{(\mathrm{s})}$ does not correspond to the detection of linear trends in the original parameters $A$ and $\xi$. Instead, this leads to a nonlinear evolution of both $A$ and $\xi$ (Figure 3.17, top row). Conversely, a linear trend in the original parameters $A$ and $\xi$ implies nonlinear behaviour of both $A^{(\mathrm{c})}$ and $A^{\text {(s) }}$ (Figure 3.17, bottom row). However, for realistic values of growth and migration, these nonlinearities only become visible on very large time scales. On realistic time scales of approximately a decade, the curves in Figure 3.17 are safely approximated by linear relationships.

Linear trends in the provisional parameters thus lead to linearized trends in the original parameters. The corresponding trend parameters are given by

$$
\dot{\mathbf{f}}=\left[\begin{array}{c}
\dot{A} \\
\dot{\xi}
\end{array}\right] \approx \mathbf{F}\left[\begin{array}{c}
\dot{A}^{(\mathrm{c})} \\
\dot{A}^{(\mathrm{s})}
\end{array}\right]
$$

in which $\mathbf{F}$ is the Jacobian matrix of the function $\mathbf{f}$ in equation (3.28), given by

$$
\mathbf{F}=\left[\begin{array}{cc}
\frac{A^{(\mathrm{c})}}{\sqrt{A^{(\mathrm{c}) 2}+A^{(\mathrm{s}) 2}}} & \frac{A^{(\mathrm{s})}}{\sqrt{A^{(\mathrm{c}) 2}+A^{(\mathrm{s}) 2}}} \\
-\frac{A^{(\mathrm{s})}}{\kappa\left(A^{(\mathrm{c}) 2}+A^{(\mathrm{s}) 2}\right)} & \frac{A^{(\mathrm{c})}}{\kappa\left(A^{\left.(\mathrm{c})^{2}+A^{(\mathrm{s}) 2}\right)}\right.}
\end{array}\right] .
$$



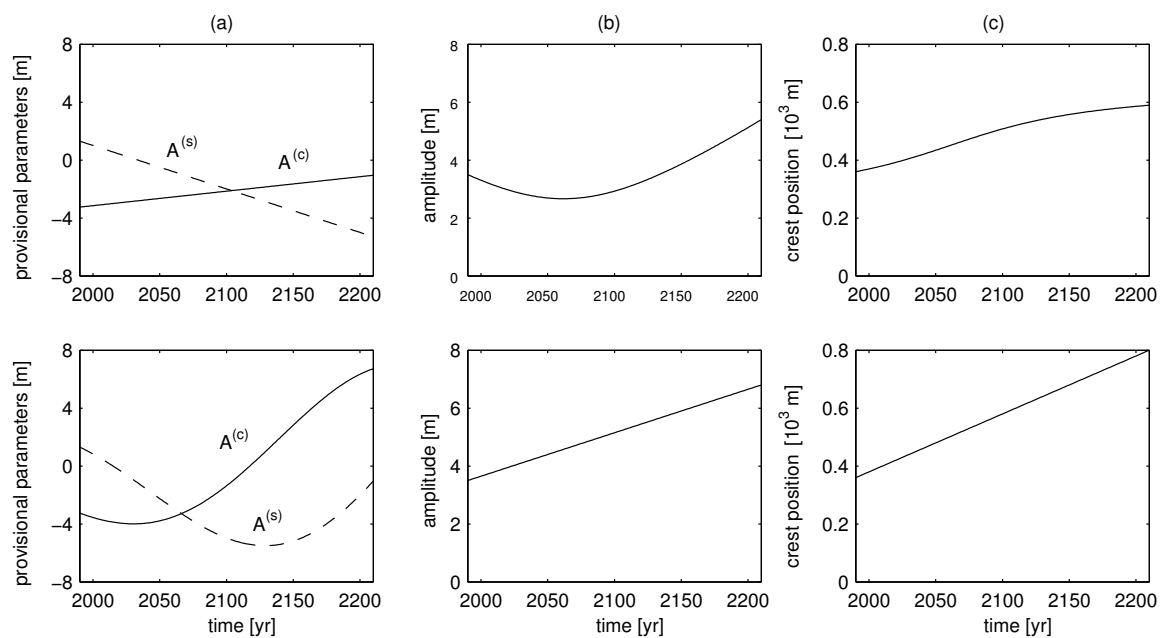

Figure 3.17: The effects of linear trends in the provisional parameters $A^{(\mathrm{c})}$ and $A^{(\mathrm{s})}$ on the evolution of the original parameters $A$ and $\xi$, and vice versa. Top row: (a) imposing linear trends on $A^{(\mathrm{c})}$ of $+0.01 \mathrm{~m} / \mathrm{yr}$ (solid), and $A^{(\mathrm{s})}$ of $-0.03 \mathrm{~m} / \mathrm{yr}$ (dashed) leads to nonlinear evolution of both (b) $A$ and (c) $\xi$. Bottom row: (b) imposing an increase in sand wave amplitude of $+0.015 \mathrm{~m}$ per year, and (c) a migration of $+2 \mathrm{~m}$ per year leads to a nonlinear evolution of (a) $A^{(\mathrm{c})}$ (solid) and $A^{(\mathrm{s})}$ (dashed) in time.

Also with respect to outliers, we may linearize to transform an outlier in terms of the provisional parameters into an outlier in terms of the original parameters:

$$
\Delta \mathbf{f}_{s}=\left[\begin{array}{c}
\Delta A_{s} \\
\Delta \xi_{s}
\end{array}\right] \approx \mathbf{F}\left[\begin{array}{c}
\Delta A_{s}^{(\mathrm{c})} \\
\Delta A_{s}^{(\mathrm{s})}
\end{array}\right]
$$

To warrant the validity of this linearization, the deviation of an outlier should be small with respect to the general values.

Using equation $(3.28)$ to transform the provisional parameter estimates $\underline{A}^{(\mathrm{c})}$ and $\underline{A}^{(\mathrm{s})}$ back to the original parameter estimates $\underline{A}$ and $\underline{\xi}$, their variances propagate according to

$$
\mathbf{C}_{\mathbf{f}}=\mathbf{F}\left[\begin{array}{cc}
\sigma^{(\mathrm{c}) 2} & c\left(\underline{A}^{(\mathrm{c})}, \underline{A}^{(\mathrm{s})}\right) \\
c\left(\underline{A}^{(\mathrm{c})}, \underline{A}^{(\mathrm{s})}\right) & \sigma^{(\mathrm{s}) 2}
\end{array}\right] \mathbf{F}^{\mathrm{T}} .
$$

The matrix $\mathbf{C}_{\mathbf{f}}$ contains the variances of the original estimates, as well as their covariance. The transformations of the sand wave change parameters, presented in equations (3.29) and (3.31), use a similar relation for the propagation of their variances and covariances.

The covariance matrix of the estimates for the final parameters $\underline{A}^{\prime}$ and $\underline{\xi}^{\prime}$ in vector $\underline{\mathbf{f}}^{\prime}$ follows from their relation with the original parameters estimates $\underline{A}$ 
and $\underline{\xi}$ in vector $\underline{\mathbf{f}}$ :

$$
\begin{aligned}
\underline{\mathbf{f}}^{\prime} & =\left[\begin{array}{cc}
1 / \underline{A}_{\mathrm{ref}} & 0 \\
0 & 1
\end{array}\right] \underline{\mathbf{f}}-\left[\begin{array}{c}
0 \\
\underline{\xi}_{\mathrm{ref}}
\end{array}\right] ; \\
\mathbf{C}_{\mathbf{f}} & =\left[\begin{array}{cc}
1 / \underline{A}_{\mathrm{ref}} & 0 \\
0 & 1
\end{array}\right] \mathbf{C}_{\mathbf{f}}\left[\begin{array}{cc}
1 / \underline{A}_{\mathrm{ref}} & 0 \\
0 & 1
\end{array}\right] .
\end{aligned}
$$

Note that the transposed-symbol of the last matrix of the second line has been omitted, because this matrix equals its transpose.

\section{C The application of statistical estimation and testing}

\section{C.1 Introduction}

Statistical estimation and testing has many different applications [Stuart et al., 1999; Lehmann, 1986]. One of them is the analysis of a series of surveys [Teunissen, 2000, 2001].

The estimation of the parameters of a specific representation is treated in Appendix 3.C.2. The significance of a set of parameters is assessed using statistical tests in Appendix 3.C.3. The approach to test several sets of parameters is given in Appendix 3.C.4. Thereafter, overall fit of the representation is tested by an overall test, as shown in Appendix 3.C.5. Minimal detectable biases are introduced in Appendix 3.C.6, with the aim to define levels of significance in Appendix 3.C.7. These levels of significance are valid for one degree of freedom. To allow for the comparison of several extensions with different numbers of degrees of freedom, we follow the procedure of Appendix 3.C.8.

\section{C.2 Parameter estimation}

Our estimations are Best Linear Unbiased Estimations (BLUE), meaning that the estimates $\underline{\hat{\mathbf{u}}}$ have minimal variance, depend linearly on the data, and that the expected value of the estimates equals the mean. If the measured depth values have a Gaussian distribution, a best estimator equals the most likely estimator (MLE). In this case, adjustment theory states that the BLUEs of the sea floor parameters are calculated as the least squares solution

$$
\underline{\hat{\mathbf{u}}}=\mathbf{C}_{u} \mathbf{A}^{\mathrm{T}} \mathbf{C}_{d}^{-1} \underline{\mathbf{d}} ; \mathbf{C}_{u}=\left(\mathbf{A}^{\mathrm{T}} \mathbf{C}_{d}^{-1} \mathbf{A}\right)^{-1} \text {. }
$$

The $N \times N$ covariance matrix $\mathbf{C}_{u}$ is the covariance matrix of the estimators, having their variances $\sigma_{u}^{2}$ on the main diagonal. The calculation of the dynamics of the amplitude and crest position, as well as their variances, are further discussed in Appendix 3.B.

The estimators $\underline{\hat{\mathbf{m}}}$ for the depth values give the morphological characterization. They are calculated by

$$
\underline{\hat{\mathbf{m}}}=\mathbf{A} \underline{\hat{\mathbf{u}}} ; \mathbf{C}_{m}=\mathbf{A C}_{u} \mathbf{A}^{\mathrm{T}} .
$$

The estimated residuals are:

$$
\underline{\hat{\mathbf{r}}}=\underline{\mathbf{d}}-\underline{\hat{\mathbf{m}}} ; \mathbf{C}_{r}=\mathbf{C}_{d}-\mathbf{C}_{m} .
$$


The values of these estimated residuals in relation to the covariance matrix $\mathbf{C}_{d}$ will influence the decision whether the representation is sufficient, and which parameters improve the representation most.

\section{C.3 Extension testing}

In Section 3.2.4, we introduced the original representation $R_{0}$ (or $R^{(0)}$ ) and alternative representations $R_{a}$ (or $R^{(a)}$ ). To find the best characterization of the sea floor, we compare the original representation $R_{0}$ to an alternative representation $R_{a}$ by a statistical test. In this context, we speak of the test of the null hypothesis against an alternative hypothesis. The additional parameters used in the alternative hypothesis are put in a $Q \times 1$ vector $\mathbf{v}$, and their coefficients in a $M \times Q$ matrix $\mathbf{B}$. The original coefficient matrix and parameter vector are denoted $\mathbf{A}_{0}$ and $\mathbf{u}_{0}$. The product $\mathbf{B}_{a} \mathbf{v}_{a}$ forms the extension of $R_{a}$ :

$$
\begin{aligned}
& R_{0}: \mathrm{E}\{\underline{\mathbf{d}}\}=\mathbf{A}_{0} \mathbf{u}_{0} \\
& R_{a}: \mathrm{E}\{\underline{\mathbf{d}}\}=\mathbf{A}_{0} \mathbf{u}_{0}+\mathbf{B}_{a} \mathbf{v}_{a} .
\end{aligned}
$$

The number of new parameters $Q$ is called the number of degrees of freedom of the extension.

Both the hypotheses are so-called composite hypotheses, which require a generalized likelihood ratio (GLR) test. First, we estimate the parameters of $R_{0}$, see Appendix 3.C.2. The test statistic $\underline{T}_{a}$ for representation $R_{a}$ is based on the residuals of the estimation procedure:

$$
\underline{T}_{a}=\underline{\hat{\mathbf{r}}}^{\mathrm{T}} \mathbf{C}_{d}^{-1} \mathbf{B}_{a}\left(\mathbf{B}_{a}^{\mathrm{T}} \mathbf{C}_{d}^{-1} \mathbf{C}_{r} \mathbf{C}_{d}^{-1} \mathbf{B}_{a}\right)^{-1} \mathbf{B}_{a}^{\mathrm{T}} \mathbf{C}_{d}^{-1} \underline{\hat{\mathbf{r}}} .
$$

This test statistic has a $\chi^{2}(Q, 0)$-distribution if $R_{0}$ is true, and a $\chi^{2}\left(Q, \lambda_{a}\right)$ distribution if a $R_{a}$ is true. The noncentrality parameter $\lambda_{a}$ is given by:

$$
\lambda_{a}=\mathbf{v}_{a}^{\mathrm{T}} \mathbf{B}_{a}^{\mathrm{T}} \mathbf{C}_{d}^{-1} \mathbf{C}_{r} \mathbf{C}_{d}^{-1} \mathbf{B}_{a} \mathbf{v}_{a}
$$

More details on $\lambda_{a}$ are given in the appendices 3.C.6, 3.C.7 and 3.C.8.

The test statistic $\underline{T}_{a}$ is compared to a critical value $k: R_{a}$ is accepted if

$$
\underline{T}_{a}>k .
$$

If $R_{0}$ is true, it can still be rejected with probability $\alpha$, due to the misleading influence of the vector of the measurement errors and residuals, $\underline{\mathbf{r}}$. The probability $\alpha$ is called the level of significance. We specify $\alpha$ according to Appendix 3.C.7, and subsequently $k_{\alpha}$ follows from evaluating $\chi^{2}(Q, 0): \alpha$ is the integral of $\chi^{2}(Q, 0)$ over $k \geq k_{\alpha}$. A small level of significance $\alpha$ is desirable, but it implies a large probability $\beta$ that $R_{0}$ is accepted although, in fact, $R_{a}$ is true. Therefore, a suitable value for $k_{\alpha}$ depends on a trade-off between these two risks. A test that maximizes the power $\gamma=1-\beta$ for a given $\alpha$ is called most powerful. The power is the probability of correctly accepting representation $R_{a}$. 


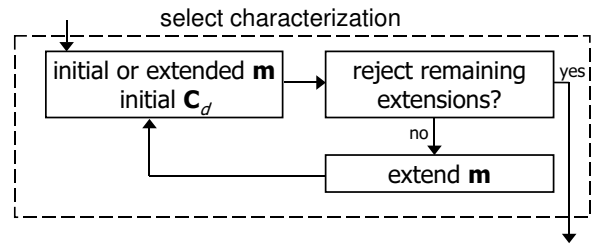

Figure 3.18: Flow diagram for the selection of the characterization. This diagram is a more detailed view of the top panel of Figure 3.4.

\section{C.4 Subsequent acceptance of extensions}

Our approach allows us to compare tests statistics with different degrees of freedom $Q$ simultaneously. Also, different levels of significance $\alpha$ can be chosen for each extension. This way, we determine the characterization by combining extensions, which is the first part of the process (Figure 3.4). This part is shown in more detail in Figure 3.18.

As both these parameters $\alpha$ and $Q$ influence the critical value $k$, we need to consider the test statistics $\underline{T}_{a}$ in relation to $k$. To this end, we define the test quotient $\underline{q}_{a}$ :

$$
\underline{q}_{a}=\underline{T}_{a} / k_{\alpha, Q}
$$

The most likely representation $R_{a}$ is the one with the largest $\underline{q}_{a}$, provided all extensions have the same number of degrees of freedom $Q$. The characterization is accepted if its test quotient is larger than one.

If the extensions have a different $Q$, the procedure of Appendix 3.C.8 is followed. According to this procedure, the most likely hypothesis is the one with the largest $\underline{q}_{a}$, where $\alpha$, and therefore $k_{\alpha}$, depends on $Q$. Representation $R_{0}$ is replaced by representation $R_{a}$ if

$$
\underline{q}_{a}>1
$$

As shown in Figure 3.18, the remaining extensions are evaluated again after replacing the representation $R_{0}$ by an $R_{a}$. For example, after the $i$-th iteration, the previous representation $R_{a_{1}, a_{2}, \cdots, a_{i-1}}$ is updated to the next representation $R_{a_{1}, a_{2}, \cdots, a_{i}}$, using extension $\mathbf{B}_{a_{i}} \mathbf{v}_{a_{i}}$. This procedure continues until $\underline{q}_{a} \leq 1$ for all remaining extensions. The iteration process also stops if all possible extensions have subsequently been accepted. As an example, the selection process of the representation for step 1 is given in Figure 3.5.

\section{C.5 The overall test and the final estimation}

The overall test follows from the selection of the characterization, as it decides whether it is necessary to add new components to the dispersion (Figure 3.4). This is shown in Figure 3.19. The overall test is the widest test possible. In this case, we have $Q=M-N$ degrees of freedom. This implies that we test the 


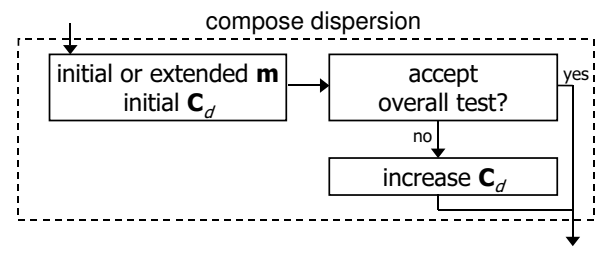

Figure 3.19: Flow diagram for the composition of the dispersion. This diagram is a more detailed view of the middle panel of Figure 3.4.

final representation, having an $N \times 1$ parameter vector, against an alternative representation that has a $M \times 1$ parameter vector of the same length as the vector of depth values.

The calculation of the overall test statistic $T^{(\mathrm{o})}$ follows equation (3.38). It simplifies to

$$
\underline{T}^{(\mathrm{o})}=\underline{\hat{\mathbf{r}}}^{\mathrm{T}} \mathbf{C}_{d}^{-1} \underline{\hat{\mathbf{r}}} .
$$

We define its quotient

$$
\underline{q}^{(\mathrm{o})}=\underline{T}^{(\mathrm{o})} /(M-N)
$$

as a test statistic for the overall test. This test statistic has a central Fisher distribution $F(M-N, \infty)$. In Section 3.D.5, we will use this test statistic $\underline{q}^{(\mathrm{o})}$ for the variance component estimation.

The representation is accepted if

$$
\underline{q}^{(\mathrm{o})} \leq 1
$$

which means that the residuals $\underline{\hat{\mathbf{r}}}$ are that small that they are sufficiently described by covariance matrix $\mathbf{C}_{d \text {,old }}$. The dispersion remains equal to this matrix:

$$
R_{a, \text { old }}: \mathrm{D}\{\underline{\mathbf{d}}\}=\mathbf{C}_{d, \text { old }} .
$$

If the representation is rejected, we add new components to the elements of this covariance matrix, in such a way that the overall test is accepted. This results in matrix $\mathbf{C}_{d, \text { new }}$ :

$$
R_{a, \text { new }}: \mathrm{D}\{\underline{\mathbf{d}}\}=\mathbf{C}_{d, \text { new }} .
$$

The two representations $R_{a, \text { old }}$ and $R_{a, \text { new }}$ are the ones without and with an added covariance component, respectively. The estimation of additional covariance components is described in Appendix 3.D.

The resulting representation is used for another adjustment as in equation (3.34). This gives us the final estimates for the parameters in $\underline{\hat{\mathbf{u}}}$ and their uncertainties $\underline{\mathbf{C}}_{u}$ (Figure 3.20), which finalizes the estimation procedure. 
calculate final estimates and their uncertainty

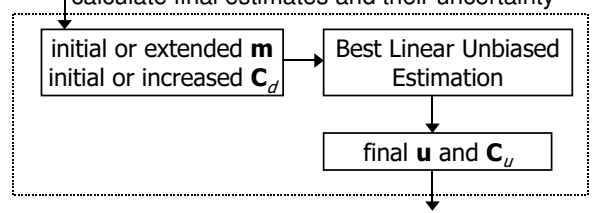

Figure 3.20: Flow diagram for the calculation of the final estimates and their uncertainties. This diagram is a more detailed view of the bottom panel of Figure 3.4.

\section{C.6 Definition of minimal detectable biases}

In case of an extension with one degree of freedom, $Q=1$, the matrix $\mathbf{B}_{a}$ reduces to an $M \times 1$ vector $\mathbf{b}_{a}$, and the vector $\mathbf{v}_{a}$ to a single parameter $v_{a}$, see equation (3.37). Using equation (3.39), we find a relation between the value of $v_{a}$ that is minimal detectable for a criterion with a probability of the power $\gamma$ :

$$
\left|v_{a}\right|=\sqrt{\frac{\lambda_{a}}{\mathbf{b}_{a}^{\mathrm{T}} \mathbf{C}_{d}^{-1} \mathbf{C}_{r} \mathbf{C}_{d}^{-1} \mathbf{b}_{a}}}
$$

The noncentrality parameter $\lambda_{a}$ depends on the chosen level of significance $\alpha$ of the test, the definition of the extension (via its number of degrees of freedom $Q)$, and the power $\gamma$ of the test: $\lambda=\lambda(\alpha, Q, \gamma)$. Setting the power $\gamma$ to $50 \%$, like in Appendix 3.C.8, and with $Q=1$, we find a relation between the Minimal Detectable Bias (MDB) and the level of significance: $\left|v_{a}\right|=\left|v_{a}\right|(\alpha)$.

This relation can be used to make statements like: "if we test using a level of significance $\alpha$, a bias of magnitude $\left|v_{a}\right|$ can be found with a probability of power $\gamma$. . Larger biases can be detected with a higher probability, and smaller biases with a smaller probability. Even a very small bias can be found, with a small probability, but a very large bias will remain undetected with a certain small probability as well.

\section{C.7 Levels of significance for specific criteria with one degree of freedom}

The choice of the levels of significance $\alpha_{a}$ for the various alternative representations $R_{a}$ determines the critical values $k_{\alpha}$, and thereby directly influences the values of the test quotients $\underline{q}_{a}$. For every level of significance, an MDB can be calculated. This quantity helps us to find suitable levels of significance for specific morphological criteria.

Consider, as an example, a sea floor without a slope, but with a sand wave pattern that is perfectly sinusoidal along a grid line of $1000 \mathrm{~m}$ length. The sand wave has a wavelength $L$ of $750 \mathrm{~m}$ and does not migrate. This sand wave is described by the single parameter $A$, its amplitude, and the sand wave extensions have one degree of freedom only, denoted by $Q=1$. 
Depth values are available at a grid with a spacing of $50 \mathrm{~m}$. Their $95 \%$ confidence limit is set at according to the IHO S44 order 1 standard (see Section 3.3.2) for a bed level of $40 \mathrm{~m}$ at $0.72 \mathrm{~m}$, so the variance of the measurements is $\sigma^{(\mathrm{e}) 2}=0.13 \mathrm{~m}^{2}$. The covariance function $c^{(\mathrm{e})}(\mathbf{h})$ of the measurements is

a Gaussian function, with its inflection point $h_{x}^{(\mathrm{i}, \mathrm{e})}$ at $2500 \mathrm{~m}$ and the covariance at very small distances $c_{0}^{(\mathrm{e})}$ at $0.01 \mathrm{~m}^{2}$, corresponding to a $95 \%$ confidence limit of $0.2 \mathrm{~m}$. A grid line is measured six times with an interval of two years, so the total time interval is 10 years.

We have the following criteria for the spatial sand wave test, the linear sand wave trend test, and the sand wave outlier test:

1. criterion for sand wave test: the minimal detectable amplitude $|A|$ should be $0.25 \mathrm{~m}$ for a single survey, with a probability of $\gamma=50 \%$;

2. criterion for linear trend test: the minimal detectable linear trend in time for the amplitude $|\dot{A}|$ should be $0.025 \mathrm{~m} / \mathrm{yr}$ or $0.25 \mathrm{~m}$ over the total time interval, with a probability of $\gamma=50 \%$, in the absence of any other dynamics;

3. criterion for outlier test: the minimal detectable outlier for the amplitude $\left|A_{s}\right|$ in survey $s$ should be $0.25 \mathrm{~m}$, with a probability of $\gamma=50 \%$, in the absence of any other dynamics.

The resulting levels of significance $\alpha_{a}$ are $3 \%, 4 \%$ and $3 \%$ for the three criteria, respectively. These results are obtained using the relation $\left|v_{a}\right|=\left|v_{a}\right|(\alpha)$, found in Section 3.C.6. The noncentrality parameter $\lambda_{a}$ is found using equation 3.39, and $\alpha_{a}$ follows from $\lambda_{a}$ using the relation $\lambda=\lambda(\alpha, Q=1, \gamma=50 \%)$, also found in Section 3.C.6. The levels of significance for the spatial slope test, the linear planar trend test, and the planar outlier test are chosen equal to the corresponding sand wave test.

\section{C.8 Levels of significance for extensions with several degrees of freedom}

The B-method for simultaneous testing of several hypotheses with different number of degrees of freedom, and therefore different distribution functions, was developed by Baarda [1968].

The choice of the level of significance $\alpha$ for an hypothesis with $Q$ degrees of freedom follows in several steps from the definition of a level of significance $\alpha_{1}$ for a hypothesis with one degree of freedom. An example for $\alpha_{1}=4 \%$ is given in Figure 3.21. In this process, the noncentrality parameter $\lambda$ plays a central role. As discussed in Appendix 3.C.6, $\lambda=\lambda(\alpha, Q, \gamma)$.

We start with the defined level of significance $\alpha_{1}$ and the known number of degrees of freedom $Q$, and set the power $\gamma$ to $50 \%$, as advised by De Heus et al. [1994]. The steps in this process are:

1. calculate $k_{\alpha, 1}$ : we define an $\alpha_{1}$ for the imaginary situation that the present extension has one degree of freedom, which sets $k_{\alpha, 1}$, as $\alpha_{1}$ is the integral 
of $\chi^{2}(1,0)$ over $k_{\alpha, 1}$ to infinity. The function $\chi^{2}(1,0)$ is shown by the solid line in Figure 3.21a, and $k_{\alpha, 1}$ by the vertical line;

2. calculate $\lambda$ : now, $\lambda(\alpha, 1, \gamma)$ follows from the choice of the power $\gamma$, as $\gamma$ is the integral of $\chi^{2}(1, \lambda)$ over $k_{\alpha, 1}$ to infinity. The function $\chi^{2}(1, \lambda)$ is shown by the dashed line in Figure 3.21a. To obtain distribution functions $\chi^{2}(Q, \lambda)$, for any number of the degrees of freedom $Q$, we keep the noncentrality parameter $\lambda(\alpha, Q, \gamma)$ in $\chi^{2}(Q, \lambda)$ constant, at its value for $\lambda(\alpha, 1, \gamma)$

3. calculate $k_{\alpha, Q}$ : The power $\gamma$ is the integral of $\chi^{2}(Q, \lambda)$ over $k_{\alpha, Q}$ to infinity. This defines the critical value $k_{\alpha, Q}$. Functions $\chi^{2}(Q, \lambda)$ are shown by the dashed lines in Figure 3.21a-c, and $k_{\alpha, Q}$ by the vertical lines;

4. calculate $\alpha_{Q}$ : The level of significance $\alpha_{Q}$ is the integral of $\chi^{2}(Q, 0)$ over $k_{\alpha, Q}$ to infinity. Functions $\chi^{2}(Q, 0)$ are shown by the solid lines in Figure $3.21 \mathrm{a}-\mathrm{c}$.

In Appendix 3.C.7, we used levels of significance of $3 \%$ and $4 \%$. The example of Figure 3.21 uses $\alpha_{1}=4 \%$. The noncentrality parameter $\lambda$ is 4.22 , and the critical values $k_{4 \%, Q}$ are $4.22,5.26$ and 6.29 , for one, two and three degrees of freedom $Q$ respectively. If $\alpha_{1}=3 \%$, the noncentrality parameter $\lambda$ is 4.71 , and the critical values $k_{3 \%, Q}$ are $4.71,5.75$ and 6.77 .

\section{D The estimation of covariances}

In general, variances at a position $(x, y)$, and covariances between positions are stored in a covariance matrix. An iterative procedure for the estimation of components of such a matrix is given in Section 3.D.1. In some cases, it is possible to determine the elements of a covariance matrix in a direct, noniterative, way. For instance, if the covariances describe second-order stationary residuals (Appendix 3.A), we could also express covariances in a covariance function (Section 3.D.2).

In step 1 (Sections 3.D.3 and 3.D.4), we use both the iterative procedure and a non-iterative solution based on the estimation of a covariance function (Section 3.D.1). In step 2 (Section 3.D.5), we use a very simple non-iterative solution based on the overall test (Section 3.C.5).

\section{D.1 The estimation of a covariance matrix}

To include an additional stochastic influence in a covariance matrix $\mathbf{C}_{d \text {,old }}$, the procedure of least squares variance component estimation [Teunissen and AmiriSimkooei, 2008; Amiri-Simkooei, 2007] is available. In this approach, the original covariance matrix $\mathbf{C}_{d \text {,old }}$ is replaced by a new covariance matrix $\mathbf{C}_{d \text {,new }}$. To this end, $K$ cofactor matrices $\mathbf{D}_{d, k}$ are added, which are symmetric and mutually uncorrelated. This is expressed as:

$$
\mathbf{C}_{d, \text { new }}=\mathbf{C}_{d, \text { old }}+\sum_{k=1}^{K} f_{k} \mathbf{D}_{d, k}
$$




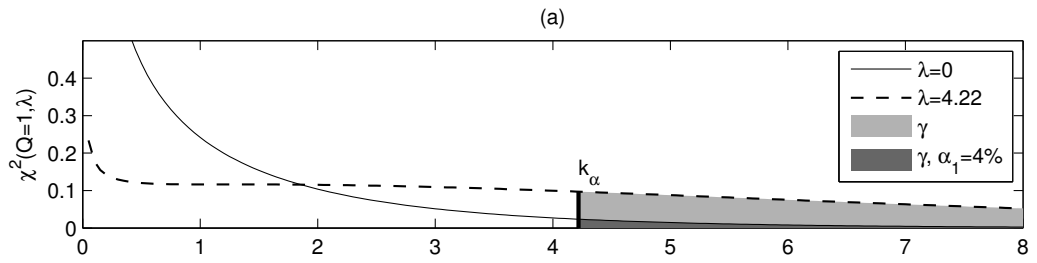

(b)

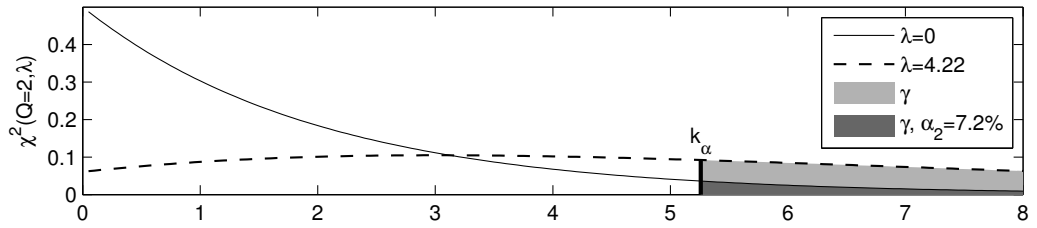

(c)

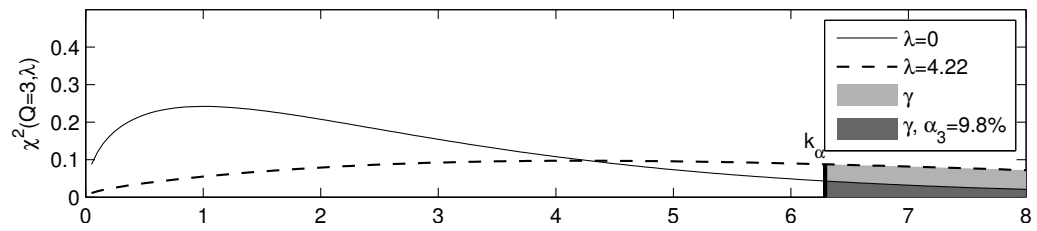

Figure 3.21: Functions describing $\chi^{2}$-distributions, used to illustrate the calculation of critical values for a level of significance of $\alpha_{1}$ of $4 \%$, using a power $\gamma$ of $50 \%$. (a) one degree of freedom; (b) two degrees of freedom; (c) three degrees of freedom.

The relative contribution of each cofactor matrix $\mathbf{D}_{d, k}$ is determined by the scalar factors $f_{k}$. Estimates $\hat{f}_{k}$ for these factors are obtained from the residuals, using a linear least squares procedure, similar to the one given in Appendix 3.C.2, see Teunissen and Amiri-Simkooei [2008]; Amiri-Simkooei [2007]. In this procedure, the covariance matrix $\mathbf{C}_{d \text {,new }}$ has to be used. Therefore, we must calculate the matrix $\mathbf{C}_{d, \text { new }}$ iteratively.

\section{D.2 The estimation of a covariance function}

Let us define $h_{x} \geq 0$ as the distance in $x$-direction. The empirical function of covariances $\underline{c}_{s}^{(\mathrm{emr})}\left[h_{x}\right]$ is derived at discrete distances from the realized residuals $r_{p, s}^{(\mathrm{em})}$. (The letters e, $\mathrm{m}$ and $\mathrm{r}$ between the parentheses denote the influence of measurement error, morphology and the discrete realization respectively, and the square brackets indicate the discrete character of this covariance function.) The covariance function $c_{s}^{(\mathrm{em})}\left(h_{x}\right)$ in this direction follows from the fit of a positive-definite function on the empirical function $\underline{c}_{s}^{(\mathrm{emr})}\left[h_{x}\right]$. More details are found in Chapter 2.

To obtain a sufficient number of pairs of residuals at the required distances, it is necessary to calculate one empirical function for the full grid of $P$ nodes, 
instead of one empirical function per grid line. Hereby, only covariances between grid nodes on the same grid line are taken into account. The maximum distance for which a covariance is calculated is half the maximum grid line length, to guarantee a sufficient number of pairs at all distances. The sampling density of the empirical function equals the spacing of the grid.

The positive definite covariance function $c_{s}^{(\mathrm{em})}\left(h_{x}\right)$ that we use is the product of a Gaussian and a cosine function:

$$
c_{s}^{(\mathrm{em})}\left(h_{x}\right)=c_{0, s}^{(\mathrm{em})} \exp \left(-\left(h_{x} / h_{x, s}^{(\mathrm{i}, \mathrm{em})}\right)^{2}\right) \cos \left(\kappa^{(\mathrm{em})} h_{x}\right) .
$$

The $2 S+1$ parameters involved in this fit are the covariance $\underline{c}_{0, s}^{(\mathrm{em})}$ per survey for distances approximating zero, the Gaussian inflection point $\underline{h}_{x, s}^{(\mathrm{i}, \mathrm{em})}$ per survey, and one wave number $\underline{\kappa}^{(\mathrm{em})}$ for all surveys. The cosine function is necessary to account for the rhythmic patterns in the residuals. This is due to e.g. the approximation of asymmetric sand waves by a sinusoidal function, which results in a wavelength $\underline{L}^{(\mathrm{em})}$ in the residuals. This wavelength $\underline{L}^{(\mathrm{em})}=2 \pi / \underline{\kappa}^{(\mathrm{em})}$ equals then about half the estimated constant wavelength $\underline{L}^{(\mathrm{d})}$ of Section 3.2.5. We first estimate the three parameters $\underline{c}_{0}^{(\mathrm{em})}, \underline{h}_{x}^{(\mathrm{i}, \mathrm{em})}, \underline{\kappa}^{(\mathrm{em})} \underline{\mathrm{for}}$ all surveys, using all data. Hereafter, we use wave number $\underline{\kappa}^{(\mathrm{em})}$ for every survey, and estimate covariances $\underline{c}_{0, s}^{(\mathrm{em})}$ and inflection points $\underline{h}_{x, s}^{(\mathrm{i}, \mathrm{em})}$ again per individual survey.

\section{D.3 The estimation of a covariance matrix in step 1 for a single survey}

The variance component estimation of step 1 uses two cofactor matrices $\mathbf{D}_{d, k}$ per survey, defining the structure of the additional components (Section 3.D.1). This means we use $K=2 S$ cofactor matrices in total, with size $M \times M, M=P S$. Let us first consider the variance component estimation per survey, after which we will present the estimation for all $S$ surveys. The $P \times P$ matrices that only contain the elements at positions $p$ for a single survey $s$ are denoted $\mathbf{C}_{d, s}$ and $\mathbf{D}_{d, s}$.

We assume that the morphological residuals $\underline{r}_{p, s}^{(\mathrm{m})}$ are second order stationary (Appendix 3.A), for distances $h_{x} \geq 0$ with $h_{x}$ the distance in $x$-direction. For errors $\underline{e}_{p, s}$, we made this assumption for $h>0$ only. The assumptions enable us to express the combined covariances per survey as $c_{s}^{(\mathrm{em})}\left(h_{x}\right)$, for $h_{x}>0$, using equation (3.50). The results of the evaluation of this function are given on the non-diagonal elements of a matrix $\mathbf{C}_{d, s}^{(\mathrm{em}, \mathrm{c})}$, for which the diagonal only contains zeros. (The letter c denotes that this matrix only contains covariances for $h_{x}>0$.)

As the errors $\underline{e}_{p, s}$ are not assumed second order stationary for $h_{x}=0$, the variances on the diagonal of the covariance matrix $\mathbf{C}_{d, s}^{(\mathrm{e})}$ are in general not constant. These diagonal elements are used for the matrix $\mathbf{C}_{d \text {,old }}$, that we denote here as $\mathbf{C}_{d, s}^{(\mathrm{e}, \mathrm{v})}$. The morphological variances are given in a separate matrix $\mathbf{C}_{d, s}^{(\mathrm{m}, \mathrm{v})}$. (The letter $\mathrm{v}$ denotes that this matrix only contains variances.) 
The covariance matrix $\mathbf{C}_{d, s}^{(\mathrm{em})}$ therefore consists of the following components:

$$
\mathbf{C}_{d, s}^{(\mathrm{em})}=\mathbf{C}_{d, s}^{(\mathrm{e}, \mathrm{v})}+\mathbf{C}_{d, s}^{(\mathrm{m}, \mathrm{v})}+\mathbf{C}_{d, s}^{(\mathrm{em}, \mathrm{c})}
$$

Firstly, we estimate $\mathbf{C}_{d, s}^{(\mathrm{m}, \mathrm{v})}$, using the iterative procedure of Section 3.D.1. A single factor $\hat{\sigma}_{s}^{(\mathrm{m}) 2}$ is estimated, using the cofactor matrix $\mathbf{D}_{d, s}^{(\mathrm{m}, \mathrm{v})}$ that we define as the $P \times P$ identity matrix:

$$
\underline{\mathbf{C}}_{d, s}^{(\mathrm{em}, \mathrm{v})}=\underline{\mathbf{C}}_{d, s}^{(\mathrm{e}, \mathrm{v})}+\underline{\hat{\sigma}}_{s}^{(\mathrm{m}) 2} \mathbf{D}_{d, s}^{(\mathrm{m}, \mathrm{v})}
$$

An advantage of removing the covariances from $\mathbf{C}_{d s s}^{(\mathrm{e})}$ is that the procedure is less time-consuming without covariances. The resulting factor is the spatially constant morphological variance $\underline{\hat{\sigma}}_{s}^{(\mathrm{m}) 2}$. The combined variances $\underline{\sigma}_{p, s}^{(\mathrm{em}) 2}=\underline{\sigma}_{p}^{(\mathrm{e}) 2}+$ $\underline{\hat{\sigma}}_{s}^{(\mathrm{m}) 2}$ are in general not constant over survey $s$, because the error variances $\underline{\sigma}_{p}^{(\mathrm{e}) 2}$ usually differ per position $p$.

Secondly, we use the positive-definite covariance function of Section 3.D.2 to calculate the elements of $\underline{\mathbf{C}}_{d, s}^{(\mathrm{em}, \mathrm{c})}$, as discussed above. Expressed in the terminology of variance component estimation, the non-diagonal elements of cofactor matrix $\underline{\mathbf{D}}_{d, s}^{(\mathrm{em}, \mathrm{c})}$ are specified using the positive-definite covariance function, with $c_{0, s}=1$, and the diagonal elements are zero. The parameter $\underline{c}_{0, s}$ is the factor. The result is:

$$
\underline{\mathbf{C}}_{d, s}^{(\mathrm{em}, \mathrm{c})}=\underline{c}_{0, s} \underline{\mathbf{D}}_{d, s}^{(\mathrm{em}, \mathrm{c})}
$$

A second advantage of removing the covariances from $\mathbf{C}_{d, s}^{(\mathrm{e})}$ is that it has prevented us from following the iterative procedure for the estimation of the covariances, and instead we use the more straightforward estimation procedure for covariance functions.

\section{D.4 The estimation of a covariance matrix in step 1 for all surveys}

The final covariance matrix $\underline{\mathbf{C}}_{d}^{(\mathrm{em})}$ for all $S$ surveys has size $M \times M$, with $M=P S$. Therefore, the elements of each $P \times P$ matrix $\underline{\mathbf{C}}_{d, s}$ are used as the non-zero elements of an $M \times M$ matrix $\underline{\mathbf{C}}_{d, k}$. The final expression is:

$$
\underline{\mathbf{C}}_{d}^{(\mathrm{em})}=\mathbf{C}_{d}^{(\mathrm{e}, \mathrm{v})}+\sum_{k=1}^{S} \underline{\mathbf{C}}_{d, k}^{(\mathrm{m}, \mathrm{v})}+\sum_{k=1}^{S} \underline{\mathbf{C}}_{d, k}^{(\mathrm{em}, \mathrm{c})}
$$

The overall test statistic $\underline{q}^{(\text {o) }}$ of step 1 decides if an additional morphological component is necessary, therefore we denote it as $\underline{q}^{(\mathrm{o}, \mathrm{m})}$. Because of the adaptation of every element in the old covariance matrix $\underline{\mathbf{C}}_{d}^{(\mathrm{e})}$, the overall test statistic $\underline{q}^{(\mathrm{o}, \mathrm{m})}$ of the new representation $R^{(a, \mathrm{em})}$ equals one, if we would calculate it again after the variance component estimation. The new covariance matrix $\underline{\mathbf{C}}_{d}^{(\mathrm{em})}$ is used as the dispersion of the depth values in step 2 . 


\section{D.5 The estimation of a covariance matrix in step 2}

We use the present covariance matrix $\mathbf{C}_{d}^{(\mathrm{em})}$ both as $\mathbf{C}_{d \text {,old }}$ and as the only cofactor matrix $\mathbf{D}_{d, k}$, and therefore $K$ equals one (Section 3.D.1). The single factor $1+\underline{f}$ equals the overall test statistic $\underline{q}^{(\mathrm{o})}$ of phase 2 . To distinguish it from the overall test quotient of step 1 , we denote it as $\underline{q}^{(\mathrm{o}, \mathrm{t})}$. For step 2 , this means:

$$
\underline{\mathbf{C}}_{d}^{(\mathrm{emt})}=\underline{q}^{(\mathrm{o}, \mathrm{t})} \underline{\mathbf{C}}_{d}^{(\mathrm{em})} .
$$

The single variance factor is always applied, also if it is smaller than one, and therefore the overall test after the variance component estimation is one again, as in step 1 . In case the variance factor is smaller than one, apparently the differences between the residuals of each survey are smaller than described by the covariance matrix. This means the dynamics at small scales are correlated between the surveys. 


\title{
Chapter 4
}

\section{The analysis of migrating tidal sand waves in an anchorage area in the Southern North Sea ${ }^{\star}$}

\begin{abstract}
The analysis of a series of offshore bathymetric surveys provides insight into the morphodynamics of the sea floor, which helps to improve resurvey policies for nautical charting. We show results for the anchorage area Maas West near the Port of Rotterdam, the Netherlands. We use a method called deformation analysis, which is based on statistical testing theory. Our implementation of the method particularly aims to detect dynamics in tidal sand wave patterns. It results in parameter estimates for the sand wave dynamics, and their uncertainty. The area is divided into 18 subareas. The results show that a sand wave pattern is detected for most of the subareas, and a shoreward migration is detected for a majority of them. The estimated migration rates of the sand waves are up to $7.5 \mathrm{~m} / \mathrm{yr}$, with a $95 \%$ confidence interval that depends on the regularity of the pattern. The results agree with previously observed migration rates for the Southern North Sea, and with an idealized process-based model.
\end{abstract}

\subsection{Introduction}

The sea floor of shallow seas is dynamic. For example, rhythmic morphological patterns on sandy beds can change height and migrate [Wright, 1992; Terwindt, 1971], thereby possibly endangering navigation through busy shipping lanes. Such patterns exist on many scales [Knaapen et al., 2001]. In this paper, tidal sand waves are considered, which are widely present throughout the North Sea (Western Europe). They are characterized by a wavelength of hundreds of meters, a height of several meters, and a migration rate of up to several meters per year. The evolution of the sea floor can be interpreted from a series of echo sounder surveys, as has recently been done for the Southern North Sea by e.g. Dorst et al. [2007]; Knaapen [2005]; Van Dijk and Kleinhans [2005]. A method called spatial cross-correlation [Van Dijk and Egberts, 2008; McElroy et al., 2008; Buijsman, 2007; Duffy and Hughes-Clarke, 2005] has recently been used

* This chapter is similar to L. L. Dorst, P. C. Roos, F. M. Sterlini and S. J. M. H. Hulscher. The analysis of migrating tidal sand waves in an anchorage area on the Netherlands Continental Shelf, submitted. 
frequently to calculate migration rates, without using stochastic information on the quality of the depth observations. Methods for the analysis of sea floor dynamics are still in development, as a result of the recent improvements in description of the stochastic character of surveyed depth values of the sea floor. This stochastic character complicates the analysis of its evolution: even modern survey techniques reveal depth with an uncertainty of a few decimeters at best [Wells and Monahan, 2002], due to random errors in the measurement processes.

Frequent bathymetric surveys of shallow seas are necessary to produce reliable nautical charts. The analysis of past surveys aids the planning of resurvey frequencies. Examples of survey plans are given by De Oliveira et al. [2007]; Dehling [2006]; Whatrup et al. [2005]; Dorst [2004]. Resurvey planning is best served by a method that separates true sea floor deformation from measurement uncertainties, and establishes whether a new survey confirms trends found in previous surveys. The description of the uncertainty of surveyed depth values is a subject of growing interest in the hydrographic community [e.g. Calder, 2006; Dorst, 2004], since dealing with uncertainties is crucial to control the quality of the survey process and to interpret bathymetric data. Therefore, we adopt a method that (1) recognizes the stochastic character of surveys, (2) separates static from dynamic behaviour, and (3) can distinguish between different kinds of dynamics. Deformation analysis is such a method.

Deformation analysis applies statistical adjustment theory and testing theory to a series of surveys of deformable objects. We apply the approach of De Heus et al. [1994] to estimate sea dynamics. Details about the method are given in Chapter 3. A comparison with the method of Wüst [2004] for bathymetric trend analysis is given by Lindenbergh et al. [2007]. An overview of general literature on deformation analysis is given by Chrzanowski [2006].

The anchorage area Maas West is located in the Southern North Sea, West of the Port of Rotterdam, as shown in Figure 4.1. It has a relatively high resurvey frequency of once per two years. This frequency is based on its location next to an intensively used shipping lane, in combination with its rather shallow depth of 15 to $25 \mathrm{~m}$. To investigate whether the resurvey frequency could be reduced, the available surveys, measured between 1994 and 2006, were analyzed using deformation analysis. The results indicate sand wave migration. They are compared to earlier results of sand wave migration in the Southern North Sea, given by Knaapen [2005] and Van Dijk and Kleinhans [2005], and to the process-based model of Sterlini et al. [2009].

\subsection{The application of deformation analysis}

\subsubsection{Overview}

In this Section, a brief introduction into the application of the method for two spatial dimensions (analysis of a grid) is given. Similar applications are possible for one (analysis of grid lines) and zero spatial dimensions (analysis of grid nodes). For a description of the method in full detail, see Chapter 3, for analysis in one spatial dimension. 


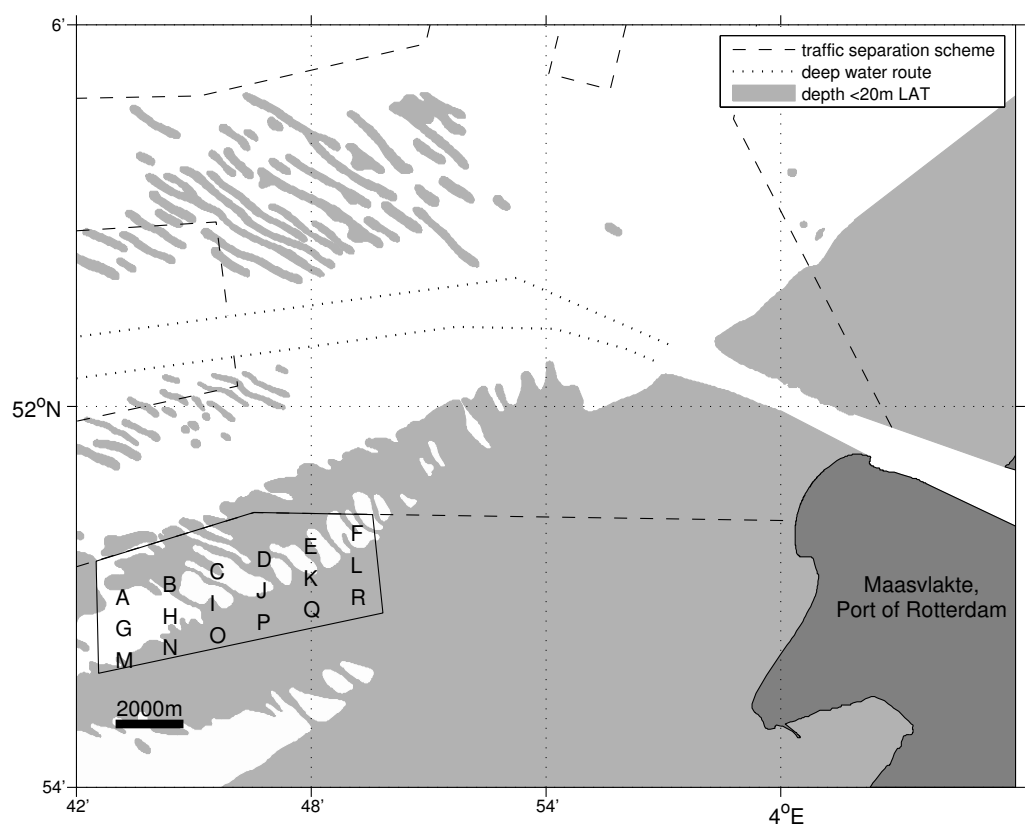

Figure 4.1: Anchorage area Maas West, located in the Southern North Sea in the region West of Rotterdam. It consists of eighteen subareas A to R. The Maasvlakte is the most seaward area of the Port of Rotterdam.

\section{Input and Output}

Deformation analysis is an application of statistical testing theory [e.g. Koch, 1999; De Heus et al., 1994] to analyze series of surveys. Application to the sea floor requires depth values at the nodes of a grid, the uncertainty of each depth value and the time of each survey. The size of the grid is limited to about $20 \times 20$ nodes, for computational reasons. The measurement and interpolation uncertainty is described by an error model, giving variances $\sigma^{(\mathrm{e}) 2}$ and covariance functions $c^{(\mathrm{e})}(\mathbf{h})$ for the used surveys, only dependent on position difference $\mathbf{h}$. Distance $h$ is the length of $\mathbf{h}$.

The procedure allows us to select the significant morphological and morphodynamic parameters for an area, and subsequently provide estimates for these parameters, including confidence intervals. It results in a representation of the measured sea floor that consists of the characterization by a limited number of estimated parameters in a vector $\underline{\hat{\mathbf{u}}}$, and the dispersion of the residual variations from the characterization, which is described by the variances and covariances of the parameters in a matrix $\mathbf{C}_{u}$. The relations between the depth values and the estimated parameters are linear or linearized. This allows us to follow a Best Linear Unbiased Estimation (BLUE) for the estimation of the parameters of the 


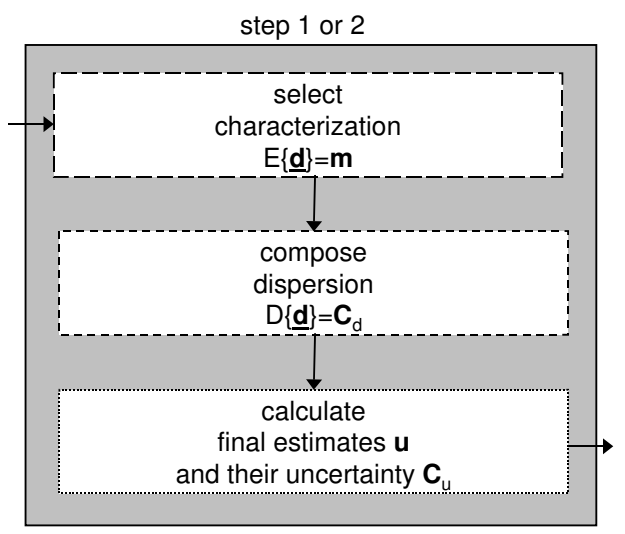

Figure 4.2: Flow diagram of our approach to deformation analysis, modeled after [De Heus et al., 1994]. We first select the characterization and next compose the dispersion. This diagram is a followed in the steps 1 and 2 .

characterization, and for the estimation of their dispersion. The procedure is illustrated in Figure 4.2.

\section{Morphological characterization}

Let us assume the input is available in a coordinate frame $(x, y, z)$, in which the three directions are perpendicular to each other, and depth $d$ during survey $s$ is given as $z=-d_{p, s}$ at locations $\left(x_{p}, y_{p}\right)$. The vector of all measured depth values is denoted $\underline{\mathbf{d}}$, and their variances and mutual covariances are given in $\mathbf{C}_{d}$. Deformation analysis tests extensions to a set of morphological parameters, starting with the simplest characterization possible, which is the single parameter bed level $d^{(\mathrm{b})}$, assumed constant in space and time. Such an extension is accepted if the so-called test quotient $q$ of an extension is the largest over all available extensions, and if this test quotient is larger than one. The characterization is extended until the remaining test quotients are all smaller than one.

In step 1 of the procedure, spatial extensions are considered, and in step 2 temporal extensions. Step 0 prepares the input grids, and step 3 deals with the adaptation of the survey plan based on the results. Also see Figure 4.3 for the steps. This paper gives a methodical introduction to the two shaded steps, and presents and discusses the results of these two steps. Step 0 is treated in Chapter 2, and step 3 in Chapter 6.

The spatial extensions (step 1) to the initial parameter bed level $d^{(\mathrm{b})}$ are (i) two bed slope parameters $\psi^{(\mathrm{x})}$ and $\psi^{(\mathrm{y})}$ in both the horizontal directions, and (ii) two sand wave parameters: amplitude $A$ and crest position $\xi$. Sand wave patterns are assumed to be regular, such that a horizontal direction of the pat- 


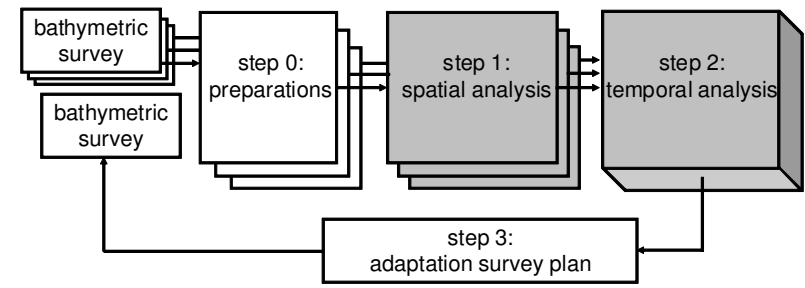

Figure 4.3: Overview of the procedure to analyze a series of surveys. The method described here consists of the two shaded steps.

tern variation can be identified as the $x$-direction of the grid, perpendicular to the direction of along-crest uniformity, which is the $y$-direction of the grid. We work with the relative sand wave parameters, with respect to their values $A_{\text {ref }}$ and $\xi_{\text {ref }}$ for a reference survey: amplitude factor $A^{\prime}=A / A_{\text {ref }}$ and migration $\xi^{\prime}=\xi-\xi_{\text {ref }}$. To determine the dominant wavelength $L^{(\mathrm{d})}$ of the sand wave extension, the test quotient becomes a function of wavelength: $q=q(L)$. Consequently, a wavelength search interval $\left[L_{\min }, L_{\max }\right]$ needs to be defined, based on an impression of the pattern. Subsequently, the best fitting wavelength is obtained by maximizing $q(L)$. If both spatial extensions are accepted, the morphological characterization $m_{s}(x, y)$ of the sea floor during survey $s$ becomes:

$$
m_{s}(x, y)=d_{s}^{(\mathrm{b})}+\psi_{s}^{(\mathrm{x})} x+\psi_{s}^{(\mathrm{y})} y-A_{s} \cos \left(2 \pi\left(x-\xi_{s}\right) / L^{(\mathrm{d})}\right) .
$$

The estimated depth values of a characterization are given in a vector $\underline{\mathbf{m}}$.

The dynamic extensions (step 2) define a linear parameter trend in all surveys, and outlying parameters for survey $s$, for each $s=1, \cdots, S$. For a parameter $u$, we consider the following extensions in time with respect to $u_{\text {ref }}$ at time $t_{\text {ref: }}$

1. a linear trend $\dot{u}$ of the parameter in time:

$$
u(t)=u_{\mathrm{ref}}+\dot{u}\left(t_{s}-t_{\mathrm{ref}}\right) .
$$

2. outlying parameter $\Delta u_{s}$ for survey $s$ :

$$
u(t)=\left\{\begin{array}{ll}
u_{\mathrm{ref}} & \left(\text { if } t \neq t_{s}\right) \\
u_{\mathrm{ref}}+\Delta u_{s} & \left(\text { if } t=t_{s}\right)
\end{array} ;\right.
$$

Because the two parameters $\psi^{(\mathrm{x})}$ and $\psi^{(\mathrm{y})}$, and the two parameters $A$ and $\xi$ are mutually correlated, an extension contains the two bed slope parameters, or the two sand wave parameters. First, these two trend extensions are tested. Depending on the values of the test quotients, the trend extensions are added to 
the characterization. After that the $2 S$ outlier extensions are tested, to account for nonlinear behaviour.

The levels of significance $\alpha$ specify the probability that an extension to a representation is incorrectly selected. The criteria in Chapter 3 define values for $\alpha$, based on criteria for the selection of extensions for one-dimensional analyses per grid line. Here, the levels of significance differ, as we set them for criteria for a two-dimensional analyses of a full grid. The criteria are as in Chapter 3, except for the grid used, which has a size of $1000 \times 1000 \mathrm{~m}$, and the allowed minimal detectable biases are set at $0.1 \mathrm{~m}$. (These are the values of the parameters that are allowed to remain undetected with a specified probability.) This small value illustrates that it is critical to detect dynamics at the scale of the full grid. The resulting levels of significance are $5 \%, 7 \%$ and $5 \%$ for the spatial extensions, the trend extensions and the outlier extensions, respectively. We also use these levels of significance for zero-dimensional analysis.

\section{Morphological residuals}

In our procedure, the iterative addition of extensions continues until all remaining extensions have a test quotient smaller than one. If the characterization fits the series of surveys well enough, from a statistical point of view, the final parameter values are calculated, as well as the associated uncertainties. The overall test is used for this decision, with its overall test quotient $q^{(o)}$ smaller than one to pass the test.

However, if the differences between the characterization and the measured sea floor are larger than can be explained by the uncertainty of the depth measurements, the characterization is insufficient. The differences are called the residuals, which contain measurement errors and residual variations, like asymmetries and bifurcations in the sand wave pattern, and small-scale rhythmic features like mega-ripples. Instead of defining additional parameters, which complicate the characterization, we assume that the residual variation is a random function, and include additional morphological variance and covariance components in the uncertainty, using Least Squares Variant Component Estimation (LSVCE) [Teunissen and Amiri-Simkooei, 2008].

\section{Final estimation}

The LSVCE-procedure is followed for both the steps explained above. The variances and covariances, describing the dispersion, that are calculated in step 1 are used in step 2. An increased dispersion during step 1 therefore has consequences in step 2. In the first place, it is less likely that an extension will be accepted in that step. Also, it means that the resulting dynamic parameters will be estimated less accurately. At the end of step 2, the second LSVCE procedure only influences the uncertainty of the final parameter estimates.

We denote the additional variance and covariance for morphology (step 1) $\underline{\sigma}^{(\mathrm{m}) 2}$ and $\underline{c}^{(\mathrm{m})}(h)$, respectively. The additional variance and covariance for temporal change (step 2) are denoted $\underline{\sigma}^{(\mathrm{t}) 2}$ and $\underline{c}^{(\mathrm{t})}(h)$. The final variance and 
covariance, given in $\underline{\mathbf{C}}_{d}$, consists of the initial covariance matrix $\underline{\mathbf{C}}_{d}^{(\mathrm{e})}$ and, if the overall test of that step was rejected, of $\underline{\mathbf{C}}_{d}^{(\mathrm{m})}$ and $\underline{\mathbf{C}}_{d}^{(\mathrm{t})}$. After the LSVCEprocedure, the final estimates of a step are calculated using a Best Linear Unbiased Estimation (BLUE) .

\subsubsection{Bathymetric data}

Five available surveys of the Maas West anchorage area, measured between 1994 and 2006, were used in a deformation analysis. The 2006 survey deployed a Multi-Beam Echo Sounder (MBES), the other surveys a Single-Beam Echo Sounder (SBES). Details on the surveys are given in Appendix 4.A.

The direction of the sand wave pattern, perpendicular to the crests, is approximately Northeast/Southwest for all subareas (Figure 4.4a). This direction was determined using the DIGIPOL method [Van Munster et al., 1995; RIKZ, 1997; De Koning, 2007], which calculates variability values $\underline{v}(\theta)$ for a series of directions $\theta$. The DIGIPOL-quotient between the maximum value of $\underline{v}$ for the direction of highest variability $\underline{\theta}^{(\mathrm{x})}$ and the minimum value of $\underline{v}$ for the direction of lowest variability $\underline{\theta}^{(\mathrm{y})}$ determines the strength of the preference for that direction. Figure $4.4 \mathrm{~b}$ shows the spatial variation per subarea by its 95 th percentile, around a fitted sloping plane. The Northwestern subareas seem to have the largest pattern amplitudes. This Figure also shows the bed level of each subarea, resulting from step 2.

The sea floor at the anchorage area has a rhythmic pattern of 200 to $400 \mathrm{~m}$ length, which are the limits of the wavelength search interval for the detection of sand waves. This is visible in the $20 \mathrm{~m}$ depth line of Figure 4.1. Shorefaceconnected ridges with a wavelength of several kilometres can be identified as well, in an approximately perpendicular direction.

Since these larger patterns are not the focus of this study, we restrict the size of the subareas to about a square kilometre. In this way, the dynamics on these larger scales will be reflected by a change in detected bed level or bed slope. A grid spacing of $50 \mathrm{~m}$ is sufficient to capture a sand wave pattern of $200 \mathrm{~m}$ length. This spacing allows for a grid size corresponding to the subarea size on an ordinary computer, which gives us eighteen subareas, denoted A to $\mathrm{R}$ (Figure 4.1).

\subsubsection{Error modeling}

The error $\underline{e}(x, y, t)$ at a position $\left(x_{p}, y_{p}\right)$ and during survey $s$ is denoted $\underline{e}_{p, s}$. It is the sum of several error sources:

$$
\underline{e}_{p, s}=\underline{e}_{p, s}^{(\mathrm{v})}+\underline{e}_{p, s}^{(\mathrm{h})}+\underline{e}_{p, s}^{(\mathrm{k})} .
$$

These sources are:

1. the combined influence of all processes involved in depth measurement $\underline{e}_{p, s}^{(\mathrm{v})}$;

2. the influence of position error $\underline{e}_{p, s}^{(\mathrm{h})}$; 

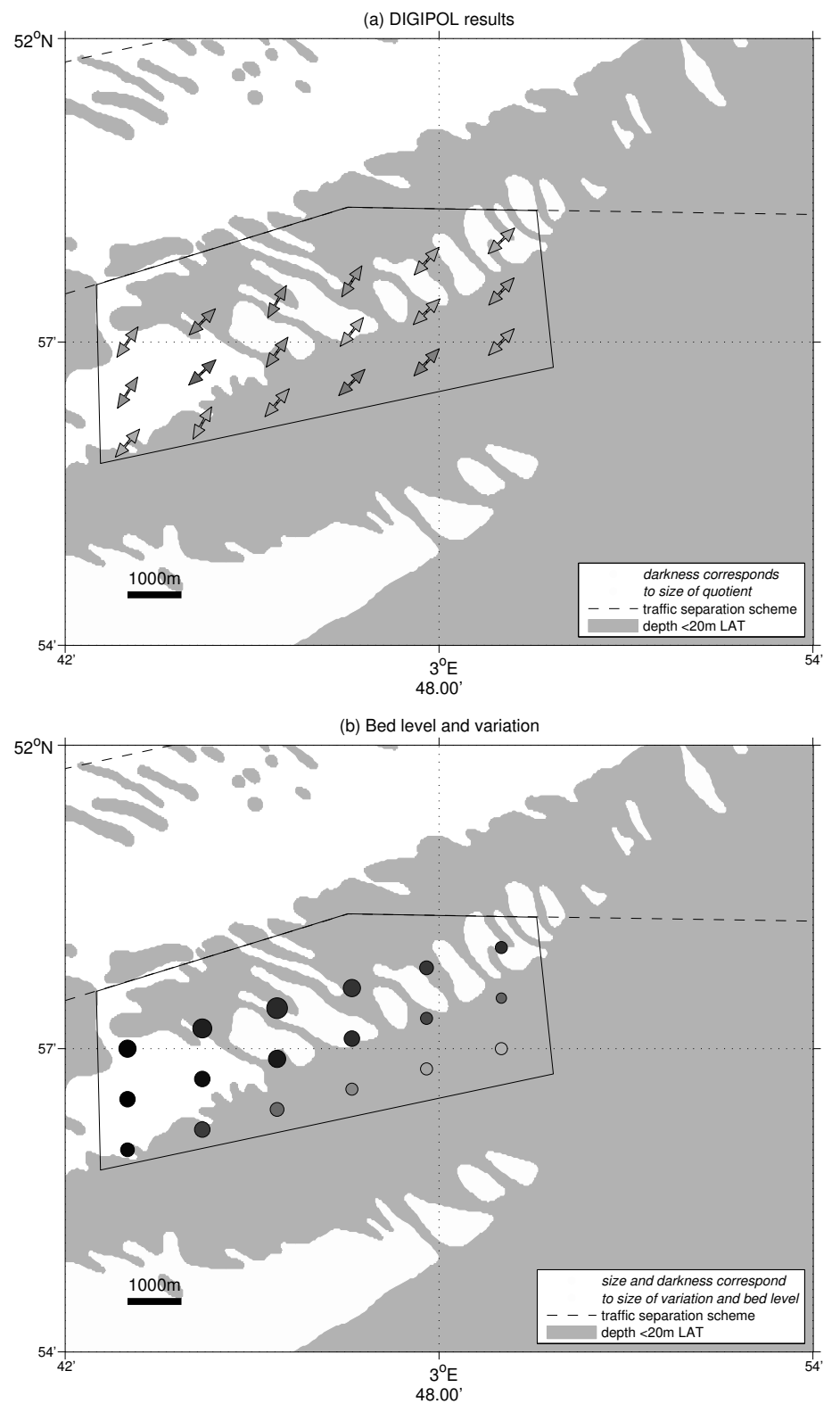

Figure 4.4: Spatial characteristics of the December 1994 survey: (a) DIGIPOL directions $\underline{\theta}^{(\mathrm{x})}$ and quotients $\max (\underline{v}) / \min (\underline{v})$ (quotients between 2.2 and 4.0, darker is larger); (b) bed level $\underline{d}^{(\mathrm{b})}$ (between 16 and $22 \mathrm{~m}$, shown by the shade of the circles, darker is deeper) and 95 th percentiles of the spatial variation $1.96 \underline{\sigma}_{d}^{(\mathrm{s})}$ (between 0.95 and $2.6 \mathrm{~m}$, shown by the size of the circles, larger is more variation). 
3. the potential influence of interpolation $\underline{e}_{p, s}^{(\mathrm{k})}$, using e.g. Kriging.

The largest contribution to the error $\underline{e}_{p, s}^{(\mathrm{v})}$ of depth measurement in the Southern North Sea outside the coastal zone is the water level reduction. It is common practice to reduce depth measurements to a low water surface, nowadays Lowest Astronomical Tide (LAT). The actual water level above this reduction surface is determined as indicated in Appendix 4.A.

The position error $\underline{e}_{p, s}^{(\mathrm{h})}$ consists of two components. The first one is position measurement, and the second one the binning error, for the assignment of depth measurements to the center of the archiving bin in which they fall. Nowadays, positioning is done by differential GPS, but for older surveys a system known as Hyperfix was used, see Appendix 4.A. The slopes of the sea floor on the BNLCS are so small that the effect of the binning error is negligible.

The interpolation error $\underline{e}_{p, s}^{(\mathrm{k})}$ is absent for MBES surveys, as they have overlapping swaths of depth measurements between tracks. Kriging [Chilès and Delfiner, 1999; Isaaks and Srivastava, 1989] is used for the interpolation of SBES surveys, quantifying the interpolation error as additional Kriging variance. On the other hand, all SBES measurements are done in vertical direction, which makes them more accurate than the MBES measurements that are done up to wide angles with the vertical, introducing a variety of errors. The quantification of the errors by variances $\sigma^{(\mathrm{e}) 2}$ and covariance functions $c^{(\mathrm{e})}(\mathbf{h})$ is done in Appendix 4.B.

\subsection{Results of the deformation analysis}

\subsubsection{General results for the subareas}

The procedure detects fifteen subareas that are regular enough to be characterized using the sand wave extension. Two examples are given in Figure 4.5. Details of all detected sand waves of step 1 are given in Figure 4.6. The amplitudes of the estimated sine functions are smaller than the spatial variations (Figure 4.4), because of e.g. the asymmetry of the waves, or variations in the crest direction. However, the larger amplitudes in the Northwest correlate with the larger spatial variations in the Northwest. For all subareas, the overall test of step 1 is rejected. Therefore, additional morphological variance is added to the uncertainty of the depth data.

The results of step 2 are given in Figure 4.7 and Table 4.1. Sand wave dynamics are found for eleven subareas. All those sand wave dynamics are linear trends, and no outliers were found for those trends. The trends were detected because of a constant migration rate, see Table 4.1. The average migration rate is $4.2 \mathrm{~m} / \mathrm{yr}$. The growth of the amplitudes is not significant, as the estimates are much smaller than the $95 \%$ confidence limits.

No migration was detected for four of the fifteen subareas with a sand wave, because in those subareas the LSVCE procedure in step 1 has added too much morphological variance to the uncertainty of the depth data. The consequence of a larger uncertainty is a reduction of the test quotients $\underline{q}$. For those four subareas, the test quotients were reduced to a value below one. 
(a) depth

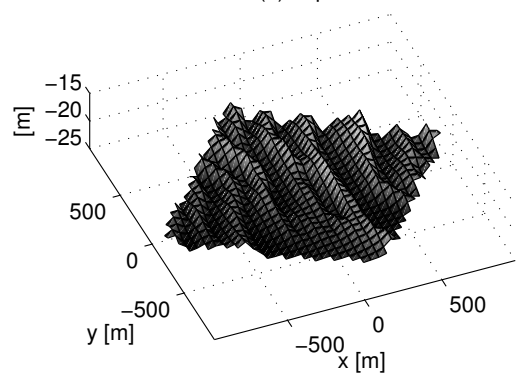

(c) depth

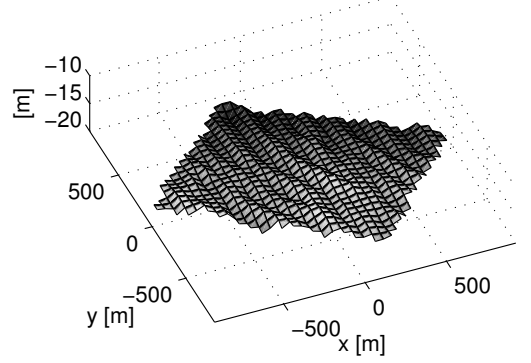

(b) characterization

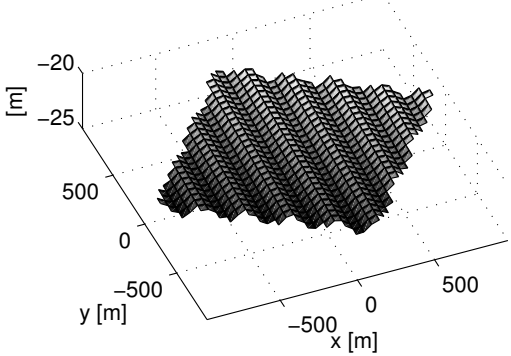

(d) characterization

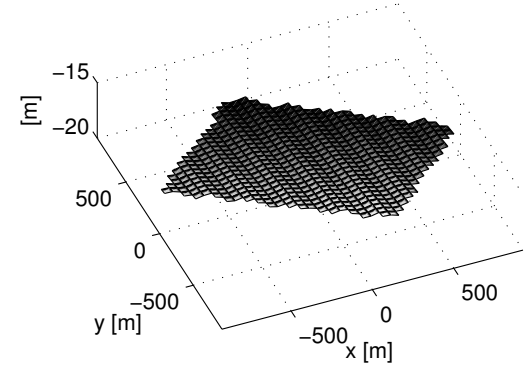

Figure 4.5: Depth values below Lowest Astronomical Tide and characterization for subareas H and Q: (a) gridded depth values for subarea H; (b) characterization after step 1 for subarea H; (c) gridded depth values for subarea Q; (d) characterization after step 1 for subarea $Q$. All values are averages over the five surveys. Note that the scales of the vertical axes differ from each other.

An overview of the pattern of all subareas where migration was found is given in Figure 4.7, ordered from a small to a large migration rate. Some of the wave crests are absent in subareas $\mathrm{F}$ and $\mathrm{K}$, which would result in a dominant wavelength that is larger than the upper boundary of the specified search interval. In spite of that, another maximum $\underline{q}(L)$ was detected within the interval. The disappearing crests cause the $1 \mathrm{~km}$-scale pattern visible in the $20 \mathrm{~m}$ depth line of Figure 4.1. A further observation of Figure 4.6 is that the wavelength seems to decrease with an increasing migration rate. This will be studied in more detail in Section 4.4.1.

The uncertainty of the migration rate estimates depend on the added morphological variance, and thereby on the regularity of the pattern. This is illustrated by subarea $\mathrm{H}$, which has both the most accurate migration rate and the largest DIGIPOL-quotient (Figure 4.4). Subarea H also has a relatively large amplitude (Figure 4.6), in spite of its relatively small spatial variation (Figure 4.4). The depth values and characterization of subarea $\mathrm{H}$ are given in Figure 4.5a. This subarea is studied in detail in Section 4.3.2. 

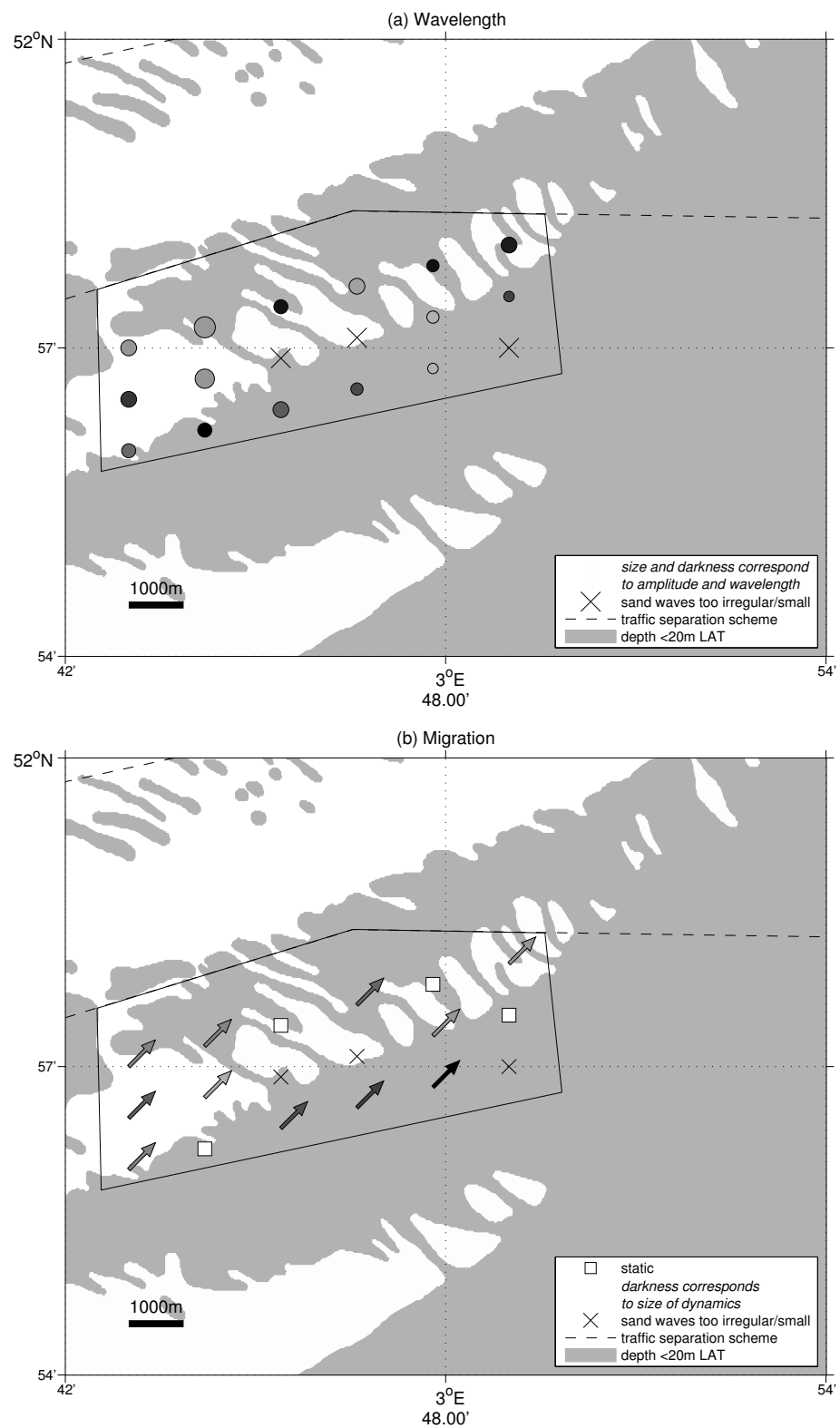

Figure 4.6: Results for the sand wave pattern: (a) step 1, detected dominant wavelengths $\underline{L}^{(\mathrm{d})}$ (208 to $360 \mathrm{~m}$, shown by shade of the circles, darker is larger wavelength) and their fitted amplitudes $\underline{A}$ ( 0.1 to $0.5 \mathrm{~m}$, shown by size of the circles, larger is larger amplitudes); (b) step 2, migration (2.4 to $7.5 \mathrm{~m} / \mathrm{yr}$, darker is larger migration rate). 
Table 4.1: Linear trends for two-dimensional sand wave dynamics. The estimates and uncertainties at a 95\% confidence level are given as estimate \pm uncertainty. A-F: Northern; G-L: Central; M-R: Southern.

\begin{tabular}{cc}
\hline \hline subarea & growth rate $\underline{\dot{A}}^{\prime}$ migration rate $\dot{\xi}^{\prime}$ \\
\hline $\mathrm{A}$ & $+1.6 \pm 9.1 \% / \mathrm{yr}+3.7 \pm 3.1 \mathrm{~m} / \mathrm{yr}$ \\
$\mathrm{B}$ & $+0.1 \pm 6.3 \% / \mathrm{yr}+3.6 \pm 2.3 \mathrm{~m} / \mathrm{yr}$ \\
$\mathrm{C}$ & no sand wave trend detected \\
$\mathrm{D}$ & $-1.1 \pm 8.1 \% / \mathrm{yr}+4.5 \pm 3.1 \mathrm{~m} / \mathrm{yr}$ \\
$\mathrm{E}$ & no sand wave trend detected \\
$\mathrm{F}$ & $+1.5 \pm 5.3 \% / \mathrm{yr}+3.1 \pm 2.6 \mathrm{~m} / \mathrm{yr}$ \\
\hline $\mathrm{G}$ & $-1.3 \pm 6.2 \% / \mathrm{yr}+4.7 \pm 3.3 \mathrm{~m} / \mathrm{yr}$ \\
$\mathrm{H}$ & $+0.3 \pm 4.6 \% / \mathrm{yr}+2.4 \pm 1.6 \mathrm{~m} / \mathrm{yr}$ \\
$\mathrm{I}$ & no sand wave pattern detected \\
$\mathrm{J}$ & no sand wave pattern detected \\
$\mathrm{K}$ & $+3.5 \pm 8.0 \% / \mathrm{yr}+3.1 \pm 2.2 \mathrm{~m} / \mathrm{yr}$ \\
$\mathrm{L}$ & no sand wave trend detected \\
\hline $\mathrm{M}$ & $+1.5 \pm 7.3 \% / \mathrm{yr}+3.6 \pm 2.9 \mathrm{~m} / \mathrm{yr}$ \\
$\mathrm{N}$ & no sand wave trend detected \\
$\mathrm{O}$ & $+0.8 \pm 6.4 \% / \mathrm{yr}+5.1 \pm 2.7 \mathrm{~m} / \mathrm{yr}$ \\
$\mathrm{P}$ & $+2.3 \pm 9.8 \% / \mathrm{yr}+5.6 \pm 4.2 \mathrm{~m} / \mathrm{yr}$ \\
$\mathrm{Q}$ & $+2.5 \pm 6.5 \% / \mathrm{yr}+7.5 \pm 1.9 \mathrm{~m} / \mathrm{yr}$ \\
$\mathrm{R}$ & no sand wave pattern detected \\
\hline \hline
\end{tabular}

The Southeastern subareas migrate faster than average: the largest migration rate found is $7.5 \mathrm{~m} / \mathrm{yr}$, for subarea $\mathrm{Q}$. This shallow subarea has a small dominant wavelength and a small amplitude (Figures 4.4 and 4.6). The small amplitude is a true reflection of the small spatial variation in depth (Figure 4.4), and not caused by high irregularity of the pattern, as its DIGIPOL-quotient is about average (Figure 4.4). The depth values and characterization of subarea $\mathrm{Q}$ are given in Figure 4.5b.

The effect of migrating sand waves on depth is a decreasing depth at the side of the wave crests to which they migrate, continuing until the crest has passed. The associated danger of this decrease to shipping is shown in Figure 4.8, using zero-dimensional analyses per grid node. For such an analysis, the only spatial parameter involved is bed level. Often, linear trends are detected for those nodes that are located on a Northeastern slope of a sand wave crest. These trends can have a size of up to $0.5 \mathrm{~m} / \mathrm{yr}$ upward for the anchorage area.

\subsubsection{Detailed results for subarea $\mathbf{H}$}

The results of subarea $\mathrm{H}$ are the most accurate, and therefore described in more detail. Figure 4.9 shows the resulting sand wave parameters of step 1 using the white circles. Both a slope in the plane and a sand wave pattern were 

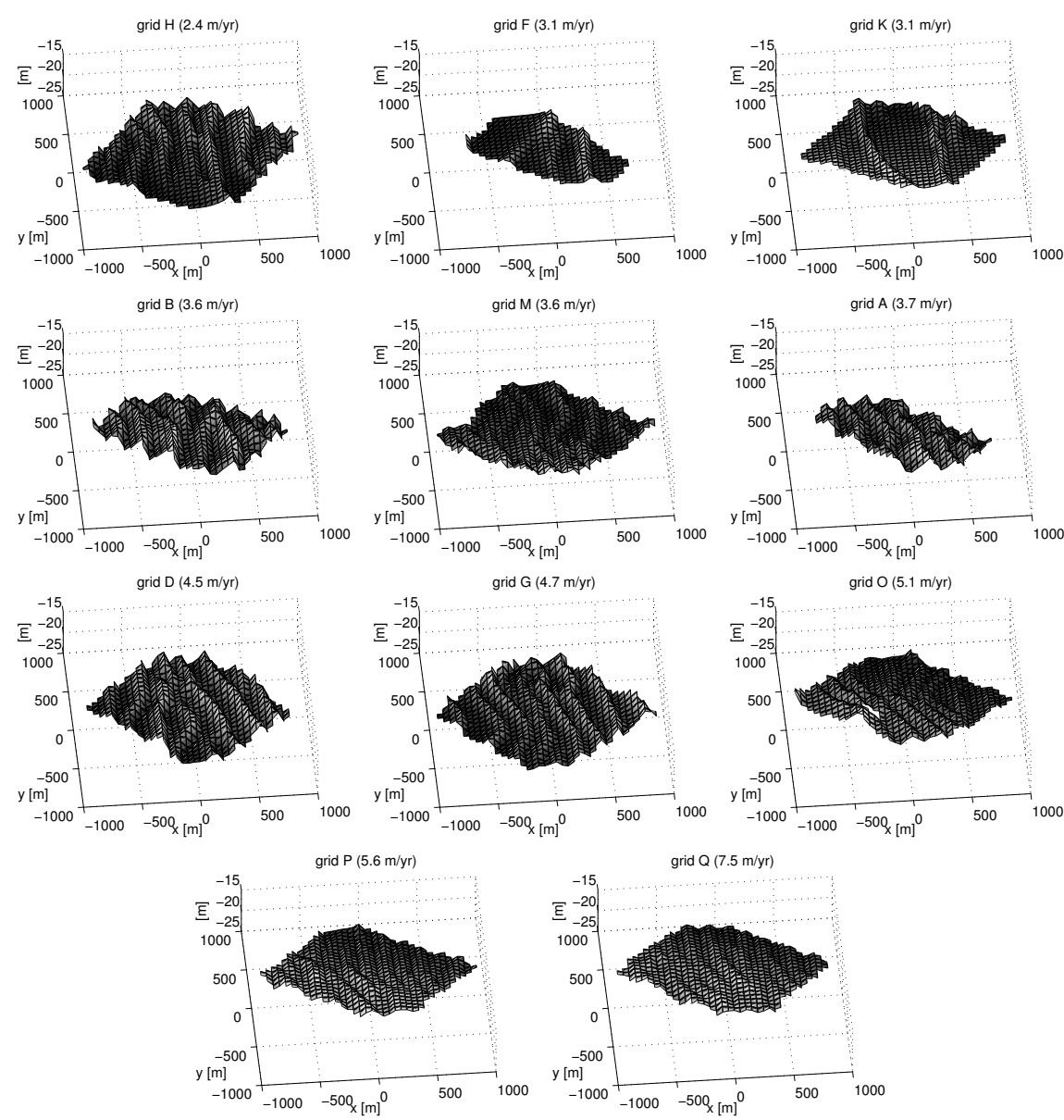

Figure 4.7: Depth values and migration rate for all subareas where migration is detected, ordered from small migration rate estimates to large estimates.

detected, as the test quotients $\underline{q}$ for both these extensions are larger than one (Figure 4.10). Therefore, five parameters per survey are used.

The planar results are static, and the sand wave results show the trend. The black circles in Figure 4.9 show the sand wave results after step 2. The error bars and the crosses indicate the $95 \%$ confidence intervals of the results of step 1 and step 2, respectively. In step 1, the five selected spatial parameters were used to describe each survey individually, so there are 25 parameters. In step 2 , the results are described by seven parameters: three planar static parameters, two sand wave parameters for the reference survey (the first), and two sand wave trend parameters. 


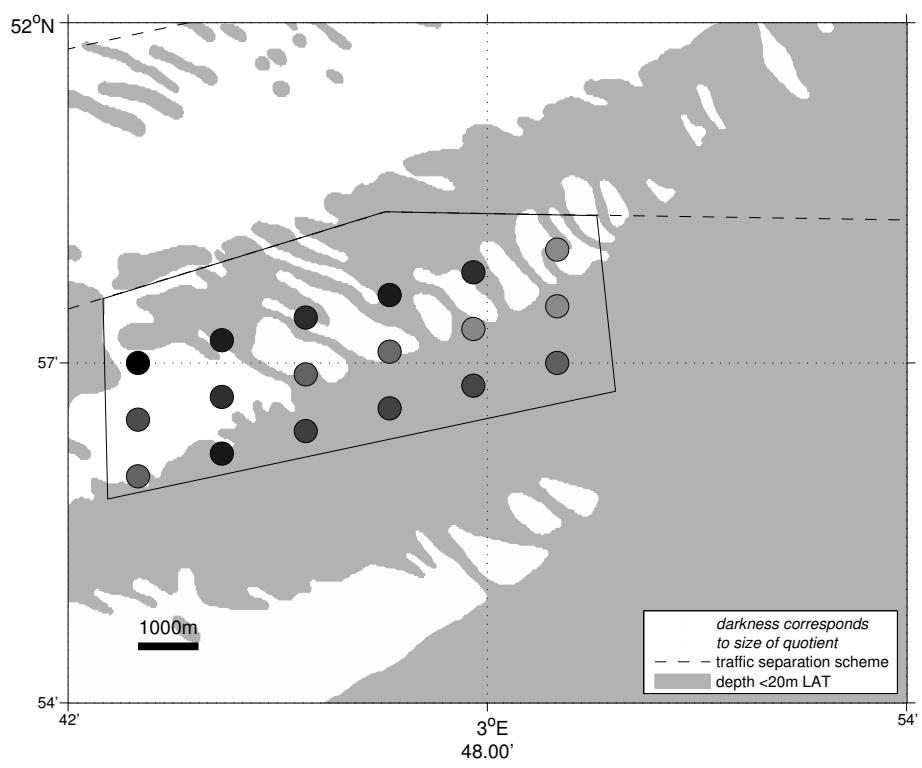

Figure 4.8: Maximum upward linear trends in bed level $\underline{\dot{d}}^{(b)}$, detected by the zerodimensional analyses (darker is larger trend).

The differences between the planar estimates of step 1 are so small that the initial static characterization is accepted. The crest positions of step 1 clearly change linearly with time, which is recognized as migration during step 2. In general, Figure 4.9a shows that the amplitude factors in step 2 are smaller than the amplitude factors obtained in step 1. As there are fewer parameters, it is harder to adapt the step 2 representation to the actual situation, causing the sand wave pattern to fit less well. The worse fit becomes apparent by the smaller amplitude of the pattern. It is clear that the confidence intervals of step 2 are smaller than those of step 1 . There are less parameters in step 2, while the number of depth values at the input side stays the same. As the amount of data per parameter increases, the results become more accurate.

The results of a zero-dimensional analysis confirms the observed migration (Figure 4.11). At the side of the crests that lies in positive $x$-direction downward trends are found, and at the side in negative $x$-direction, there are upward trends. The largest upward trend is used to create Figure 4.8. Also, occasional outliers are detected at the crests. These are the positions were an upward trend is reversed into a downward trend. The outliers are needed here to account for this nonlinear depth change. In the troughs, such an effect is not visible, because they are less pronounced. 

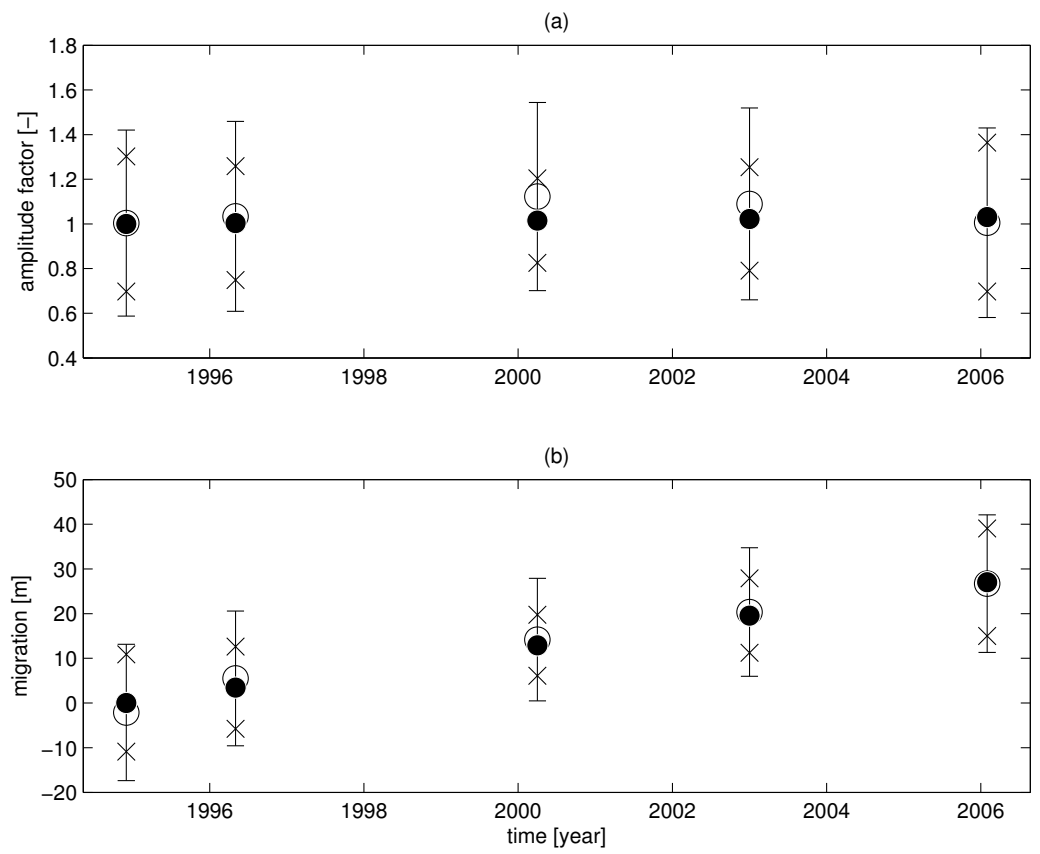

Figure 4.9: Sand wave results for subarea H, for a dominant wavelength $\underline{L}^{(\mathrm{d})}$ of 231 $\mathrm{m}$ and a reference amplitude $A_{\text {ref }}$ of $0.38 \mathrm{~m}$ : (a) amplitude factor $\underline{A^{\prime}}$; (b) migration $\xi^{\prime}$. The white circles are the results after step 1, and the black circles indicate a linear trend, detected in step 2. The variations that step 1 show are approximated by the step 2 results, which are exactly on a sloping straight line. The sand wave trend of step 2 is estimated as (a) a yearly growth rate $\underline{\dot{A}}^{\prime}$ for the amplitude factor of 0.003 (or $0.3 \% / \mathrm{yr}$ ), and (b) a migration rate $\underline{\xi}^{\prime}$ of $2.4 \mathrm{~m} / \mathrm{yr}$. The vertical error bars and the crosses indicate the $95 \%$ confidence intervals of the results of step 1 and step 2 , respectively.

\subsection{Discussion of results}

Correlation between migration of measured sand waves in the Southern North Sea and other morphological parameters has been studied before. To validate the results of our method, we make comparisons with two earlier papers on observed sand wave migration in the Southern North Sea, and with an idealized process-based model. Knaapen [2005] finds a correlation between observed migration rates and a predictor for migration rate, which is based on three parameters for sand wave shape, for migration rates up to $10 \mathrm{~m} / \mathrm{yr}$. Van Dijk and Kleinhans [2005] report for sites closer to the shore that observed migration rates up to $20 \mathrm{~m} / \mathrm{yr}$ exist, depending on depth. These correlations are checked against our analysis in Section 4.4.1. Idealized process-based models for tidal sand wave dynamics confirm detected observed rates for the Southern North 


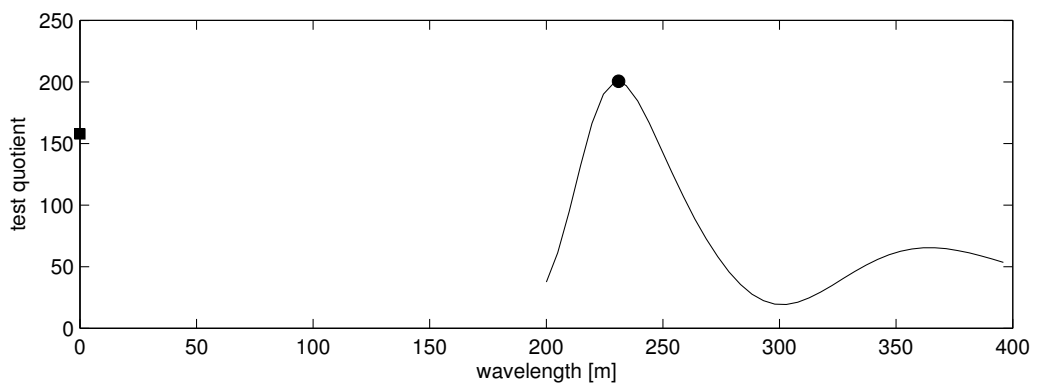

Figure 4.10: Test quotients $q$ for step 1 of subarea $H$. The square at the vertical axis represents the test quotient for the slopes, and the curve the test quotients for sand waves as a function of wavelength. The circle represents the maximum test quotient, which defines the dominant wavelength $\underline{L}^{(\mathrm{d})}$ as $231 \mathrm{~m}$.

Sea [Németh et al., 2007; Besio et al., 2008]. We compare our results with the output of such a model in Section 4.4.2.

\subsubsection{Correlations with spatial parameters}

To test the depth dependency reported by Van Dijk and Kleinhans [2005], we use the estimates and uncertainties for the migration rates $\underline{\xi}^{\prime}$ in a linear regression analysis with bed level $\underline{d}^{(\mathrm{b})}$. This leads to:

$$
\underline{\dot{\xi}}^{\prime}=a_{d} \underline{d}^{(\mathrm{b})}+b_{d} .
$$

Knaapen [2005] calculates a predictor $\dot{\xi}^{(\mathrm{p})}$ for the migration rate in the direction of the steep side as

$$
\dot{\xi}^{(\mathrm{p})}=c L^{(\mathrm{d}) 2} G^{2} / H,
$$

with sand wave height $H$ and dimensionless horizontal asymmetry (also leestoss asymmetry and skewness) $G$. The value of the calibration constant $c$ is $9.78 \cdot 10^{-4} \mathrm{yr}^{-1}$. Our results do not include sand wave height, but the related spatial variation $1.96 \sigma_{d}^{(\mathrm{s})}$ at a $95 \%$ confidence level is available. The spatial variation is assumed to be half the wave height, $H=3.92 \underline{\sigma}_{d}^{(\mathrm{s})}$.

Horizontal asymmetry is defined by Knaapen [2005] as $\left(h_{x}^{-}-h_{x}^{+}\right) / L^{(\mathrm{d})}$ with $h_{x}^{-}$the distance from crest to trough in negative direction of the $x$-axis and $h_{x}^{+}$the distance from crest to trough in its positive direction. Our results do not include such a dimensionless parameter for horizontal asymmetry. However, differences from a sine shaped sand wave pattern are quantified in the morphological variance $\underline{\sigma}^{(\mathrm{m}) 2}$, if a sand wave pattern is accepted during step 1 . These differences include horizontal asymmetries. Assuming that all subareas have sand waves with equal vertical asymmetry (also crest-trough asymmetry and peakedness) and equal crest length, we could use these variances to indicate 


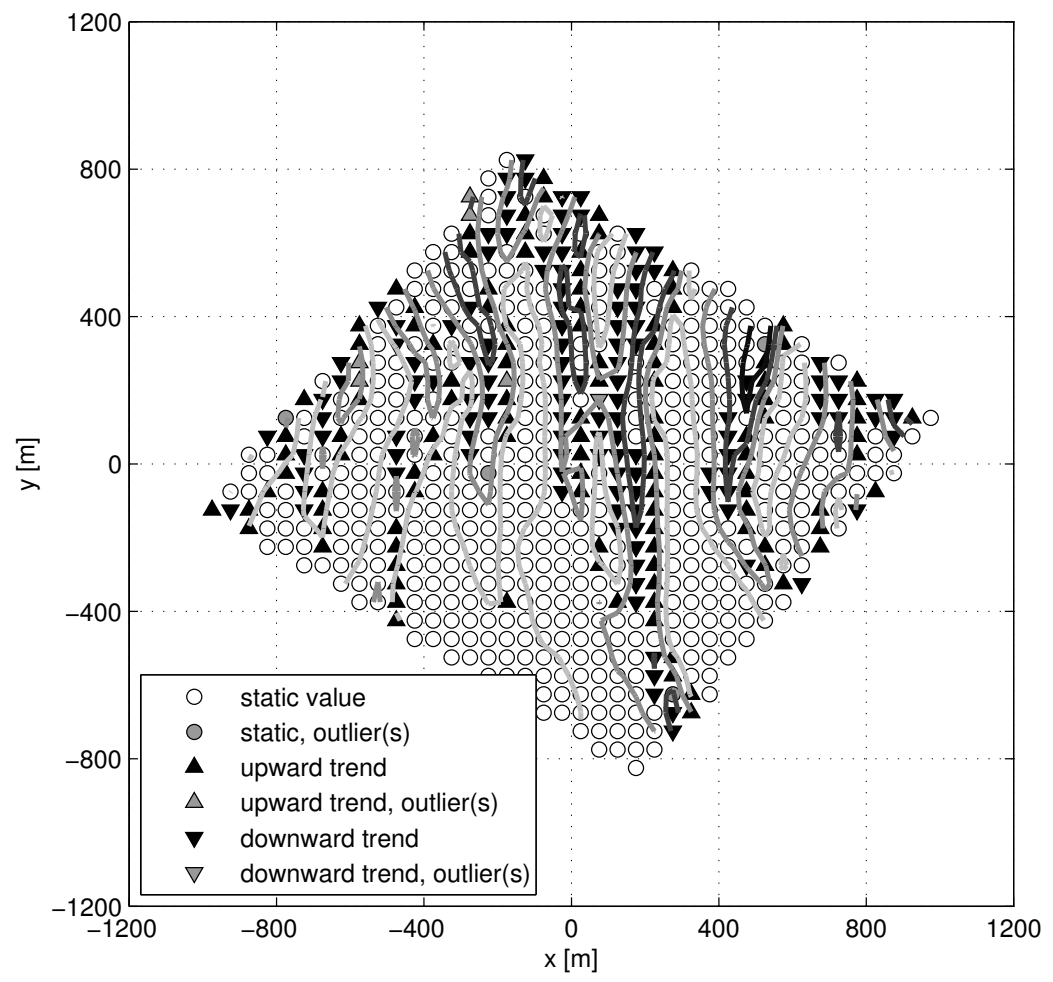

Figure 4.11: Zero-dimensional dynamics per grid node, detected at the individual grid nodes of subarea $\mathrm{H}$, and lines of equal depth to show the sand wave structure, also shown in the first graph of Figure 4.7. The trends $\underline{\dot{d}}^{(\mathrm{b})}$ have values up to 0.35 $\mathrm{m} / \mathrm{yr}$, and the outliers $\Delta \underline{d}_{s}^{(\mathrm{b})}$ up to $1.8 \mathrm{~m}$.

asymmetry. Taking the square root and dividing by the spatial variation makes the morphological variance dimensionless, and we assume $G=\underline{\sigma}^{(\mathrm{m})} /\left(1.96 \underline{\sigma}_{d}^{(\mathrm{s})}\right)$.

These two assumptions give us the adapted predictor as:

$$
\underline{\dot{\xi}}^{(\mathrm{p})}=c^{(\mathrm{a})} \underline{L}^{(\mathrm{d}) 2} \underline{\sigma}^{(\mathrm{m}) 2} / \underline{\sigma}_{d}^{(\mathrm{s}) 3} .
$$

The calibration constant $c^{(a)}$ is adapted for the conversion factors $1 / 1.96$ and 3.92 of $G$ and $H$ respectively. Its value is $6.49 \cdot 10^{-5} \mathrm{yr}^{-1}$. This new expression enables us to calculate the linear correlation with the estimated migration rate:

$$
\underline{\dot{\xi}}^{\prime}=a_{\xi} \dot{\dot{\xi}}^{(\mathrm{p})}+b_{\xi} .
$$


(a)

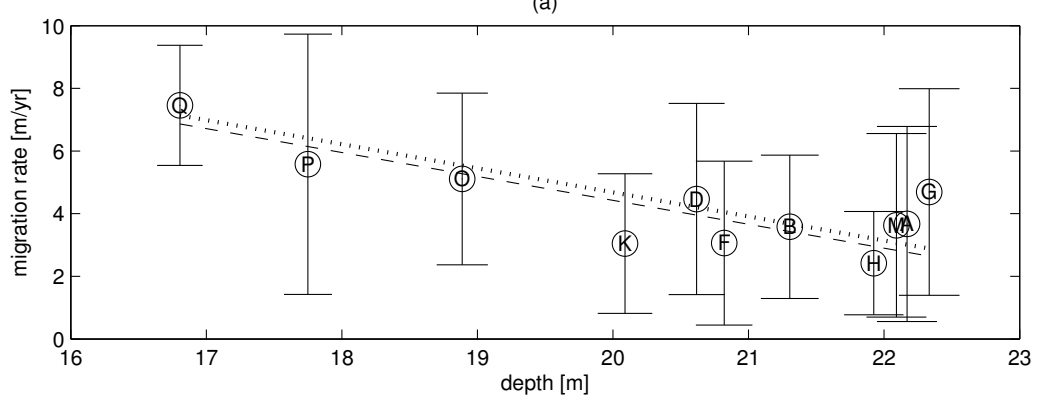

(b)

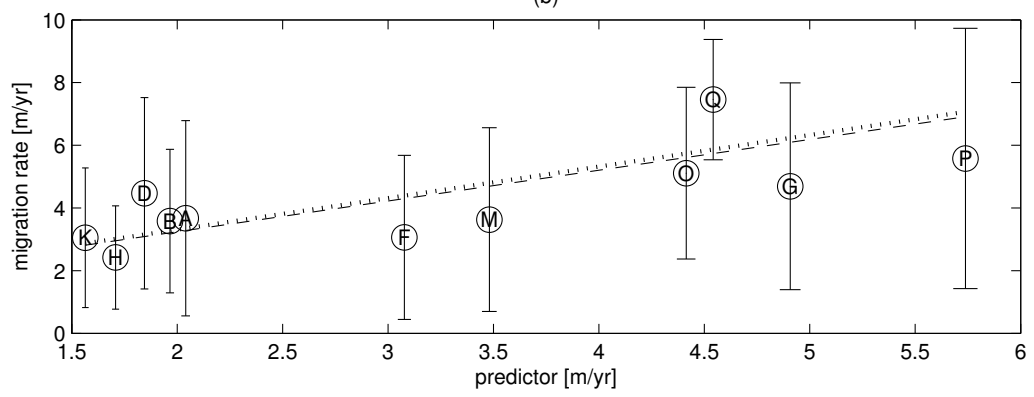

Figure 4.12: Estimates of linear correlations between migration rate $\underline{\xi}^{\prime}$ and (a) bed level $\underline{d}^{(\mathrm{b})}$ and (b) migration predictor $\dot{\xi}^{(\mathrm{p})}$, based on the subareas for which migration is detected. The dashed lines include all migrating subareas, and the dotted lines exclude the subareas $\mathrm{F}$ and $\mathrm{K}$.

The estimates for migration rate are plotted against bed level and the predictor in Figure 4.12, including the 95\% confidence intervals for migration rate. The resulting linear correlations are also given in Figure 4.12 and Table 4.2. Table 4.2 gives the correlation coefficients as well. We assume the bed level estimates are deterministic, because their 95\% confidence limits are very small: $0.07 \mathrm{~m}$, on average. The correlation coefficients reveal a strong correlation with both bed level and the predictor. As pointed out in Section 4.3.1, the sand wave patterns of subareas $\mathrm{F}$ and $\mathrm{K}$ are in fact not showing a wavelength in the specified range. We exclude the two subareas, and calculate the correlations again. The results are again given in Figure 4.12 and Table 4.2. The correlations and their coefficients hardly change, though.

The two papers and our results indicate that it is possible to predict migration rates based on morphological information of the sea floor. To see if such a correlation even is apparent from a visual inspection of the shape of the sea floor, we order the graphs of the measured depth values of each subarea for which migration is detected, from small migration rate estimates to large estimates (Figure 4.7). Most of the subareas show asymmetry, confirming the 
Table 4.2: Correlation estimates $\underline{a}, \underline{b}$ and their uncertainties expressed as $95 \%$ confidence limits, given as estimate \pm uncertainty, for migration rate $\underline{\xi}^{\prime}$ with bed level $\underline{d}^{\text {(b) }}$ and migration predictor $\underline{\dot{\xi}}^{(\mathrm{p})}$. These estimates are used in equations 4.5 and 4.8. Also, correlation coefficients $\underline{\rho}$ are given.

\begin{tabular}{|c|c|c|c|}
\hline & $\underline{a}$ & $\underline{b}$ & $\underline{\rho}$ \\
\hline \multicolumn{4}{|c|}{ including subareas $\mathrm{F}$ and $\mathrm{K}$ : } \\
\hline \multicolumn{4}{|c|}{$\underline{d}^{(\mathrm{b})}-0.76 \pm 0.40 \mathrm{yr}^{-1} 19.7=$} \\
\hline$\underline{\underline{\xi}}^{(\mathrm{p})}$ & $0.98 \pm 0.56$ & $1.3 \pm 1.8 \mathrm{~m} / \mathrm{yr}$ & +0.72 \\
\hline \multicolumn{4}{|c|}{ excluding subareas $\mathrm{F}$ and $\mathrm{K}$ : } \\
\hline$\dot{\xi}^{(\mathrm{p})}$ & $1.01 \pm 0.60$ & $1.3 \pm 2.0 \mathrm{~m} / \mathrm{yr}$ & +0.71 \\
\hline
\end{tabular}

predictive power of sand wave asymmetry for migration. The relation between migration rate on one hand and bed level, wavelength, and wave height on the other hand is not clear from Figure 4.7.

\subsubsection{Comparison with a sand wave model}

In this Section, we will compare the estimated migration rates with rates found using a process-based sand wave model. Using such an idealized process-based model, Hulscher [1996] showed that the formation of tidal sand waves can be explained as an inherent instability of a horizontal, sandy seabed subject to tidal flow. This linear stability analysis leads to a so-called fastest growing mode, i.e. preferred values of the wavelength $L^{(\mathrm{d})}$ and the growth rate. Later on, the physics of this model have been refined in various respects. For example, sand wave migration can be explained quantitatively as resulting from the specification of a residual current [Németh et al., 2002] or a higher harmonic to the tidal forcing [Besio et al., 2004].

Recently, Van den Berg and Van Damme [2005] extended the above approach into the finite amplitude regime, leading to a nonlinear model capable of describing tidal sand waves in morphodynamic equilibrium, see also Sterlini et al. [2009]. First, the model identifies the fastest growing mode. Next, the nonlinear evolution of this mode results in an equilibrium height, shape and migration rate.

The nonlinear sand wave model considers a seabed topography that varies in one horizontal dimension. The nonlinear shallow water equations describe the 2DV tidal flow pattern over the bed. The bed shear stress then controls the sediment transport, which is calculated with a simple power law including bed slope effects. Turbulence is accounted for using a constant eddy viscosity 
in combination with a partial slip condition at the seabed. This condition reads

$$
A_{\mathrm{v}} \frac{\mathrm{d} u_{\mathrm{h}}}{\mathrm{d} z}=S u_{\mathrm{h}}
$$

where $A_{\mathrm{v}}$ is the vertical eddy viscosity, $u_{\mathrm{h}}$ the flow velocity, and $S$ the slip parameter. For further details of the model setup, see Van den Berg and Van Damme [2005] and Sterlini et al. [2009].

In the following, we will compare the migration rates obtained from our deformation analysis with output of the nonlinear sand wave model introduced above. In our simulations, we consider a mean water depth of $20 \mathrm{~m}$, a seabed consisting of uniform sediment with a grain size of $250 \mu \mathrm{m}$, a constant vertical eddy viscosity of $0.03 \mathrm{~m}^{2} / \mathrm{s}$ and a slip parameter of $0.01 \mathrm{~m} / \mathrm{s}$. The mean water depth, depth-averaged flow velocity of the M2-tidal current, and grain size are in agreement with observations, while the vertical eddy viscosity and slip parameter are chosen according to Németh et al. [2002]. Following Németh et al. [2002], we introduce a residual current to induce sand wave migration. However, due to the uncertainty in the choice of a value for the local residual current, we consider three different values, which are realistic for the North Sea.

The three types of flow conditions are:

1. an M2-tidal current with a maximum depth-averaged flow velocity of 0.5 $\mathrm{m} / \mathrm{s}$;

2. an M2-tidal current with a maximum depth-averaged flow velocity of 0.5 $\mathrm{m} / \mathrm{s}$ plus a residual current of $0.05 \mathrm{~m} / \mathrm{s}$;

3. an M2-tidal current with a maximum depth-averaged flow velocity of 0.5 $\mathrm{m} / \mathrm{s}$ plus a residual current of $0.10 \mathrm{~m} / \mathrm{s}$.

The results are given in Table 4.3. The resulting values of the preferred wavelengths are in fair agreement with the observed wavelengths. As expected due to the symmetry in the type 1 flow conditions, there is no migration. The migration rates obtained with type 2 and 3 flow conditions are in the same order of magnitude as the results from the deformation analysis. This agreement is promising. However, we feel that a quantitatively more detailed comparison is not yet feasible, in view of the uncertainties in especially the model input.

\subsection{Conclusion}

The tidal sand waves in anchorage area Maas West show migration rates up to $7.5 \mathrm{~m} / \mathrm{yr}$, which make depth values decrease at one side of the crests, and thereby endanger navigation and anchoring. The migration rate is larger in the shallower subareas in the Southeast, while it also depends on the shape of the sand waves. This confirms the results of Van Dijk and Kleinhans [2005] and Knaapen [2005], and is in fair agreement with the results from idealized process-based models for tidal sand waves [Sterlini et al., 2009]. 
Table 4.3: Results of the process-based model of Sterlini et al. [2009] for the three types of flow conditions.

\begin{tabular}{cccc}
\hline \hline type & currents $u_{\mathrm{h}}:$ & \multicolumn{2}{c}{ wavelength migration rate } \\
& M2 tidal; M0 residual & $L^{(\mathrm{d})}$ & $\dot{\xi}^{\prime}$ \\
\hline 1 & $0.5 \mathrm{~m} / \mathrm{s} ; 0 \mathrm{~m} / \mathrm{s}$ & $300 \mathrm{~m}$ & $0 \mathrm{~m} / \mathrm{yr}$ \\
2 & $0.5 \mathrm{~m} / \mathrm{s} ; 0.05 \mathrm{~m} / \mathrm{s}$ & $270 \mathrm{~m}$ & $2 \mathrm{~m} / \mathrm{yr}$ \\
3 & $0.5 \mathrm{~m} / \mathrm{s} ; 0.10 \mathrm{~m} / \mathrm{s}$ & $270 \mathrm{~m}$ & $6 \mathrm{~m} / \mathrm{yr}$ \\
\hline \hline
\end{tabular}

Deformation analysis allows us to estimate linear correlations between morphology and migration rate, including the uncertainty of the estimates. This facilitates the comparison with observed migration rates in literature and processbased models. To ensure safe navigation and anchorage in the approach to the Port of Rotterdam, it is important to carefully set the resurvey frequency. The estimation of the size of the sea floor dynamics of this area, and the uncertainty of the estimates, is essential information thereto.

\section{Appendices}

\section{A Overview of used surveys}

All survey data used are given in WGS84 in combination with an UTM31projection. The data are reduced to Lowest Astronomical Tide. Only surveys are used for which the data are available on the highest resolution, i.e. each $5 \mathrm{~m}$ $\mathrm{x} 3 \mathrm{~m}$ archiving bin is filled, if possible. For SBES surveys the track distance is $50 \mathrm{~m}$, and for the MBES survey a full coverage of the sea floor at the bin level was achieved. All surveys are available in meters with two decimals, horizontally and vertically. Gross errors were removed from the surveys as part of the quality control procedure, after which each survey was approved by the commanding officer and Hydrographic Office of the RNLN.

In Table 4.4, the survey details are presented. Selective Availability (SA) is a deliberate degradation of the GPS signals. As differential GPS (DGPS) is used, the impact of SA is not large. The differential reference station used is either part of the IALA chain, or the VeriPos chain. The older surveys use the terrestrial Hyperfix system, which gives the same order of positioning uncertainty as DGPS with SA, i.e. in the order of $10 \mathrm{~m}$. DGPS without SA has an uncertainty of about 2.5 meters. The water level reduction method is either based on measurements from pressure gauges placed on the sea floor for the duration of the survey, or from permanent gauges in combination with the PreMo prediction model.

The track direction is decided by the commanding officer of the survey vessel, and can therefore vary between surveys. As a constant direction is not possible, due to e.g. traffic, the track direction is estimated from the data up to the 
Table 4.4: Overview of surveys, including survey track direction East of North $\theta^{(\mathrm{st})}$, and used survey systems for depth, horizontal reference and vertical reference.

\begin{tabular}{lrll}
\hline \hline survey ID & start of survey & $\theta^{\text {(st) }}$ & survey systems \\
\hline HY94067 & December 1994 & $40^{\circ}$ & SBES, Hyperfix, pressure gauges \\
HY96067 & May 1996 & $80^{\circ}$ & SBES, DGPS SA on, pressure gauges \\
HY00115 & April 2000 & $30^{\circ}$ & SBES, DGPS SA off, PreMo \\
HY02144 & January 2003 & $30^{\circ}$ & SBES, DGPS SA off, PreMo \\
HY06169 & February 2006 & $75^{\circ}$ & MBES, DGPS SA off, PreMo \\
\hline \hline
\end{tabular}

nearest five degrees. The first day of the month in which the survey started is used as the survey moment.

\section{B Quantification of measurement errors}

Errors $\underline{e}_{p, s}$ during survey $s$ are described by variances $\sigma_{p, s}^{(\mathrm{e}) 2}$ at each position $\left(x_{p}, y_{p}\right)$, and covariances $c_{p, q, s}^{(\mathrm{e})}$ between the positions $\mathbf{x}_{p}=\left(x_{p}, y_{p}\right)$ and $\mathbf{x}_{q}=$ $\left(x_{q}, y_{q}\right)$. Covariance $c_{p, p, s}^{(\mathrm{e})}$ equals variance $\sigma_{p, s}^{(\mathrm{e}) 2}$. The covariances between surveys are assumed to be zero. The common $95 \%$ confidence interval has its limits at $1.96 \sigma_{p, s}^{(\mathrm{e})}$.

\section{B.1 Covariance function of the error}

To describe the error covariances $c_{p, q, s}^{(\mathrm{e})}$, we assume second order stationarity, which means that the covariances only depend on position difference $\mathbf{h}$. An exception to this assumption is the variance at $h=0$. Following equation (4.4), the covariance function $c_{s}^{(\mathrm{e})}(\mathbf{h})$ of the measurement errors during survey $s$ is:

$$
c_{s}^{(\mathrm{e})}(\mathbf{h})=c_{s}^{(\mathrm{v})}(\mathbf{h})+c_{s}^{(\mathrm{h})}(\mathbf{h})+c_{s}^{(\mathrm{k})}(\mathbf{h})
$$

The Kriging error is assumed not to be correlated (Chapter 2), which means that its covariance function $c_{s}^{(\mathrm{k})}(\mathbf{h})$ is zero for distances $h>0$. The slopes of the sea floor of shallow seas are such that we neglect the effect of the two types of positioning errors, even in the presence of rhythmic patterns at various scales, which means that $c_{s}^{(\mathrm{h})}(\mathbf{h})$ equals zero for all distances $h \geq 0$. The application of LSVCE in step 1 will correct for this simplification, if necessary. The error covariance function is therefore assumed to equal the error covariance function $c_{s}^{(\mathrm{v})}(\mathbf{h})$ of the depth measurements.

We assume the uncertainty of the water level reduction has a $95 \%$ confidence limit of $0.2 \mathrm{~m}$, corresponding to a variance of $0.01 \mathrm{~m}^{2}$. The water level values, above the reduction surface, change relatively slowly, and are updated once every ten minutes, which means that the reduction component of the error variance is spatially correlated during the distance the ship travels in ten minutes, over the 
width of the track spacing of $50 \mathrm{~m}$. With a ship speed of $8 \mathrm{knots}$, or $15 \mathrm{~km} / \mathrm{h}$, the distance traveled is $2.5 \mathrm{~km}$ per ten minutes. From these surveying details, it follows that the error covariance is represented best as an anisotropic Gaussian function, with its highest covariance in the track direction, a maximum at the origin of $0.01 \mathrm{~m}^{2}$, and its inflection points at $2500 \mathrm{~m}$ and $50 \mathrm{~m}$ in along-track and across-track direction, respectively.

The variance appears as a discontinuity in this covariance function, at $h=0$, for which we do not assume second-order stationarity. Hence, the variance $\sigma_{p, s}^{(\mathrm{e}) 2}$ differs per depth value: the uncertainty of a depth measurement depends on depth itself [International Hydrographic Organization, 2008a].

\section{B.2 Variances of the SBES surveys}

The variances of the used Single-Beam Echo Sounder (SBES) surveys of the NZO-class survey vessels of the RNLN are modeled according to the components of Dorst [2004]. The surveys of these vessels that are used in this study are listed in Table 4.4. Assuming that all error sources follow Gaussian distributions, the variances associated with the measurement errors for the SBES surveys are the sum of all variances for the individual error sources listed in Table 4.5, i.e.:

$$
\sigma_{p, s}^{(\mathrm{v}) 2}=\sum_{i=1}^{10} \sigma_{i, p, s}^{(\mathrm{v}) 2}=1.4 \cdot 10^{-6} d_{p, s}^{2}+0.0114
$$

The influence of the error of the sound velocity profile measurements must be derived from the relation of sound velocity with depth measurement, which is $\underline{d}_{p, s}=\underline{v} t_{2 d} / 2$, with $\underline{v}$ the measured sound velocity and $t_{2 d}$ the two-way travel time. Consequently, the influence of the sound velocity profile error is $\sigma_{2}^{(\mathrm{v}) 2}=$ $\sigma_{v}^{2}\left(t_{2 d} / 2\right)^{2}$. Assuming an average sound velocity of $1500 \mathrm{~m} / \mathrm{s}$, the two-way travel time is $2 d_{p, s} / 1500 \mathrm{~s}$, and the relation between depth variance and sound velocity variance becomes $\sigma_{2, p, s}^{(\mathrm{v}) 2}=\sigma_{v}^{2} d_{p, s}^{2} / 1500^{2}$. The variance $\sigma_{v}^{2}$ of the sound velocity profile error is $1 \mathrm{~m}^{2} / \mathrm{s}^{2}$, for the used sound velocity profiler. The variance $\sigma_{v}^{2}$ is assumed constant, as sound velocity profiles are taken that often that their degradation in time is neglected.

During a bathymetric survey, the survey ship is constantly performing motions, measured and corrected for by a motion sensor. Roll and pitch are the rotations around the along-ship and across-ship axes, and heave is the highfrequent change in vertical ship position with respect to the water level. As surveying is only done during low sea states and the beam-width of an SBES is relatively wide, roll and pitch errors are neglected. Heave measurement on board the NZO-class vessels has a standard deviation of a tenth of the heave amplitude. As heave data is usually not available in the bathymetric archives, and because of the sea state limitation, this error in the heave correction is also neglected. It is also assumed that there is a clearly identifiable depth of the sea floor, which means the idealization error is zero. If identification of the bed 
Table 4.5: Sources of error for depth measurements $\underline{d}_{p, s}$ and their variance $\sigma_{p, s}^{(\mathrm{v}) 2}$ for NZO-type vessels. Depth-dependent variances are given, as well as variances for two values of $\underline{d}_{p, s}$. The total propagated error variance $\sigma_{p, s}^{(\mathrm{v}) 2}$ and its $95 \%$ confidence limit $1.96 \sigma_{p, s}^{(\mathrm{v})}$ are also given.

\begin{tabular}{|c|c|c|c|c|}
\hline$i$ & error source $\left[\mathrm{m}^{2}\right]$ & $\overline{\sigma_{i, p, s}^{(\mathrm{v}) 2}}$ & $\begin{array}{c}\sigma_{i, p, s}^{(\mathrm{v}) 2} \\
(15 \mathrm{~m})\end{array}$ & $\begin{array}{c}\sigma_{i, p, s}^{(\mathrm{v}) 2} \\
(25 \mathrm{~m})\end{array}$ \\
\hline 1 & echo sounder & $1 \cdot 10^{-6} d_{p, s}^{2}+6 \cdot 10^{-4}$ & $8 \cdot 10^{-4}$ & $1.2 \cdot 10^{-3}$ \\
\hline 2 & sound velocity profiler & $4 \cdot 10^{-7} d_{p, s}^{2}$ & $1 \cdot 10^{-4}$ & $3 \cdot 10^{-4}$ \\
\hline 3 & degradation of profile & 0 & 0 & 0 \\
\hline 4 & static draught measurement & $6 \cdot 10^{-4}$ & $6 \cdot 10^{-4}$ & $6 \cdot 10^{-4}$ \\
\hline 5 & dynamic draught model & $2 \cdot 10^{-4}$ & $2 \cdot 10^{-4}$ & $2 \cdot 10^{-4}$ \\
\hline 6 & water level reduction & 0.01 & 0.01 & 0.01 \\
\hline 7 & heave sensor & 0 & 0 & 0 \\
\hline 8 & roll sensor & 0 & 0 & 0 \\
\hline 9 & pitch sensor & 0 & 0 & 0 \\
\hline 10 & sea floor idealization & 0 & 0 & 0 \\
\hline & $\sigma_{p, s}^{(\mathrm{v}) 2}\left[\mathrm{~m}^{2}\right]$ & & 0.0117 & 0.0123 \\
\hline & $1.96 \sigma_{p, s}^{(\mathrm{v})}[\mathrm{m}]$ & & 0.21 & 0.22 \\
\hline
\end{tabular}

level would be problematic, e.g. in the presence of a mud layer, the idealization error should be taken into account.

The total error variance follows from the addition of the Kriging variance to the measurement error variance:

$$
\sigma_{p, s}^{(\mathrm{e}) 2}=1.4 \cdot 10^{-6} d_{p, s}^{2}+0.0114+\sigma_{p, s}^{(\mathrm{k}) 2} .
$$

In hydrography, it is common to select the shallowest depth per bin. This operation skews the distribution function of the measurement errors, creating a bias in the shallow direction. Although we are aware of this effect, it is impossible to quantify, unless the original bathymetric data would still have been available. As they are not, we ignore this effect, and do not increase the variance for this. Variances that are modeled too small result in erroneous acceptance of extensions, during the deformation analysis, which is regarded less severe than the opposite erroneous missed dynamics, caused by variances that are modeled too large.

\section{B.3 Variances of the MBES survey}

The MBES-equipped HOV-class survey vessels of the RNLN have replaced the NZO-class vessels. The MBES survey used in this study fully covers the sea floor, allowing us to assign a depth value to each grid node, without applying an interpolation procedure. The error model of the HOV-class vessels is imple- 
mented in the survey software, but is not yet used because the results still show erroneous behaviour. MBES error modeling was first done by Hare [1995].

Instead, the MBES survey uses the S44 order 1 standard for the surveyed depth values [International Hydrographic Organization, 2008a]. MBES uncertainty strongly depends on the transmit and receive angle of the beam, hence swath-width is usually limited by the expected depth uncertainty in relation to the maximum allowed uncertainty [Hare, 1995]. Therefore, we assume that each depth measurement is at least as accurate as specified by S44. Order 1 specifies depth accuracy at the $95 \%$ level as:

$$
1.96 \sigma_{p, s}^{(\mathrm{v})}=\sqrt{a^{2}+\left(b d_{p, s}\right)^{2}}
$$

with depth $d_{p, s}$, and parameters $a$ and $b$, which are $0.5 \mathrm{~m}$ and 0.013 respectively for order 1. For $15 \mathrm{~m}$ and $25 \mathrm{~m}$ depth, the variances for S44, Order 1 are 0.075 $\mathrm{m}^{2}$ and $0.093 \mathrm{~m}^{2}$ respectively, and the corresponding $95 \%$ confidence limits are $0.54 \mathrm{~m}$ and $0.60 \mathrm{~m}$. A comparison with table 4.5 shows that the MBES variance could be almost an order of magnitude more inaccurate than the NZO SBES variance, as the widest MBES beams travel a long distance through the water and are prone to diffraction. In the absence of an interpolation error, and neglecting the positioning error, the MBES error variance is:

$$
\sigma_{p, s}^{(e) 2}=\left(a^{2}+\left(b d_{p, s}\right)^{2}\right) / 1.96^{2} .
$$

For SBES measurements, the interpolation error usually dominates. Therefore, MBES grid nodes are often more accurate than SBES grid nodes, in spite of the larger measurement uncertainty. 


\title{
Chapter 5
}

\section{Spatial variations in sea floor dynamics for the Southern North Sea}

\begin{abstract}
The optimization of the resurvey policy of the Netherlands Hydrographic Service requires insight into sea floor dynamics in the Southern North Sea. To study the spatial variations in sea floor dynamics, the bathymetric archives of the Netherlands Hydrographic Service are analyzed using deformation analysis. We focus on several sand wave areas and a single flat area. In some of those areas, dredging takes place, to guarantee a minimum depth. The results indicate sand wave migration in areas close to the coast, and bed level changes of the order of decimeters over the past two decades. The dominant wavelength of the sand waves varies. We compare our results to earlier studies of the same sand wave areas, in which we find similar migration rates, but different wavelengths.
\end{abstract}

\section{$5.1 \quad$ Introduction}

The sea floor of the Southern North Sea on the Belgian Continental Shelf and the Southern part of the Netherlands Continental Shelf is covered with intricate rhythmic bed form patterns. Bed form patterns, induced by the interaction of tidal flows and sandy sediments and with a wavelength of about 100 to $1000 \mathrm{~m}$, are called tidal sand waves. The presence and dynamics of tidal sand waves can have a significant impact on the navigation depth, with migration rates that depend on the tidal flow conditions. Idealized process-based models for tidal sand wave behaviour make an important contribution to the understanding of these dynamics [Besio et al., 2008; Németh et al., 2007].

In the Southern North Sea, the influence of sand wave dynamics on depth has recently been studied by e.g. Van Dijk et al. [2008], Knaapen [2005] and Wüst [2005], for areas that are intensively used for navigation. An overview of the sand wave characteristics, as found by Van Dijk et al. [2008], Knaapen [2005] and Knaapen et al. [2001], is given in Table 5.1. In these studies, advantage is taken of the modern surveys using multi-beam echo sounders (MBES), which provide high-resolution depth data. The high spatial resolution requires highly automated procedures. In contrast, older analyses that use single-beam echo sounders (SBES) choose an interpretation based on visual evaluation of depth values, see e.g. Wright [1992]; Kember [1984]; Langhorne [1982a]; Ter- 
Table 5.1: Dominant wavelengths $L^{(\mathrm{d})}$ and migration rates $\dot{\xi}^{\prime}$ on the Belgian and Netherlands Continental Shelf for the regions Selected Track and Noordhinder, as found in literature. The wavelengths found by Knaapen [2005] are rounded to the nearest $10 \mathrm{~m}$. The areas are shown in Figure 5.2.

\begin{tabular}{|c|c|c|c|}
\hline location & $L^{(\mathrm{d})}[\mathrm{m}]$ & $\dot{\xi}^{\prime}[\mathrm{m} / \mathrm{yr}]$ & reference \\
\hline Noordhinder region & 550 & - & Knaapen et al. [2001] \\
\hline Twin area & 160 & -0.6 & Knaapen [2005] \\
\hline \multicolumn{4}{|l|}{ Noordhinder Junction: } \\
\hline subarea 1 & 250 & -0.2 & Knaapen [2005] \\
\hline subarea 2 & 220 & -0.2 & Knaapen [2005] \\
\hline Short Stay anchorage area & 240 & 0.1 & Knaapen [2005] \\
\hline \multicolumn{4}{|l|}{ Eurogeul Approach: } \\
\hline subarea 1 & 230 & 0.2 & Knaapen [2005] \\
\hline subarea 2 & 240 & 0.3 & Knaapen [2005] \\
\hline Eurogeul Approach & 160 & 0.4 & Van Dijk et al. [2008] \\
\hline
\end{tabular}

windt [1971]. Most of the studies on sand wave dynamics in the North Sea indicate sand wave migration. Such information helps the resurvey planning of bathymetric surveys by the Netherlands Hydrographic Service, used for nautical charting. This is crucial for the safe navigation through one of the busiest shallow seas in the world.

The study of spatial variations in sea floor dynamics from surveys, at the scale of the full Belgian and Netherlands Continental Shelf (BNLCS), is complicated by the absence of series of surveys for the largest part of the BNLCS. However, series of surveys have become available for several areas over the past decade. In this study, we present the results of the deformation analysis (Chapter 3) performed on several areas on the BNLCS, and we compare them with the results from existing methods.

Our study answers a methodical question, on the performance of deformation analysis for areas with a rather complicated morphology, and a morphological question, on the role of sand wave patterns in sea floor dynamics. The answers to the methodical question help us to explain differences with the existing results. To answer the morphological question, we try to find correlations between sand wave migration and other morphological parameters.

In order to apply the method of deformation analysis, a two-dimensional approach is chosen, for which areas are split up into smaller subareas of equal size. Such an approach results in a limited number of morphological parameter estimates, allowing for the most insightful description of a full area. For each subarea, we define a grid. Results of one-dimensional analyses of each individual grid line in the direction perpendicular to the sand wave crests, and zero-dimensional analyses per individual grid node potentially differ from these 
two-dimensional results. If this is the case, these additional results are given as well.

The presented areas have been selected because of the availability of at least three surveys. The spatial extent of a subarea is subject to the following constraints. It must be small enough to assume uniform morphology, and large enough to cover at least a full wavelength of a sand wave. The grid spacing depends on the presence of sand waves, which limits the maximum extent for computational reasons. Details on deformation analysis and its settings are given in Section 5.2.

We consider four regions, shown in Figure 5.1 and Table 5.2:

1. the Selected Track region for vessels with a deep draught, between the English Channel and the Noordhinder Junction (Section 5.3);

2. the Noordhinder region, West of the Port of Rotterdam (Section 5.4);

3. the region West of the Port of IJmuiden (Section 5.5);

4. the region North of Terschelling (Section 5.6);

A fifth region West of Rotterdam was analyzed in Chapter 4, also using deformation analysis. Both the regions Noordhinder and West of IJmuiden have a guaranteed depth, which requires human intervention in the natural morphological system by repetitive dredging. All regions show sand wave patterns, except for the region North of Terschelling. The areas of each region are listed in Table 5.2. The areas in the regions Selected Track and Noordhinder are also shown in Figure 5.2. The results are used to discuss the morphological question and the methodical question in Section 5.7. Conclusions on the variation between the detected dynamics of those areas are drawn in Section 5.8.

\subsection{Deformation analysis}

\subsubsection{Overview}

\section{Input and Output}

Deformation analysis is an application of statistical testing theory [e.g. Koch, 1999] to analyze series of bathymetric surveys $s=1, \cdots, S$. In order to apply deformation analysis to the sea floor, we use depth values at the nodes $p=1, \cdots, P$ of a grid, the uncertainty of each depth value and the time of each survey. The size of the grid is limited to about $20 \times 20$ nodes, for computational reasons. The uncertainty is described by an error model, giving variances and covariance functions for the used surveys, see Chapter 4 for the Royal Netherlands Navy (RNLN) SBES surveys. Further, it is assumed that the performance of the Rijkswaterstaat (RWS) and RNLN MBES survey suites is according to order 1 of the S44 standard [International Hydrographic Organization, 2008a].

The procedure allows us to select $N$ significant morphological and morphodynamic parameters for an area, based on $M$ depth values, where $M=P S$. 


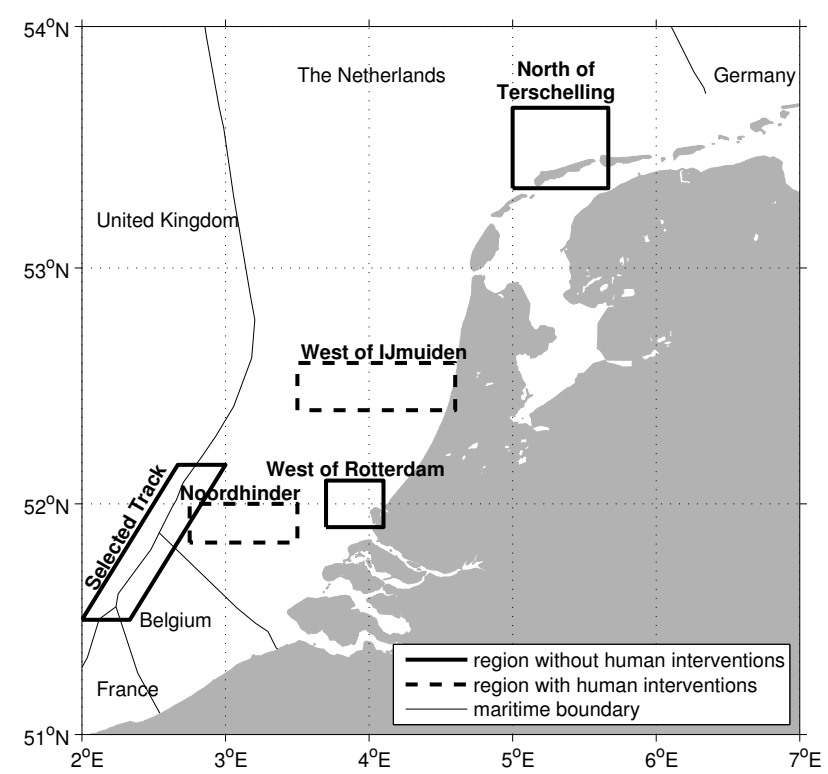

Figure 5.1: Overview of analyzed regions in the Southern North Sea, on the Belgian and Netherlands Continental Shelf. The region West of Rotterdam is analyzed in Chapter 4.

Subsequently, deformation analysis is able to provide estimates for these parameters, including confidence intervals. This results in a representation of the measured sea floor that consists of the characterization by a limited number of parameters, and the dispersion of the residual variations from the characterization, which is described by the variances and covariances of the parameters. The relations between the depth values and the estimated parameters are linear or linearized. This allows us to follow a Best Linear Unbiased Estimation (BLUE) for the estimation of the parameters of the characterization, and for the estimation of their dispersion. More details are given in Chapter 3.

\section{Morphological characterization}

Let us assume the input is available in a three-dimensional coordinate frame $(x, y, z)$, in which the three directions are perpendicular to each other, and depth $d$ is given as $z=-d$ at horizontal locations $(x, y)$. Deformation analysis subsequently tests extensions of the set of morphological parameters, starting with the simplest sea floor characterization possible, which is the single parameter bed level $d^{(\mathrm{b})}$, assumed constant in space and time. Then, spatial or temporal extensions to this set are made. Such an extension is accepted if its so-called test quotient $\underline{q}$ is the largest over all available extensions, and if this test quo- 


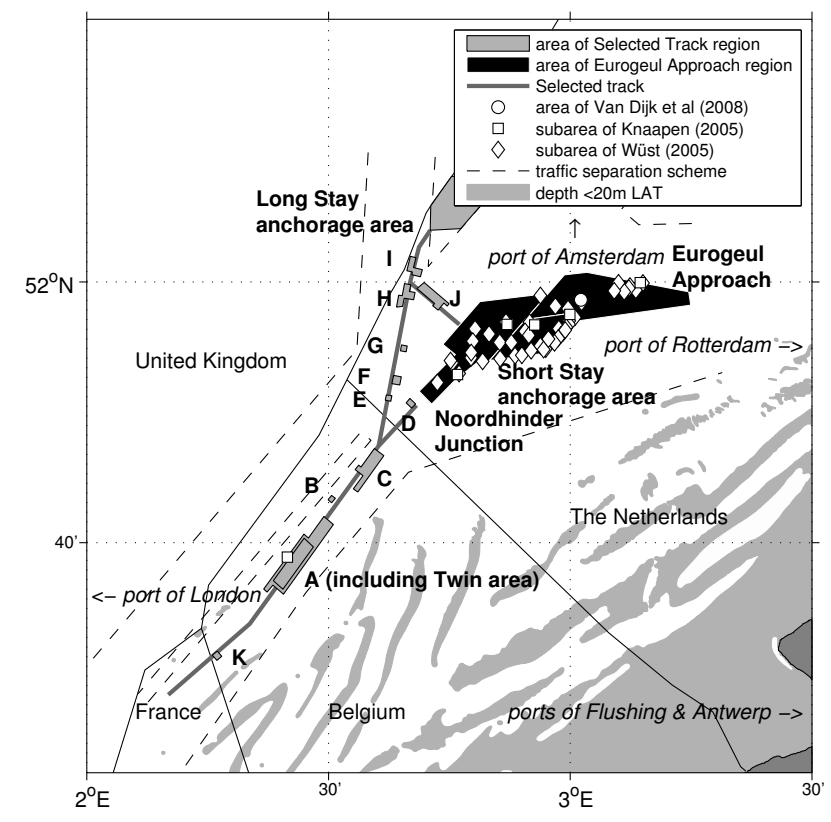

Figure 5.2: Overview of analyzed areas on the Belgian and Netherlands Continental Shelf in the Selected Track region without guaranteed depth, and the Noordhinder region with guaranteed depth.

tient is larger than one. The characterization is extended until the remaining test quotients are all smaller than one.

Spatial extensions (step 1) to the initial parameter bed level $d^{(\mathrm{b})}$ are two bed slope parameters $\psi^{(\mathrm{x})}$ and $\psi^{(\mathrm{y})}$ in both the horizontal directions, and two sand wave parameters: amplitude $A$ and crest position $\xi$. Sand wave patterns are assumed to be regular, such that a horizontal direction of the pattern variation can be identified as the $x$-direction of the grid, perpendicular to the direction of along-crest uniformity, which is the $y$-direction of the grid. We usually work with the relative sand wave parameters, with respect to their values $A_{\text {ref }}$ and $\xi_{\text {ref }}$ for a reference survey: amplitude factor $A^{\prime}=A / A_{\text {ref }}$ and migration $\xi^{\prime}=\xi-\xi_{\text {ref }}$. To determine the dominant wavelength $L^{(\mathrm{d})}$ of the sand wave extension, the test quotient becomes a function of wavelength: $\underline{q}=\underline{q}(L)$. Consequently, a wavelength search interval $\left[L_{\min }, L_{\max }\right]$ needs to be defined. Subsequently, the best fitting wavelength is obtained by maximizing $q(L)$.

The dynamic extensions (step 2) define a linear parameter trend in all surveys (denoted e.g. $\dot{d}^{(\mathrm{b})}$ for bed level), and outlying parameters for survey $s$, for each $s=1, \cdots, S$ (denoted e.g. $\Delta d_{s}^{(\mathrm{b})}$ for bed level). Because the two parameters $\psi^{(\mathrm{x})}$ and $\psi^{(\mathrm{y})}$, and the two parameters $A$ and $\xi$ are mutually correlated, an extension contains the two bed slope parameters, or the two sand wave param- 
Table 5.2: Areas per region and number of subareas per area, including the area codes used in the Table 5.3.

\begin{tabular}{llr}
\hline \hline region & areas (area code) & \# subareas \\
\hline Selected Track & Critical area A, & \\
& $\quad$ including Twin (ST1); & 25 \\
& Critical areas B, & 20 \\
& $\quad$ E, F, G, H, I, J (ST2); & 11 \\
& Critical areas C, D, K (ST3); & 32 \\
& Long Stay anchorage (ST4) & 72 \\
Noordhinder & Noordhinder Junction (NH1); & 24 \\
& Short Stay anchorage (NH2); & 92 \\
& Eurogeul Approach (NH3) & 18 \\
West of Rotterdam & Maas West anchorage (WR1) & 108 \\
West of IJmuiden & IJgeul Approach (WIJ1); & 12 \\
& IJgeul Approach anchorage (WIJ2) & 14 \\
\hline \multirow{2}{*}{ North of Terschelling } & shallow part of TE-TSS (NT1) & \\
\hline \hline
\end{tabular}

eters. First, these two trend extensions are tested. Depending on the values of the test quotients, the trend extensions are added to the characterization. After that the $2 S$ outlier extensions are tested, to account for nonlinear behaviour or systematic errors in a survey.

The levels of significance $\alpha$ specify the probability that an extension to a representation is incorrectly selected. We use the values for $\alpha$ that were set in Chapter 4 , which are 5\%,7\% and $5 \%$ for the spatial extensions, the trend extensions and the outlier extensions, respectively. Deformation analysis is possible using three different numbers of dimensions: a zero-dimensional analysis per grid node, a one-dimensional analysis per grid line in the direction of highest variability, and a two-dimensional analysis of a full grid. The specified levels of significance are adopted for all three these numbers of dimensions.

\section{Morphological residuals}

The iterative acceptance of extensions continues until all remaining extensions have a test quotient smaller than one. If the characterization fits the series of surveys well enough, from a statistical point of view, the final parameter values are calculated, as well as the associated uncertainties. The overall test is used for this decision, with its overall test quotient $\underline{q}^{(\mathrm{o})}$ smaller than one to pass the test.

However, if the differences between the characterization and the measured sea floor are larger than can be explained by the uncertainty of the depth measurements, the characterization is insufficient. The differences are called the residuals, which contain measurement errors and residual variations, like asymmetries and bifurcations in the sand wave pattern, and small-scale rhythmic 
features like mega-ripples. Instead of defining additional parameters, which would complicate the characterization, we assume that the residual variation is a random function, and we include additional morphological variance and covariance components in the uncertainty, using Least Squares Variant Component Estimation (LSVCE) [Teunissen and Amiri-Simkooei, 2008].

The LSVCE-procedure is followed for both the steps explained above. The variances and covariances, describing the dispersion, that are calculated in step 1 are used in step 2. An increased dispersion during step 1 therefore has consequences in step 2. In the first place, it is less likely that an extension will be accepted in that step. Also, it means that the resulting dynamic parameters will be estimated less accurately. At the end of step 2, the second LSVCE procedure only influences the uncertainty of the final parameter estimates.

\subsubsection{Wavelength}

The areas analyzed using deformation analysis are listed in Table 5.3. To determine the dominant wavelength $L^{(\mathrm{d})}$ of the sand waves, it is necessary to define search intervals $\left[L_{\min }, L_{\max }\right]$. The minimum wavelengths $L_{\min }$ are chosen by visual inspection of the crest distances, and we define $L_{\max }=2 L_{\min }$, wherever possible. This excludes additional maxima in the test quotient function at multiples of the dominant wavelength. The results in literature (Table 5.1), which are not consistent with each other, are contained in our search intervals, except for the wavelengths of Van Dijk et al. [2008]. The differences are due to the high complexity of the sand wave pattern in the Noordhinder region.

Table 5.1 shows that wavelengths of the order of both $250 \mathrm{~m}$ and $550 \mathrm{~m}$ were found for the three connected areas Noordhinder Junction, Approach Eurogeul and Short Stay anchorage. The simultaneous presence of these two patterns forces us to choose a larger wavelength search interval. This is also necessary for the IJgeul Approach area.

For the Critical areas, either the smaller or the larger wavelength seems to dominate an area. The Southern areas usually show a small dominant wavelength, and the Northern areas a large dominant wavelength. For Critical area A, which includes the Twin area, an even smaller wavelength is present.

By definition, the $x$-direction of the grids coincides with the direction of highest variability $\underline{\theta}^{(\mathrm{x})}$ after correcting for the slope of a subarea, according to the DIGIPOL-method [Van Munster et al., 1995; RIKZ, 1997; De Koning, 2007]. This direction is obtained by maximising the DIGIPOL variability function $\underline{v}(\theta)$, for which the quotient $\max (\underline{v}) / \min (\underline{v})$ quantifies the strength of the direction. The grid spacing $h^{(\mathrm{g})}$ is set according to the last column in Table 5.3. In subareas where a sand wave may be present, the grid spacing must be smaller than half the wavelength, to prevent aliasing effects. For these areas, a grid spacing of $50 \mathrm{~m}$ is chosen. The shallowest part of the Traffic Separation System North of Terschelling (TE-TSS) does not show any rhythmic patterns, thereby allowing for larger grid spacings, and consequently larger subareas are analyzed at once. 
Table 5.3: Search intervals $\left[L_{\min }, L_{\max }\right]$ for wavelength $L^{(\mathrm{d})}$, and grid spacing $h^{(\mathrm{g})}$ used for the analyzed areas. The area codes have been introduced in Table 5.2.

\begin{tabular}{lccc}
\hline \hline area code & $L_{\min }[\mathrm{m}]$ & $L_{\max }[\mathrm{m}]$ & $h^{(\mathrm{g})}[\mathrm{m}]$ \\
\hline ST1 & 150 & 300 & 50 \\
ST2 & 450 & 900 & 50 \\
ST3 & 200 & 400 & 50 \\
ST4 & 200 & 900 & 50 \\
NH1-4 & 200 & 900 & 50 \\
WR1 & 200 & 400 & 50 \\
WIJ1-2 & 200 & 900 & 50 \\
NT1 & - & - & 125 \\
\hline \hline
\end{tabular}

\subsection{The Selected Track region: little dynamics}

Results for all Critical areas were presented before by Dorst et al. [2007]. The results presented here differ, because of changes made to the application of deformation analysis, and because of the usage of additional surveys. The application of deformation analysis now includes an LSVCE procedure, which prevents the misinterpretation of small scale dynamics as dynamics at the scale of a subarea.

\subsubsection{Spatial characterization}

The available number of surveys for the Critical areas and the Long Stay anchorage area are shown in Figure 5.3, for each subarea. All surveys are listed in Appendix 5.A. There are many surveys available for the Twin area, which we split up, for computational reasons, into three analysis periods: 1991 to 1999, 2000 to 2002, and 2003 to 2006. This abundance of data is caused by a shared surveying effort of Rijkswaterstaat (RWS) and the Royal Netherlands Navy (RNLN). The RNLN surveys the Twin area as part of Critical area A. Figure 5.3 also shows the characterization of the sea floor by step 1 of the deformation analysis procedure. Each subarea is characterized as one of the following types: a horizontal plane, a sloping plane, a horizontal plane with a sand wave pattern, or a sloping plane with a sand wave pattern. It turns out that most of the subareas are characterized by the deformation analysis as both showing slopes and a sand wave. For those subareas in which no sand wave pattern is detected, the pattern is in fact too irregular: it differs too much from a sine shaped wave in a single direction.

\section{Twin area}

Most subareas of the Twin area show the same characterization for all three analysis periods. An exception is the subarea indicated by the arrow in Figures 5.3c-e, which is characterized as horizontal first (1991-1999), then as sloping with a sand wave pattern (2000-2002), and finally as sloping without a sand wave 

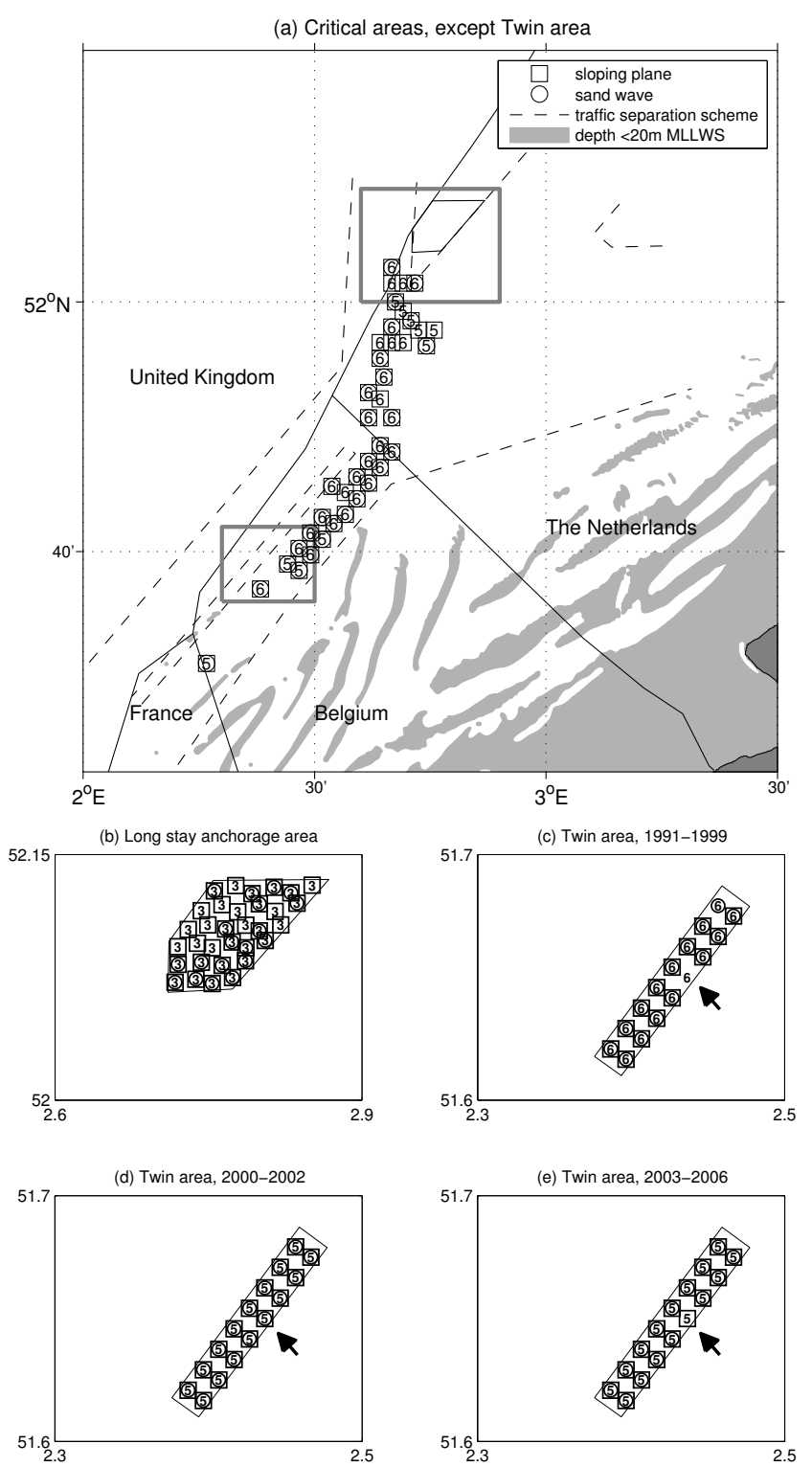

Figure 5.3: The analyzed number of surveys and the characterizations for (a) the Critical areas except for the Twin area, (b) the Long Stay anchorage area, and (c)-(e) the Twin area. The subarea indicated by the arrow in the Figures (c)-(e) is shown in detail in Figure 5.4. Most of the subareas are characterized by both a spatial extension for slope, and a spatial extension for a sand wave pattern, visible as the presence of both a circle and a square around the number. 
pattern (2003-2006). This exceptional subarea is shown in Figure 5.4a, which shows bifurcating crests. The bifurcation increases the dominant wavelength to the maximum value of the search interval, as visible in Figure 5.4b. The test quotients $\underline{q}(L)$, as a function of wavelength $L$, for the 2000-2002 period has its maximum just within the search interval, at $L^{(\mathrm{d})}=296 \mathrm{~m}$. For the other two analysis periods, the test quotient has its maximum value outside the search interval; in that case no sand wave pattern is accepted.

The test quotients $q(L)$ are larger for analysis periods that have relatively more MBES surveys. The three analysis periods contain zero, two, and four MBES surveys respectively. MBES surveys have, on average, more accurate gridded depth values. Therefore, it is easier to detect slopes and sand wave patterns. This effect is visible for the test quotients plotted in Figure 5.4b. The test quotients for the slopes are $0.23,1.23$, and 3.33 for the three periods, respectively.

\section{Regularity}

The orientations of the sand wave pattern , calculated as the DIGIPOL-direction of highest variability $\underline{\theta}^{(\mathrm{x})}$, are shown in Figure 5.5. The directions are based on the May 2000 SBES survey of the Selected Track. Applying the DIGIPOLprocedure to a data set for which the data resolution strongly depends on direction, like SBES surveys, results in a bias toward the direction of highest resolution. The DIGIPOL-procedure is computationally too expensive to be applied to a MBES survey. Fortunately, the survey ship commander usually plans the SBES survey tracks roughly in the direction of the sand wave pattern, to prevent the missing of crests in the data.

The May 2000 survey was sailed in the directions $36^{\circ}$ and $216^{\circ}$. Figure 5.5 shows that the resulting DIGIPOL directions show differences of up to $30^{\circ}$ with respect to the track direction. Figure 5.5 also shows that the Southern subareas have a more clearly defined direction of highest variability than the Northern subareas, as the DIGIPOL-quotients are larger in the South. This indicates that the sand waves are less regular in the Northern subareas.

The decrease in regularity toward the North is also seen in Figure 5.6, as indicated by the cross symbols for sand wave absence. Other potential causes for the rejection of the sand wave extension are the limited pattern amplitude, or the boundaries of the wavelength search interval (as we saw in Figure 5.4). As we have defined the wavelength search interval differently for Critical area A, the other Southern areas, and the Northern areas (see Table 5.3), the wavelengths clearly differ. The largest amplitudes of the areas with a sand wave characterization are found for the Northern Critical areas, where we also found larger wavelengths. The patterns for the Long Stay anchorage area are more complicated, which reduces the detected sand wave amplitude. 

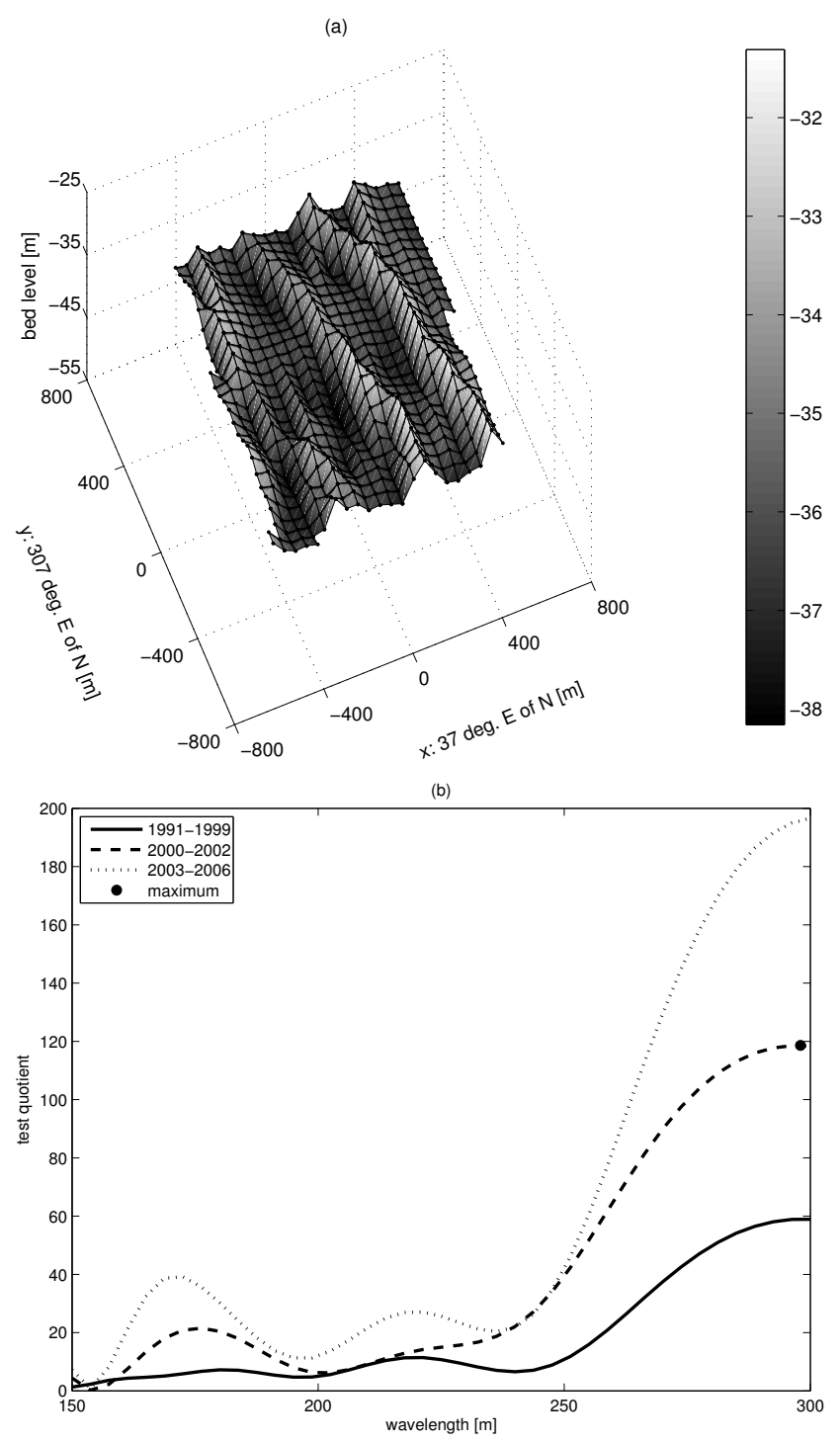

Figure 5.4: Characterization of the exceptional subarea of the Twin area: (a) gridded depth values, showing a bifurcation; (b) test quotients $\underline{q}(L)$, for the three analysis periods. The maximum test quotient is found for the wavelength at the maximum of the search interval, because of the bifurcation. 

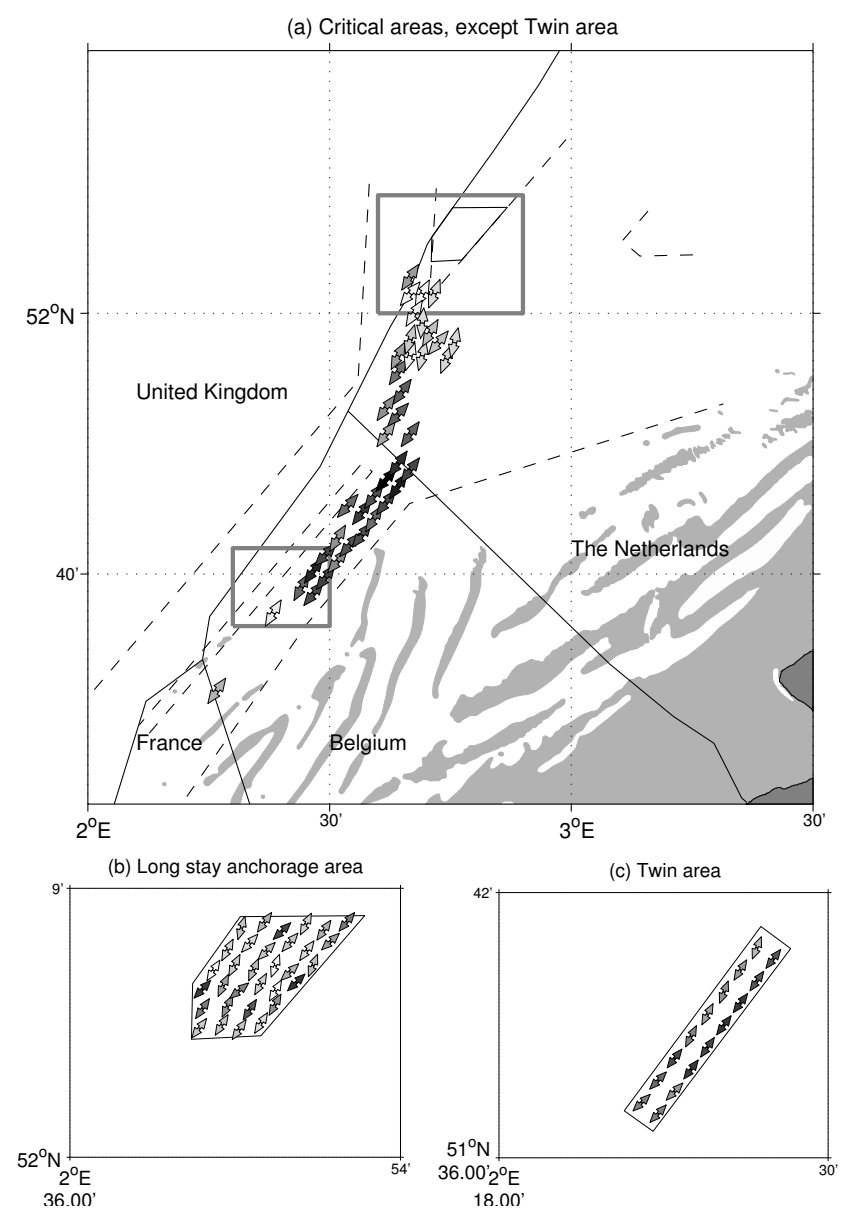

Figure 5.5: DIGIPOL directions $\underline{\theta}^{(\mathrm{x})}$ and quotients $\max (\underline{v}) / \min (\underline{v})(1.4$ to 3.7 , darker is larger) for (a) the Critical areas except for the Twin area, (b) the Long Stay anchorage area, and (c) the Twin area.

\subsubsection{Temporal characterization}

The two-dimensional deformation analyses in the Twin area do not reveal any migration of the sand wave patterns. However, Knaapen [2005] found small migration rates using an alternative method, see Table 5.1. To estimate migration rates, Knaapen [2005] removed systematic positioning errors from the data, and identified crest and trough positions. According to our results using deformation analysis, these rather small migration rates are not present, and therefore qualified as artifacts, caused by remaining horizontal measurement inaccuracies, identification inaccuracies of the crests and troughs, or local shifts of a single crest or trough. If these migration rates are real, our representation of a sand 

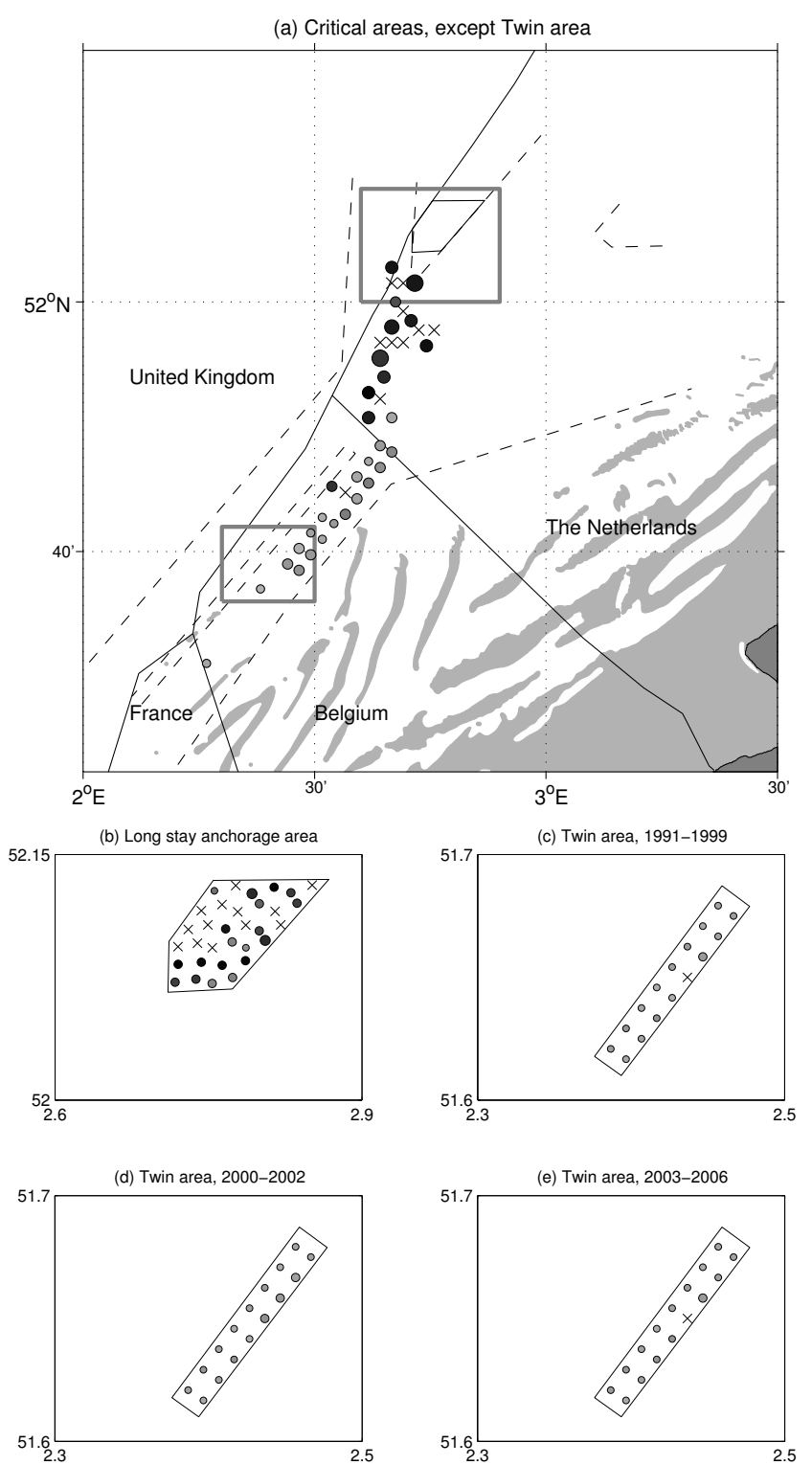

Figure 5.6: Dominant wavelengths $\underline{L}^{(\mathrm{d})}$ (165 to $886 \mathrm{~m}$, shown by shade of the circles, darker is larger wavelength) and amplitudes $\underline{A}$ ( 0.1 to $4.2 \mathrm{~m}$, shown by size of the circles, larger is a larger wavelength) for (a) the Critical areas except for the Twin area, (b) the Long Stay anchorage area, and (c)-(e) the Twin area. A cross symbol denotes a subarea where the sand wave pattern is too irregular, the amplitude is too small to detect the pattern, or the dominant wavelength is outside the specified search interval. 
wave pattern by a sine function does probably not detect these values in such a complicated sand wave field. Our results for bed level dynamics are given in Figure 5.7. Downward trends were found for the first analysis period of the four most Southwestern subareas of the Twin area, see Figure 5.7c.

\section{Twin area}

There could also be small trends in the parameters of the Twin area that are not detectable in the separate analyses of any of the three analysis periods, but that are detectable over the full time span of available surveys. However, analyzing this full time span is computationally far too expensive. To circumvent this problem, we follow two approaches. The first approach is to take every third available survey only. It turns out that this does not result in the detection of dynamics for any subarea that was classified as static for the three original analysis periods. An outlying bed level $d^{(\mathrm{b})}$ was found for the first survey, for two subareas out of the four subareas that showed a downward trend for the first analysis period. These are the Northern and the Western subarea. This confirms the detection of the downward trend for the first analysis period for these subareas: the initial downward trend did not continue long enough to be detected by the trend test of the full time span, but the first survey was too shallow to be considered part of a static bed level.

The second approach is to apply deformation analysis to the three resulting sets of parameters only, rather than the bathymetric data set. A static representation for a parameter over the full time span of the surveys is defined as a null hypothesis, and a representation that includes a linear trend for that parameter as an extension, or alternative hypothesis. The data are the parameter values for the three analysis periods, including their variances. In the case of a trend in an analysis period, the parameter used is the reference parameter $u_{\text {ref }}$.

This approach fails for the sand wave parameters, as the wavelength is not assumed constant over the separate analysis periods. For the bed level parameter $d^{(\mathrm{b})}$, this results in the detection of long term downward trends for the four subareas for which a downward trend was already found during the first analysis period. Also, an additional long term trend was found for one more subarea. These subareas are marked by a plus sign in Figure 5.7. The detected long term trends have a maximum of $0.03 \mathrm{~m} / \mathrm{yr}$, with a $95 \%$ confidence interval of 0.01 $\mathrm{m} / \mathrm{yr}$.

The differences between the original approach that uses three analysis periods for the Twin area, and the two alternative approaches described above are small. We conclude that the choices for the specific analysis period for deformation analysis does not make a big difference, and use the results of the original approach with three analysis periods.

\section{Grid line analyses}

The one-dimensional results per grid line show some exceptional dynamics that are hardly larger than their uncertainty at a 95\% confidence interval, see Ta- 

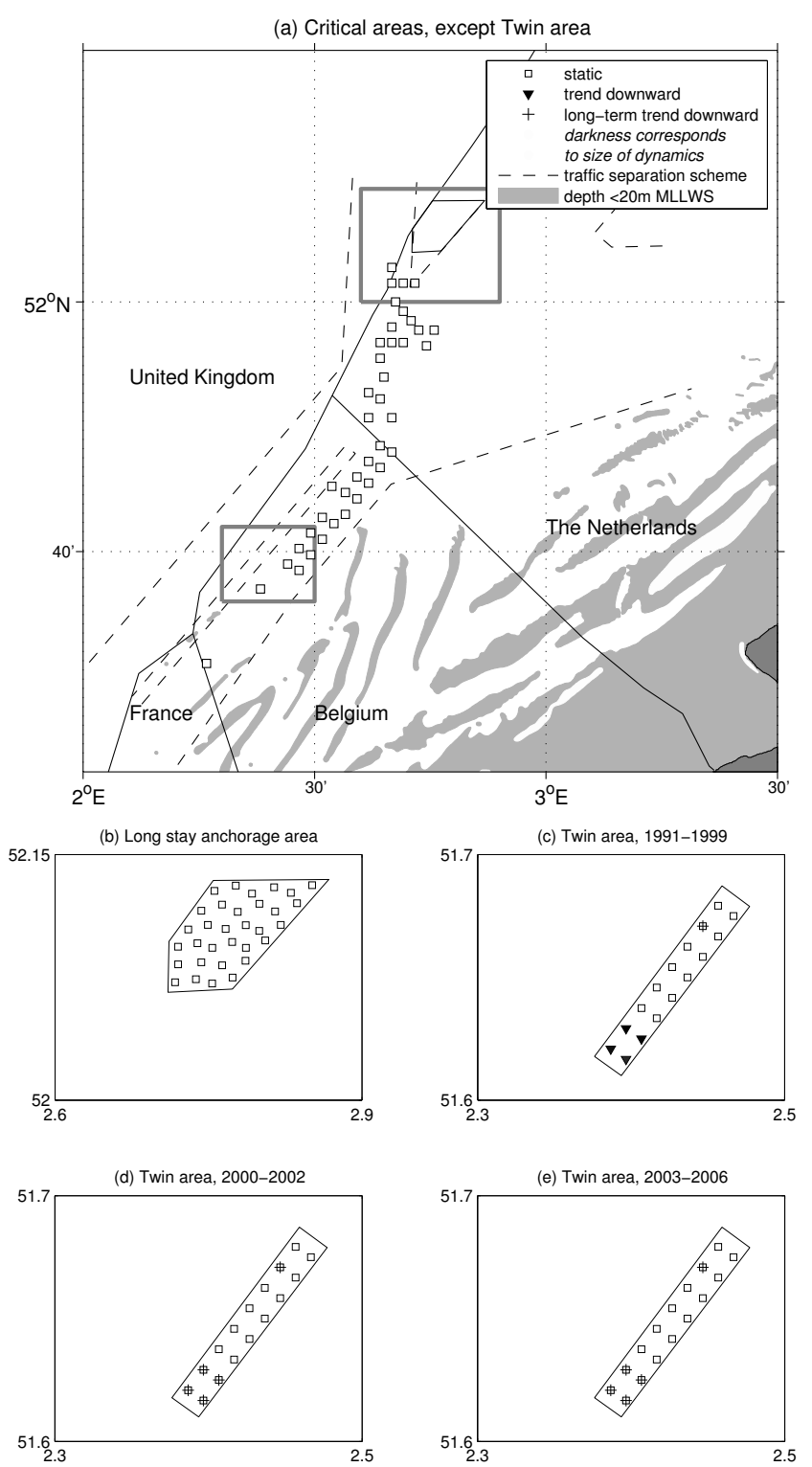

Figure 5.7: Dynamics of Bed level $\underline{d}^{(\mathrm{b})}$ for (a) the Critical areas except for the Twin area, (b) the Long Stay anchorage area, and (c)-(e) the Twin area. (The values of the detected downward trends for the analysis period 1991-1999 of the Twin area are between 0.04 and $0.05 \mathrm{~m} / \mathrm{yr}$, with an uncertainty at a $95 \%$ confidence interval of 0.01 to $0.03 \mathrm{~m} / \mathrm{yr}$. The values of the detected long-term downward trends of the Twin area are between 0.01 and $0.03 \mathrm{~m} / \mathrm{yr}$, with an uncertainty at a $95 \%$ confidence interval of $0.01 \mathrm{~m} / \mathrm{yr}$.) 
Table 5.4: Grid line dynamics for the Selected Track region. For this region, 88 analyses were done with approximately 20 grid lines per subarea. All results were static, except for the ones listed here. The uncertainty is expressed at a $95 \%$ confidence interval.

\begin{tabular}{llcc}
\hline \hline type of dynamics & location, & \multicolumn{2}{c}{ maximum } \\
(number of grid lines) & analysis period & estimate & uncertainty \\
\hline linear downward trend (9) & Twin, 1991-1999 & $0.005 \mathrm{~m} / \mathrm{yr}$ & $0.005 \mathrm{~m} / \mathrm{yr}$ \\
shallower outlier (1) & Twin, 1991-1999 & $0.4 \mathrm{~m}$ & $0.4 \mathrm{~m}$ \\
shallower outlier (1) & Critical area J & $0.6 \mathrm{~m}$ & $0.3 \mathrm{~m}$ \\
smaller amplitude outlier (1) & Twin, 1991-1999 & $-40 \%$ & $50 \%$ \\
positive position outlier (1) & Twin, 1991-1999 & $18 \mathrm{~m}$ & $19 \mathrm{~m}$ \\
\hline \hline
\end{tabular}

ble 5.4 for the details. These dynamics may be artifacts of the method. For instance, the crest position outlier for the sand wave may be caused by the less accurate positioning equipment used during the May 1995 survey. Only the nine detected downward trends are realistic, because they confirm the twodimensional grid analyses of the Southwestern subareas of the Twin area between 1991 and 1999, shown in Figure 5.7.

For most of the subareas, the static results of the two-dimensional analyses are confirmed by static results of the one-dimensional analyses. We conclude that the choices for the dimensions of deformation analysis do not make a significant difference.

\subsection{The Noordhinder region: comparison with other methods}

The areas studied in this Section are the Eurogeul Approach channel, the Short Stay anchorage area, and the Noordhinder Junction (Figure 5.2), based on the surveys listed in Appendix 5.A. These three areas are among the most critical on the BNLCS, because of the combination of a high shipping intensity, small depth values, and a dynamic sea floor. The areas are surveyed by Rijkswaterstaat several times a year, and the depth is maintained by dredging, which took place in 1991, 1996, 2002, and 2004. During those dredging operations, the sediment was removed from the area, except for 2002, when the sediment taken from the crests was placed in the troughs [Stolk, personal communication]. The sea floor dynamics of these areas have been studied before, using alternative methods [Van Dijk et al., 2008; Knaapen, 2005; Wüst, 2005]. We present our results in Section 5.4.1 and 5.4.2, and compare our results from the deformation analyses with the results in literature in Section 5.4.3. 


\subsubsection{Spatial characterization}

The sand wave patterns in the three areas are complicated, because of the simultaneous presence of several wavelengths, first described by Knaapen et al. [2001]. An impression of the pattern is given by Figure $5.8 \mathrm{~b}$ for one of the subareas in the Southwest of the Short Stay anchorage area. See Knaapen et al. [2001] for a high-resolution view of the pattern. However, the directions of highest variability are rather uniform over the three areas, as shown by Figure 5.8a. The superposition of the sand wave patterns makes it difficult to find a dominant wavelength per subarea: a pattern with a wavelength of about $160 \mathrm{~m}$ [Van Dijk et al., 2008], about $250 \mathrm{~m}$ [Knaapen, 2005], and about $550 \mathrm{~m}$ [Knaapen et al., 2001] are reported, see Table 5.1. We only observe the two larger wavelengths, and consequently set the boundaries of the search interval for deformation analysis at $L_{\min }=200 \mathrm{~m}$ and $L_{\max }=900 \mathrm{~m}$. The resulting dominant wavelengths are distributed over the full search interval, while their spatial distribution seems random.

Figure 5.9 shows histograms for the individual areas, and for the three areas as a whole. Since the histogram of all areas is based on more data, it is possible to have bins at a higher resolution. Peaks are observed around 400 and $700 \mathrm{~m}$, which differs from the values in literature (Table 5.1). Also compare the SBES wavelengths of the Noordhinder Junction with the MBES wavelengths. The distribution of the MBES wavelengths is more peaked, around the two wavelength maxima. For the other two areas, mainly MBES surveys are used; they show clearer maxima than the Noordhinder junction SBES wavelengths. We explain this by the absence of an interpolation procedure for MBES surveys. The interpolation must be done in the across-track direction, which usually coincides with the direction of the crests. Therefore, interpolation creates additional spurious structures that are elongated in the crest direction, thus disturbing the original pattern.

\subsubsection{Temporal characterization}

\section{Grid analyses}

For the Short Stay anchorage area and the Eurogeul Approach area, dynamics were found by neither the two-dimensional deformation analyses, nor the one-dimensional deformation analyses. For the Noordhinder Junction, the twodimensional analyses of SBES surveys show a downward trend in bed level $d^{(\mathrm{b})}$ for almost every subarea, in some cases accompanied by outliers. The analyses of the MBES surveys do not show such a trend anymore, only some outliers. The results are presented in Figure 5.10. The four SBES surveys were carried out between 1988 and 1995, and the six MBES surveys between 2001 and 2006. Such a sudden change between these two analysis periods is most likely due to human activities. A potential explanation is the change in dredging strategy that was made around the year 2000: the sediment dredged from the sand wave crests was not removed anymore, but stored in the troughs. This means that 


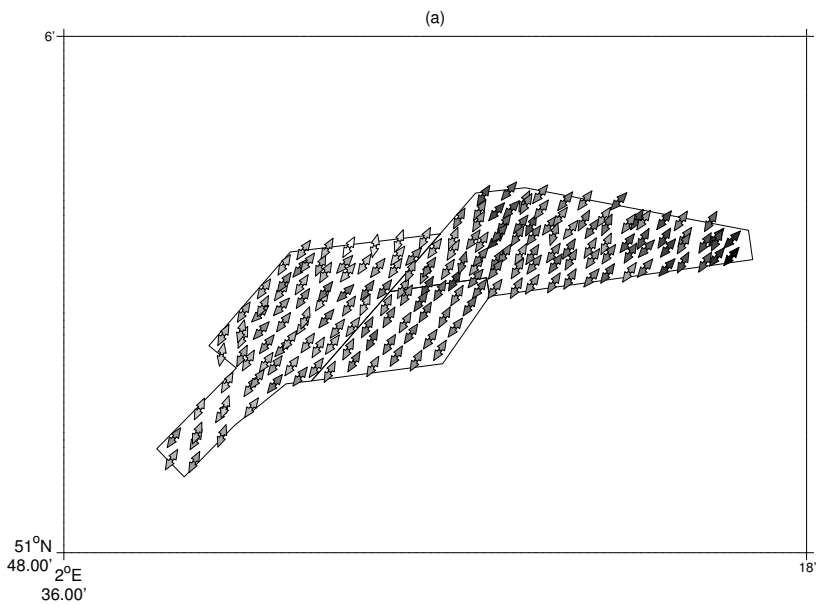

(b)

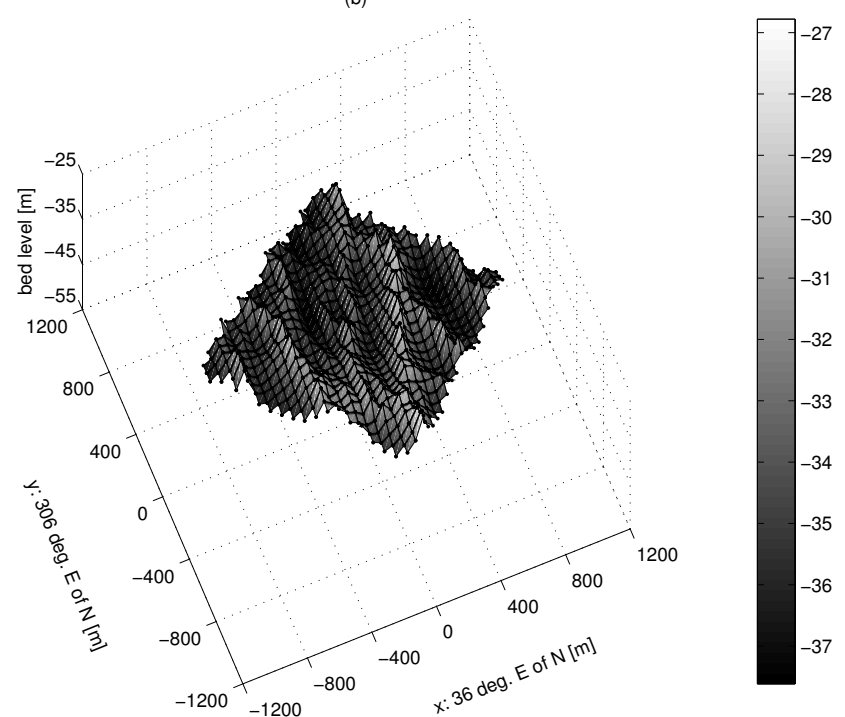

Figure 5.8: Direction of the sand wave pattern for the Noordhinder Junction, the Eurogeul Approach area, and the Short Stay anchorage area: (a) DIGIPOL directions $\underline{\theta}^{(\mathrm{x})}$ and quotients $\max (\underline{v}) / \min (\underline{v})$ (quotients 1.5 to 4.4 , darker is larger); (b) an example of the pattern: the gridded depth values of a subarea in the Southwest of the Short Stay anchorage area, based on the June 2005 MBES survey. 

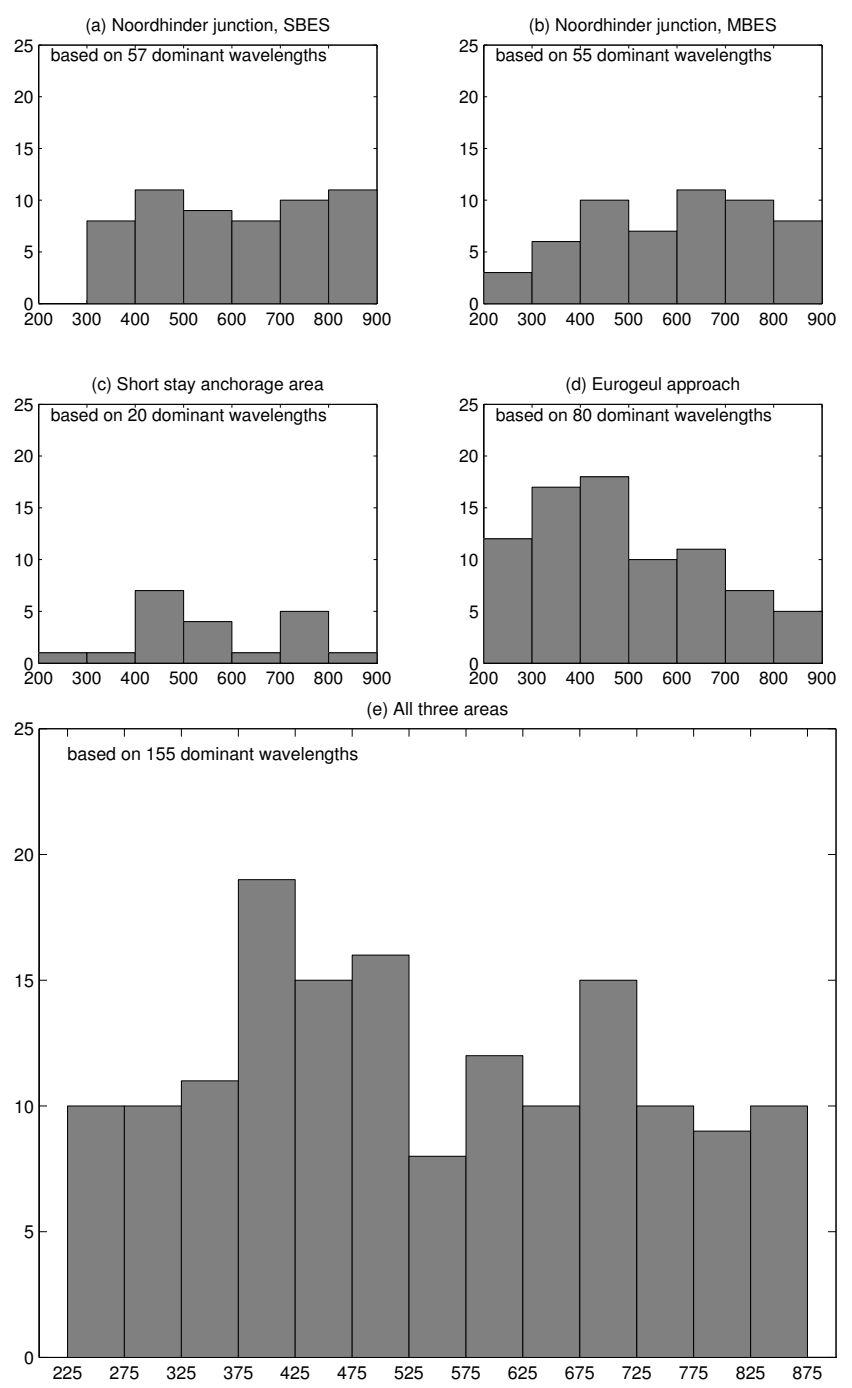

Figure 5.9: Histograms of dominant wavelengths $\underline{L}^{(\mathrm{d})}[\mathrm{m}]$ : (a) for the SBES surveys of the Noordhinder Junction; (b) for the MBES surveys of the Noordhinder Junction; (c) for the Eurogeul Approach area; (d) for the Short Stay anchorage area; (e) for all three areas, in which the MBES results are used for the Noordhinder Junction. Note that we have changed the bin resolution of graph (e). 
the average bed level could have become deeper during the SBES period, but not during the later MBES period.

Dynamics were not detected for the sand wave patterns of the Noordhinder junction, neither from the two-dimensional analyses, nor from the onedimensional analyses. Apparently, the removal of sediment from the highest crests was insufficient to be detected as a decrease in amplitude, but large enough to be detected as a change in bed level. It is shown in Chapter 3 that the procedure is more sensitive to bed level dynamics than sand wave amplitude dynamics, because the Minimal Detectable Biases are smaller for bed level dynamics.

For the Noordhinder Junction, there are no subareas with more than one outlier in bed level $d^{(\mathrm{b})}$ (Figure 5.10). To explain the outliers, we use Figure 5.11. It shows how the outliers are distributed in time. The outliers of the MBES analyses are all due to the last survey, in 2006 (Figure 5.11a). They describe a depth decrease of half a meter, with a surprisingly small deviation between the subareas (Figure 5.11b). The outliers for the SBES analyses are due to either the first or the second survey, in 1988 and 1992. Like the MBES outliers, the outliers of the second survey also describe a depth decrease of half a meter, with a small deviation. The outliers of the first survey, however, describe a depth increase, with a large deviation between the subareas. The outliers in the SBES surveys indicate that the detected trends in bed level are not always linear. The shallower outliers for the most recent MBES survey may indicate that the trend toward deeper bed levels has not only stopped, but even reversed.

\section{Grid node analyses}

In Section 5.4.3, we will compare the results of deformation analysis with the results of trend analysis [Wüst, 2005]. Trend analysis provides for trends in depth at a specific location. To facilitate such a comparison, we now consider the largest upward trend per subarea from the results of the zero-dimensional deformation analysis per grid node. The results are plotted for the four analyzed SBES surveys and for the six analyzed MBES surveys of the Noordhinder Junction, and for the Short Stay anchorage area and the Eurogeul Approach area, in Figure 5.12. As we already have detected a bed level trend in downward direction for the Noordhinder Junction SBES surveys, it is logical that there are hardly any subareas for which a nodal upward trend is detected, see Figure 5.12a. The Noordhinder Junction MBES surveys and the surveys of the other two areas show more upward trends, mostly of the order of a few decimeters a year (Figures 5.12b-c). However, one extreme value is visible in the Northeastern corner of the Noordhinder Junction, for the MBES surveys.

The subarea with the largest upward trend is indicated with an arrow in Figure 5.12b, and shown in Figure 5.13. The isolines represent equal depth, where the darkest lines are the shallowest. It is clear from Figure 5.13 that most of the dynamics are found around the crests. The maximum upward trend is detected for the grid node at $(x, y)=(275 \mathrm{~m}, 75 \mathrm{~m})$. At this node, two outliers are detected in addition to the trend. The dynamics at this node are depicted 

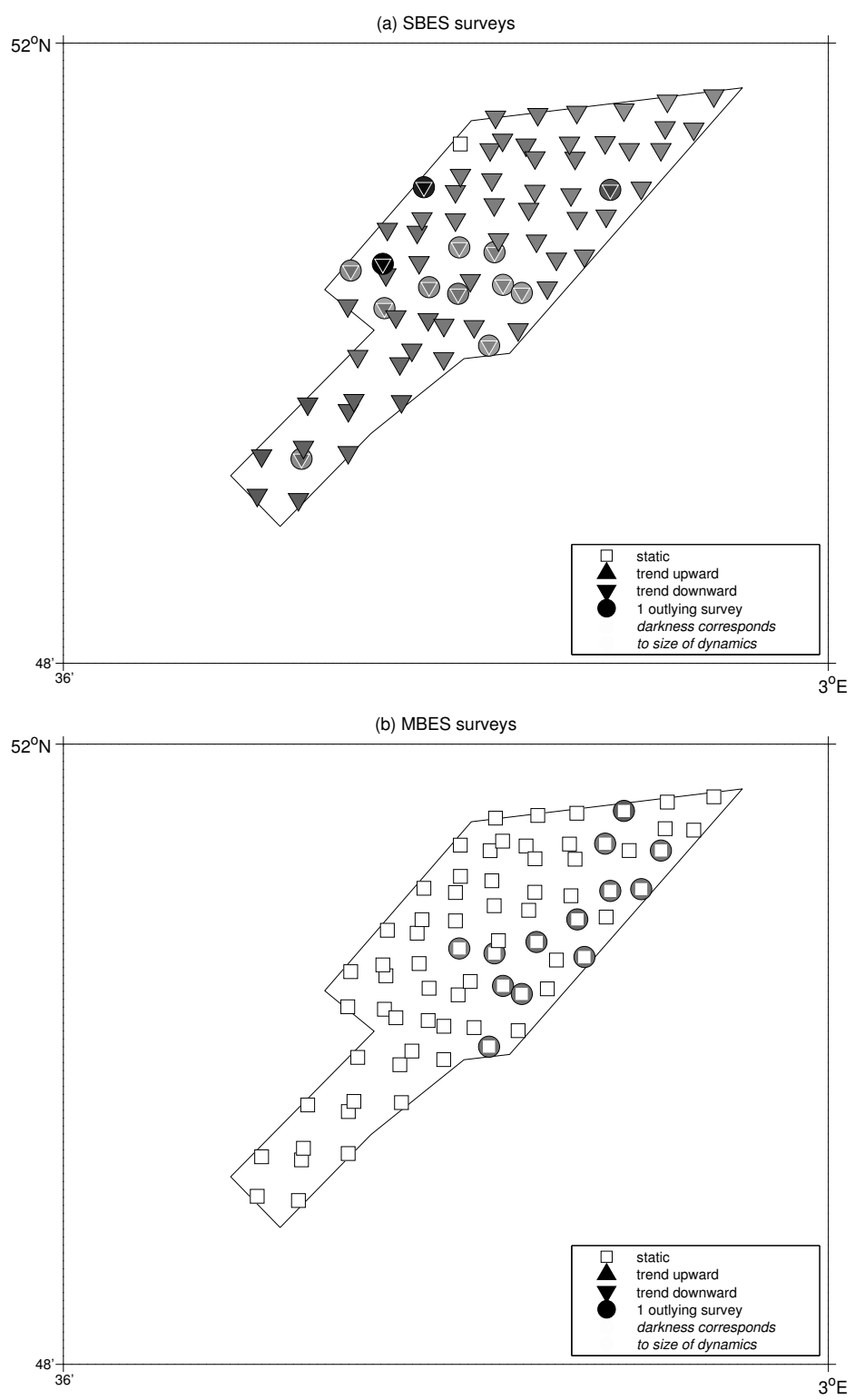

Figure 5.10: Dynamics of bed level $\underline{d}^{(\mathrm{b})}$ for the Noordhinder Junction: (a) SBES surveys; (b) MBES surveys. (The values of the detected downward trends are 0.1 to $0.3 \mathrm{~m} / \mathrm{yr}$ in downward direction, shown by the shade of the triangles; the values of the outlying bed levels are 0.4 to $1.0 \mathrm{~m}$, shown by the shade of the circles. The uncertainties at a $95 \%$ confidence interval of the trends are 0.1 to $0.2 \mathrm{~m} / \mathrm{yr}$, and of the outlying bed levels 0.2 to $0.8 \mathrm{~m}$.) 

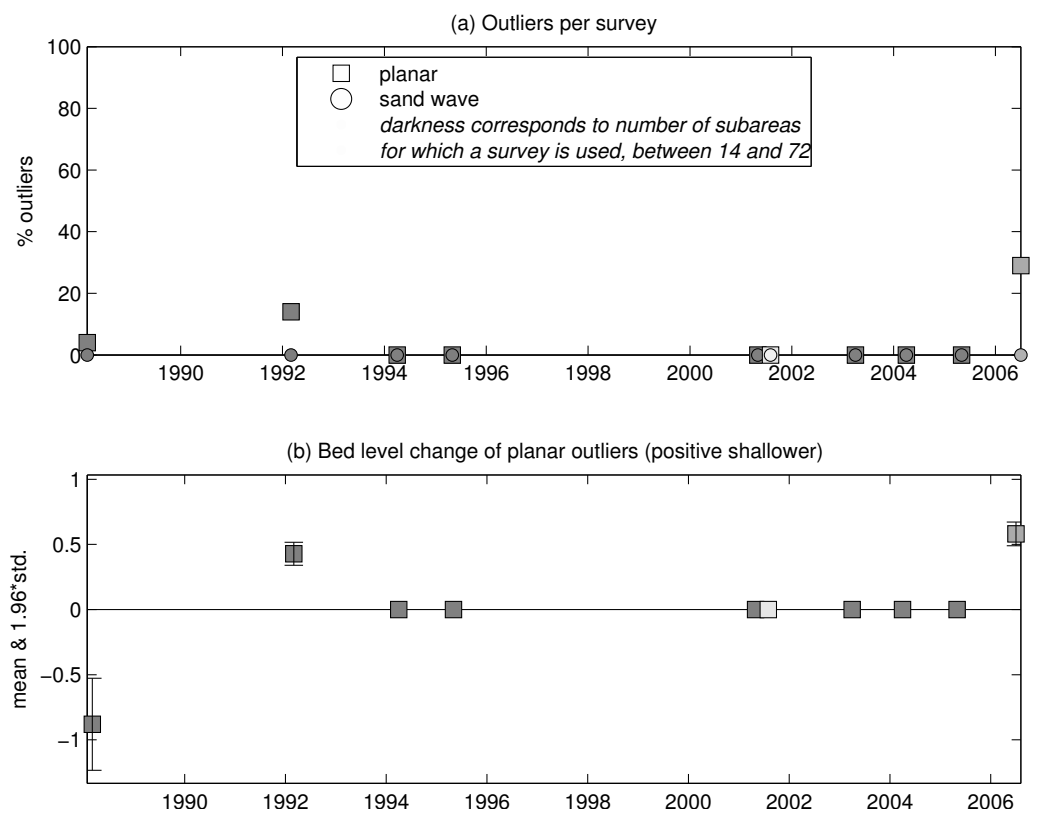

Figure 5.11: Outliers in bed level $\underline{d}^{(\mathrm{b})}$ per survey for the Noordhinder Junction: (a) number of outliers per survey, summed over all subareas; (b) average bed level change and variation in bed level change, for the planar outliers. Figure (a) shows that there are no sand wave outliers, and Figure (b) provides details on the value (squares, positive is shallower) and variation (error bars, at a 95\% confidence level) of the planar outliers. (The shade corresponds to the number of subareas for which a survey is used, between 14 and 72, darker is larger number of subareas.) The analysis period for the four SBES surveys is 1988 to 1995, and for the six MBES surveys 2001 to 2006 .

in Figure 5.14. The detection and subsequent removal of outlying bed level values has caused the detection of a trend, which is only based on the last three bed level values. If, instead, the last two surveys would have been qualified as outliers, a linear trend in the other direction would have been found instead. This example shows that the detection of several outliers in combination with a trend indicates that the true trend is nonlinear, because the rate of change for depth changes in time. A cause for the nonlinear trend could be local dynamics of the nearby crest.

\subsubsection{Discussion: sand wave migration and trend analysis in literature}

Sand wave migration in the Selected Track and Noordhinder regions was previously studied by Van Dijk et al. [2008] and Knaapen [2005], and trends in depth of those areas by Wüst [2005] (Figure 5.2). The method used by Knaapen [2005] 

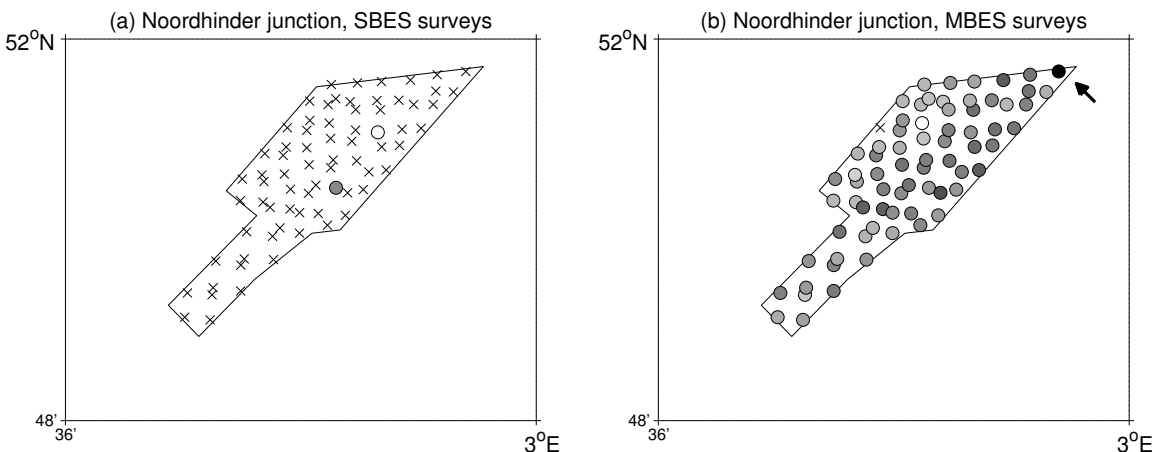

(c) Short stay anchorage area and Eurogeul approach area

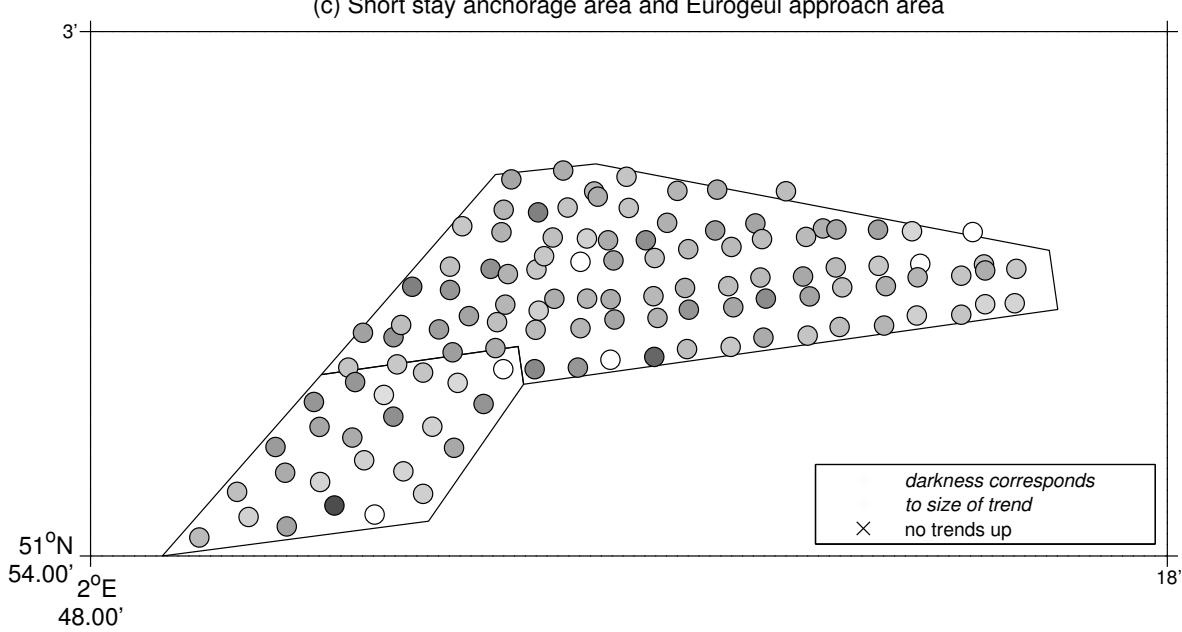

Figure 5.12: Maximum upward trends $\dot{d}^{(\mathrm{b})}$ of the zero-dimensional grid node analysis for: (a) the SBES surveys of the Noordhinder Junction; (b) the MBES surveys of the Noordhinder Junction; (c) the Eurogeul Approach area and the Short Stay anchorage area. (The maximum upward nodal trend per subarea is 0.0 to $1.2 \mathrm{~m} / \mathrm{yr}$, and the average $0.3 \mathrm{~m} / \mathrm{yr}$. The shade corresponds to the value of the maximum upward nodal trend per subarea. The uncertainties at a $95 \%$ confidence level are $0.3 \mathrm{~m} / \mathrm{yr}$.) A cross indicates there are no upward nodal trends for that subarea. The arrow indicates the subarea shown in detail in Figure 5.13.

derives sand wave migration from automatically detected crest and trough positions. This way, it is possible to study sand wave asymmetries and irregularities. Van Dijk et al. [2008] estimate morphological parameters like crest and trough position per survey, from which they estimate their change in time. They are able to estimate changes in wavelength, which our analysis does not allow for. The estimated migration rates of Van Dijk et al. [2008] and Knaapen [2005] are of the order of several decimeters a year (Table 5.1). 


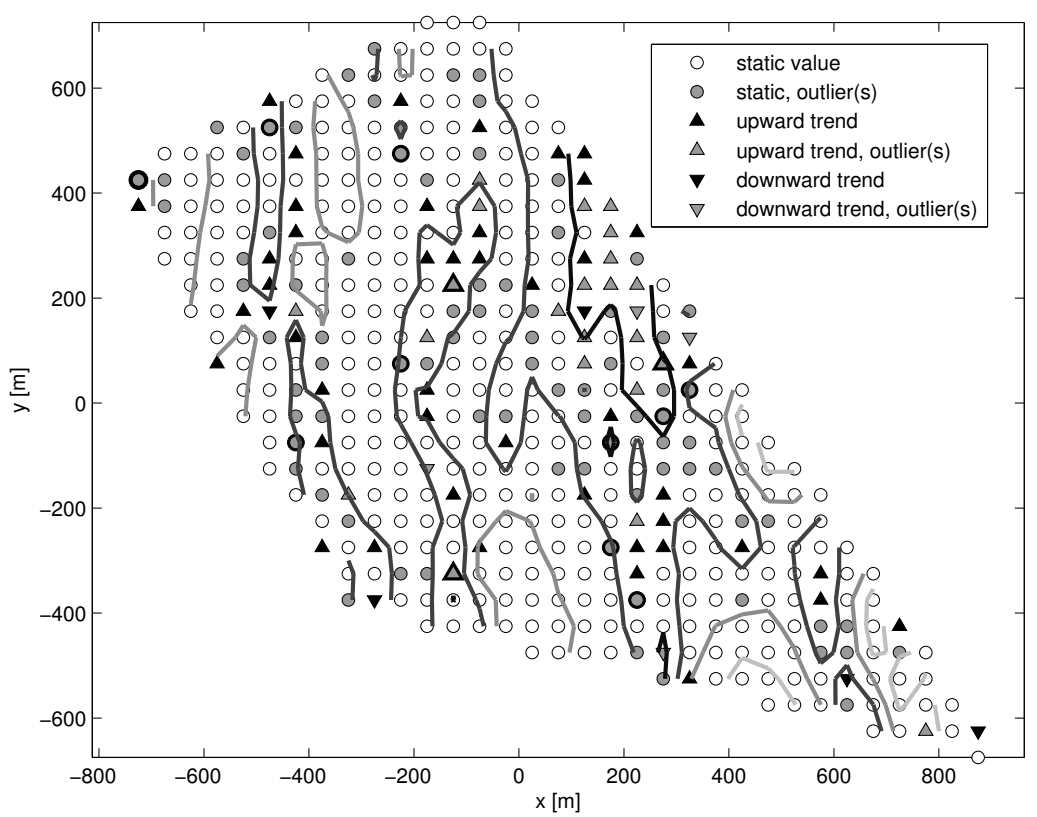

Figure 5.13: Detailed results of the zero-dimensional grid node analysis for the subarea in the Northeastern corner of the Noordhinder Junction, which shows the largest maximum in Figure 5.12. The dynamics in depth $\underline{d}^{(\mathrm{b})}$ per node are shown, with a maximum upward trend $\underline{\dot{d}}^{(\mathrm{b})}$ of $1.2 \mathrm{~m} / \mathrm{yr}$ at $(x, y)=(275 \mathrm{~m}, 75 \mathrm{~m})$. At this location, a symbol for an upward trend in combination with two outliers (thick edges) is visible. Also, isolines of equal depth are shown to indicate the sand wave pattern. (The darkest lines are the shallowest.) The Dynamics at this node are shown in Figure 5.14.

However, crest and trough detection is prone to positioning and depth errors. Depth errors potentially play a significant role in the detection of local minima and maxima, and positioning errors in their location. Deformation analysis, on the other hand, uses a grid of depth values on fixed positions, thereby strongly reducing the dependence on errors in crest and trough identification. The results of the one- and two-dimensional deformation analyses did not display any sand wave dynamics, and we conclude that the migration rates are not significant.

Wüst [2004] developed a method to estimate upward trends in depth at specific locations, termed trend analysis, see also Lindenbergh et al. [2007]; Knaapen et al. [2005]. Results for the areas around the Eurogeul approach are given in Wüst [2005] and in Figure 5.15. Per subarea of a few square kilometres, the maximum upward trend is shown. A comparison with the zerodimensional results per grid node of Figure 5.12 shows that the maximum results of deformation analysis are larger. This is explained by a number of reasons. 


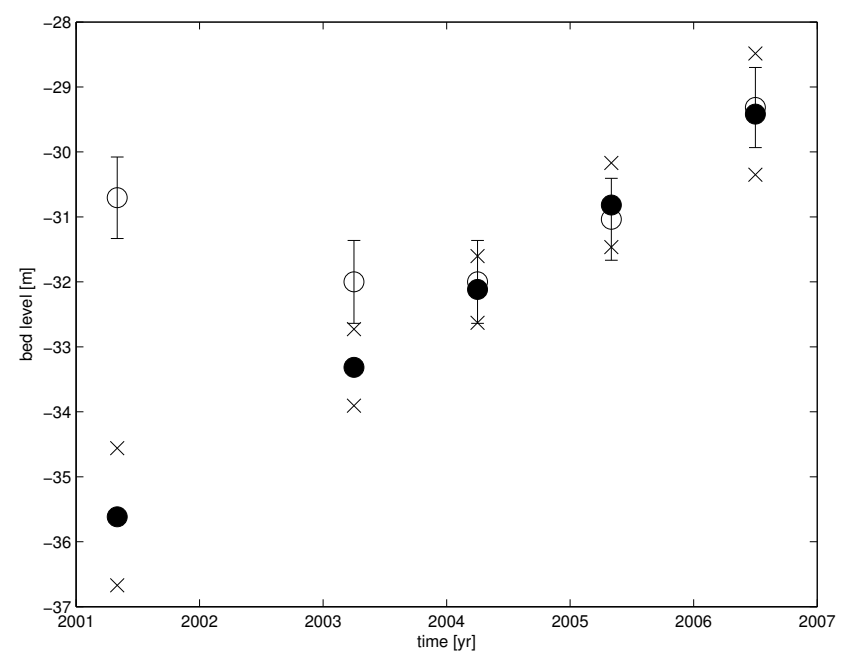

Figure 5.14: The dynamics of the node at $(x, y)=(275 \mathrm{~m}, 75 \mathrm{~m})$. The white circles are the nodal depth values per survey, and the black circles indicate the linear trend $\underline{\dot{d}}^{(\mathrm{b})}$, without the two outliers for the first two surveys. The vertical lines and the crosses indicate the $95 \%$ confidence intervals of the nodal depth values and the linear trend, respectively.

1. Our deformation analysis allows for a combination of a linear trend and outliers. As already concluded in Section 5.4.2, a detected linear trend in the presence of several outliers indicates nonlinear behaviour instead, and the linear estimate does not represent a constant rate of depth change. The large trend values of deformation analysis may be the results of the qualification of values that cause a reduction of the size of the trend as outliers, which subsequently have no impact anymore on the estimation of the linear trend.

2. A trend analysis estimates the trend at the moment of the last survey, while deformation analysis, in the absence of outliers, estimates an average trend over the complete time interval of all surveys.

3. The used surveys are not the same for the two types of analysis.

4. The results of the trend analyses are valid for the shallowest locations within the three areas. Often, the large upward trends in the bed levels of the deformation analysis results are positioned on deeper parts of the three areas.

The largest maximum of deformation analysis in Figure 5.12c is larger than the largest maximum of the trend analysis $(1.2 \mathrm{~m} / \mathrm{yr}$ versus $0.3 \mathrm{~m} / \mathrm{yr})$, but the average maxima are roughly similar $(0.3 \mathrm{~m} / \mathrm{yr}$ versus $0.2 \mathrm{~m} / \mathrm{yr})$. The upward nodal 


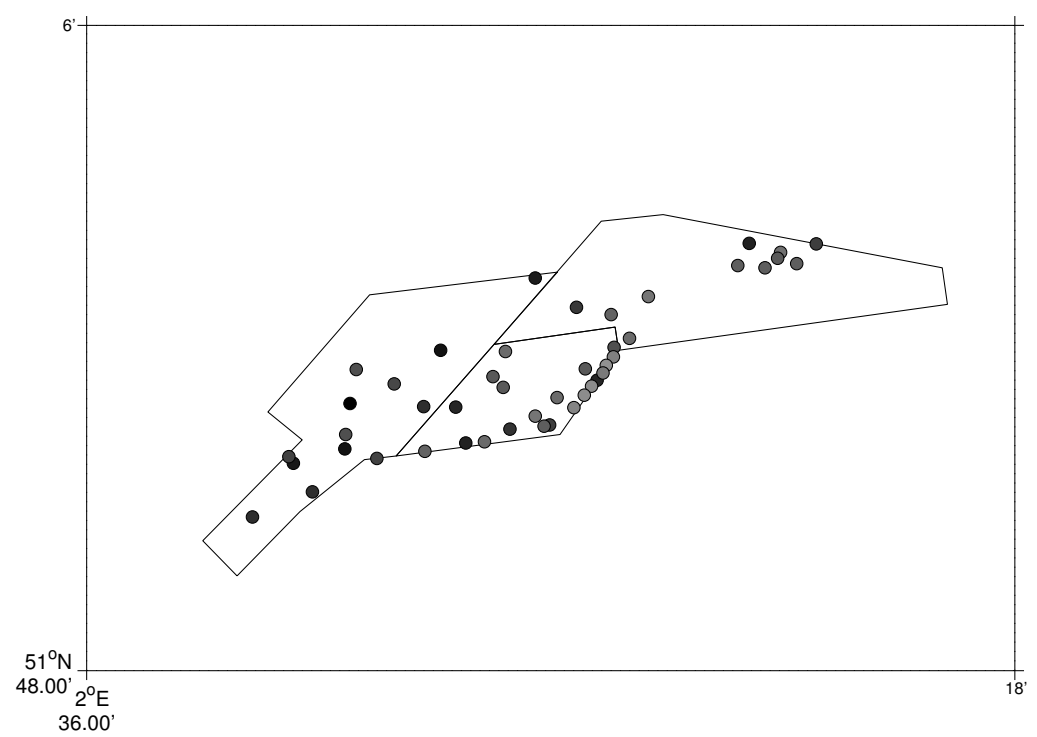

Figure 5.15: Upward trend estimates for depth by Wüst [2005]. (The maximum upward nodal trend per subarea is 0.1 to $0.3 \mathrm{~m} / \mathrm{yr}$, and the average $0.2 \mathrm{~m} / \mathrm{yr}$. The shade corresponds to the value of the maximum upward nodal trend per subarea.)

trends are estimated by the deformation analysis procedure with an uncertainty of $0.3 \mathrm{~m} / \mathrm{yr}$ at the $95 \%$ confidence level. This makes the difference between the results of both methods negligible, except for the very large $1.2 \mathrm{~m} / \mathrm{yr}$ trend, shown in Figure 5.13.

\subsection{The region West of IJmuiden: sand wave migration}

The IJgeul Approach area and IJgeul Approach anchorage area show a complicated sand wave pattern, which potentially endangers shipping because of its shallow depth in combination with expected sand wave dynamics. In this region, there are no analyses available in literature on sea floor dynamics. The available surveys are listed in Appendix 5.A. Both the areas in the region West of IJmuiden are shown in Figure 5.16. Although these areas have a guaranteed depth, no dredging history is available [Stolk, personal communication].

\subsubsection{Spatial and temporal characterizations}

An overview of the available surveys is given in Figure 5.17a, the spatial characterizations in Figure 5.17a-c, and the dynamics using a two-dimensional deformation analysis in Figure 5.18 for bed level and in Figure 5.19a-b for sand wave dynamics. The results for the areas around the IJgeul Approach are similar to the results for the areas around the Eurogeul Approach: the distribution of the 


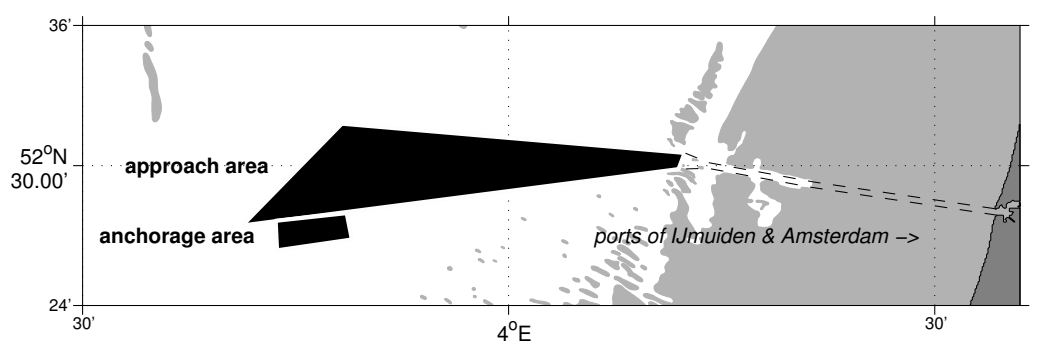

Figure 5.16: Location of the IJgeul Approach area and IJgeul Approach anchorage area.

wavelengths has two peaks, the largest one at around $400 \mathrm{~m}$, and a second one at around $700 \mathrm{~m}$, see Figure 5.20.

The DIGIPOL-directions are uniform over the area, but the wavelengths are not: the sand wave pattern is in fact more complicated than the spatial characterizations can deal with. In some cases, this leads to the rejection of the sand wave extension, but more often a sand wave is found, for which the wavelength strongly depends on more or less random properties, like the locations of the defined boundaries of the subarea, and the presence of sand wave irregularities, i.e. bifurcations and finite-length crests.

The central part of the area appears to become deeper over time, see Figure 5.18. The Western and Eastern parts show outliers. Although no dredging history is available, depth maintenance may explain the bed level dynamics. Many of the sand wave patterns show a trend, which is by definition detected for both amplitude change and migration. The detected trends in amplitude are very small, though: the maximum absolute rate in amplitude factor is $3 \%$ a year. The detected migration rates are several meters a year, all in Northeastern direction. The maximum rate is $7 \mathrm{~m} / \mathrm{yr}$, which is of the same order as found for the Maas West anchorage area (Chapter 4), also located relatively close to the coast.

The results show several outliers in bed level (Figure 5.18), and only one for the sand wave (Figure 5.19a-b). The June 2003 survey caused the single sand wave outlier, for which the amplitude is $36 \%$ smaller, and the position has changed $26 \mathrm{~m}$ to the Northeast. Their $95 \%$ confidence intervals are $37 \%$ and 18 $\mathrm{m}$, respectively, which shows that this outlier is only significant for the position of the pattern.

Most bed level outliers originate from the first or second survey. These surveys are outlying for $16 \%$ and $20 \%$ of the subareas, respectively. The outlier values are on average $0.3 \mathrm{~m}$ shallower and $0.3 \mathrm{~m}$ deeper, respectively, with deviations of up to $0.1 \mathrm{~m}$. The typical uncertainty of an estimated bed level outlier is $0.2 \mathrm{~m}$, at a $95 \%$ confidence level. Although Figure 5.18 shows two 

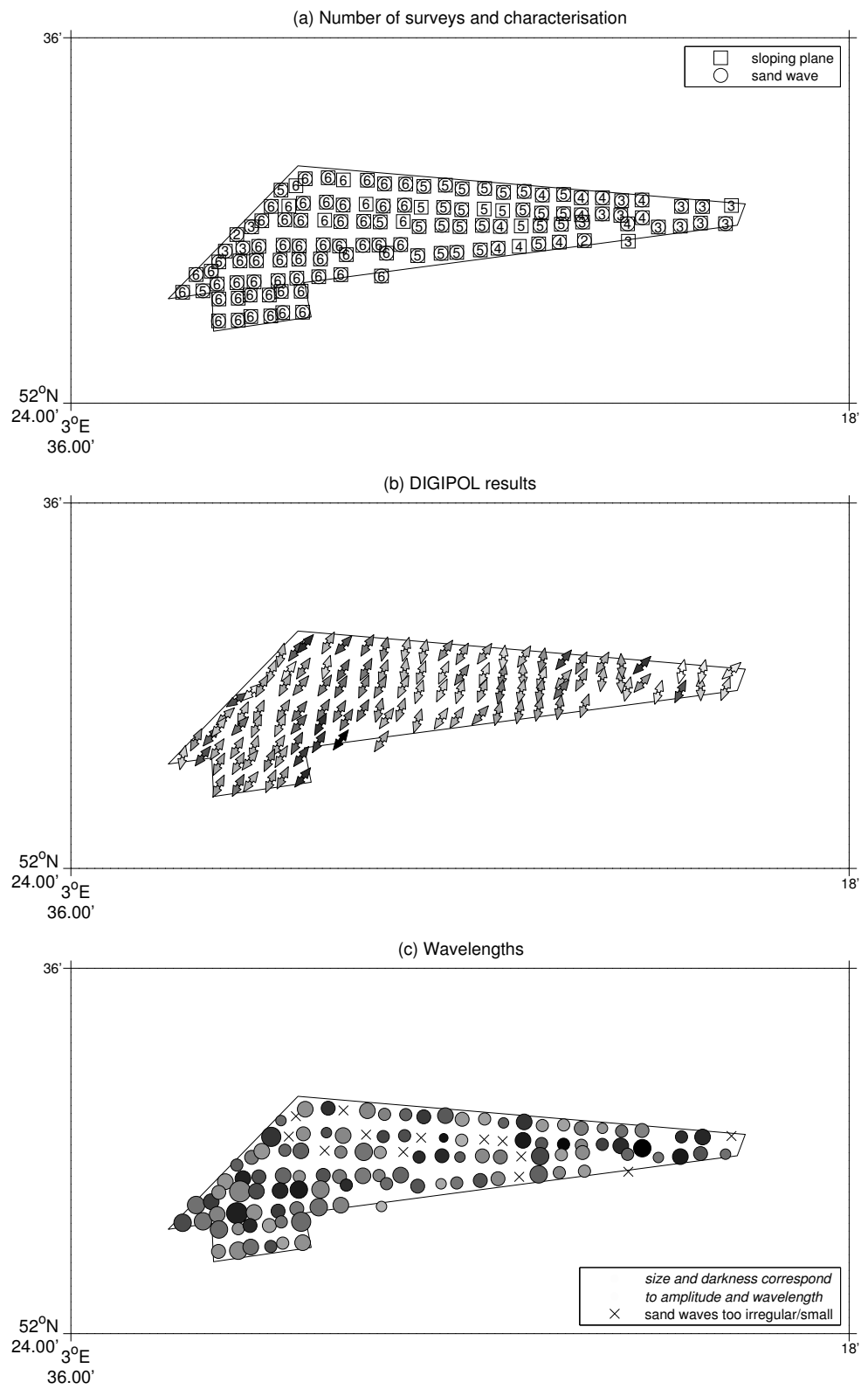

Figure 5.17: Spatial results for the IJgeul Approach area: (a) number of surveys and characterization; (b) DIGIPOL directions $\underline{\theta}^{(\mathrm{x})}$ and quotients $\max (\underline{v}) / \min (\underline{v})$ (quotients 1.6 to 6.0 , darker is larger) and (c) dominant wavelengths $\underline{L}^{(\mathrm{d})}$ (between 234 and $852 \mathrm{~m}$, shown by the shade of the circles, darker is larger wavelength) and amplitudes $\underline{A}$ (between 0.3 and $1.5 \mathrm{~m}$, shown by size of the circles, larger is larger amplitude). 


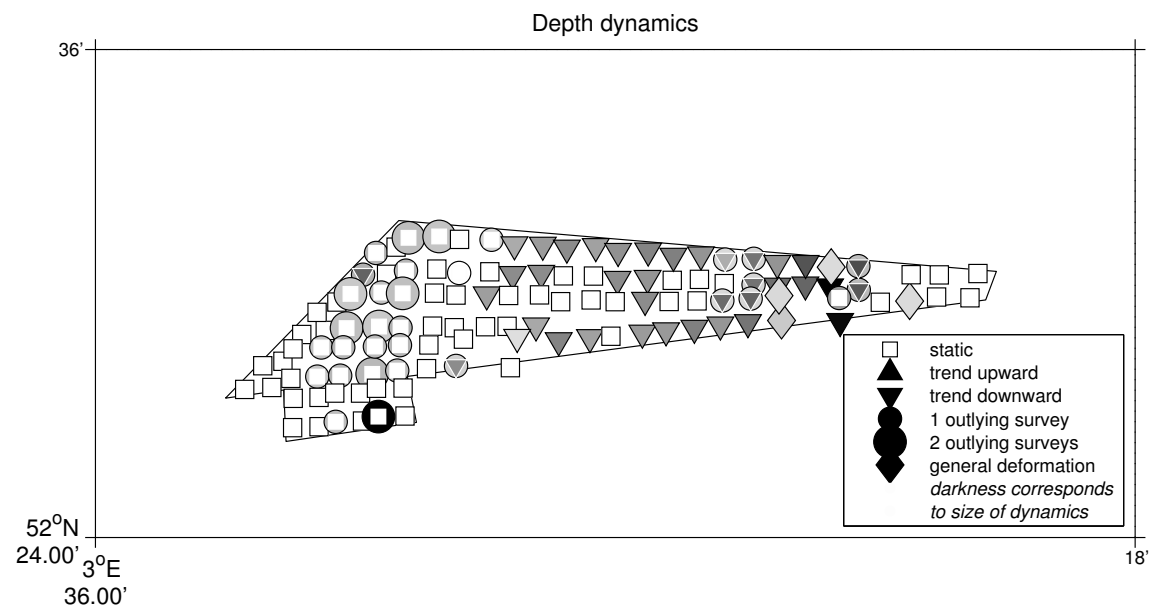

Figure 5.18: Dynamics of bed level $\underline{d}^{(\mathrm{b})}$ for the IJgeul Approach area. (The values of the detected downward trends are between $0.01 \mathrm{~m} / \mathrm{yr}$ and $0.03 \mathrm{~m} / \mathrm{yr}$ in downward direction, shown by the shade of the triangles; the absolute values of the outlying bed level values are between 0.2 and $1.1 \mathrm{~m}$, shown by the shade of the circles and diamonds. The uncertainties at a $95 \%$ confidence interval of the trends are 0.01 to $0.08 \mathrm{~m} / \mathrm{yr}$, and of the outlying bed levels 0.1 to $0.3 \mathrm{~m}$.)

clear parts of the area in which the bed level outliers are present, neither of the two surveys with outlying bed levels corresponds to such a specific part.

\subsubsection{Correlations between migration and morphology}

Analogously to Chapter 4, we try to find correlations between the migration rates $\dot{\xi}^{\prime}$ on one hand, and the bed level $\underline{d}^{(\mathrm{b})}$ and a migration predictor $\dot{\xi}^{(\mathrm{p})}$ on the other hand. The migration predictor was developed by Knaapen [2005], and is based on shape information of a sand wave pattern only. Using these correlations, we aim to confirm previously studies by Van Dijk and Kleinhans [2005] for bed level, and by Knaapen [2005] for the migration predictor. We calculate an adapted version (Chapter 4 ) of the migration predictor as:

$$
\underline{\dot{\xi}}^{(\mathrm{p})}=c^{(\mathrm{a})} \underline{L}^{(\mathrm{d}) 2} \underline{\sigma}^{(\mathrm{m}) 2} / \underline{\sigma}_{d}^{(\mathrm{s}) 3},
$$

based on dominant wavelength $\underline{L}^{(\mathrm{d})}$, morphological variance $\underline{\sigma}^{(\mathrm{m}) 2}$, and spatial depth variation $\underline{\sigma}_{d}^{(\mathrm{s})}$ (Chapter 4$)$. The adapted calibration constant $c^{(\mathrm{a})}$ has a value of $6.49 \cdot 10^{-5} \mathrm{yr}^{-1}$. The linear correlations are:

$$
\underline{\dot{\xi}}^{\prime}=a_{d} \underline{d}^{(\mathrm{b})}+b_{d}
$$



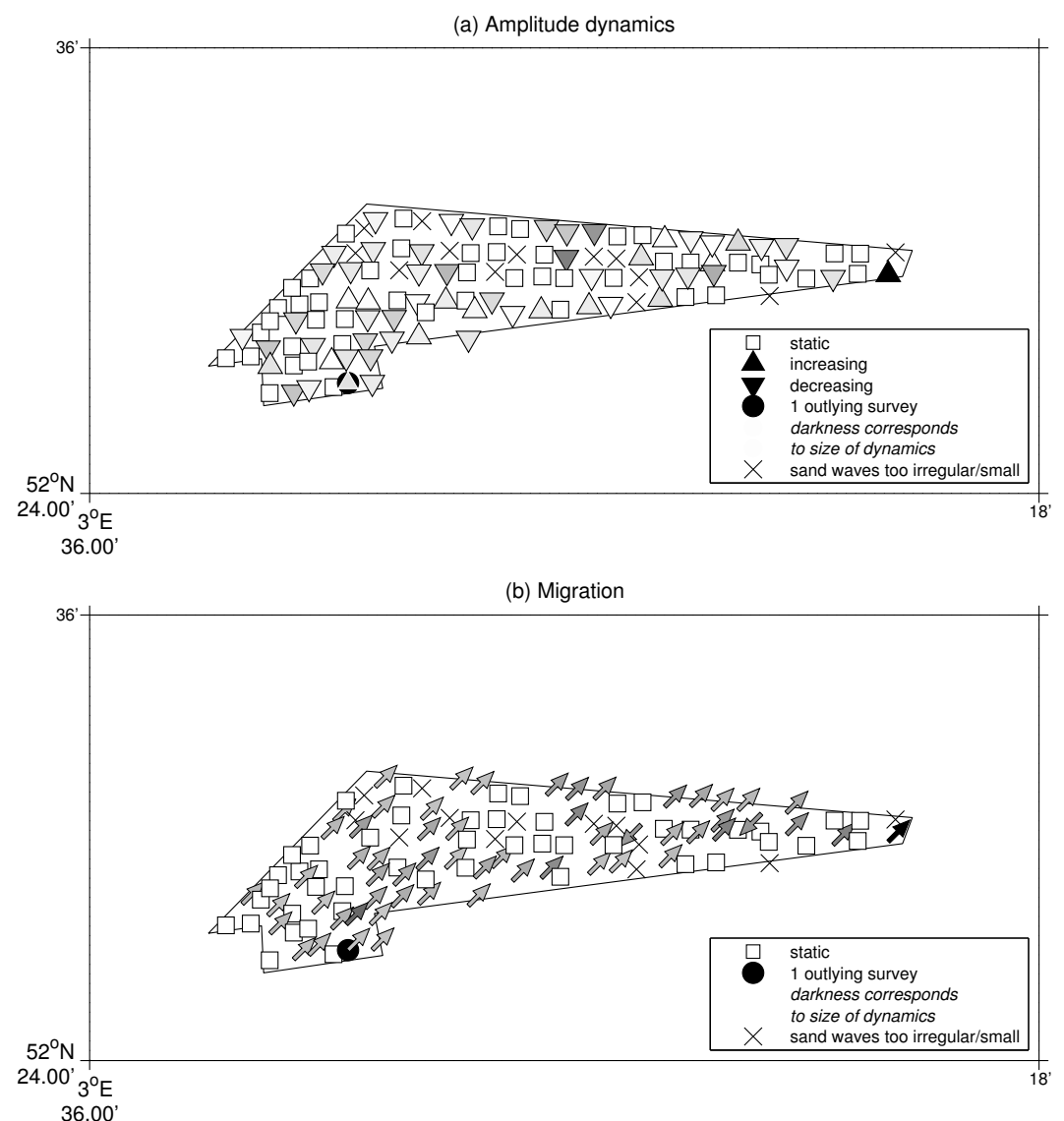

Figure 5.19: Results of sand wave dynamics for the IJgeul Approach area: (a) dynamics of amplitude factor $\underline{A}^{\prime}$ (The maximum trend values are a $2 \% / \mathrm{yr}$ decrease and a $3 \%$ increase, the absolute values of the trends are shown by the shade of the triangles; the outlier is a $36 \%$ decrease. The uncertainties at a $95 \%$ confidence interval of the trends are 1 to $3 \% / y r$, and of the outlying amplitude factor $37 \%$.); and (b) dynamics of crest position $\underline{\xi}^{\prime}$ (The maximum trend in Northeastern direction is $7 \mathrm{~m} / \mathrm{yr}$, and $3 \mathrm{~m} / \mathrm{yr}$ in Southwestern direction, the absolute values of the trend values are shown by the shade of the arrows; the outlier represents a position difference of $26 \mathrm{~m}$. The uncertainties at a $95 \%$ confidence interval of the trends are 1 to $5 \mathrm{~m} / \mathrm{yr}$, and of the position outlier $18 \mathrm{~m}$.) 


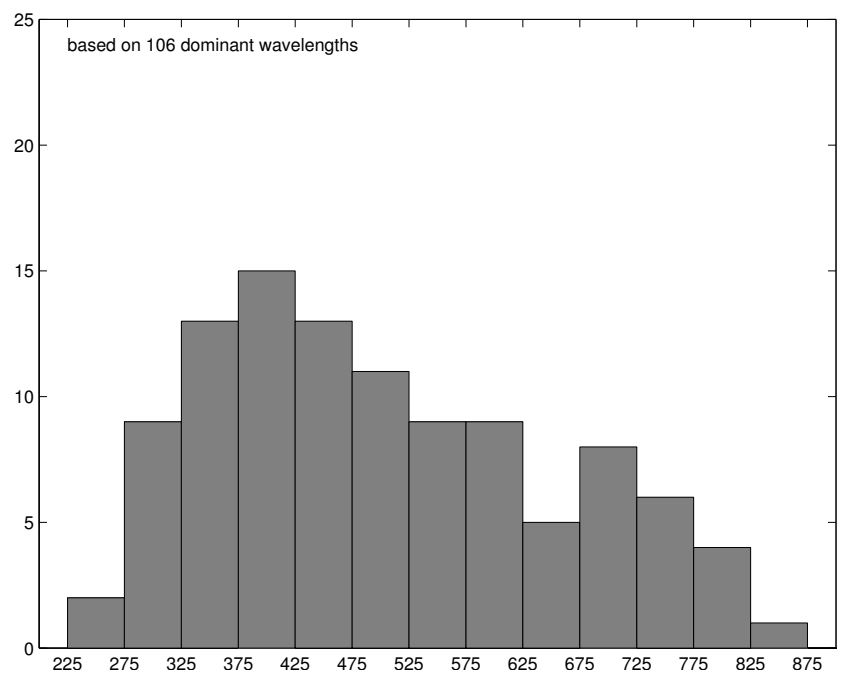

Figure 5.20: Histogram of the dominant wavelength $\underline{L}^{(\mathrm{d})}[\mathrm{m}]$ of the IJgeul Approach area. The search interval and bin resolution are equal to Figure 5.9e.

Table 5.5: Correlation estimates $\underline{a}, \underline{b}$ and their uncertainties expressed as $95 \%$ confidence limits, given as estimate \pm uncertainty, for migration rate $\underline{\dot{\xi}}^{\prime}$ with bed level $\underline{d}^{(\mathrm{b})}$ and migration predictor $\dot{\xi}^{(\mathrm{p})}$. These estimates are used in equations 5.2 and 5.3. Also, correlation coefficients $\underline{\rho}$ are given.

\begin{tabular}{cccc}
\hline \hline & $\underline{a}$ & $\underline{b}$ & $\underline{\rho}$ \\
\hline$\underline{d}^{(\mathrm{b})}$ & $-0.16 \pm 0.11 \mathrm{yr}^{-1}$ & $6.1 \pm 2.6 \mathrm{~m} / \mathrm{yr}$ & -0.27 \\
$\underline{\underline{\xi}}^{(\mathrm{p})}$ & $-0.01 \pm 0.16$ & $2.0 \pm 0.4 \mathrm{~m} / \mathrm{yr}$ & -0.01 \\
\hline \hline
\end{tabular}

and

$$
\underline{\dot{\xi}}^{\prime}=a_{\xi} \underline{\dot{\xi}}^{(\mathrm{p})}+b_{\xi}
$$

Estimates of the coefficients $\underline{a}, \underline{b}$ for the region West of IJmuiden are given in Table 5.5. In contrast to the Maas West anchorage area (Chapter 4), hardly any correlation is found with bed level and shape information. Relevant differences between the two areas are the smaller depth of the Maas West area (16-23 m instead of 21-28 m), and the smaller wavelengths (values up to $360 \mathrm{~m}$ instead of values up to $852 \mathrm{~m}$ ). 


\subsection{The region North of Terschelling: a flat sea floor}

The Terschelling-German Bight traffic separation scheme (TE-TSS) contains a shallow and rather flat part North of Terschelling (Figure 5.21). In the deformation analysis procedure, we disable the sand wave extension for this region, but allow for the slope extension. This is done because residual systematic errors in the track direction may result in the detection of a spurious sand wave pattern. The track direction coincides with the direction of the traffic separation scheme. Figure 5.21a shows that systematic measurement errors dominate the residuals, as the DIGIPOL-directions of highest variation are approximately perpendicular to the track direction.

We analyze four surveys of this area, measured between 1996 and 2005 (Appendix 5.A). The absence of rhythmic bed forms means that the characterizations of the subareas by a horizontal or sloping plane is close to the actual shape of the sea floor. Therefore, it turns out that most of the overall tests are accepted, for the spatial characterization of the two-dimensional analyses (step 1). Thus, it is not necessary to increase the variances and covariances with morphological components by the LSVCE procedure, and as a consequence the confidence intervals for the depth values in the temporal characterization (step 2) are smaller than for the other areas. This, in turn, makes the temporal estimation accurate, yet sensitive to extensions, see Figure 5.21b. The deformation analysis procedure accepts many extensions for the bed level, often in the order of a decimeter only, resulting in linear trends of a few centimeters a year only.

As only four surveys are available, the detection of more than one outlier to the linear trend means that there are as many bed level parameters as there are surveys, and the subarea is qualified as generally deforming. This is denoted by the diamond-shaped symbols in Figure 5.21. The survey showing a single outlying depth always is the 2002 survey, which is on average $0.3 \mathrm{~m}$ deeper than the upward trend value for that survey would have been, with a deviation up to $0.1 \mathrm{~m}$.

\subsection{Discussion}

The results of the considered regions were discussed in the previous Sections. Here, we discuss the methodical and the morphological question of the Introduction.

\subsubsection{Methodical}

The application of deformation analysis to areas on the BNLCS shows results that reveal diverse dynamics, with an associated uncertainty. To obtain these results, it is necessary to carefully set several input parameters, and use limitations for the amount of data used. The input parameters are the error variance and covariance models, the levels of significance, the wavelength search interval, and the presence of tidal sand waves. Therefore, detailed knowledge of the meta-data of the used surveys is necessary, as well as some prior knowledge of the sea floor of the subareas. The definition of subareas of a few square 

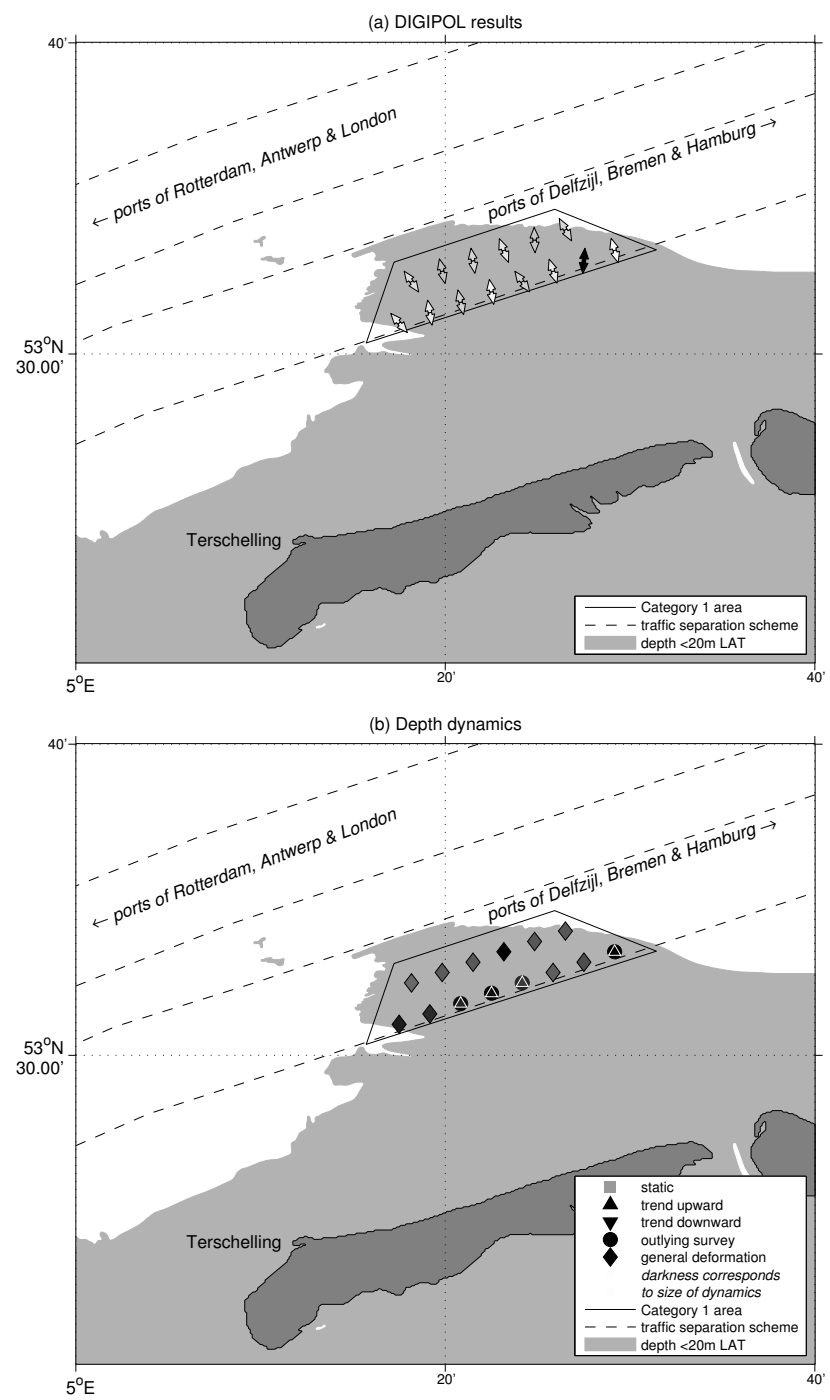

Figure 5.21: Results for the shallow part of the TE-TSS: (a) DIGIPOL directions $\underline{\theta}^{(\mathrm{x})}$, and quotients $\max (\underline{v}) / \min (\underline{v})$ (between 1.4 and 4.7 , darker is larger) and (b) dynamics of bed level $\underline{d}^{(\mathrm{b})}$. (The values of the upward trends are between 0.03 and $0.05 \mathrm{~m} / \mathrm{yr}$, shown by the shade of the triangles. The bed level variations due to outliers or general deformation are up to $0.37 \mathrm{~m}$, shown by the shade of the circles and diamonds. The uncertainties at a $95 \%$ confidence interval of the trends are 0.01 $\mathrm{m} / \mathrm{yr}$, and of the outlying bed levels $0.1 \mathrm{~m}$.) 
kilometers, in combination with the definition of grids with a grid spacing of at least $50 \mathrm{~m}$, gives results that are sufficiently detailed in space, and still not too computation-intensive. To keep the computations feasible, the number of surveys needs to be limited to less than about eight, for such a grid.

Still, some methodical artifacts appear in some cases, especially if a trend is detected in combination with several outliers. In that case, the interpretation could only be that a nonlinear trend is present, and the estimated dynamics are not useful. Further, the detection of sand waves suffers from the choice of limits of the search interval. Areas that have several patterns with different wavelengths superimposed show the limited usability of a search procedure with a single dominant wavelength.

\subsubsection{Morphological}

The analyses of the regions Noordhinder and West of IJmuiden reveal a wide variety of dominant wavelengths. Two wavelengths, of around 400 and $700 \mathrm{~m}$, seem to dominate in both these regions. This contradicts the wavelengths of about 250 and $550 \mathrm{~m}$ reported in the literature.

An overview of sand wave behaviour on the BNLCS is given in Figure 5.22, including the behaviour of the Maas west area, described by Chapter 4. The sand wave regions West of Rotterdam and West of IJmuiden are located closer to the coast than the other two regions that have sand waves. They also are the two regions that reveal sand wave migration, of up to $7.5 \mathrm{~m} / \mathrm{yr}$. It is interesting to note that the region West of Rotterdam seems to have migration rates that are correlated with depth and shape information, while the region West of IJmuiden does not show this correlation.

The two sand wave regions near the coast show migration and have estimated bed levels $\underline{d}^{(\mathrm{b})}$ of 20 to $25 \mathrm{~m}$, while the regions away from the coast do not show migration and are 35 to $40 \mathrm{~m}$ deep. This observation confirms the results of Van Dijk and Kleinhans [2005], who found a similar correlation for other areas in the Southern North Sea. A more complete evaluation of the morphological properties of the regions would also include a comparison with a process-based model, using hydrodynamic information and sediment properties. Such an evaluation has been done for the Maas West area Chapter 4.

\subsection{Conclusion}

Deformation analysis was successfully applied to bathymetric surveys of a sandy seafloor in various regions on the BNLCS, even in the presence of complicated sand wave patterns. The bed level dynamics of the five analyzed regions are limited to several decimeters over the past two decades, except for the zerodimensional results. In this case, the largest upward trends and outliers are usually due to sand wave migration. Large dynamics are absent, except for migration of the sand waves relatively close to the North and South Holland coasts. Migration rates correlate with bed level and shape information for only one of those two regions. These dynamics should result in a higher resurvey 


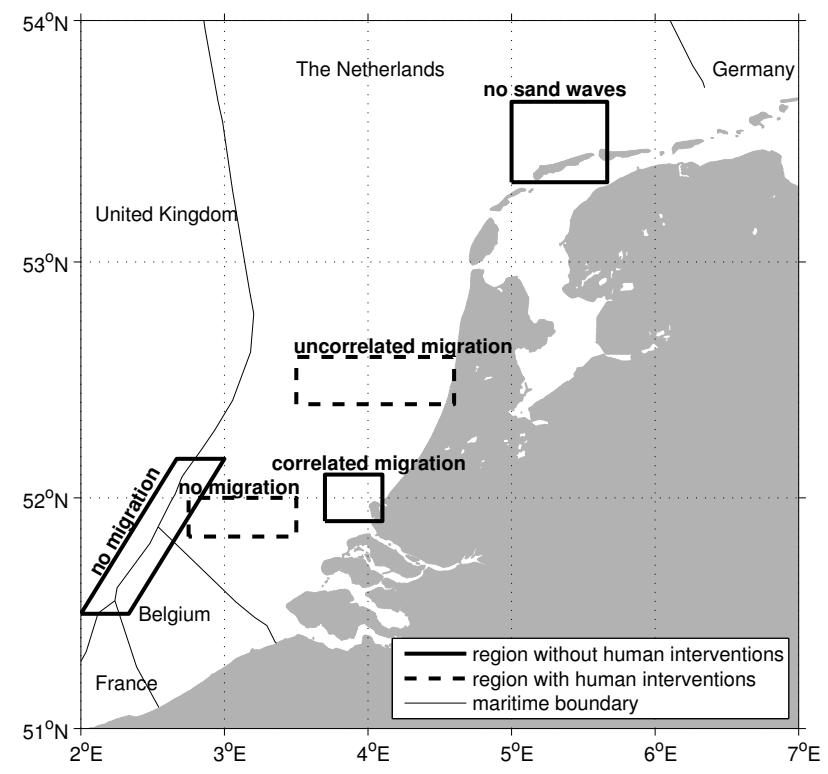

Figure 5.22: Overview of sand wave characteristics for analyzed regions on the Belgian and Netherlands Continental Shelf.

frequency for shallower sand wave areas, than for deeper sand wave areas or areas without a sand wave pattern. No generic conclusions on the impact of human interventions can be drawn, because sand wave dynamics in the two areas with a maintained depth differ from each other.

\section{Appendices}

\section{A Overview of used surveys}

All survey data used are given in the geodetic datum WGS84 in combination with a UTM31-projection. The data are reduced to Lowest Astronomical Tide. Only surveys are used for which the data are available on the highest resolution, i.e. each $5 \times 3 \mathrm{~m}$ bin is filled, in track direction. All surveys are available in meters with two decimals, horizontally and vertically. All gross errors were removed from the surveys, and approved by the commanding officer and the Hydrographic Office of the RNLN.

Operational specifications of the surveys are given in the Tables 5.7 and 5.8. The track direction is decided by the commanding officer of the survey vessel, and can therefore vary between surveys. As a constant direction is not possible, due to e.g. traffic, the track direction is estimated from the data up to the 
Table 5.6: Overview of survey systems used for each survey. Operational details of the surveys are given in the Tables 5.7 and 5.8.

\begin{tabular}{cl}
\hline \hline system code & survey system \\
\hline 1 & Hyperfix, pressure gauges \\
2 & DGPS SA on, pressure gauges \\
3 & DGPS SA on, PreMo \\
4 & DGPS SA off, pressure gauge \\
5 & DGPS SA off, PreMo \\
\hline \hline
\end{tabular}

nearest five degrees. The first day of the month in which the survey started is used as the survey moment.

The technical specifications are given in Table 5.6. The presence of Selective Availability is indicated, which is a deliberate degradation of the GPS signals. As differential GPS (DGPS) is used, the impact of SA is not large. The differential reference station used is either part of the IALA chain, or the VeriPos chain. The older surveys use the terrestrial Hyperfix system, which gives the same order of positioning uncertainty as DGPS with SA, i.e. in the order of $10 \mathrm{~m}$. DGPS without SA has an uncertainty of a few meters. The water level reduction method is either based on measurements from pressure gauges placed on the sea floor for the duration of the survey, or from permanent gauges in combination with the PreMo prediction model. 
Table 5.7: Overview of SBES surveys, including survey track spacing $h^{(\mathrm{t})}$, survey track direction East of North $\theta^{(\mathrm{t})}$, and used survey systems for horizontal and vertical reference. The area codes are given in Table 5.2, and the system codes in Table 5.6.

\begin{tabular}{|c|c|c|c|c|}
\hline survey code & area code & start & $h^{(\mathrm{t})} \theta^{(\mathrm{t})}$ & system code \\
\hline HY69 & NH1 & Mar. 1988 & $50 \mathrm{~m} 30^{\circ}$ & 1 \\
\hline HY71 & WIJ1-2 & Mar. 1990 & $50 \mathrm{~m} 45^{\circ}$ & 1 \\
\hline HY91022 & ST1: Twin & Jan. 1991 & $50 \mathrm{~m} 35^{\circ}$ & 1 \\
\hline HY92035 & NH1 & Mar. 1992 & $100 \mathrm{~m} 30^{\circ}$ & 1 \\
\hline HY92036 & ST1: Twin & Jun. 1992 & $50 \mathrm{~m} 35^{\circ}$ & 1 \\
\hline HY94035 & NH1-3 & Apr. 1994 & $100 \mathrm{~m} 30^{\circ}$ & 1 \\
\hline HY94036 & ST1: Twin & Apr. 1994 & $50 \mathrm{~m} 35^{\circ}$ & 1 \\
\hline HY95035 & NH1 & May 1995 & $50 \mathrm{~m} 30^{\circ}$ & 1 \\
\hline HY95036 & ST1: Twin & May 1995 & $50 \mathrm{~m} 35^{\circ}$ & 1 \\
\hline HY95068 & ST4 & Jun. 1995 & $100 \mathrm{~m} 25^{\circ}$ & 1 \\
\hline HY95041 & NT1 & Oct. 1995 & $125 \mathrm{~m} 70^{\circ}$ & 1 \\
\hline HY95071 & ST1-3 & Oct. 1995 & $100 \mathrm{~m} 35^{\circ}$ & 1 \\
\hline HY95069 & WIJ1-2 & Nov. 1995 & $125 \mathrm{~m} 20^{\circ}$ & 1 \\
\hline HY97081-1 & ST1-3: South & Aug. 1997 & $50 \mathrm{~m} 40^{\circ}$ & 2 \\
\hline HY97081-2 & ST1-3: Central & Sep. 1997 & $50 \mathrm{~m} 40^{\circ}$ & 2 \\
\hline HY97080 & NT1 & Sep. 1997 & $125 \mathrm{~m} 70^{\circ}$ & 2 \\
\hline HY98090 & ST1-3: North & Nov. 1998 & $50 \mathrm{~m} 40^{\circ}$ & 2 \\
\hline HY99091 & WIJ1-2 & Mar. 1999 & $125 \mathrm{~m} 20^{\circ}$ & 3 \\
\hline HY99102 & ST1: Twin & Mar. 1999 & $50 \mathrm{~m} 35^{\circ}$ & 3 \\
\hline HY00110 & ST1-3 & May 2000 & $50 \mathrm{~m} 35^{\circ}$ & 5 \\
\hline HY00116 & ST4 & May 2000 & $100 \mathrm{~m} 40^{\circ}$ & 5 \\
\hline HY01125 & ST1-3: North & Mar. 2001 & $50 \mathrm{~m} 40^{\circ}$ & 5 \\
\hline HY01126 & ST1-3: South & Mar. 2001 & $50 \mathrm{~m} 35^{\circ}$ & 5 \\
\hline HY02132 & NT1 & Mar. 2002 & $125 \mathrm{~m} 70^{\circ}$ & 5 \\
\hline HY02140 & ST1-3 & Aug. 2002 & $50 \mathrm{~m} 35^{\circ}$ & 5 \\
\hline NHI02001 & NH3 & Dec. 2002 & $100 \mathrm{~m} 30^{\circ}$ & 5 \\
\hline HY03152 & ST1-3: North & Mar. 2003 & $50 \mathrm{~m} 35^{\circ}$ & 5 \\
\hline HY02143 & WIJ1-2 & Jun. 2003 & $125 \mathrm{~m} 85^{\circ}$ & 5 \\
\hline HY03152-1 & ST1-3: South & Oct. 2003 & $50 \mathrm{~m} 50^{\circ}$ & 5 \\
\hline
\end{tabular}


Table 5.8: Overview of MBES surveys, survey track direction East of North $\theta^{(\mathrm{t})}$, and used survey systems for horizontal and vertical reference. The two-letter area codes are given in Table 5.2, and the system codes in Table 5.6.

\begin{tabular}{lllll}
\hline \hline survey code & area code & start & $\theta^{(\mathrm{t})}$ & system code \\
\hline RWS180-01 & NH2 & Aug. 1997 & $90^{\circ}$ & 2 \\
RWS179-01 & NH3 & Mar. 2000 & $90^{\circ}$ & 2 \\
RWS046-02 & ST1: Twin Feb. 2001 & $35^{\circ}$ & 4 \\
RWS181-01 & NH1 & May 2001 & $70^{\circ}$ & 4 \\
RWS182-01 & ST1: Twin May 2001 & $35^{\circ}$ & 4 \\
RWS043-02 & NH3 & May 2001 & $90^{\circ}$ & 4 \\
RWS044-02 & NH2 & May 2001 & $90^{\circ}$ & 4 \\
RWS045-02 & NH1 & Aug. 2001 & $70^{\circ}$ & 4 \\
RWS283-03 & NH3 & May 2002 & $90^{\circ}$ & 4 \\
RWS287-03 & NH2 & Jul. 2002 & $90^{\circ}$ & 4 \\
RWS297-03 & ST1: Twin & Mar. 2003 & $35^{\circ}$ & 4 \\
RWS292-03 & NH1 & Apr. 2003 & $70^{\circ}$ & 4 \\
RWS235-04 & NH3 & Jul. 2003 & $90^{\circ}$ & 4 \\
RWS238-04 & NH2 & Aug. 2003 & $90^{\circ}$ & 4 \\
RWS011-05 & NH1 & Apr. 2004 & $70^{\circ}$ & 4 \\
RWS012-05 & ST1: Twin & Apr. 2004 & $35^{\circ}$ & 4 \\
RWS008-05 & NH2 & May 2004 & $90^{\circ}$ & 4 \\
RWS005-05 & NH3 & Jun. 2004 & $90^{\circ}$ & 4 \\
RWS265-05 & ST1: Twin & Mar. 2005 & $35^{\circ}$ & 4 \\
HY05166 & WIJ1-2 & May 2005 & $75^{\circ}$ & 4 \\
RWS262-05 & NH2 & May 2005 & $55^{\circ}$ & 4 \\
RWS264-05 & NH1 & May 2005 & $70^{\circ}$ & 4 \\
RWS020-07 & NH3 & May 2005 & $85^{\circ}$ & 4 \\
HY05159 & NT1 & Jul. 2005 & $70^{\circ}$ & 4 \\
RWS021-07 & WIJ1-2 & Jan. 2006 & $75^{\circ}$ & 4 \\
HY06170 & ST4 & Feb. 2006 & $40^{\circ}$ & 4 \\
RWS029-07 & NH1 & Jul. 2006 & $70^{\circ}$ & 4 \\
RWS030-07 & ST1: Twin & Oct. 2006 & $35^{\circ}$ & 4 \\
\hline \hline & & & & \\
\hline
\end{tabular}




\title{
Chapter 6
}

\section{The application of estimated sea floor dynamics to improve the resurvey policy of the Netherlands Hydrographic Service}

\begin{abstract}
Bathymetric resurveying at sea is a costly process with limited resources, yet necessary for adequate nautical charts and therefore crucial for safe navigation. An important factor in an efficient resurvey policy is the type and size of sea floor dynamics. By formulating four indicators, we make recommendations for the resurvey policy of the Hydrographic Service of the Royal Netherlands Navy on the Belgian and Netherlands Continental Shelf. The continental shelf of these two countries is characterized by a sandy sea floor covered with rhythmic patterns and by limited depth. These indicators follow from the estimates for sea floor dynamics, as given by applying the statistical method of deformation analysis. We present a concept for the shallowest likely depth surface, on which we base two of the indicators. The other two indicators act as a warning: they quantify the potentially missed dynamics, which makes the procedure more robust in case of complicated morphology. We show clear differences in recommended resurvey priority between the five analyzed regions, which currently have equal resurvey frequencies.
\end{abstract}

\subsection{Introduction}

The Hydrographic Service of the Royal Netherlands Navy (RNLN) is the Dutch government office responsible for nautical surveying and charting, in order to ensure the safety of navigation at sea. To guarantee the presence of accurate information on e.g. depth on board, the usage of official nautical charts is mandatory for many types of ships. Nautical charts are based on bathymetric surveys at sea, which is costly information that expires after limited time, because of the changing nature of the sea floor in many sandy shallow seas. Especially tidal sand waves, which are rhythmic patterns that are widely present on the Belgian and Netherlands Continental Shelf (BNLCS), show dynamics for large parts of the Southern North Sea. Tidal sand waves are characterized by wavelengths of hundreds of meters and amplitudes of up to several meters. 
In order to manage bathymetric resurveys efficiently, it is necessary to plan the deployment of the two hydrographic survey vessels of the Royal Netherlands Navy according to a carefully designed resurvey policy. The policy assigns a resurvey frequency to all areas of the BNLCS under RNLN responsibility. The current policy is given in Figure 6.1. Due to various circumstances, the RNLN has not achieved these resurvey frequencies over the past years, which makes the justification of those frequencies by a comprehensive risk assessment even more relevant [Dienst der Hydrografie, 2007].

Hydrographic offices around the world are considering ways to specify resurvey frequencies for shipping routes in shallow waters. Survey policies are made worldwide, and published on the internet [NOAA Office of Coast Survey, 2008] or in the literature [De Oliveira et al., 2007; Dehling, 2006; Whatrup et al., 2005]. Ideally, the resurvey frequencies of a policy are based on five factors:

1. minimum depth;

2. draught, i.e. the depth of a ship's keel under sea level;

3. shipping intensity;

4. human interventions;

5. sea floor dynamics.

Areas that are deeper than necessary for any surface navigation do not need to be monitored as often as shallower areas (factor 1). The distinction between shallow and deep usually lies at $40 \mathrm{~m}$ [International Hydrographic Organization, 2008a]. Draught (factor 2) often is most critical in shipping lanes, which are potentially maintained at a larger depth than the surrounding sea floor.

Shipping intensity (factor 3) is important because of its direct impact on the grounding risk in case of sea floor changes, and because it is a cause of changes in depth in itself. Intense shipping increases the risk of cargo loss (like containers), which enlarges the probability of undetected objects on the sea floor. Human intervention in the natural processes of shallow sandy seas (factor 4) include dredging, sand mining and dumping, land reclamation, and the placement of objects on the sea floor (like wind farms) [Van der Veen, 2008; Roos et al., 2008]. Human intervention in natural processes also have an indirect effect that may cause long-term changes in depth until tens of kilometres away from the location of the intervention.

Present morphodynamic models allow for the qualitative prediction of the effect of human intervention in the natural morphological processes, like areas where accretion or removal of sediment is expected [Van der Veen, 2008; Roos et al., 2008], or sand wave growth and migration [Besio et al., 2008; Németh et al., 2007]. This knowledge gives opportunities to adapt resurvey frequencies based on human interventions, past or planned, in combination with observed sea floor dynamics (factor 5). This study presents a systematic way to use knowledge of sea floor dynamics to optimize the resurvey policy. Although 


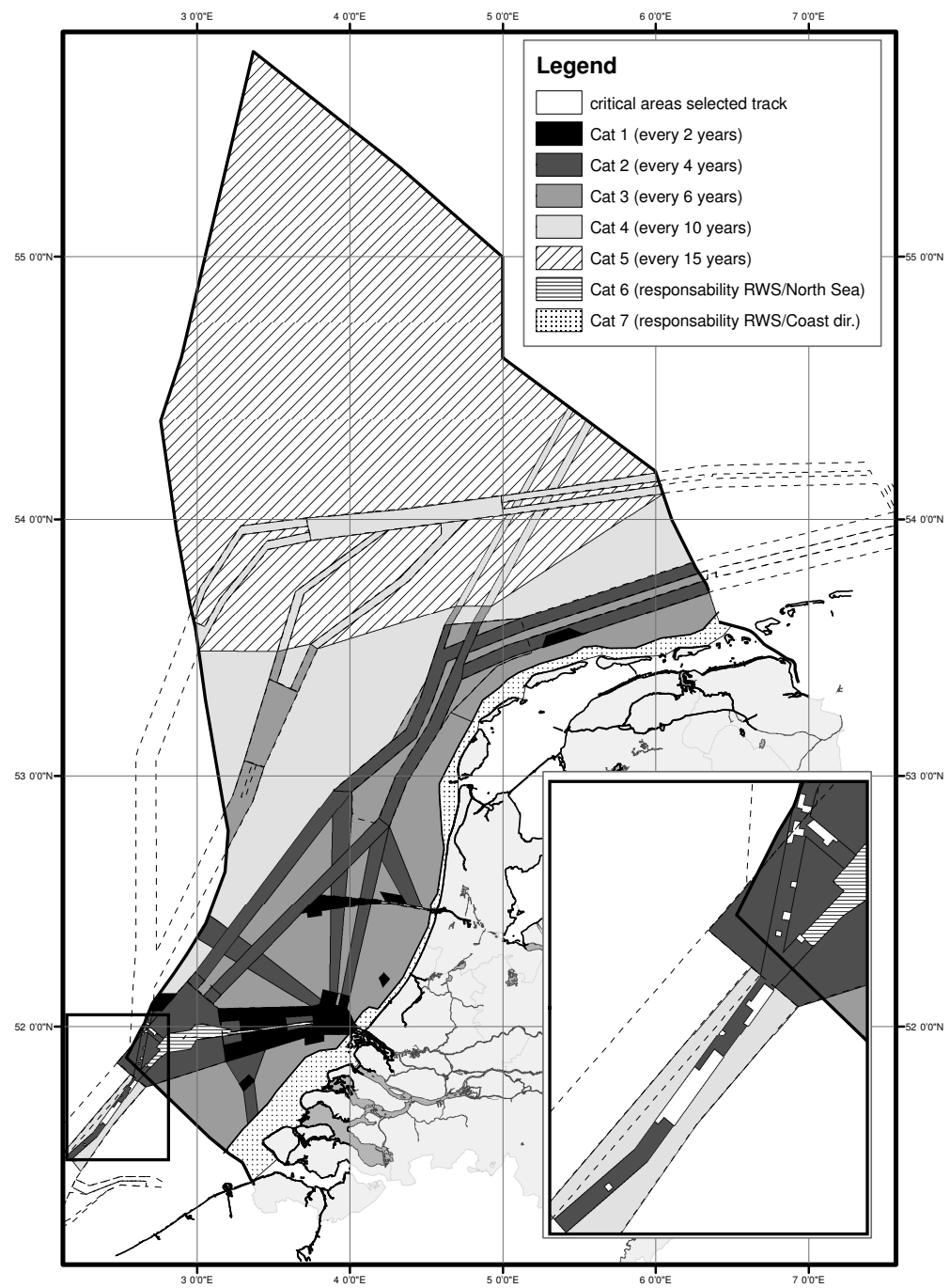

Figure 6.1: The 2003 resurvey policy of the Hydrographic Service of the Royal Netherlands Navy, for the areas of the Belgian and Netherlands Continental Shelf (BNLCS) under its responsibility. In the Selected Track for deep draught vessels, Critical areas are defined that have a resurvey frequency of once every two years. The Hydrographic Service does not survey the areas that are under responsibility of Rijkswaterstaat North Sea, or their coastal directorates. (Figure courtesy of Lt I.J. Nijman) 
several strategies currently are in use, a newly developed method known as deformation analysis (Chapter 3) gives new opportunities.

Starting with Langeraar [1966], attempts have been made to quantify dynamics of the Southern North Sea for this purpose. The most recent estimates are given in Chapter 4 and Chapter 5, using deformation analysis (Chapter 3). This method is based on statistical testing theory, and is able to estimate dynamics of the sea floor using just a few morphological parameters and their uncertainties. These estimates include the behaviour of tidal sand waves. However, the estimates of deformation analysis are not directly useful to recommend changes in resurvey frequency. Therefore, we formulate a set of indicators that enables prioritization of areas with respect to each other, for sea floor dynamics. This serves as input for a future scientific validation of the resurvey policy [Deltares, 2008].

Because of the differences in present and past resurvey frequency between the areas, the number of available surveys and the time interval of the analyses varies. Also, the morphology varies between the areas. This complicates the comparison of detected depth reductions between the areas. To remove these complications, we use observed shoaling rates, instead of observed depth differences between surveys. We reduce the resulting morphological parameters and their uncertainty to just two indicators for detected behaviour of an area.

We add another two indicators for the risk of missed dynamics to the procedure, which is necessary because some dynamics are potentially too small to be detected, given the uncertainty of the results. This is especially important because the uncertainty of areas that are showing more irregular sand wave patterns will in general be higher than the uncertainty of areas for which sand wave patterns are absent or less irregular.

First, current practice on relevant aspects of hydrography, e.g. strategies to include sea floor dynamics in resurvey policies, are given in Section 6.2. Then, four indicators for the interpretation of sea floor dynamics are introduced in Section 6.3. Indicator values for the results of the analyzed areas in the Southern North Sea are presented in Section 6.4. In Section 6.6, we discuss our findings in relation to other hydrographic and morphological concepts, and make recommendations for the resurvey policy of the Netherlands Hydrographic Service, using current hydrographic practice and the indicator results in our discussion. We finish with some conclusions in Section 6.7.

\subsection{Background: Hydrographic practice}

\subsubsection{Manual and automatic shoal biasing}

Traditionally, depth values used for nautical publications are made shallower, and thereby safer, using manual shoal biasing. Only the shallowest depth values are depicted, assuming they are representative for the shallowest value that could be present at any nearby location. Also, the isobaths, which are contour lines of equal depth, are generalized (i.e. cartographically smoothed), dependent 
on the scale, towards the shallow direction only. In this process, the experience of the cartographer plays a central role.

Recently, an alternative approach to shoal biasing was proposed, known as the navigation surface [Smith, 2003; Smith et al., 2002]. The approach of the navigation surface is algorithmic, which enables automatic processing, and thus decreases the processing time of very large MBES data sets. Also, this approach removes subjectivity from the processing of bathymetric surveys. The procedure uses depth values and their uncertainty at a grid of nodes, generated by the Combined Uncertainty and Bathymetry Estimator (CUBE) [Calder, 2003], or potentially by Kriging [e.g. Dorst and Roos, 2008; Calder, 2006].

In the navigation surface approach, isobaths are generated by defocusing using a double buffering algorithm, which generates a line at a constant distance in the deeper direction, and subsequently generates the generalized line at the same distance in the opposite direction. The constant defocusing distance is scale-dependent, allowing for various levels of generalization.

\subsubsection{Inadequately surveyed areas}

The major part of the marine environment has never been adequately surveyed [Monahan, 2007]. Even major international shipping routes have not been surveyed according to modern standards [International Hydrographic Organization, 2008b]. Therefore, only the resources that are necessary to resurvey previously surveyed areas should be spent, making more resources available to survey other areas.

To mitigate the influence of inadequately surveyed areas, various methods have been developed to indicate the quality of the survey to the mariner [Heap, 2007]. The most recent development is the introduction of zones of confidence (ZOC) of the nautical chart, especially feasible for electronic nautical charts (ENC-s) [International Hydrographic Organization, 2007; Johnson, 2004]. However, most of these concepts only focus on the quality and density of the measurements. The aging aspect is not included, as it is impossible to get reliable insight into sea floor changes, without a thorough analysis of a series of previous surveys, or the application of a morphological model. With the introduction of new analysis techniques, like the deformation analysis presented in this study, and with the ongoing development of morphological models, the inclusion of the effect of age in a quality indicator becomes possible.

\subsubsection{The inclusion of sea floor dynamics in resurvey policies}

Among hydrographic services, the following strategies are in use to deal with the factor sea floor dynamics in resurvey policies:

1. interpretation of a series of archived bathymetric surveys, as in this study;

2. use of exploratory surveys, in which a small part of an area is measured, and the decision on the resurvey of the whole area is based on the analysis of the exploratory survey; 
3. observation of the general morphology of the sea floor, using a remote sensing technique;

4. application of morphodynamic models to predict sea floor change.

The interpretation of a series of bathymetric surveys (strategy 1 ) is the most traditional method, documented by e.g. Burton [1977] and Kember [1984]. An example of the application of exploratory surveys (strategy 2) is the measurement of a single survey track over a sand dune [Le Bot et al., 2000].

An example of a remote sensing technique (strategy 3 ) is the interpretation of radar reflections on surface waves, which are influenced by changes in depth. Radar observations are available from satellites or from terrestrial stations [Calkoen et al., 1998; Vogelzang, 1997; Vogelzang et al., 1997]. Also, water color is an indicator of depth for seas with clear water [Su et al., 2008; Lafon et al., 2004]. Experiments with radar remote sensing for the North Sea have given results of mixed quality [Swart et al., 2006]. Kember [1984] stresses that any review of resurvey frequency should first investigate sea floor changes, preferably quantitatively, and subsequently should attempt to understand the morphological mechanisms (strategy 4) that cause these changes.

\subsection{Method: indicators of sea floor dynamics}

\subsubsection{Overview of deformation analysis}

\section{Input and output}

Deformation analysis is an application of statistical testing theory [e.g. Koch, 1999] to analyze a series of $S$ bathymetric surveys. The application to sea floor data requires depth values at the $P$ nodes of a grid, the uncertainty of each depth value, and the time of each survey. The size of the grid is limited to about $20 \times 20$ nodes, for computational reasons. The uncertainty is described by an error model, giving variances and covariance functions for the surveys, see Chapter 4 for the Royal Netherlands Navy (RNLN) SBES survey suite. Further, it is assumed that the performance of the Rijkswaterstaat (RWS) and RNLN MBES survey suites is according to order 1 of the S44 standard [International Hydrographic Organization, 2008a].

The procedure allows us to select $N$ significant morphological and morphodynamic parameters for an area, based on $M$ depth values $(M=P S)$, and subsequently provide estimates for these parameters, including confidence intervals. This results in a representation of the measured sea floor that consists of the characterization by a limited number of parameters, and the dispersion of the residual variations from the characterization, which is described by the variances and covariances of the parameters. The relation between the depth values and the estimated parameters are linear or linearized. This allows us to follow a Best Linear Unbiased Estimation (BLUE) for the estimation of the parameters of the characterization, and for the estimation of their dispersion (Chapter 3). 


\section{Morphological characterization}

Let us assume the input is available in a three-dimensional coordinate frame $(x, y, z)$, in which the three directions are perpendicular to each other, and depth $d$ is given as $z=-d$ at horizontal locations $(x, y)$. Deformation analysis subsequently tests extensions of the set of morphological parameters, starting with the simplest characterization possible, which is the single parameter bed level $d^{(\mathrm{b})}$, assumed constant in space and time. Then, spatial and temporal extensions to this set are made. Such an extension is accepted if the so-called test quotient $\underline{q}$ is the largest over all available extensions, and if this test quotient is larger than one. The characterization is extended until the remaining test quotients are all smaller than one.

Spatial extensions (step 1) to the initial parameter bed level $d^{(\mathrm{b})}$ are two bed slope parameters $\psi^{(\mathrm{x})}$ and $\psi^{(\mathrm{y})}$ in both the horizontal directions, and two sand wave parameters: amplitude $A$ and crest position $\xi$. Sand wave patterns are assumed to have a constant horizontal direction of the pattern variation, which is the $x$-direction of the grid, perpendicular to the constant direction of alongcrest uniformity, which is the $y$-direction of the grid. We usually work with relative sand wave parameters, with respect to their values $A_{\text {ref }}$ and $\xi_{\text {ref }}$ for a reference survey: amplitude factor $A^{\prime}=A / A_{\text {ref }}$ and migration $\xi^{\prime}=\xi-\xi_{\text {ref }}$. To determine the dominant wavelength $L^{(\mathrm{d})}$ of the sand wave extension, the test quotient becomes a function of wavelength: $q=q(L)$. Consequently, a wavelength search interval $\left[L_{\min }, L_{\max }\right]$ needs to be defined. Subsequently, the best fitting wavelength is obtained by maximizing $q(L)$. The wavelength search interval varies per area for which deformation analysis is applied (Chapter 5 ).

The dynamic extensions (step 2) define a linear parameter trend in all surveys (denoted e.g. $\dot{d}^{(\mathrm{b})}$ for bed level), or outlying parameters for survey $s$, for each $s=1, \cdots, S$ (denoted e.g. $\Delta d_{s}^{(\mathrm{b})}$ for bed level). Because the two parameters $\psi^{(\mathrm{x})}$ and $\psi^{(\mathrm{y})}$, and the two parameters $A$ and $\xi$ are mutually correlated, an extension contains the two bed slope parameters, or the two sand wave parameters. First, these two trend extensions are tested. Depending on the values of the test quotients, the trend extensions are added to the characterization. After that the $2 S$ outlier extensions are tested, to account for nonlinear behaviour or systematic errors in a survey.

The levels of significance $\alpha$ specify the probability that an extension to a representation is incorrectly selected. We use the values for $\alpha$ that were set in Chapter 4 , which are $5 \%, 7 \%$ and $5 \%$ for the spatial extensions, the trend extensions and the outlier extensions, respectively. Deformation analysis is possible using three different numbers of dimensions: a zero-dimensional analysis per grid node, a one-dimensional analysis per grid line in the direction of highest variability, and a two-dimensional analysis of a full grid. The specified levels of significance are adopted for all three numbers of dimensions. 


\section{Morphological residuals}

In our procedure, the iterative acceptance of extensions continues until all remaining extensions have a test quotient smaller than one. If the characterization fits the series of surveys well enough, from a statistical point of view, the final parameter values of that step are calculated, as well as the associated uncertainties. The overall test is used for this decision, with its overall test quotient $\underline{q}^{(\mathrm{o})}$ smaller than one to pass the test.

However, if the differences between the final characterization of a step and the measured sea floor are larger than can be explained by the uncertainty of the depth measurements, the characterization is insufficient. The differences are called the residuals, which contain measurement errors and residual variations, like asymmetries and bifurcations in the sand wave pattern, and small-scale* rhythmic features like mega-ripples. Instead of defining additional parameters, which would complicate the characterization, we assume that the residual variation is a random function, and we include additional morphological variance and covariance components in the uncertainty, using Least Squares Variant Component Estimation (LSVCE) [Teunissen and Amiri-Simkooei, 2008].

The LSVCE-procedure is followed for both the steps explained above. (To distinguish the two overall test quotients, the quotient that tests the morphological characterization in step 1 is denoted $\underline{q}^{(0, \mathrm{~m})}$, and the quotient that tests the temporal characterization in step 2 is denoted $q^{(0, t)}$.) The variances and covariances, describing the dispersion, that are calculated in step 1 are used in step 2. An increased dispersion during step 1 therefore has consequences in step 2. Firstly, it is less likely that an extension will be accepted in the second step. Secondly, the resulting dynamic parameters will be estimated less accurately. At the end of step 2, the second LSVCE procedure only influences the uncertainty of the final parameter estimates.

\subsubsection{Domains and dimensions}

One way to describe the representation is in the parameter domain, given by the $N \times 1$ vector of estimated morphological parameters $\underline{\hat{\mathbf{u}}}$ and its covariance matrix $\underline{\mathbf{C}}_{u}$, for the characterization and the dispersion respectively. The main diagonal of $\underline{\mathbf{C}}_{u}$ contains the variances $\sigma_{u}^{2}$ of the parameters $\underline{\hat{u}}$. We assume a Gaussian distribution for the deviations described by the dispersion. In vector $\underline{\hat{\mathbf{u}}}$, there are $U$ parameters $\underline{\hat{u}}_{\text {ref }}$ at reference time $t_{\text {ref }}$. Depending on the complexity of the detected dynamics, $V$ dynamic parameters $\underline{\hat{v}}$ follow the reference parameters in $\underline{\hat{\mathbf{u}}}$, with $N=U+V$ and $N \leq U S$.

Alternatively, the representation is described in the depth domain, given by the $M \times 1$ vector of estimated depth values $\underline{\hat{\mathbf{m}}}$ and and its covariance matrix $\underline{\mathbf{C}}_{m}$, at the grid nodes $\mathbf{x}_{p}$. The relation between both the domains is linear (Chapter 3):

$$
\underline{\hat{\mathbf{m}}}=\mathbf{A} \underline{\hat{\mathbf{u}}}, \quad \underline{\mathbf{C}}_{m}=\mathbf{A} \underline{\mathbf{C}}_{u} \mathbf{A}^{\mathrm{T}},
$$

* Large-scale and small-scale are used throughout this study in its general definition, meaning 'wide ranging' and 'limited ranging' respectively. In its cartographic definition, these meanings are reversed. 

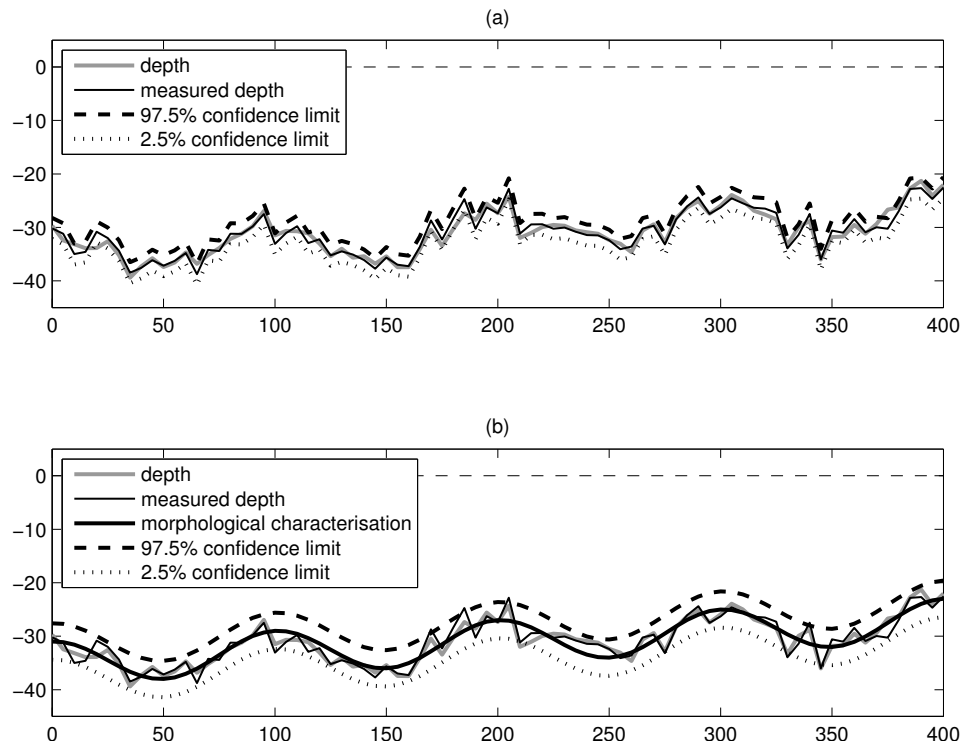

Figure 6.2: Measured depth $\underline{d}$ in relation to (a) the true, unknown depth $d$, including the $95 \%$ confidence interval around the measured depth, representing the measurement error as described by $\underline{\mathbf{C}}_{d}$; (b) morphological characterization in the depth domain $\underline{\hat{m}}$ including the $95 \%$ confidence interval around the measured depth, representing the measurement error and residual morphology as described by $\underline{\mathbf{C}}_{m}$. The difference between the measured depth and the characterization is the residual, consisting of a measurement component and usually a morphological component. The probability that the true depth $d$ is larger than the deeper limit of the confidence interval is $2.5 \%$ for both $\underline{d}_{2.5 \%}$ (graph (a)) and $\underline{\hat{m}}_{2.5 \%}$ (graph (b)), and this probability is $97.5 \%$ for the shallower limit of both $\underline{d}_{97.5 \%}$ (graph (a)) and $\underline{\hat{m}}_{97.5 \%}$ (graph (b)).

in which $\mathbf{A}$ is an $M \times N$ coefficient matrix.

The representation in the depth domain by $\underline{\hat{\mathbf{m}}}$ and $\underline{\mathbf{C}}_{m}$ is smoothed with respect to the original surveyed depth values $\underline{\mathbf{d}}$ and $\underline{\mathbf{C}}_{d}$ : the estimation procedure removes residual variations and reduces measurement errors. This is illustrated in Figure $6.2 \mathrm{a}$. The smoothing of the characterization $\underline{\mathbf{d}}$ to $\underline{\hat{\mathbf{m}}}$ is compensated for by the increase of the dispersion from $\underline{\mathbf{C}}_{d}$ to $\underline{\mathbf{C}}_{m}$, which contains a component for the residuals, as in Figure 6.2b. The variances on the main diagonal of a covariance matrix $\underline{\mathbf{C}}$ are used to construct a confidence interval around the estimates. For the $95 \%$ confidence interval, the lower limit at $2.5 \%$ is denoted by $\underline{d}_{2.5 \%}$ or $\underline{\hat{m}}_{2.5 \%}$, and the upper limit at $97.5 \%$ by $\underline{d}_{97.5 \%}$ or $\underline{\hat{m}}_{97.5 \%}$. Analogously, the estimates $\underline{d}$ and $\underline{\hat{m}}$ are denoted by $\underline{d}_{50 \%}$ and $\underline{\hat{m}}_{50 \%}$.

The more dimensions we use for the representation, the fewer the parameters in the parameter domain. A 0D approach uses the morphological parameters to describe the $P$ nodes, an 1D approach to describe $\sqrt{ } P$ grid lines (if the grid is 
square and oriented in the direction of highest variability), and a 2D approach uses only one grid. The maximum number of spatial parameters per survey per analysis $U$ is one $\left(d^{(\mathrm{b})}\right)$, four $\left(d^{(\mathrm{b})}, \psi^{(\mathrm{x})}, A^{\prime}\right.$ and $\left.\xi^{\prime}\right)$ and five $\left(d^{(\mathrm{b})}, \psi^{(\mathrm{x})}, \psi^{(\mathrm{y})}\right.$, $A^{\prime}$ and $\xi^{\prime}$ ), respectively. The maximum number of parameters is therefore the number of analyses for a grid times the maximum number of spatial parameters: $P \cdot S$ for $0 \mathrm{D}, \sqrt{ } P \cdot 4 S$ for $1 \mathrm{D}$, and $1.5 S$ for $2 \mathrm{D}$. As the $2 \mathrm{D}$ parameter vector contains fewer parameters, it is unable to represent to same sea floor complexity as the 1D vector, which causes the smoother representation in the depth domain. The 0D representation is able to use the same number of $P S$ parameters as the number of measurements, which means it is able to reproduce the original morphology at its full complexity, if the maximum number of $S$ temporal parameters would be used for all $P$ nodes.

In practice, it is rare that sand wave patterns are regular in a $2 \mathrm{D}$ analysis, see e.g. Section 6.4. This means that the $1 \mathrm{D}$ representation approximates the morphology significantly better than the $2 \mathrm{D}$ representation. Therefore, we will use the 1D results to calculate indicators for sea floor dynamics, as they give the best combination of small residuals and filtering of small-scale irregularities.

\subsubsection{Shallowest likely depth values}

\section{Prediction}

The present resurvey frequency of an area defines the expected moment of next survey $_{S+1}$ as the moment of last survey $t_{S}$ plus the resurvey period. In practice, it is not always possible to survey according to the planned resurvey frequency. Therefore, the average period between surveys $\bar{T}$ is used as the resurvey period instead:

$$
t_{S+1}=t_{S}+\bar{T}, \quad \bar{T}=\left(t_{S}-t_{1}\right) /(S-1) .
$$

To predict the expected sea floor morphology at $t=t_{S+1}$, we apply linear extrapolation. We define the $U \times 1$ vector $\underline{\hat{\underline{u}}}_{S+1}$ of predicted morphological parameters as:

$$
\underline{\hat{\mathbf{u}}}_{S+1}=\mathbf{F} \underline{\hat{\mathbf{u}}}, \quad \underline{\mathbf{C}}_{u, S+1}=\mathbf{F} \underline{\mathbf{C}}_{u} \mathbf{F}^{\mathrm{T}} .
$$

The $U \times N$ coefficient matrix $\mathbf{F}$ consists of an $U \times U$ identity matrix and an $U \times V$ submatrix for the dynamic parameters in $\underline{\hat{\mathbf{u}}}$. The columns for the outlier estimates $\Delta \underline{u}_{s}$ are vectors of zeros, and the columns for the trend estimates $\underline{\dot{u}}$ correspond to the vector of time differences $t_{s}-t_{\text {ref }}$. This means that outliers in the parameters are ignored for the predictions, and trends are linearly extrapolated.

As an example, it follows from equation (6.3) for the prediction of a parameter $u$ that:

$$
\underline{\hat{u}}_{S+1}= \begin{cases}\hat{\underline{u}}_{\mathrm{ref}} & (\text { if } u \text { static }) \\ \underline{\hat{u}}_{\mathrm{ref}}+\left(t_{S+1}-t_{\mathrm{ref}}\right) \underline{\hat{\dot{u}}} & (\text { if } u \text { dynamic }) .\end{cases}
$$


Correspondingly, the variance of the predicted parameter $\underline{\hat{u}}_{S+1}$ is:

$$
\underline{\sigma}_{u, S+1}^{2}= \begin{cases}\underline{\sigma}_{u, \text { ref }}^{2} & \text { (if } u \text { static }) \\ \underline{\sigma}_{u, \text { ref }}^{2}+\left(t_{S+1}-t_{\mathrm{ref}}\right)^{2} \underline{\sigma}_{\dot{u}}^{2} & (\text { if } u \text { dynamic }) .\end{cases}
$$

If no trend has been detected for the parameter $u$, the estimates $\underline{\hat{u}}_{S+1}$ and their variances $\sigma_{u, S+1}^{2}$ are static, i.e. independent of the moment of prediction $t_{S+1}$. If the value of a parameter contains a trend, the variances $\sigma_{u, S+1}^{2}$ increase in time.

The $P \times U$ coefficient matrix $\mathbf{A}_{S+1}$ describes the relation between the predicted representations in the parameter domain and the depth domain. The elements of $\mathbf{A}_{S+1}$ correspond to the elements in the first $U$ columns of $\mathbf{A}$. The predicted representation in the depth domain follows from the predicted representation in the parameter domain according to:

$$
\underline{\hat{\mathbf{m}}}_{S+1}=\mathbf{A}_{S+1} \underline{\hat{\mathbf{u}}}_{S+1}, \quad \underline{\mathbf{C}}_{m, S+1}=\mathbf{A}_{S+1} \underline{\mathbf{C}}_{u, S+1} \mathbf{A}_{S+1}^{\mathrm{T}} \text {. }
$$

The extended representation in the depth domain contains the representations for all the surveys $s$, and the predicted representation. It is denoted $\underline{\hat{\mathbf{m}}}^{+}$and $\underline{\mathbf{C}}_{m}^{+}$ for its $P(S+1) \times 1$ characterization vector and $P(S+1) \times P(S+1)$ covariance matrix.

\section{Depth reduction}

The crucial property of a bathymetric representation for maritime navigation is the shallowest depth that is expected. Depth is already reduced for the water level above the reference low-water surface, during the processing of the survey. We reduce it further to create a safe margin for navigation in areas that are potentially dynamic. For this reduction, we use the extended representation in the depth domain. We assume a Gaussian distribution for the deviations, as described by the dispersion, and specify a $97.5 \%$ confidence level to the requirement that the depth should not be shallower than given by the representation.

This enables us to compute a depth-reduced characterization:

$$
\underline{\hat{\mathbf{m}}}_{97.5 \%}^{+}=\underline{\hat{\mathbf{m}}}^{+}-1.96 \sqrt{\operatorname{diag}\left(\underline{\mathbf{C}}_{m}^{+}\right)}
$$

The operator $\operatorname{diag}()$ converts the main diagonal of the covariance matrix into a column vector of variances. We regard it unlikely that the true depth $d$ is shallower than its element in $\underline{\hat{\mathbf{m}}}_{97.5 \%}^{+}$, as depicted by the upper confidence limit in Figure $6.2 \mathrm{~b}$. Therefore, the vector $\underline{\hat{\mathbf{m}}}_{97.5 \%}^{+}$contains the shallowest likely depth values. It represents the $S+1$ shallowest likely depth surfaces $\underline{\hat{m}}_{97.5 \%}(x, y)$. 


\subsubsection{Two indicators for rates of change}

\section{The creation of a bias in the shallow direction}

For a static sea floor, the estimated depth values and uncertainties are constant. Consequently, the shallowest likely depth values are constant, and therefore their rates of change are zero.

For a dynamic sea floor, the predicted depth values $\underline{\underline{\hat{\mathbf{m}}}}_{S+1}$ are likely to have the largest uncertainty. This means for the rates that the addition of the prediction creates a bias towards the shallow direction, shown in Figure 6.3. The less frequent a resurvey is done, the larger the time span of the prediction becomes. Large predictive time spans relative to the size of a trend result in a larger positive bias of the shoaling rate. This makes a rate a valuable indicator for the necessity to change the resurvey frequency of an area, in comparison to other areas.

\section{The shallowest likely depth rate}

For a mariner, it is not important to know where exactly the shallowest likely depth is located. Instead, it is important to know what the overall shallowest likely depth is of the whole area, and how this depth evolves in time. Therefore, we select the minimum over the $P$ depth values in subvector $\underline{\hat{\mathbf{m}}}_{97.5 \%, s}^{+}$per survey $s$.

We use these $S+1$ overall shallowest likely depth values for a least-squares estimation of their rate of change in a linear regression analysis, using the variances of the corresponding depth values $\underline{\hat{\underline{m}}}_{p, s}^{+}$as weights. The result is the shallowest likely depth rate (SLDR), depicted in Figure 6.4. Because the variances of the depth values are used for the estimation of the SLDR, a variance is associated with it, describing its uncertainty. Also, we calculate a correlation coefficient $\rho^{(\mathrm{S})}$ to express the deviations of the shallowest likely depth values from the estimated linear rate.

\section{The maximum estimated shoaling rate}

To decide whether a resurvey is necessary, the shallowest likely depth in an area alone is not sufficient. In addition, the shoaling rate at any point $p$ within the area is necessary information. The decision if a shallower depth is relevant does not only depend on its position on top of or away from a sand wave crest, but also on the chart scale. The SLDR does not detect a migration of the shallowest likely depth, which is important information for the update of detailed charts. Therefore, we estimate a rate of change at each grid node, using the $S+1$ depth values in subvector $\underline{\hat{\mathbf{m}}}_{97.5 \%, p}^{+}$.

Again, we perform least-squares estimations of the rates of change, by calculating a linear regression per grid node with the variances as weights. We regard it unlikely that the true shoaling rate at any position within the area is larger than the maximum estimated shoaling rate (MESR) of the shallowest 
(a)

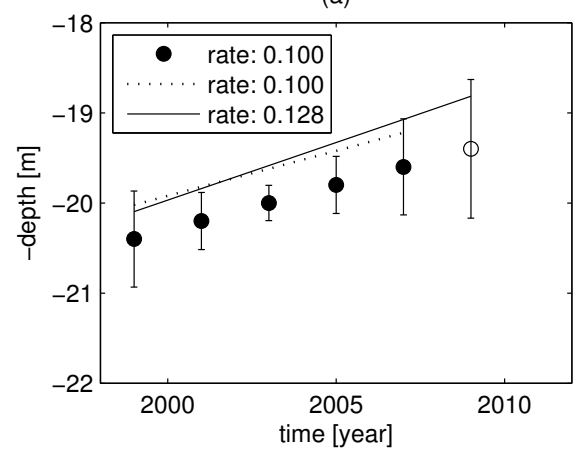

(c)

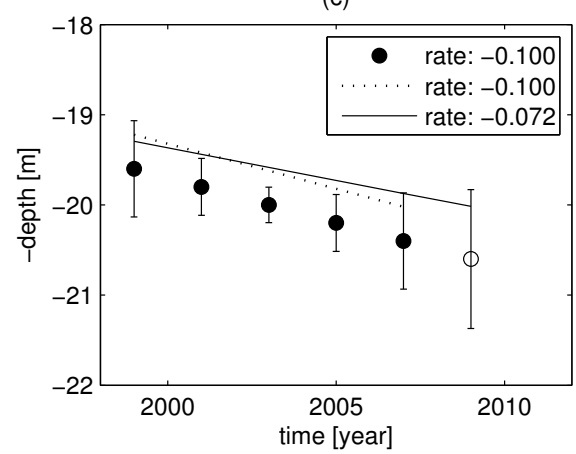

(b)

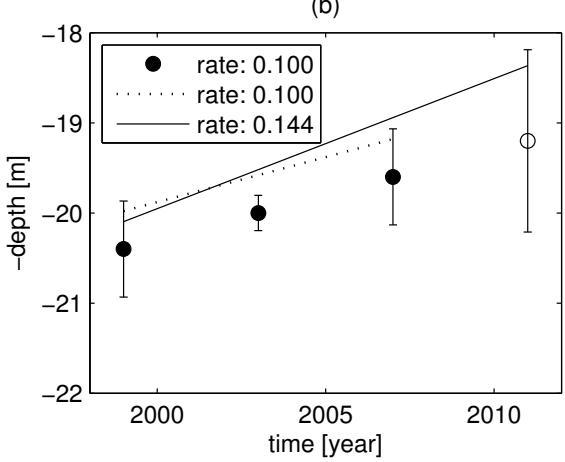

(d)

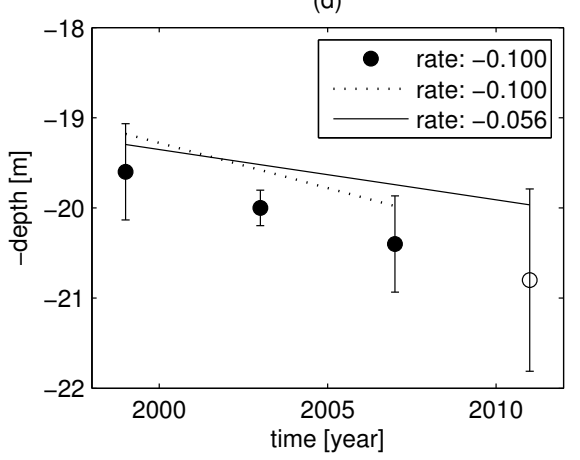

Figure 6.3: Creation of a bias in a rate of change of the shallowest likely depth, by adding a prediction (white circle) to the survey moments (black circles): (a) depth decrease, two year resurvey frequency; (b) depth decrease, four year resurvey frequency; (c) depth increase, two year resurvey frequency; (d) depth increase, four year resurvey frequency.

likely depth values for the $97.5 \%$ confidence level at the grid nodes $p=1, \cdots, P$. The MESR is also depicted in Figure 6.4. Because the variances of the depth values are used for the estimation of the MESR, a variance is associated with it, describing its uncertainty. Also, we calculate a correlation coefficient $\rho^{(\mathrm{M})}$ to express the deviations of the shallowest likely depth values from the estimated linear rate.

\subsubsection{Two indicators for the risk of missed dynamics}

\section{Introduction}

Deformation analysis is able to detect smaller dynamics for less irregular sand waves, as those sand waves are represented better by the estimated parameters. 


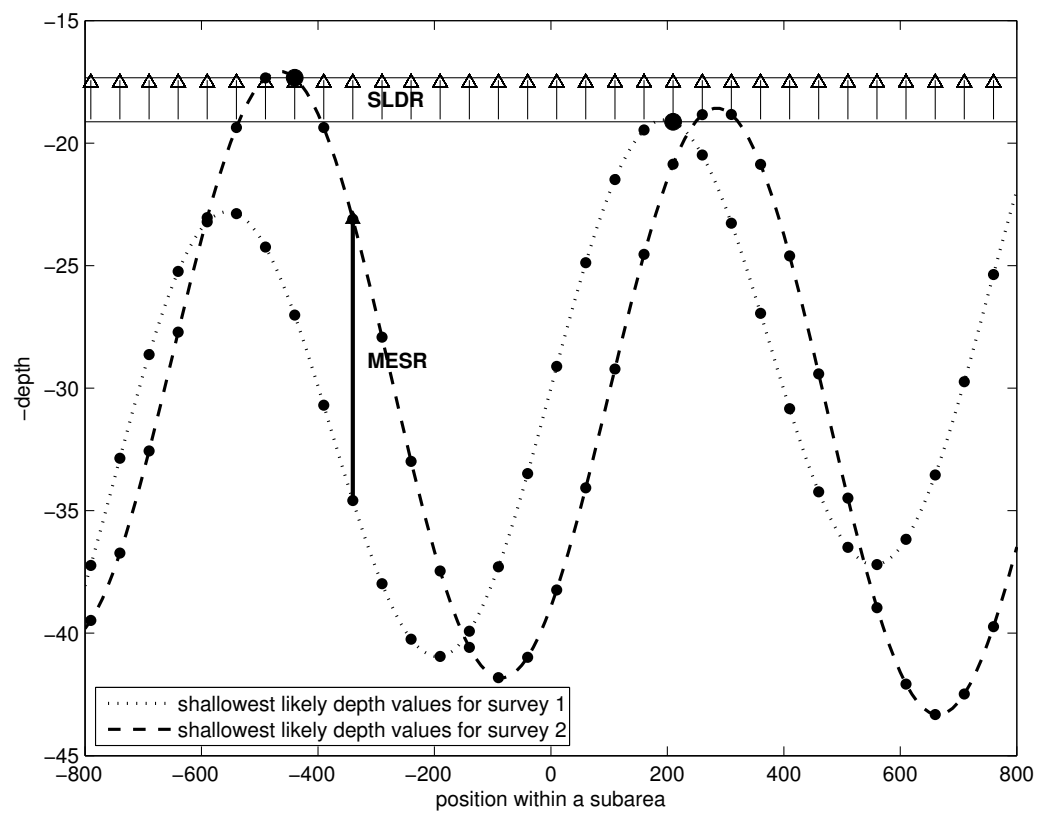

Figure 6.4: The calculation of the SLDR and MESR from the shallowest likely depth values of two surveys, measured a year apart. The SLDR is based on the overall shallowest likely depth values of each survey, indicated by the larger black circles, while the MESR is the maximum of the rates of the shallowest likely depth values at the grid nodes.

In that case, the residuals are smaller, resulting in smaller detectable spatial and dynamic parameters. It also results in smaller variations between the parameter estimates and in parameter existence between the areas. A quantification of the irregularity of sand waves would therefore be a valuable predictor for the performance of our implementation of deformation analysis. In the determination of sand wave irregularity, it is helpful to distinguish between irregularities that are caused by the shape of the pattern in $x$-direction and irregularities caused by the continuation of the pattern in $y$-direction. We term the first type wave irregularity and the second type crest irregularity. We define one-dimensional sand wave irregularity as the difference with the estimated sinusoidal wave in the direction across the crest. This difference is given by a variable for the size of the morphological residuals, as explained in more detail below. Twodimensional irregularity also contains the variation of the sand wave pattern in the direction along the crest.

Two types of wave irregularity are distinguished: horizontal wave asymmetry (also lee-stoss asymmetry and skewness) and vertical wave asymmetry (also crest-trough asymmetry and peakedness) of the sand wave pattern. Horizon- 
(a) symmetrical sand wave

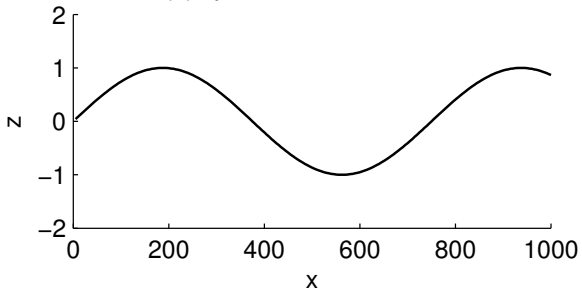

(c) wave irregularity: horizontal asymmetry

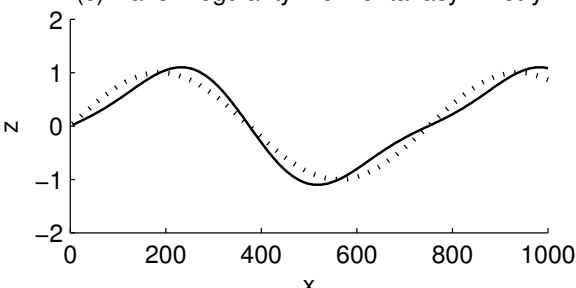

(e) wave irregularity: vertical asymmetry

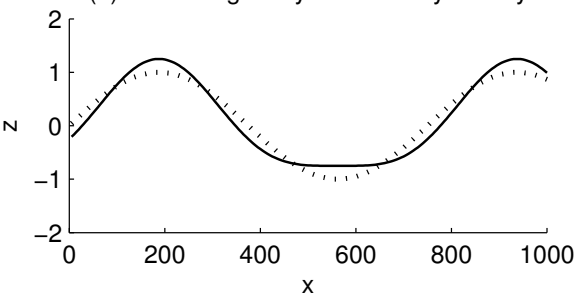

(b) infinite crest length

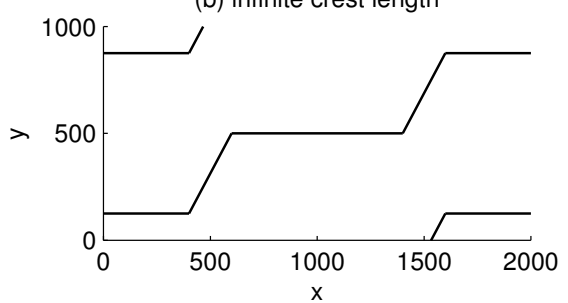

(d) crest irregularity: finite crest length

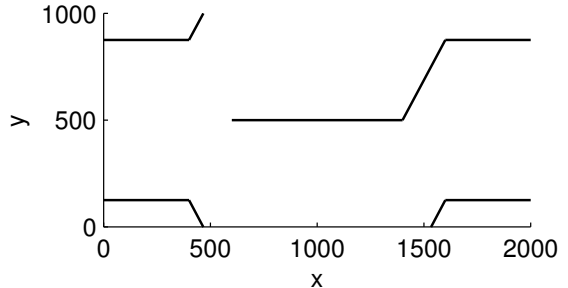

(f) crest irregularity: bifurcation

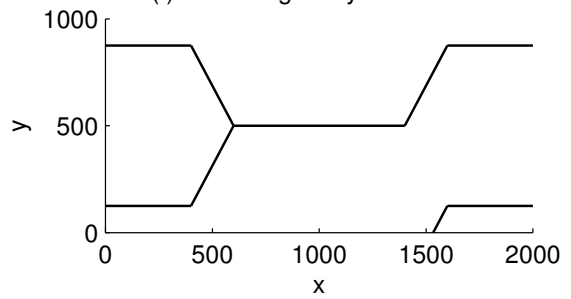

Figure 6.5: Wave irregularity shown by depth values in an $(x, z)$ coordinate frame (left) and crest irregularity shown by crest positions in an $(x, y)$ coordinate frame (right). The graphs on the left show: (a) wave symmetry; (c) horizontal asymmetry (skewness); and (e) vertical asymmetry (peakedness) of the crests. The graphs on the right show: (b) shifting crest segments (d) finite-length crests; and (f) bifurcations. In the graphs (c) and (e), the original symmetrical sand wave is shown dotted.

tal asymmetry is explained by residual currents or by tidal asymmetry [Besio et al., 2008; Németh et al., 2007], and indicates migration of the pattern [Knaapen, 2005]. Crest irregularities include shifting crest segments, bifurcations and finite-length crests. They could be explained by differences in migration rate between parts of the pattern, e.g. as a result of differences in depth (Chapter 4). Examples of these irregularities are given in Figure 6.5.

A first distinction between regular and irregular sand waves is made using the overall test quotient. To enable a comparison between areas, we subsequently quantify the degree of irregularity, for which we use the morphological variance. Finally, the effect of irregularity on the minimal detectable dynamics is illustrated. The minimal detectable dynamics are necessary to interpret the results, used to adapt the resurvey policy. 


\section{Regular versus irregular sand wave patterns}

The sinusoidal sand wave extension of step 1 in the deformation analysis procedure (Chapter 3) represents a perfectly regular sand wave. This step selects the required spatial parameters by adding extensions to the morphological characterization. The differences between the characterization and the measurements are the estimated residuals, in vector $\underline{\hat{\mathbf{r}}}$. In this first step, the overall test quotient $\underline{q}^{(\mathrm{o}, \mathrm{m})}$ represents the size of the square of the estimated residuals in relation to the measurement and interpolation uncertainty, which is described by covariance matrix $\mathbf{C}_{d}$.

For the estimation of $N$ morphological parameters from $M$ measured depth values, the overall test quotient $\underline{q}^{(\mathrm{o})}$ is given as (Chapter 3):

$$
\underline{q}^{(\circ)}=\underline{\hat{\mathbf{r}}}^{\mathrm{T}} \mathbf{C}_{d}^{-1} \underline{\hat{\mathbf{r}}} /(M-N) .
$$

The division by the so-called redundancy $M-N$ averages the estimated residuals. Therefore, the overall test quotient expresses how much of the differences between the measured pattern and the best approximation by a sinusoidal wave could be due to the measurement and interpolation error $\underline{e}$, and how much of that difference remains to be explained as sand wave irregularity.

An area for which the overall test quotient $q^{(0, m)} \leq 1$ is called regular: all residual variation can be explained by the measurement and interpolation uncertainty. If $q^{(\mathrm{o}, \mathrm{m})}>1$, it is called irregular, because a part of the residual variation has to be explained by morphological deviations from the sinusoidal sand wave characterization.

\section{The degree of irregularity of a pattern}

The initial covariance matrix of step 1 only contains values for the measurement and interpolation errors, and is denoted $\mathbf{C}_{d}^{(\mathrm{e})}$. After the Least-Squares Variance Component Estimation (LSVCE) [Teunissen and Amiri-Simkooei, 2008], the covariance matrix also contains morphological covariance components. The additional components are given in covariance matrix $\mathbf{C}_{d}^{(\mathrm{m})}$. For details, see Chapter 3. The covariance matrix at the end of step 1 is therefore

$$
\mathbf{C}_{d}^{(\mathrm{em})}=\mathbf{C}_{d}^{(\mathrm{e})}+\mathbf{C}_{d}^{(\mathrm{m})} .
$$

In the LSVCE procedure, we divide each of the two contributing covariance matrices into a matrix that only contains the variances $\underline{\mathbf{C}}_{d}^{(\mathrm{v})}$ on its main diagonal, and a matrix $\underline{\mathbf{C}}_{d}^{(\mathrm{c})}$ that only contains covariances, and therefore has zeros on its main diagonal. Further, the presence of small-scale morphological variations, like mega-ripples, possibly differs per survey, due to e.g. extreme meteorological events, and consequently we need to make a distinction between the morphological components per survey as well, denoted $\underline{\mathbf{C}}_{d, k}$, for $k=1, \cdots, S$. In such a matrix, the elements for all other surveys are set to zero. For computational reasons, it is attractive to combine the estimation of the covariance functions 
of the the error contribution and the morphological contribution into one covariance component per survey. The resulting expression for the addition of $K=2 S$ components is (Chapter 3 ):

$$
\underline{\mathbf{C}}_{d}^{(\mathrm{em})}=\mathbf{C}_{d}^{(\mathrm{e}, \mathrm{v})}+\sum_{k=1}^{S} \underline{\mathbf{C}}_{d, k}^{(\mathrm{m}, \mathrm{v})}+\sum_{k=1}^{S} \underline{\mathbf{C}}_{d, k}^{(\mathrm{em}, \mathrm{c})}
$$

The covariance components are estimated using the fit of a positive-definite function through empirical auto-covariance values (Chapter 2). The variance components are estimated by the estimation of variance factors $\underline{\hat{\sigma}}_{s}^{(\mathrm{m}) 2}$ from the residuals, using a least-squares procedure [Teunissen and Amiri-Simkooei, 2008]. Using a $P \times P$ identity matrix $\mathbf{I}_{P}$, we define the morphological variance component according to:

$$
\underline{\mathbf{C}}_{d, s}^{(\mathrm{m}, \mathrm{v})}=\underline{\hat{\sigma}}_{s}^{(\mathrm{m}) 2} \mathbf{I}_{P}
$$

where the $P \times P$ matrices $\underline{\mathbf{C}}_{d, s}^{(\mathrm{m}, \mathrm{v})}$ contain the non-zero elements of the $M \times M$ matrices $\underline{\mathbf{C}}_{d, k}^{(\mathrm{m}, \mathrm{v})}$ on their main diagonal. The morphological variance components resulting from the one-dimensional analyses give us the wave irregularity, and the two-dimensional morphological variance component includes the influence of both wave and crest irregularity.

\section{The effect of irregularity on the detection of dynamics}

To quantify the dynamics that can still be found in the presence of a certain irregularity, we calculate the size of dynamics that are minimal detectable, as a function of the morphological variance. Minimal Detectable Biases (MDB-s) are calculated as (Chapter 3):

$$
\left|v_{a}\right|=\sqrt{\frac{\lambda_{a}}{\mathbf{b}_{a}^{\mathrm{T}} \mathbf{C}_{d}^{-1}\left(\mathbf{C}_{d}-\mathbf{C}_{m}\right) \mathbf{C}_{d}^{-1} \mathbf{b}_{a}}} .
$$

The covariance matrix $\mathbf{C}_{m}$ of the characterization $\underline{\hat{\mathbf{m}}}$ in the depth domain was defined in equation (6.1), and relates to the covariance matrix $\mathbf{C}_{d}$ as:

$$
\mathbf{C}_{m}=\mathbf{A}\left(\mathbf{A}^{\mathrm{T}} \mathbf{C}_{d}^{-1} \mathbf{A}\right)^{-1} \mathbf{A}^{\mathrm{T}}
$$

The noncentrality parameter $\lambda_{a}$ depends on the chosen level of significance $\alpha$ of the test, the definition of the extension (via its number of degrees of freedom $Q)$, and the power $\gamma$ of the test: $\lambda=\lambda(\alpha, Q, \gamma)$. An extension is defined as $\mathbf{B}_{a} \mathbf{v}_{a}$, in which the $M \times Q$ matrix $\mathbf{B}_{a}$ contains the coefficients, and the $Q \times 1$ vector $\mathbf{v}_{a}$ the parameters of the tested extension $a$. In the case of an extension with one degree of freedom, $Q=1$, the matrix $\mathbf{B}_{a}$ reduces to an $M \times 1$ vector $\mathbf{b}_{a}$, and the vector $\mathbf{v}_{a}$ to a single parameter $v_{a}$.

Analogously to Chapter 4, we use a power $\gamma$ of $50 \%$, to obtain the dynamics that are as often detected as they are not. We also use a power of $95 \%$, to 
compare the MDB-s to depth uncertainty at a $95 \%$ confidence interval. The levels of significance for a trend and an outlier are defined in Chapter 4 as $7 \%$ and $5 \%$, respectively. The power is the probability of correctly detecting dynamics, and the level of significance is the probability of incorrectly accepting an extension for a certain type of dynamics.

We set the $M \times N$ matrix of coefficients A according to a characterization in step 1 with sloping and sand wave parameters. In step 2 this means an initial static situation of $N=5$ parameters for a two-dimensional grid analysis, and $N=4$ for a one-dimensional grid line analysis. The vector $\mathbf{b}_{a}$ is consecutively modeled for types of dynamics $a$ of a single parameter: a trend in depth, an outlier in depth for one of the surveys, a trend in amplitude factor, and an outlier in amplitude factor.

Now, let us assume that we are able to measure the sea floor with a constant variance of $\sigma^{(\mathrm{e}) 2}$, at every position we need, without correlations. In that case, the covariance matrix of measurement and interpolation errors is $\sigma^{(\mathrm{e}) 2} \mathbf{I}_{M}$. If, in addition, the morphological variance is constant over time, and the morphological residuals are spatially uncorrelated, the morphological covariance matrix is defined as $\underline{\hat{\sigma}}^{(\mathrm{m}) 2} \mathbf{I}_{M}$, as in equation (6.11). In that case, the covariance matrix $\mathbf{C}_{d}^{(\mathrm{em})}$ reduces to $\underline{\hat{\sigma}}^{(\mathrm{em}) 2} \mathbf{I}_{M}$, see equation (6.9), where we define $\underline{\hat{\sigma}}^{(\mathrm{em}) 2}$ as $\sigma^{(\mathrm{e}) 2}+\underline{\hat{\sigma}}^{(\mathrm{m}) 2}$.

As a consequence of these simplifications, we find a relation between the MDB and the estimated morphological variance, for dynamics of each of the four types $a$ :

$$
\left|\underline{v}_{a}\right|=\sqrt{\frac{\lambda_{a} \hat{\underline{\sigma}}^{(\mathrm{em}) 2}}{\mathbf{b}_{a}^{\mathrm{T}}\left(\mathbf{I}_{M}-\mathbf{A}\left(\mathbf{A}^{\mathrm{T}} \mathbf{A}\right)^{-1} \mathbf{A}^{\mathrm{T}}\right) \mathbf{b}_{a}} .}
$$

Larger dynamics, or biases, can be detected with a higher probability than $\gamma$, and smaller biases with a smaller probability. Even a very small bias can be found, with a small probability, but a very large bias will remain undetected with a certain small probability as well.

Examples of the values of the dynamics that are minimal detectable are given in Table 6.1. The coefficient matrix $\mathbf{A}$ is specified using the following details: for the one-dimensional MDB-s, a grid line of twenty nodes is defined, and for the two-dimensional MDB-s, the grid has a size of twenty times twenty nodes; the grid spacing is $50 \mathrm{~m}$; six surveys in ten year are done; a sinusoidal sand wave with a wavelength of $750 \mathrm{~m}$ is present; there are no other sea floor dynamics than the single type that is specified. The constant error variance $\sigma^{(\mathrm{e}) 2}$ is set at $0.1 \mathrm{~m}^{2}$.

Because of the absence of covariances for the MDB calculation, we should expect that these MDB-s are not detected with the specified power in reality. Nevertheless, Table 6.1 illustrates that trends are easily detectable, also for larger morphological variances and the one-dimensional grid line analysis. Table 6.1 also illustrates that outlying values are much harder to detect, in the cases of a relatively large morphological variance. Also, it is clear that it is 
Table 6.1: Values of one-dimensional and two-dimensional MDB-s for the detection of dynamics in step 2. The MDB-s are given for levels of significance $\alpha$ of $7 \%$ for trends and $5 \%$ for outliers, at a power $\gamma$ of $50 \%$ and $95 \%$, and a defined morphological variance $\sigma^{(\mathrm{m}) 2}$ of $0.1,1$, and $10 \mathrm{~m}^{2}$. It is assumed that there are uncorrelated measurement and interpolation errors with a variance $\sigma^{(\mathrm{e}) 2}$ of $0.1 \mathrm{~m}^{2}$. For the one-dimensional MDB-s, a grid line has $\sqrt{ } P=20$ nodes, and for the two-dimensional MDB-s, the grid has $P=20 \times 20$ nodes. The grid spacing is $50 \mathrm{~m}$, and $S=6$ surveys in ten year are done. (The resurvey period is $T=2$ year.) The results are valid for a sand wave with a dominant wavelength $L^{(\mathrm{d})}$ of $750 \mathrm{~m}$, and no other sea floor dynamics than specified. The MDB-s are given for a new survey, two years after the sixth.

\begin{tabular}{|c|c|c|c|c|}
\hline \multirow[t]{2}{*}{ type of dynamics } & $\overline{\sigma^{(\mathrm{m}) 2}}$ & $\mathrm{MDB}(1 \mathrm{D})$ & \multicolumn{2}{|l|}{$\mathrm{MDB}(2 \mathrm{D})$} \\
\hline & {$\left[\mathrm{m}^{2}\right]$} & $\gamma=50 \% ; 95 \%$ & $\gamma=50 \% ; 95 \%$ & \\
\hline linear trend in depth & 0.1 & $0.03 ; 0.06$ & $0.007 ; 0.013$ & $\mathrm{~m} / 2 \mathrm{yr}$ \\
\hline linear trend in depth & 1 & $0.10 ; 0.19$ & $0.02 ; 0.04$ & $\mathrm{~m} / 2 \mathrm{yr}$ \\
\hline linear trend in depth & 10 & $0.31 ; 0.59$ & $0.07 ; 0.13$ & $\mathrm{~m} / 2 \mathrm{yr}$ \\
\hline outlying depth & 0.1 & $0.21 ; 0.39$ & $0.05 ; 0.09$ & $\mathrm{~m}$ \\
\hline outlying depth & 1 & $0.50 ; 0.92$ & $0.11 ; 0.21$ & $\mathrm{~m}$ \\
\hline outlying depth & 10 & $1.53 ; 2.80$ & $0.34 ; 0.63$ & $\mathrm{~m}$ \\
\hline linear trend in amplitude & 0.1 & $0.04 ; 0.08$ & $0.009 ; 0.017$ & $\mathrm{~m} / 2 \mathrm{yr}$ \\
\hline linear trend in amplitude & 1 & $0.13 ; 0.25$ & $0.03 ; 0.06$ & $\mathrm{~m} / 2 \mathrm{yr}$ \\
\hline linear trend in amplitude & 10 & $0.40 ; 0.77$ & $0.09 ; 0.17$ & $\mathrm{~m} / 2 \mathrm{yr}$ \\
\hline outlying amplitude & 0.1 & $0.28 ; 0.52$ & $0.06 ; 0.12$ & $\mathrm{~m}$ \\
\hline outlying amplitude & 1 & $0.66 ; 1.21$ & $0.15 ; 0.27$ & $\mathrm{~m}$ \\
\hline outlying amplitude & 10 & $1.99 ; 3.66$ & $0.45 ; 0.82$ & $\mathrm{~m}$ \\
\hline
\end{tabular}

about twice as hard to detect dynamics with a power of $95 \%$ than with a power of $50 \%$.

As Table 6.1 already illustrates, the outlying sand wave amplitude is the hardest type of dynamics to detect. Because we hardly found any amplitude growth in the studied regions (Chapters 4 and 5), and migration does not affect the overall shallowest likely depth values, we instead use the MDB-s for a depth outlier as indicators.

It is also clear from Table 6.1 that more dynamics can be detected by a twodimensional grid analysis than by a one-dimensional grid line analysis, provided that the specified dynamics are constant over the grid. As shown in Chapter 5, for some subareas dynamics are found for several grid lines, while the grid analysis does not show dynamics. Because bed level dynamics are in practice not always constant over a grid, but also often do not happen in specific directions only, we use both the one-dimensional and the two-dimensional MDB-s as indicators. A power $\gamma$ of $95 \%$ is chosen, to allow for a comparison with the $95 \%$ confidence interval for depth uncertainty, as specified by S44, for the appropriate order and at the observed bed level [International Hydrographic Organization, 2008a]. 


\subsubsection{Combination of the four indicators}

In this Section, we describe how we combine the four indicators introduced previously: SLDR, MESR, 1D MDB, and 2D MDB. The two indicators SLDR and MESR work well in combination: in the calculation of the SLDR, spatial differences are eliminated first, and the rate of change for a grid is estimated next. In contrast, the calculation of the MESR first estimates rates of change at each node, and space is eliminated next. These properties cause sand wave growth to dominate the SLDR, and sand wave migration to dominate the MESR. The MDB indicators are a useful addition, because they provide an extra warning against dynamics that are potentially missed by the first two indicators.

An indicator is termed large if it exceeds a critical value. For the SLDR and the MESR, the critical values are their uncertainties at a $95 \%$ level. For the MDB-s, we use vertical uncertainties as stated by the IHO S44 standards, which are depth-dependent.

We use the following priority scheme.

1. Assign first priority to areas that have large positive SLDR-s, because the minimum depth values decrease for those areas.

2. Assign second priority to the other areas that have large positive MESR$\mathrm{s}$, because there are locations within those areas where the depth values decrease.

3. Assign third priority to the other areas that have large two-dimensional MDB-s, because large-scale dynamics of significant size could be missed in those areas.

4. Assign fourth priority to the other areas that have large one-dimensional MDB-s, because small-scale dynamics of significant size could be missed in those areas.

5. Assign fifth priority to all other areas.

\subsection{Results: indicator values for the Southern North Sea}

\subsubsection{The two rates of change}

The morphodynamics of five regions in the Southern North Sea have been estimated using deformation analysis. Results are given in Chapter 4 for an area West of Rotterdam, and in Chapter 5 for four other regions. The regions are shown in Figure 6.6, and the areas per region are listed in Table 6.2. Areas are subdivided into subareas, for each of which a grid is available.

An overview of the resulting indicators SLDR and MESR is given in Table 6.3. The SLDR values are all close to zero, meaning that the shallowest likely depth values per subarea hardly change. Exceptions are the SLDR values for the SBES surveys of the Noordhinder Junction, as it indicates that the shallowest likely depth per subarea is increasing. This confirms the results for those surveys (Chapter 5). The flat, shallow area in the TE-TSS and the three 


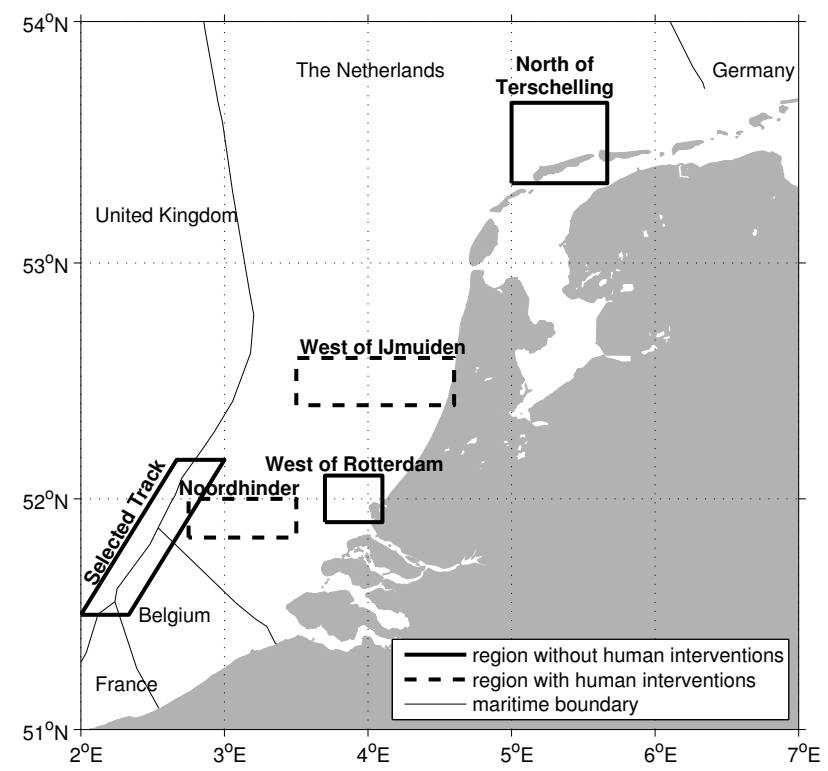

Figure 6.6: Overview of analyzed regions on the Belgian and Netherlands Continental Shelf.

Table 6.2: Areas per region, including codes for areas and regions used in the following Tables.

\begin{tabular}{ll}
\hline \hline region & areas \\
\hline Selected Track (ST) & Critical areas A, including Twin (ST1); \\
& Critical areas B, E, F, G, H, I, J (ST2); \\
& Critical areas C, D, K (ST3); \\
& Long Stay anchorage (ST4) \\
& Noordhinder Junction (NH1); \\
Noordhinder (NH) & Short Stay anchorage (NH2); \\
& Eurogeul Approach (NH3) \\
West of Rotterdam (WR) & Maas West anchorage (WR1) \\
West of IJmuiden (WIJ) & IJgeul Approach (WIJ1); \\
& IJgeul Approach anchorage (WIJ2) \\
North of Terschelling (NT) & shallow part of TE-TSS (NT1) \\
\hline \hline
\end{tabular}


Table 6.3: Averages per area for the SLDR and MESR values $[\mathrm{m} / \mathrm{yr}]$, followed by their uncertainties expressed as $95 \%$ confidence limits, and correlation coefficients $\rho$. Positive rates mean the sea floor becomes shallower. Static areas have an average rate of exactly 0 , and small rates are denoted 0.00 . The two-letter region codes are given in Table 6.2. The areas Maas west anchorage (WR1), IJgeul Approach and IJgeul Approach anchorage (WIJ1-2) have MESR values that are higher than their $95 \%$ confidence limits, and are therefore marked with (!).

\begin{tabular}{lrrrr}
\hline \hline area (\# subareas) & SLDR & $\rho^{(\mathrm{S})}$ & MESR & $\rho^{(\mathrm{M})}$ \\
\hline ST1, 1991-1999 (25) & $0 \pm 0.05$ & 1 & $0.00 \pm 0.04$ & 0.93 \\
ST1, 2000-2002 (25) & $0 \pm 0.16$ & 1 & $0 \pm 0.19$ & 1 \\
ST1, 2003-2006 (25) & $0 \pm 0.10$ & 1 & $0 \pm 0.13$ & 1 \\
ST2 (20) & $0 \pm 0.11$ & 1 & $0 \pm 0.19$ & 1 \\
ST3 (11) & $0 \pm 0.08$ & 1 & $0 \pm 0.10$ & 1 \\
ST4 (32) & $0 \pm 0.08$ & 1 & $0 \pm 0.11$ & 1 \\
NH1, SBES (72) & $-0.12 \pm 0.15$ & 0.93 & $-0.04 \pm 0.13$ & 0.96 \\
NH1, MBES (72) & $0.00 \pm 0.16$ & 0.92 & $0.00 \pm 0.15$ & 0.89 \\
NH2 (24) & $0 \pm 0.09$ & 1 & $0 \pm 0.12$ & 1 \\
NH3 (92) & $0 \pm 0.09$ & 1 & $0 \pm 0.14$ & 1 \\
WR1 (18) & $0.02 \pm 0.04$ & 0.28 & $0.12 \pm 0.04$ & $0.99(!)$ \\
WIJ1-2 (120) & $0.00 \pm 0.03$ & 0.57 & $0.05 \pm 0.03$ & $0.52(!)$ \\
NT1 (14) & $0.00 \pm 0.01$ & 0.15 & $0.00 \pm 0.01$ & 0.26 \\
\hline \hline
\end{tabular}

areas that show migration (Maas West, IJgeul Approach, and IJgeul Approach anchorage) have a low correlation $\rho^{(\mathrm{S})}$ between time and the overall shallowest likely depth per subarea. Apparently, their overall shallowest likely depth values change independent of time.

The MESR values are also close to zero, except for the three areas that show migration. The migration makes the shallowest likely depth values at one side of the sand waves decrease. The correlation $\rho^{(\mathrm{M})}$ between time and the shallowest likely depth is large, except for the flat, shallow area in the the TE-TSS and the two areas in the region West of IJmuiden. For those two areas (IJgeul Approach and IJgeul Approach anchorage), sand wave migration is detected, which is neither correlated with depth nor with sand wave shape. (See Chapter 5.)

For the area Maas West, in the region West of Rotterdam, migration is detected that correlates with both depth and sand wave shape. (See Chapter 4.) For this area, we find a large correlation $\rho^{(\mathrm{M})}$ between time and the shallowest likely depth for the nodes where the MESR is found, but a low correlation $\rho^{(\mathrm{S})}$ between time and the overall shallowest likely depth values. This difference between the migrating areas Maas West and the two IJgeul Approach areas is probably due to the less structured migration of the latter areas, as indicated by the differences in their correlations with depth and sand wave shape. 
Table 6.4: Overall spatial test quotients $\underline{q}^{(\mathrm{o}, \mathrm{m})}[-]$ and survey-averaged estimated morphological variance $\overline{\hat{\sigma}}^{(\mathrm{m}) 2}\left[\mathrm{~m}^{2}\right]$ per area, averaged over all subareas. The area codes are given in Table 6.2. To compare these results, the used number of SBES surveys $S^{\downarrow}$ and the used number of MBES surveys $S^{\downarrow}$ are given as well. Both the one-dimensional grid line results and the two-dimensional grid results are given. The shallow part of the TE-TSS (NT1), marked with $(*)$, has a one-dimensional and a two-dimensional overall test quotient larger than 1 for one subarea only, and therefore only one survey-averaged morphological variance for the one-dimensional analysis, and one for the two-dimensional analysis.

\begin{tabular}{lrrrrrrrr}
\hline \hline area (\# subareas) & \multicolumn{4}{c}{$\bar{q}^{(\mathrm{o}, \mathrm{m})}$} & \multicolumn{3}{c}{$\overline{\hat{\sigma}}^{(\mathrm{m}) 2}$} & \multicolumn{3}{c}{$S^{\downarrow} S^{\downarrow \downarrow}$} \\
& $1 \mathrm{D}$ & 2D & 1D & 2D & & & \\
\hline ST1, 1991-1999 (25) & 3.49 & 4.08 & 0.94 & 0.75 & 8 & 0 & \\
ST1, 2000-2002 (25) & 6.15 & 7.49 & 1.03 & 0.91 & 5 & 2 & \\
ST1, 2003-2006 (25) & 8.85 & 11.01 & 1.15 & 1.12 & 3 & 4 & \\
ST2 (20) & 5.66 & 7.73 & 3.34 & 3.28 & 6 & 0 & \\
ST3 (11) & 5.50 & 6.19 & 2.10 & 1.79 & 6 & 0 & \\
ST4 (32) & 9.43 & 13.18 & 1.81 & 1.86 & 2 & 1 & \\
NH1, SBES (72) & 3.26 & 4.92 & 1.35 & 1.33 & 4 & 0 & \\
NH1, MBES (72) & 21.55 & 31.80 & 2.34 & 3.23 & 0 & 6 & \\
NH2 (24) & 22.36 & 30.36 & 2.33 & 2.94 & 1 & 5 & \\
NH3 (92) & 15.13 & 21.91 & 1.56 & 1.92 & 0 & 7 & \\
WR1 (18) & 4.47 & 6.10 & 0.47 & 0.49 & 4 & 1 & \\
WIJ1-2 (120) & 6.07 & 9.82 & 0.48 & 0.35 & 4 & 2 & \\
NT1 (14) & 0.30 & 0.28 & 0.011 & 0.005 & 3 & 1 & $(*)$ \\
\hline \hline
\end{tabular}

\subsubsection{The two regularity parameters}

\section{The overall test quotient}

An overview of the values of the overall test quotients is given in Table 6.4. The table illustrates that, besides the area without sand waves North of Terschelling, few overall test quotients are smaller than one, and therefore almost all subareas of the other areas are classified as irregular.

It is also clear from Table 6.4 that the overall test quotients strongly depend on the type of surveys: MBES surveys result in higher values than SBES surveys. The Kriging uncertainty of the SBES surveys makes their variances larger, and thereby the test quotients smaller, as they give the size of the residuals relative to the variances and covariances of the error model. Therefore, we only conclude that all subareas are irregular, but we cannot compare regions in terms of their degree of irregularity.

A third observation is that the two-dimensional overall test quotients are larger than the one-dimensional overall test quotients. This difference in value 
is caused by the difference in redundancy $M-N$, also see equation (6.8). The two-dimensional analysis uses the same number of depth values $M$ to estimate fewer parameters $N$. This means that the estimated residuals $\underline{\hat{\mathbf{r}}}$ are larger, and thereby the overall test quotients $\underline{q}^{(\mathrm{o}, \mathrm{m})}$ are larger as well. Only in a few cases the increase in size of the residuals is so small that the increase in redundancy $M-N$ dominates equation (6.8), and the two-dimensional overall test value is smaller. The shallow part of the TE-TSS, without a sand wave pattern, is an example of such a case.

\section{The morphological variance}

We see that the type of echo-sounder also influences the morphological variances $\underline{\hat{\hat{\sigma}}}^{(\mathrm{m}) 2}$, most notably for the Noordhinder Junction. A part of the morphological variation will be incorrectly accounted for by the error variances and covariances. An error description that allows for larger residuals is able to account for more morphological variation as well.

However, another effect now dominates, which becomes clear by comparing the morphological variances of the short wavelength Critical area (A: 150 to 300 m) with those of the intermediate wavelength Critical areas (C, D and K: 200 to $400 \mathrm{~m}$ ) and those of the long wavelength Critical areas (B, E, F, G, H, I, J: 450 to $900 \mathrm{~m}$ ). Larger wavelengths apparently allow for larger deviations from a sine function. This difference is visible in the values for both the one-dimensional and the two-dimensional values, which confirms that this effect is caused by wave irregularity (deviation from a sine by peakedness or skewness in the $x$ direction), not by crest irregularity (sand wave pattern changes in amplitude or position along the $y$-direction).

The one-dimensional morphological variances can be both larger or smaller than the two-dimensional values. One effect has already been mentioned for the overall test quotients: the estimated residuals $\underline{\hat{\mathbf{r}}}$ are larger for the twodimensional situation, which also means larger estimates for morphological variance. Another effect is the acceptance of spatial extensions to the characterization. The larger redundancy in the two-dimensional analysis gives larger test quotients $\underline{q}_{a}$ for the alternative extensions $a$, which means that it is more likely that an extension is accepted. This implies that the estimated residuals $\underline{\hat{\mathbf{r}}}$ are smaller for the two-dimensional situation.

It depends on the crest irregularity of the area which effect dominates. For a relatively small degree of irregularity, the extra flexibility of the onedimensional analysis does not approximate the morphology much better than the two-dimensional analysis, the estimated residuals are not much smaller. If the larger redundancy of the two-dimensional analysis is the cause of the acceptance of a spatial extension that was not accepted for the one-dimensional analysis, the two- dimensional residuals are smaller, and the size of the added morphological variance as well. For a relatively large degree of irregularity, on the other hand, the one-dimensional analysis is able to approximate the pattern much better because it is more flexible. The two-dimensional residuals are larger, and the size of the added morphological variance as well. 
For example, the areas in the region West of IJmuiden have a relatively large one-dimensional morphological variance, indicating that the sand waves have a low crest irregularity. The areas in the Noordhinder region have a larger twodimensional morphological variance, indicating a large crest irregularity. An exception are the SBES surveys of the Noordhinder Junction. The track direction usually corresponds to the $x$-direction of the pattern, and the interpolation procedure therefore artificially creates regular structures the $y$-direction of the crest. This decreases the two-dimensional morphological variance to the size of the one-dimensional morphological variance.

\subsubsection{The two minimal detectable biases}

Table 6.5 shows the minimal detectable biases for a depth outlier during the next survey, with a power $\gamma$ of $95 \%$. With this Table, we can now conclude from the two-dimensional MDB-s that there is no large risk of missing largescale dynamics of a size of S44 order 1 for depth uncertainty. However, as we can conclude from the one-dimensional MDB-s, there is a higher risk that smallscale dynamics of that size will be missed for all regions, except for the region North of Terschelling.

The MDB-s are especially large for the regions Selected Track and Noordhinder, because the morphological variances of those regions are also large (Table 6.5), and in spite of their larger S44 critical values due to their deeper bed levels. The regions West of Rotterdam and West of IJmuiden already had a higher recommended priority, because of their MESR values. Now, we also recommend to give the regions Selected Track and Noordhinder priority over the region North of Terschelling, as indicated by a (!) in Table 6.5.

\subsection{Results: suggested improvements to the resurvey policy}

All analyzed areas under the responsibility of the Netherlands Hydrographic Service have the same resurvey category 1, with a frequency of once every two years, see Table 6.6. Although the analyzed areas in the region Noordhinder fall under the responsibility of Rijkswaterstaat North Sea, there are also category 1 areas of the Netherlands Hydrographic Service in this region. The two regions that have the largest dynamics with respect to the resurvey frequency are West of Rotterdam and West of IJmuiden. (See the MESR values in Table 6.3.) We suggest to change the current resurvey policy by assigning areas in those two regions a higher resurvey frequency than areas for which the other four factors are equal, but that are located in another region.

Also, the absence of any large indicator values for the region North of Terschelling suggest that the high resurvey frequency of the shallow part of the TSS could not be justified, unless other factors require so. The recommendations and the reasons for them are given per priority in Table 6.7 . 
Table 6.5: Minimal detectable biases [m] for a depth outlier during the next survey, with a power $\gamma$ of $95 \%$. The area codes are given in Table 6.2. All listed details per area are averages over the subareas: measurement and interpolation variance $\underline{\hat{\sigma}}^{\text {(v)2 }}$ $\left[\mathrm{m}^{2}\right]$; number of grid nodes $\sqrt{ } \bar{P}$ per grid line, assuming square grids; and number of surveys $\bar{S}$. The morphological variance $\overline{\hat{\sigma}}^{(\mathrm{m}) 2}\left[\mathrm{~m}^{2}\right]$ is taken from Table 6.4. The MDB-s are compared to the values for uncertainty of depth error $1.96 \sigma^{(\mathrm{e})}$ [m] of S44, order 1 , at a $95 \%$ confidence level [International Hydrographic Organization, 2008a]. The S44 uncertainty values are calculated using bed level $\underline{d}^{(\mathrm{b})}[\mathrm{m}]$. The areas that were not given a higher priority in Table 6.3, and have a higher one-dimensional MDB than $1.96 \sigma^{(\mathrm{e})}$ of $\mathrm{S} 44$, order 1 , are marked with (!).

\begin{tabular}{lccccccccc}
\hline \hline area & \multicolumn{3}{c}{ area details } & \multicolumn{3}{c}{ MDB } & \multicolumn{3}{c}{ S44, order 1 } \\
(\# subareas) & $\underline{\hat{\hat{\sigma}}}^{(\mathrm{v}) 2}$ & $\sqrt{ } \bar{P}$ & $\bar{S}$ & $\underline{\underline{d}}^{(\mathrm{b})}$ & 1D & $2 \mathrm{D}$ & $1.96 \sigma^{(\mathrm{e})}$ & \\
\hline ST1, 1991-1999 (25) & 0.40 & 22 & 6 & 34.5 & 0.96 & 0.19 & 0.67 & $(!)$ \\
ST1, 2000-2002 (25) & 0.41 & 22 & 5 & 34.5 & 1.01 & 0.21 & 0.67 & $(!)$ \\
ST1, 2003-2006 (25) & 0.18 & 22 & 5 & 34.6 & 0.97 & 0.20 & 0.67 & $(!)$ \\
ST2 (20) & 1.05 & 18 & 6 & 39.4 & 1.92 & 0.45 & 0.72 & $(!)$ \\
ST3 (11) & 0.49 & 20 & 6 & 36.0 & 1.40 & 0.29 & 0.68 & $(!)$ \\
ST4 (32) & 0.14 & 25 & 3 & 41.2 & 1.16 & 0.24 & 0.73 & $(!)$ \\
NH1, SBES (72) & 0.67 & 23 & 4 & 37.1 & 1.19 & 0.25 & 0.69 & $(!)$ \\
NH1, MBES (72) & 0.12 & 23 & 6 & 37.6 & 1.27 & 0.31 & 0.70 & $(!)$ \\
NH2 (24) & 0.12 & 26 & 6 & 36.0 & 1.19 & 0.26 & 0.68 & $(!)$ \\
NH3 (92) & 0.11 & 20 & 6 & 34.2 & 1.12 & 0.27 & 0.67 & $(!)$ \\
WR1 (18) & 0.08 & 25 & 5 & 20.2 & 0.59 & 0.12 & 0.56 & \\
WIJ1-2 (120) & 0.11 & 22 & 6 & 25.4 & 0.64 & 0.12 & 0.60 & \\
NT1 (14) & 0.08 & 16 & 4 & 19.6 & 0.30 & 0.08 & 0.56 & \\
\hline \hline
\end{tabular}

Table 6.6: Existing situation of resurvey categories per region. The geographic distribution of the categories over the BNLCS and the corresponding resurvey frequencies are shown in Figure 6.1.

\begin{tabular}{lcc}
\hline \hline region & highest current & recommended \\
& resurvey category & new priority \\
\hline West of Rotterdam (WR) & category 1 & 2 \\
West of IJmuiden (WIJ) & category 1 & 2 \\
Selected Track (ST) & category 1 & 4 \\
Noordhinder (NH) & category 1 & 4 \\
North of Terschelling (NT) & category 1 & 5 \\
\hline \hline
\end{tabular}


Table 6.7: Recommended new situation: differing recommended priorities per region.

\begin{tabular}{cll}
\hline \hline $\begin{array}{c}\text { recommended region } \\
\text { new priority }\end{array}$ & reason \\
\hline 1 & - & \\
2 & West of Rotterdam (WR), & \\
& West of IJmuiden (WIJ) & large MESR \\
3 & - & large 2D MDB \\
4 & Selected Track (ST), & Noordhinder (NH) \\
& large 1D MDB \\
5 & North of Terschelling (NT) & no large indicators \\
\hline \hline
\end{tabular}

\subsection{Discussion}

\subsubsection{Shallowest likely depth as a hydrographic concept}

Our concept of the shallowest likely depth is algorithmic, just like the concept of the navigation surface [Smith, 2003; Smith et al., 2002], but the way it deals with small-scale morphology is different. Instead of defocusing isobaths around the shallowest values, we add the residual morphology to the uncertainty of the schematic characterization, and apply a depth reduction by creating a full grid of shallowest likely depth values, at a confidence level that is set as considered necessary. From such a grid, it should be possible to draw non-shoal biased isobaths that have a constant confidence level. The automatic generation of unbiased isobaths is more straightforward than the application of a defocusing algorithm, even if a scale-dependent generalization is necessary.

In a cleaned MBES survey, i.e. after the removal of gross errors and objects, the extreme values are often generated by the least accurate outer beams of the echo sounder swath. In that case, classical shoal biasing selects those least accurate values for chart production. An advantage of our concept is that it does not rely on the shallowest values, but uses a surface $\underline{\hat{m}}_{97.5 \%}(x, y)$ that is estimated from all depth values and that has a specified confidence level. This reduces the importance of an advanced data cleaning procedure.

\subsubsection{Irregularity as a morphological concept}

The one-dimensional morphological variance components describe wave irregularity, without a distinction between horizontal and vertical asymmetry. Crest irregularity is included in the values of the two-dimensional morphological variance components. Crest irregularity is large if the two-dimensional morphological variance component is large, but its one-dimensional equivalent is not. Because of the relation between the morphological variance component and the MDB (equation (6.14) and Table 6.1), the MDB values contain the information on wave and crest irregularity as well. It is preferable to use the morphological 
variance components though, as they are not connected to a certain type of dynamics.

Knaapen [2005] found a correlation between the migration rate of sand waves in the Noordhinder region and shape information like their horizontal asymmetry. In Chapters 4 and 5, we used the shape-based migration predictor, using the results of a two-dimensional deformation analysis. The differences between these results and results based on a one-dimensional deformation analysis are able to provide insight into the role of crest irregularity.

\subsubsection{The four indicators}

The presence of four other factors for the resurvey policy (Section 6.1) means that changes could only be made if the interpretation of observed sea floor dynamics (factor 5) is done in combination with the interpretation of data describing the other factors. Therefore, we make recommendations on morphological priority only, and leave the formulation of adapted resurvey frequencies outside the scope of this study.

The two indicators SLDR and MESR give a powerful combination of information on the shallowest depth in an area and the maximum shoaling rate in that area. The two MDB-s add indicators on the size of potentially missed dynamics, ensuring recommendations that are robust, even in case of complicated morphology. This enables resurvey planners to make sound choices in the assignment and change of resurvey frequencies, leading to safer navigation and more efficient deployment of survey capacity.

The sand wave pattern in all four sand wave regions is irregular (See the overall spatial test quotients in Table 6.4.), i.e. the pattern differs from a onedimensional sine function that is constant in the direction along the crest. The two regions with migrating sand waves are less irregular, and the two regions without migrating sand waves are more irregular (See the morphological variances in Table 6.4.). Potentially, smaller dynamics are not detected in these areas. Change of survey frequency of the two more irregular regions, based on the generally static results of the deformation analyses of those regions, should therefore be done with care.

\subsection{Conclusion}

The results of the deformation analysis, applied to bathymetric surveys in the Southern North Sea, have been interpreted using four indicators. These indicators are the Shallowest Likely Depth Rate (SLDR), the Maximum Estimated Shoaling Rate (MESR), and the Minimal Detectable Biases (MDB-s) for the one-dimensional and the two-dimensional analyses. They summarize the estimates for the morphological parameters, and thereby finalize the data reduction in several steps, starting with a series of survey data. The irregularity of the analyzed patterns differs, which gives different sizes of dynamics that are detectable. 
In the present survey policy, all analyzed areas fall into the category with the highest resurvey frequency, or fall under the responsibility of Rijkswaterstaat North Sea. Using the indicators that we developed, the regions of the analyzed areas are ordered into several categories. The regions West of Rotterdam and West of IJmuiden should have a higher priority than the regions Selected Track and Noordhinder, which in turn should have a higher priority than the region North of Terschelling. (Also see the Tables 6.6 and 6.7. This contributes to the efficiency of the survey efforts that the Netherlands Hydrographic Office makes in the regions of the analyzed areas, making more time available to survey other areas. This in turn aids safe navigation in the Southern North Sea.

The approach to improve the resurvey policy by the analysis of a series of data is possible due to the storage of past surveys at a high resolution. The resulting recommendations for the survey plan are of higher detail than recommendations from exploratory surveys or remote sensing techniques. With the recent improvements in morphological models, these models could also become a valuable source for adaptations in resurvey frequency, especially in cases where future human intervention plays a role. 


\section{Chapter 7}

\section{Conclusions and recommendations}

\subsection{Answers to the subquestions}

The research question is answered by considering a set of subquestions, posed in Chapter 1. Each subquestion was dealt with in a separate chapter.

Q1: How can we express a bathymetric survey as a grid of depth values and their uncertainties, to be used as input for deformation analysis? (Chapter 2)

Interpolation of bathymetric data is often necessary to create a regular grid for deformation analysis. The interpolation of such data, already affected by horizontal and vertical measurement errors, results in an additional interpolation error. The application of Universal Kriging calculates a variance for this interpolation error, and is suitable for areas with a slope and a sand wave pattern.

Q2: How can deformation analysis estimate sea floor dynamics, using the appropriate sea floor parameters, based on a time series of bathymetric surveys expressed as grids of depth values and their uncertainties? (Chapter 3)

The presented deformation analysis procedure for the detection and estimation of sea floor dynamics was tested by a scenario that is realistic for the Southern North Sea: the morphology is based on measured data, and the artificially introduced morphodynamics are typical examples of dynamics in the Southern North Sea. The results of the test scenario show that a simple morphological representation can be used for the analysis of sea floor dynamics, even for the morphologically complicated area that we presented. The method gives parameter estimates for the deformations that include their uncertainties. A characterization that consists of a sloping plane and a sinusoidal wave is sufficient to analyze dynamics in tidal sand wave areas, including growth and migration of a sand wave pattern.

The procedure requires the availability of variances per depth value for the measurement and interpolation uncertainties. Several levels of significance have to be set carefully. Also, the procedure requires some prior insight into the characteristics of the area to set the search interval for the wavelength, to choose the size of the subareas, and to select the number of spatial dimensions of an analysis. 
Q3: How do estimates of parameters for sand wave pattern dynamics, obtained by the application of deformation analysis, vary on the scale of such a pattern for a specific sand wave area in the Southern North Sea? (Chapter 4)

Deformation analysis shows that the tidal sand waves in anchorage area Maas West have migration rates up to $7.5 \mathrm{~m} / \mathrm{yr}$. The migration rate is larger in the shallower subareas in the Southeast. The migration rate also depends on the shape of the sand waves. These results confirm known correlations with depth and sand wave shape, now using the uncertainty of the estimates. The migration rates are also in agreement with idealized process-based models for tidal sand waves.

Q4: How do estimates of parameters for the sea floor and its changes, obtained by the application of deformation analysis, vary over several areas in the Southern North Sea? (Chapter 5)

The bed level dynamics of the five analyzed regions are limited to several decimeters over the past two decades, except for the zero-dimensional results. In this case, the largest upward trends and outliers are usually due to sand wave migration. Large dynamics are absent, except for migration of the sand waves relatively close to the North and South Holland coasts. Migration rates correlate with bed level and shape information for only one of those two regions. No generic conclusions on the impact of human interventions can be drawn, because sand wave dynamics in the two areas with a maintained depth differ too much from each other.

Q5: How can the results of the application of deformation analysis be used to improve the resurvey policy of the Netherlands Hydrographic Service?

(Chapter 6)

The results of the deformation analysis, applied to bathymetric surveys in the Southern North Sea, have been interpreted using four indicators. These indicators are the Shallowest Likely Depth Rate (SLDR), the Maximum Estimated Shoaling Rate (MESR), and the Minimal Detectable Biases (MDB-s) for the one-dimensional and the two-dimensional analyses. They summarize the estimates for the morphological parameters, and thereby finalize the data reduction in several steps, starting with a series of survey data. The irregularity of the analyzed patterns differs, which gives different sizes of dynamics that are detectable.

In the present survey policy, all analyzed areas fall either into the category with the highest resurvey frequency, or under the responsibility of Rijkswaterstaat North Sea. The regions of the analyzed areas are ordered into several categories, using the indicators. The regions West of Rotterdam and West of IJmuiden should have a higher priority than the regions Selected track and Noordhinder, which in turn should have a higher priority than the region North of Terschelling. 


\subsection{Conclusions on the research question}

The research question, posed in Chapter 1, is as follows:

How can deformation analysis use a time series of bathymetric surveys to estimate sea floor dynamics that may include a tidal sand wave pattern in a satisfactory way, and how can these results be applied to improve a resurvey

$$
\text { policy? }
$$

In this project, an estimation was said to be satisfactory if the method detects all significant sea floor dynamics, if these dynamics are estimated using as few parameters as possible, and if the uncertainty associated with these dynamics is as small as possible. The resurvey policy was said to be improved if all detected sea floor dynamics could influence the policy, if the risk of missed sea floor dynamics is accounted for, if sea floor dynamics that have a smaller uncertainty have a larger influence, and if sea floor dynamics that pose a more severe threat to navigation have a larger influence.

Universal Kriging of bathymetric surveys results in a series of grids of depth values and their variances, and is suitable for areas with a slope and a sand wave pattern. Deformation analysis gives insight into the past behaviour of the sea floor. This combination of methods even performs well for morphologically complicated areas, using a characterization that allows for a slope and a sand wave pattern. This characterization estimates the significant parameters for the dynamics, including their uncertainties, and it limits both the number of parameters and the parameter uncertainty, if temporal extensions consisting of a trend and outlying parameters are tested. A least-squares variance component estimation is necessary to account for the residual morphology and morphodynamics.

Large dynamics are absent for the analysed areas in all five studied regions in the Southern North Sea, except for migration of the sand waves relatively close to the North and South Holland coasts. Migration rates correlate with bed level and shape information for only one of those two regions.

A set of four indicators has been set up to summarize the estimates for the morphological parameters, which completes the data reduction process. Based on these indicators, we have been able to assign different priorities to five regions of the resurvey plan that currently all have areas in the highest category. This contributes to the efficiency of the survey efforts of the Royal Netherlands Navy.

\subsection{Recommendations for further research}

We recommend that the following topics are studied further:

R1: Improve the estimation procedure by alternative sea floor representations.

Tidal sand wave patterns vary greatly in their degree of irregularity over the Southern North Sea. Additional spatial and temporal extensions could improve the representation, which keeps the residual variation small. This reduces the 
additional morphological and temporal uncertainties. This in turn allows for more accurate estimates, and smaller dynamics that are detectable.

However, only relevant parameters reduce the morphological variance sufficiently to counteract the loss of accuracy of the estimates, due to the decrease in redundancy. The redundancy is the surplus of the measurements over the number of parameters, a lower redundancy means a lower number of measurements per parameter, and therefore a lower accuracy per parameter. The inclusion of new parameters should therefore be done with caution.

For instance, the detection and estimation of potential along-crest components of sand wave migration requires that the method could estimate the crest length of sand waves. In that case, an additional parameter has to be added to the sand wave characterization, for position along the crest direction, from which an along-crest migration rate could be estimated. Sand wave migration along the wave crests was observed for the Southern North Sea by Van Dijk and Egberts [2008].

Sand wave asymmetry could be represented by additional parameters, like the addition of a second sine wave with half the wavelength. If a sand wave pattern deviates strongly from the shape of a sine wave, a better approach could be to represent sand waves using a different basic shape, like a triangular saw tooth pattern. The detection of changes in asymmetry in step 2 of the method is possible if sand wave asymmetry parameters are available in step 1.

Another example is the study of sand wave behaviour after removing the pattern partly or fully by dredging. The regeneration of a sand wave pattern for the Southern North Sea has been studied by Knaapen and Hulscher [2002]. A tidal sand wave pattern might or might not recover, and initial sand wave growth might be nonlinear. These types of behaviour will now be represented by a series of outliers. If dredging moments are available beforehand, it makes sense to design specific alternative hypotheses in step 2 of the method. One could think of a static situation before dredging and another static situation after, or a linear trend after the initial static situation. The linear trend potentially is adapted by outliers for nonlinear behaviour. A prerequisite is the accurate documentation of dredging moments.

A complication of the estimation of a wavelength using deformation analysis is that the relation between the observed depth values and wavelength is nonlinear. The optimization procedure that solves this complication allows for a constant wavelength only, both in time and over a grid or grid line. Crest irregularity could not be solved by varying the dominant wavelength over the grid or grid line. Also, it is impossible to detect change in the dominant wavelength over time. Such a change in wavelength in time could e.g. happen during the recovery of a pattern after dredging, or due to the change of flow characteristics after human intervention at sea, like the construction of dams and land reclamation. The implementation of a linearized estimation procedure will allow for the estimation of dominant wavelength as a regular parameter, allowing for its change in time. 


\section{R2: Explore application to other morphological patterns.}

Application of the designed procedure to rhythmic features on other scales and in other environments is attractive. Deformation analysis procedures already were implemented by Lindenbergh and Hanssen [2003] for an eolian sand dune field, and by Lindenbergh [2004] for megaripples at sea. Application to smallerscale variations like megaripples and ripples is hindered by the limited positional accuracy and resolution at sea. Application in a laboratory environment does not have these disadvantages.

In a fluvial environment, other algorithmic methods already are in use [McElroy et al., 2008; Sieben, 2004] that are deterministic in nature. Although measurement and interpolation uncertainties are smaller for depth measurement in rivers, the application of a stochastic method like deformation analysis will help to distinguish between measurement errors and morphological change in such an environment as well.

Large-scale features like sand banks are usually less dynamic than smaller scale features. The application of deformation analysis is still attractive for static features, to detect the dominant wavelength of complicated pattern. It offers an alternative approach to the spectral analysis of a pattern [Van Dijk et al., 2008; Smale et al., 2007].

\section{R3: Study spatial correlations of the estimates of the analyzed areas to surrounding areas.}

Classification of areas of equal morphological and physical characteristics enables insight into the spatial extent of the detected dynamics. Morphological characteristics are the depth, length, amplitude and shape of the rhythmic patterns on the sea floor on various scales. Physical characteristics are the composition of the sea floor, tidal flow, and wave climate. If the extent of areas with equal characteristics is known, careful attempts could be made to spatially extrapolate the estimates of the analyzed areas.

To improve the efficiency of the survey policy of the Netherlands Hydrographic Service, conclusions on morphodynamics need to be drawn on the scale of the Southern North Sea, instead of the scale of regions of limited spatial extent. Morphology has already been studied on this scale, recently by Van der Veen et al. [2006] and Knaapen [2009]. Morphodynamics on this scale, based on series of surveys, will be studied in a follow-up project by Deltares [2008].

R4: Study the predictive capacities of the estimates of the analyzed areas.

The approach to improve the resurvey policy by the analysis of a series of data is possible due to the storage of past surveys at a high resolution. The resulting recommendations for changes in the survey policy are of higher detail than recommendations from recent exploratory surveys or remote sensing techniques, but they are based on linear extrapolations of trends. To get a better insight into the present and future morphodynamics than by a linear extrapolation, a coupling with process-based models should be made, especially for areas where 
the indirect effect of human interventions could pose a threat to navigation [Van der Veen, 2008; Roos et al., 2008]. Such a coupling has already been made between the trend analysis method of Wüst [2004] and the process-based Landau equation for sand wave growth, by Knaapen et al. [2005].

The study of correlations between morphodynamics and sand wave shape enables the prediction of morphodynamics from a single bathymetric survey, instead of a time series [Knaapen, 2005]. Especially the correlations between wave irregularity and migration rate, and between crest irregularity and variations in migration rate deserve attention.

R5: Explore the use of results of deformation analysis in coastal zone management.

Prudent coastal zone management requires appropriate assessment procedures for the impact of human interventions into natural systems [Hommes et al., 2009; Hommes, 2008]. An example of such a procedure in the Netherlands is the Milieu Effect Rapportage (MER). One could think of large infrastructure projects like the extension of the Port of Rotterdam, sand mining and dumping, the development of offshore wind farms, and the extraction of oil and gas from the continental shelf. Deformation analysis helps the evaluation of the influence of infrastructure projects in the decades after their realization, and thereby improves the assessment of future projects.

Unexploded ordnance and mines are abundant in the Southern North Sea, mostly due to the Second World War. Most of these objects were buried in the sandy sea bed in time, due to dynamics of the sea floor, like scouring (i.e. sand removal around objects due to flow) and sand wave migration. Sea floor dynamics could not only cover objects, but also reveal them again after unpredictable time. Knowledge on sea floor dynamics helps to identify areas of higher risk of object re-exposure, which is of great value for the safety of mariners, especially fishermen. Those areas could be added as Additional Military Layers (AML-s) in geographical information systems for mine counter-measures.

\subsection{Implementation at the Netherlands Hydrographic Service}

The method developed in this study should be further implemented into the survey processing at the office of the Netherlands Hydrographic Service. The current implementation in MATLAB should be converted into a stand-alone computer program, which communicates with the bathymetric archiving system. Recommendations for changes in survey policy could then directly be sent from the data processing department to the survey planning department, without the routine intervention of the developers of the method.

It is recommended that the method is applied to other areas of the BNLCS, once more data becomes available. The results of every newly analysed area improves the analysis of morphodynamics on the scale of the BNLCS further, which will be done by Deltares [2008]. 
The transition from the recommended resurvey priorities to adapted resurvey frequencies should be done taking all five factors of Chapter 6 into account. Changes in resurvey frequency were already made for the Critical areas in the Selected Track region in 2003, based on earlier results of deformation analysis. It is now possible to make further changes in resurvey frequency for the five analysed areas. 


\section{Bibliography}

Amiri-Simkooei, A. R. [2007]. Least-Squares Variance Component Estimation - Theory and GPS Applications, PhD thesis, Delft University of Technology, Delft, The Netherlands. no. 64 in Publications on Geodesy, Netherlands Geodetic Commission, ISBN: $10-90-804147-5-1$ and 13-978-90-804147-5-4.

Amiri-Simkooei, A. R. and Tiberius, C. C. J. M. [2006]. Assessing receiver noise using GPS short baseline time series, GPS. Sol. 11: 21-35. doi: 10.1007/s10291-006-00268.

Armstrong, M. [1984]. Problems with universal kriging, Math. Geol. 16(1): 101-108.

Baarda, W. [1968]. A testing procedure for use in geodetic networks, number 9 in Publications on Geodesy, Netherlands Geodetic Commission, Delft, The Netherlands.

Besio, G., Blondeaux, P., Brocchini, M., Hulscher, S. J. M. H., Idier, D., Knaapen, M. A. F., Németh, A. A., Roos, P. C. and Vittori, G. [2008]. The morphodynamics of tidal sand waves: a model overview, Coastal Engineering 55: 657-670. doi: 10.1016/j.coastaleng.2007.11.004.

Besio, G., Blondeaux, P., Brocchini, M. and Vittori, G. [2004]. On the modelling of sand wave migration, J. Geophys. Res. 109(C04108). doi: 10.1029/2002JC001622.

Bottelier, P., Briese, C., Hennis, N., Lindenbergh, R. C. and Pfeifer, N. [2005]. Distinguishing features from outliers in automatic kriging-based filtering of MBES data: a comparative study, in P. Renard, H. Demougeot-Renard and R. Froidevaux (eds), GeoENV V - Geostatistics for environmental applications, Springer, Berlin, Germany, pp. 403-414.

Bouwmeester, E. and Heemink, A. [1993]. Optimal line spacing in hydrographic survey, Int. Hydro. Rev. 70(1): 37-48.

Bowyer, J. K. [1992]. Basin changes in Jervis Bay, New South Wales: 1894-1988, Mar. Geol. 105: 211-224.

Buijsman, M. C. [2007]. Ferry-observed variability of currents and bedforms in the Marsdiep inlet, $\mathrm{PhD}$ thesis, Utrecht University, Utrecht, The Netherlands. ISBN: 978-90-393-4613-6.

Burton, B. W. [1977]. An investigation of a sandwave field at the south western end of Sandettié Bank, Dover Strait, Int. Hydro. Rev. 54(2): 46-59.

Calder, B. [2003]. Automatic statistical processing of multibeam echosounder data, Int. Hydro. Rev., New Series 4(1): 53-68. 
Calder, B. [2006]. On the uncertainty of archive hydrographic surveys, IEEE J. of Oceanic Engineering 31(2): 249-265. doi: 10.1109/JOE.2006.872215.

Calkoen, C. J., Hesselmans, G. H. F. M. and Wensink, G. J. [1998]. The use of radar imagery to assess the bottom topography of shallow seas, Int. Hydro. Rev. 75(2): 43-50.

Chatfield, C. [1989]. The analysis of time series, 4 edn, Chapman \& Hall, London, UK.

Chilès, J. [1976]. How to adapt kriging to non-classical problems: three case studies, in M. Guarascio, M. David and C. Huijbregts (eds), Advanced geostatistics in the mining industry - Proceedings of the NATO advanced study institute, Rome, Italy, Vol. 24 of $C$, Reidel Publishing Company, Dordrecht, The Netherlands, pp. 69-89.

Chilès, J. and Chauvet, P. [1975]. Kriging: a method for cartography of the sea floor, Int. Hydro. Rev. 52(1): 25-41.

Chilès, J.-P. and Delfiner, P. [1999]. Geostatistics, Modeling Spatial Uncertainty, Probability and Statistics, John Wiley \& Sons, New York, USA.

Christakos, G. [1984]. On the problem of permissable covariance and variogram models, Water Resources Res. 20: 251-265.

Chrzanowski, A. [2006]. Tasks and achievements of the FIG working group on deformation measurements and analysis, in H. Kahmen and A. Chrzanowski (eds), Proceedings of the 3rd IAG symposium on Geodesy for geotechnical and structural engineering and 12th FIG symposium on deformation measurement, Baden, Austria, Vienna University of Technology, Vienna, Austria.

Cressie, N. and Kornak, J. [2003]. Spatial statistics in the presence of location error with an application to remote sensing of the environment, Stat. Sci. 18(4): 436-456.

Davis, J. C. [2002]. Statistics and Data Analysis in Geology, 3 edn, John Wiley \& Sons, New York, USA.

De Haan, F. P. J. [1996]. Onderzoek TE-route CAT I gebied, Technical report, Hydrographic Service of the Royal Netherlands Navy, The Hague, The Netherlands.

De Heus, H., Joosten, P., Martens, M. and Verhoef, H. [1994]. Stability-analysis as part of the strategy for the analysis of the Groningen gasfield levellings, in H. Papo (ed.), Proceedings of the Perelmutter workshop on dynamic deformation models, Haifa, Israel, 29 August - 1 September 1994.

De Koning, M. F. [2007]. The stochastic characteristics of geometric properties of sand waves in the North Sea, Master's thesis, University of Twente, Enschede, The Netherlands.

De Oliveira, S. S., Mandarino, F. and De Souza, F. J. [2007]. FIS: resurvey decision system - using fuzzy inference systems for area selection, Hydro Int. 11(3): 31-34.

Dehling, T. [2006]. Determining survey frequency and resolution - BSH concept and realisation, Hydro Int. 10(2): 7-9. 
Deltares [2008]. Research design for the scientific validation of the hydrographic survey policy of the hydrographic office of the royal netherlands navy, Technical report, Deltares, Subsurface and Ground water systems department, Utrecht, The Netherlands.

Dienst der Hydrografie [2007]. Risicoanalyse achterstanden hydrografische opnemingen, peildatum 1 oktober 2007, Technical report, Hydrographic Service of the Royal Netherlands Navy, The Hague, The Netherlands.

Dorst, L. L. [2003]. Zeebodemmonitoring met geostatistiek en deformatieanalyse, Technical report, Koninklijke Marine, Dienst der Hydrografie, The Hague, The Netherlands.

Dorst, L. L. [2004]. Survey plan improvement by detecting sea floor dynamics in archived echo sounder surveys, Int. Hydro. Rev., New Series 5(2): 49-63.

Dorst, L. L. [2005]. Optimisation of survey frequency - the Netherlands Hydrographic Service, Hydro Int. 9(8): 6-9.

Dorst, L. L. and Roos, P. C. [2008]. Bathymetric applications of geostatistics, Technical Report 2008R002/WEM-002, Hydrographic Service of the Royal Netherlands Navy, and Water Engineering and Management group of the University of Twente.

Dorst, L. L., Roos, P. C. and Hulscher, S. J. M. H. [2007]. Estimation of sand wave dynamics in the Southern North Sea, in J. McKee Smith (ed.), Proceedings of the 30th International Conference on Coastal Engineering 2006, World Scientific, pp. 26302642 .

Duffy, G. P. and Hughes-Clarke, J. E. [2005]. Application of spatial cross correlation to detection of migration of submarine sand dunes, J. Geophys. Res. 110(F04S12). doi: 10.1029/2004JF000192.

Fritsch, F. N. and Carlson, R. E. [1980]. Monotone piecewise cubic interpolation, SIAM J. Numerical Analysis 17: 238-246.

Gabrosek, J. and Cressie, N. [2002]. The effect on attribute prediction of location uncertainty in spatial data, Geogr. Analysis 34: 263-285.

Gillissen, H. and Pulles, S. W. P. [1996]. Vergelijking surveys POL-gebied mei 1995 - juli 1996, Technical report, Hydrographic Service of the Royal Netherlands Navy, The Hague, The Netherlands.

Hare, R. [1995]. Depth and position error budgets for multibeam echosounding, Int. Hydro. Rev. 72(2): 37-69.

Heap, B. [2007]. Mariner perceptions regarding the display of uncertainty on nautical charts, Master's thesis, University of New Hampshire, Durham, USA.

Herzfeld, U. C. [1989]. Variography of submarine morphology: problems of deregularization, and cartographical implications, Math. Geol. 21(7): 693-713.

Hommes, S. [2008]. Conquering complexity : dealing with uncertainty and ambiguity in water management, $\mathrm{PhD}$ thesis, University of Twente, Enschede, The Netherlands. ISBN: 978-90-365-2742-2. 
Hommes, S., , Mulder, J. P. M., Otter, H. and Bressers, H. T. A. [2009]. Role of perceptions and knowledge in the impact assessment for the extension of Mainport Rotterdam, Marine Policy 33(1): 146-155.

Hulscher, S. J. M. H. [1996]. Tidal-induced large-scale regular bed form patterns in a three-dimensional shallow water model, J. Geophys. Res. 101(C9): 20,727-20,744.

Hulscher, S. J. M. H. and Van den Brink, G. M. [2001]. Comparison between predicted and observed sand waves and sandbanks in the North Sea, J. Geophys. Res. 106(C5): 9327-9338.

International Hydrographic Organization [2007]. IHO transfer standard for digital hydrographic data, Technical Report Special publication 5\%, International Hydrographic Bureau, Monaco.

International Hydrographic Organization [2008a]. IHO standards for hydrographic surveys, Technical Report Special publication 44, 5th ed., International Hydrographic Bureau, Monaco.

International Hydrographic Organization [2008b]. Status of hydrographic surveying and nautical charting worldwide, Technical Report Special publication 55, 3rd ed., International Hydrographic Bureau, Monaco.

Isaaks, E. H. and Srivastava, R. H. [1989]. Applied Geostatistics, Oxford university press, New York, USA.

Johnson, P. [2004]. ZOCMAN cometh, or the application of Zones of Confidence in the Australian Hydrographic Service, Int. Hydro. Rev., New Series 5(3): 6-16.

Jones, N. S., Kain, J. M. and Stride, A. H. [1965]. The movement of sand waves on Warts Bank, Isle of Man, Mar. Geol. 3: 329-336.

Kember, I. D. [1984]. How often? - towards an optimum survey interval for mobile seabeds, Int. Hydro. Rev. 61(1): 55-79.

Kielland, P., Burrows, K., Ward, B., Dagbert, M. and Velberg, R. [1992]. Towards IHOstat: IHO approved software which evaluates the quality of bathymetric data, Proceedings of Hydro 92, Copenhagen, The Hydrographic Society, Plymouth, United Kingdom.

Kielland, P. and Dagbert, M. [1992]. The use of spatial statistics in hydrography, Int. Hydro. Rev. 69(1): 71-92.

Kielland, P. and Tubman, T. [1994]. On estimating map model errors and GPS position errors: (applying more science to the art of mavigation), Int. Hydro. Rev. 71(2): 4767.

Knaapen, M. A. F. [2004]. Measuring sand wave migration in the field. comparison of different data sources and an error analysis, in S. J. M. H. Hulscher, T. Garlan and D. Idier (eds), Proceedings of the 2nd international workshop on Marine Sand Wave and River Dune Dynamics, Enschede, The Netherlands, University of Twente, Enschede, The Netherlands. 
Knaapen, M. A. F. [2005]. Sandwave migration predictor based on shape information, J. Geophys. Res. 110(F04S11). doi: 10.1029/2004JF000195.

Knaapen, M. A. F. [2009]. Sandbank occurence on the Dutch continental shelf in the North Sea, Geo-Mar. Lett. 29: 17-24. doi: 10.1007/s00367-008-0105-7.

Knaapen, M. A. F. and Hulscher, S. J. M. H. [2002]. Regeneration of sand waves after dredging, Coastal Engineering 46: 277-289.

Knaapen, M. A. F., Hulscher, S. J. M. H. and De Vriend, H. J. [2001]. A new type of sea bed waves, Geophys. Res. Lett. 28(7): 1323-1326.

Knaapen, M. A. F., Hulscher, S. J. M. H., Tiessen, M. C. H. and Van den Berg, J. [2005]. Using a sand wave model for optimal monitoring of a navigation depth, in G. Parker and M. H. García (eds), Proceedings of the 4th IAHR symposium on River, Coastal and Estuarine Morphodynamics, Urbana, Illinois, USA, Taylor \& Francis, London, UK.

Koch, K.-R. [1999]. Parameter estimation and hypothesis testing in linear models, 2nd edn, Springer-Verlag, Berlin, Germany.

Laban, C. [2005]. Sea bed resources and hydrography — exploration of seabed resources using bathymetry and geology, Hydro Int. 9(9): 30-33.

Lafon, F., De Melo Apoluceno, D., Dupuis, H., Michel, D., Howa, H. and Froidefond, J. M. [2004]. Morphodynamics of nearshore rhythmic sandbars in a mixed-energy environment (SW France): I. mapping beach changes using visible satellite imagery, Estuarine, Coastal and Shelf Science 61: 289-299.

Lanckneus, J., De Moor, G. and Stolk, A. [1994]. Environmental setting, morphology and volumetric evolution of the Middelkerke Bank (southern North Sea), Mar. Geol. 121: $1-21$.

Langeraar, W. [1966]. Sand waves in the North Sea, Hydrogr. Newsletter 1(5): 243-246.

Langhorne, D. N. [1982a]. The stability of the top metre of the sea bed: Its importance to engineering and navigational projects, Int. Hydro. Rev. 59: 80-94.

Langhorne, D. N. [1982b]. A study of the dynamics of a marine sandwave, Sedimentology 29: 571-594.

Le Bot, S., Idier, D., Garlan, T., Trentesaux, A. and Astruc, D. [2000]. Dune dynamics: from field measurements to numerical modelling - application to bathymetric survey frequency in the Calais-Dover straight, in A. Trentesaux and T. Garlan (eds), Proceedings of the international workshop on Marine Sand Dynamics, Lille, France, University of Lille 1, Lille, France.

Lehmann, E. L. [1986]. Testing statistical hypotheses, 2nd edn, John Wiley \& Sons, New York, USA.

Lindenbergh, R. C. [2004]. Parameter estimation and deformation analysis of sand waves and mega ripples, in S. J. M. H. Hulscher, T. Garlan and D. Idier (eds), Proceedings of the 2nd international workshop on Marine Sand Wave and River Dune Dynamics, Enschede, The Netherlands, University of Twente, Enschede, The Netherlands. 
Lindenbergh, R. C., Dorst, L. L., Wüst, J. C. and Menting, P. G. [2007]. Simulation, detection and prediction of sea floor dynamics, Int. Hydro. Rev., New Series 8(2): $25-36$.

Lindenbergh, R. C. and Hanssen, R. F. [2003]. Eolian deformation detection and modeling using airborne laser altimetry, Proceedings of the 2003 IEEE International Geoscience and Remote Sensing Symposium, Toulouse, France, IEEE International. ISBN: 0-7803-7929-2.

Lindenbergh, R. C., Van Dijk, T. A. G. P. and Egberts, P. J. P. [2006]. Separating bedforms of different scales in echo sounding data, Proceedings of Coastal Dynamics 2005, Barcelona, Spain, American Society of Civil Engineers. CD-ROM.

McElroy, B., Mohrig, D. and Blom, A. [2008]. Determining characteristic scales for the dynamics and geometry of sandy bedforms, in D. Parsons, T. Garlan and J. Best (eds), Proceedings of the 3rd international workshop on Marine Sand waves and River Dune Dynamics, Leeds (UK), University of Leeds, pp. 219-225.

Monahan, D. [2007]. GEBCO and deep water - data assembler, converter, interpreter and disseminator, Hydro Int. 11(3).

Németh, A. A., Hulscher, S. J. M. H. and De Vriend, H. J. [2002]. Modelling sand wave migration in shallow shelf seas, Continental Shelf Res. 22: 2795-2806.

Németh, A. A., Hulscher, S. J. M. H. and Van Damme, R. M. J. [2007]. Modelling offshore sand wave evolution, Continental Shelf Res. 27: 713-728. doi: 10.1016/j.csr.2006.11.010.

NOAA Office of Coast Survey [2008]. NOAA hydrographic survey priorities, Technical report, National Oceanic and Atmospheric Administration.

Pluymaekers, S. J. A. [2007]. A data analysis to bed dynamics in the western scheldt estuary, Master's thesis, Delft University of Technology, Delft, The Netherlands.

Pluymaekers, S., Lindenbergh, R. C., Simons, D. and De Ronde, J. [2007]. A deformation analysis of a dynamic estuary using two-weekly MBES surveying, Proceedings of Oceans0\%.

Pyrcz, M. J. and Deutsch, C. [2003]. The whole story on the whole effect, Newsletter of the Geostatistical Association of Australasia 18: 3-5.

RIKZ [1997]. Gebruikershandleiding DIGIPOL, Technical report, Rijkswaterstaat, Rijksinstituut voor Kust en Zee, The Hague, The Netherlands.

Robert, A. [1988]. Statistical properties of sediment bed profiles in alluvial channels, Math. Geol. 20(3): 205-225.

Robert, A. and Richards, K. S. [1988]. On the modelling of sand bedforms using the semivariogram, Earth Surface Processes and Landforms 13: 459-473.

Roos, P. C., Hulscher, S. J. M. H. and De Vriend, H. J. [2008]. Modelling the morphodynamic impact of offshore sandpit geometries, Coastal Engineering 55: 704-715. doi: $10.1016 /$ j.coastaleng.2008.02.019. 
Salsman, G. G., Tolbert, W. H. and Villars, R. G. [1966]. Sand-ridge migration in St. Andrew Bay, Florida, Mar. Geol. 4: 11-19.

Scheele, C. A. [1986]. Analyse op-8 opnemingen tussen feb.'84 en nov.'85, Technical report, Hydrographic Service of the Royal Netherlands Navy, The Hague, The Netherlands.

Sieben, A. [2004]. Characterization of bed levels with statistical equivalency, in S. J. M. H. Hulscher, T. Garlan and D. Idier (eds), Proceedings of the 2nd international workshop on Marine Sand Wave and River Dune Dynamics, Enschede, The Netherlands, University of Twente, Enschede, The Netherlands.

Slobbe, C. [2005]. Interpolatie, meetnetconfiguratie en onzekerheid, Master's thesis, Hogeschool van Utrecht, Utrecht, The Netherlands.

Smale, A. J., Bijker, R. and Klopman, G. [2007]. Prediction of sand wave migration with a non-linear spectral model, Proceedings of the 5th IAHR symposium symposium on River, Coastal $\& 3$ Estuarine Morphodynamics, Enschede, The Netherlands, Taylor \& Francis, London, UK.

Smith, D. [1986]. Bodemvariabiliteit van zandgolfkammen in het traject $\mathrm{j}-\mathrm{k}$ (de aanloopgeul is $36 \mathrm{deg}$ ) van de diepwater route "Sandettie tot Noord Hinder", Technical Report GWAO 86.222, Directorate-General for Public Transport and Water Management, The Netherlands.

Smith, S. M. [2003]. The navigation surface - a multipurpose bathymetric database, Master's thesis, University of New Hampshire, Durham, USA.

Smith, S. M., Alexander, A. and Armstrong, A. A. [2002]. The navigation surface: a new database approach to creating multiple products from high-density surveys, Int. Hydro. Rev., New Series 3(2): 12-26.

Sterlini, F., Hulscher, S. J. M. H. and Hanes, D. M. [2009]. Simulating and understanding sand wave variation: A case study of the Golden Gate sand waves, $J$. Geophys. Res. 114(F02007). doi: 10.1029/2008JF000999.

Stuart, A., Ord, J. K. and Arnold, S. [1999]. Classical inference and the linear model, number $2 \mathrm{~A}$ in Kendall's advanced theory of statistics, Arnold, London, UK.

Su, H., Liu, H. and Heyman, W. D. [2008]. Automated derivation of bathymetricn information from multi-spectral satellite imagery using a non-linear inversion model, Marine Geodesy 31: 281-298.

Swart, L. M. T., Vogelzang, J., Wensink, G. J., Groenewoud, P., Hendriks, L. F. G. M., Nass, N. and Van der Lee, W. T. B. [2006]. Towards implementation of the BAS within Rijkswaterstaat, Technical Report AGI-2006-GAB-003, Ministry of Transport, Public Works and Water Management of the Netherlands, Rijkswaterstaat, Delft, The Netherlands.

Te Stroet, C. B. M. and Snepvangers, J. J. C. [2005]. Mapping curvilinear structures with local anisotropy, Math. Geol. 37(6): 635-649.

Terwindt, J. H. J. [1971]. Sand waves in the southern bight of the North Sea, Mar. Geol. 10: 51-67. 
Teunissen, P. J. G. [2000]. Adjustment theory, an introduction, Series on Mathematical Geodesy and Positioning, Delft University Press, Delft, The Netherlands.

Teunissen, P. J. G. [2001]. Testing theory, an introduction, Series on Mathematical Geodesy and Positioning, Delft University Press, Delft, The Netherlands.

Teunissen, P. J. G. and Amiri-Simkooei, A. R. [2008]. Least-squares variance component estimation, J. of Geodesy 82: 65-82. doi: 10.1007/s00190-007-0157-x.

Van den Berg, J. and Van Damme, R. [2005]. Sand wave simulations on large domains, in G. Parker and M. Garcia (eds), Proceedings of the 4th IAHR symposium on River, Coastal $\&$ Estuarine Morphodynamics, Urbana, Illinois, United States, University of Illinois at Urbana-Champaign, Urbana, Illinois, United States.

Van der Veen, H. H. [2008]. Natural and human induced seabed evolution - The occurence of large-scale bed patterns and the effects of human activities on the North Sea seabed, PhD thesis, University of Twente, Enschede, The Netherlands. ISBN: 978-90-365-2613-5.

Van der Veen, H. H., Hulscher, S. J. M. H. and Knaapen, M. A. F. [2006]. Grain size dependency in the occurence of sand waves, Ocean Dynamics 56: 228-234. doi: $10.1007 / \mathrm{s} 10236-005-0049-7$.

Van Dijk, T. A. G. P. and Egberts, P. J. P. [2008]. The variability of sand wave migration in the North Sea, in D. Parsons, T. Garlan and J. Best (eds), Proceedings of the 3rd international workshop on Marine Sand waves and River Dune Dynamics, Leeds (UK), University of Leeds, pp. 87-95.

Van Dijk, T. A. G. P. and Kleinhans, M. G. [2005]. Processes controlling the dynamics of compound sand waves in the North Sea, Netherlands, J. Geophys. Res. 110(F04S12). doi: 10.1029/2004JF000173.

Van Dijk, T. A. G. P., Lindenbergh, R. C. and Egberts, P. J. P. [2008]. Separating bathymetric data representing multiscale rhythmic bed forms: a geostatistical and spectral method compared, J. Geophys. Res. 113(F04017). doi: 10.1029/2007JF000950.

Van Maren, D. S. [1998]. Sand waves, a state-of-the-art review and bibliography, Master's thesis, Utrecht University, and North Sea Directorate, Ministry of Transport, Public Works and Water Management, Rijswijk, The Netherlands.

Van Munster, R. J., Van Antwerpen, G., Meulblok, M., Van Westenbrugge, C. J. and Borst, J. P. [1995]. DIGIPOL, intelligent interpolation of depth measurements, Proceedings of the Joint European Conference and Exhibition on Geographical Information, Vol. 1, JEC-GI, Basel, Switzerland, pp. 24-29.

Van Wijk, E. J. [2000]. Kan er inzicht worden verkregen in de bodemvariabiliteit van het POL-gebied voor de kust van den helder en kan daarmee bathymetrie voorspeld worden, Master's thesis, Royal Netherlands Naval College, Den Helder, The Netherlands.

Velberg, P. J. [1993]. The accuracy of the depth information of the nautical chart, Hydro. J. (68): 29-35. 
Vogelzang, J. [1997]. Mapping submarine sand waves with multiband imaging radar 1. model development and sensitivity analysis, J. Geophys. Res. 102(C1): 1163-1181.

Vogelzang, J., Wensink, G. J., Calkoen, C. J. and Van der Kooij, M. W. A. [1997]. Mapping submarine sand waves with multiband imaging radar 2. experimental results and model comparison, J. Geophys. Res. 102(C1): 1183-1192.

Wackernagel, H. [2003]. Multivariate geostatistics: an introduction with applications, 3rd edn, Springer-Verlag, Berlin, Germany.

Wells, D. E. and Monahan, D. [2002]. IHO S44 standards for hydrographic surveys and the variety of requirements for bathymetric data, Hydro. J. (104): 9-15.

Wever, T. F. [2004]. Bedforms and bedform migration: a data review, in S. J. M. H. Hulscher, T. Garlan and D. Idier (eds), Proceedings of the 2nd international workshop on Marine Sand Wave and River Dune Dynamics, Enschede, The Netherlands, University of Twente, Enschede, The Netherlands.

Whatrup, C., Mann, D. and Davidson, B. [2005]. The UK civil hydrography programme - changing the mould, Int. Hydro. Rev., New Series 6(2): 45-56.

Wright, P. [1992]. Long term changes to the positions and heights of sandwaves in the southern North Sea, Int. Hydro. Rev. 69(2): 113-128.

Wüst, J. C. [2004]. Data-driven probabilistic predictions of sand wave bathymetry, in S. J. M. H. Hulscher, T. Garlan and D. Idier (eds), Proceedings of the 2nd international workshop on Marine Sand Wave and River Dune Dynamics, Enschede, The Netherlands, University of Twente, Enschede, The Netherlands.

Wüst, J. C. [2005]. Images of trend analyses around the Eurogeul approach on CDROM. 


\section{Notation}

In the lists of symbols, all uppercase letters are given before lowercase letters. Within a case-group, roman typefaces are given before italics. The order within a case-group and a typeface-group is alphabetic, where the numbers are given after the letters. After the name of the symbol, we refer to the chapters in which it is explained further.

\section{Roman}

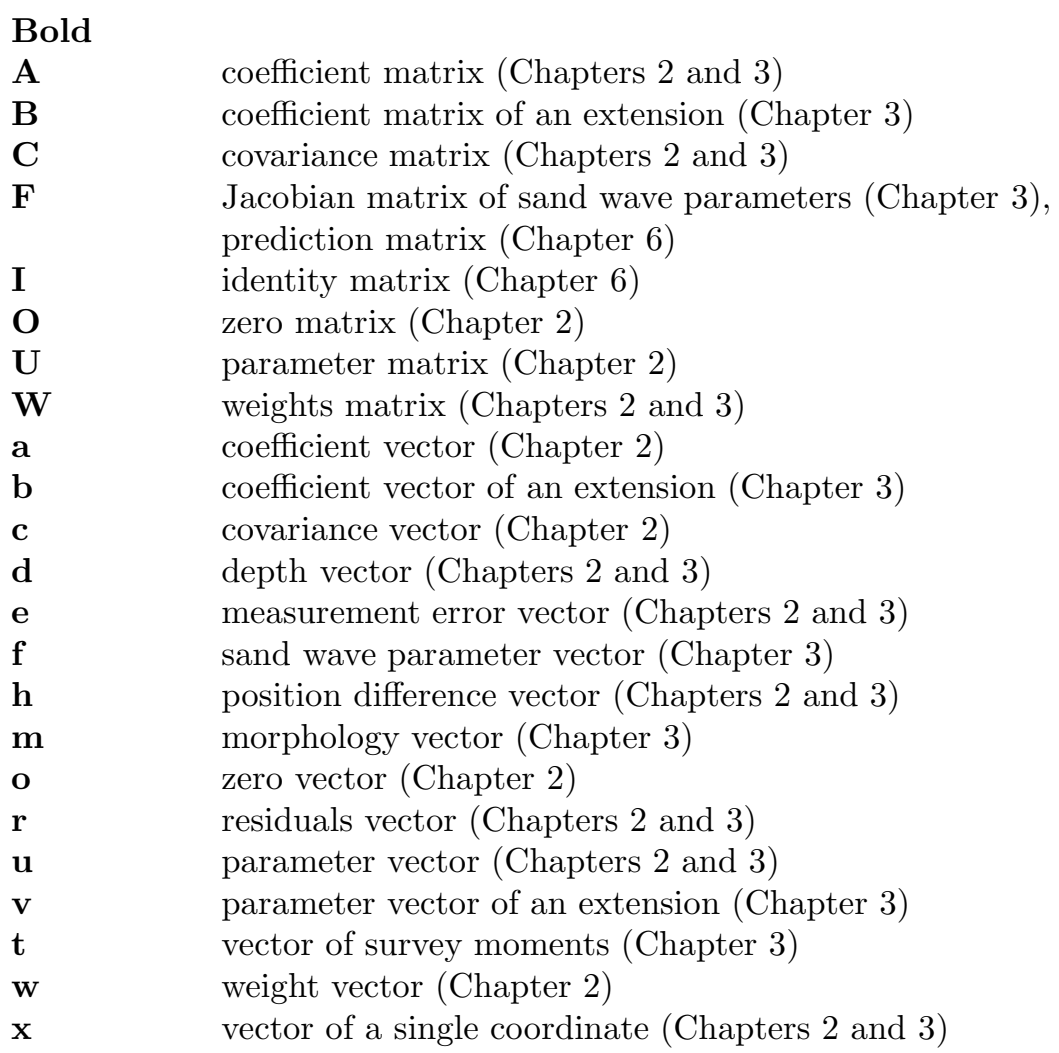




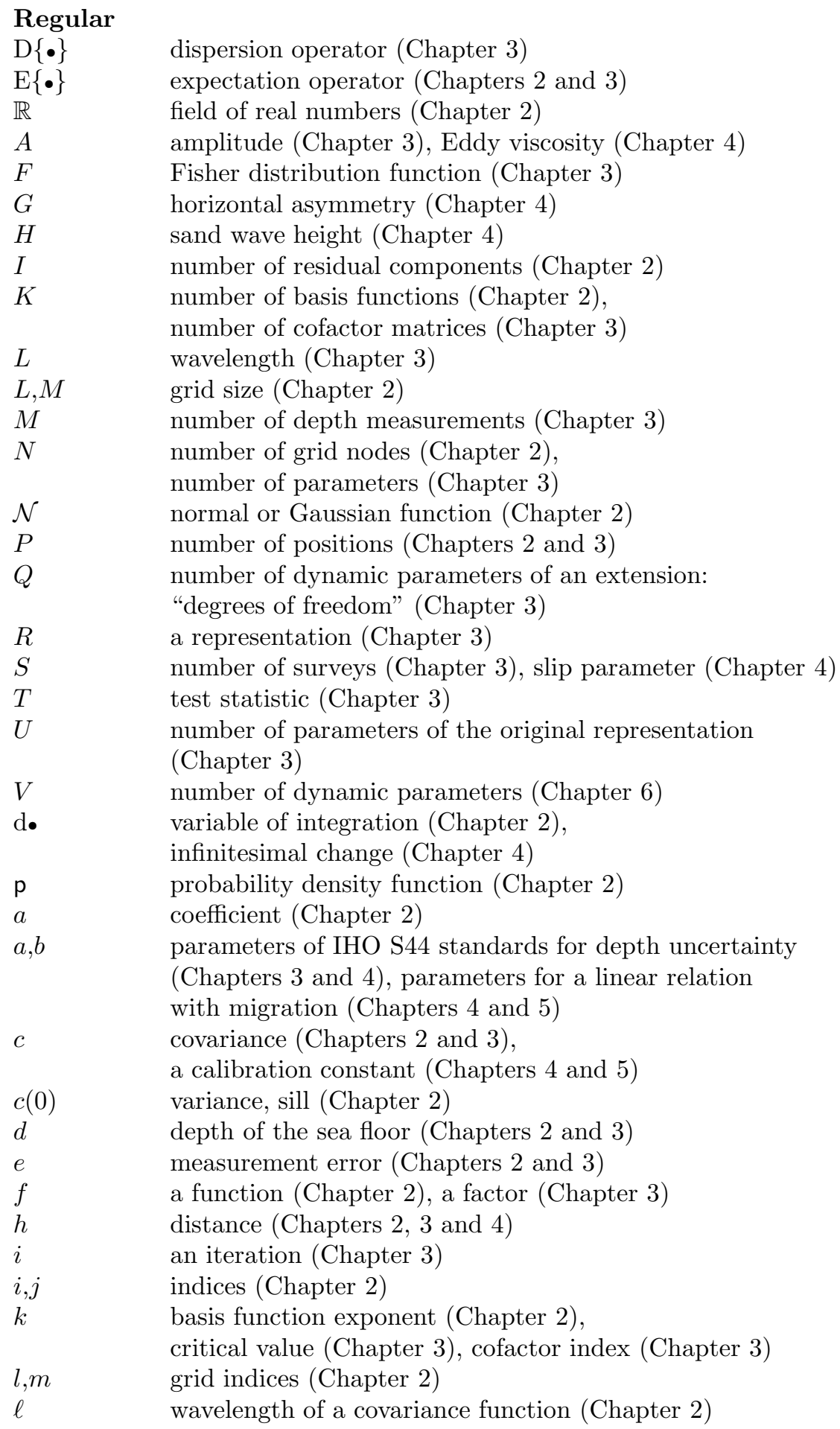


$m$

$p, q$

$q$

$r$

$t$

$u$

$w$

$x, y$

$z$

\section{Greek}

$\Delta \bullet$

$\Sigma \bullet$

$\alpha$

$\beta$

$\gamma$

$\gamma(0)$

$\theta$

$\vartheta$

$\kappa$

$\lambda$

$\xi$

$\pi$

$\rho$

$\sigma$

$\chi^{2}$

$\psi$

spatial trend (Chapters 2 and 3)

positions (Chapter 2)

test quotient (Chapter 3)

residual depth (Chapters 2 and 3 )

scale factor (Chapter 2)

a moment in time (Chapter 3)

parameter (Chapters 2, 3 and 4), flow velocity (Chapter 4)

variability (Chapter 2), parameter of an extension (Chapter 3), sound velocity (Chapter 4)

weight factor (Chapter 2)

horizontal directions (Chapters 2 and 3 )

vertical direction (Chapters 2 and 3 )

difference operator (Chapters 2 and 3 )

summation operator (Chapters 2 and 3 )

probability of incorrectly accepting an alternative

representation: "level of significance" (Chapter 3)

probability of incorrectly accepting the original

representation (Chapter 3)

variogram (Chapter 2),

probability of correctly accepting an alternative

representation: "power of a test" (Chapter 3)

nugget (Chapter 2)

azimuth East of North (Chapter 2)

azimuth increment (Chapter 2)

wave number (Chapters 2 and 3 )

noncentrality parameter (Chapter 3)

crest position (Chapter 3)

$3.14159268 \cdots($ Chapter 2)

correlation coefficient (Chapters 4,5 and 6)

standard deviation (Chapters 2, 3, 4 and 5)

chi-squared distribution function (Chapter 3)

slope (Chapter 3)

gradient (Chapter 2) 


\section{Augmentations}

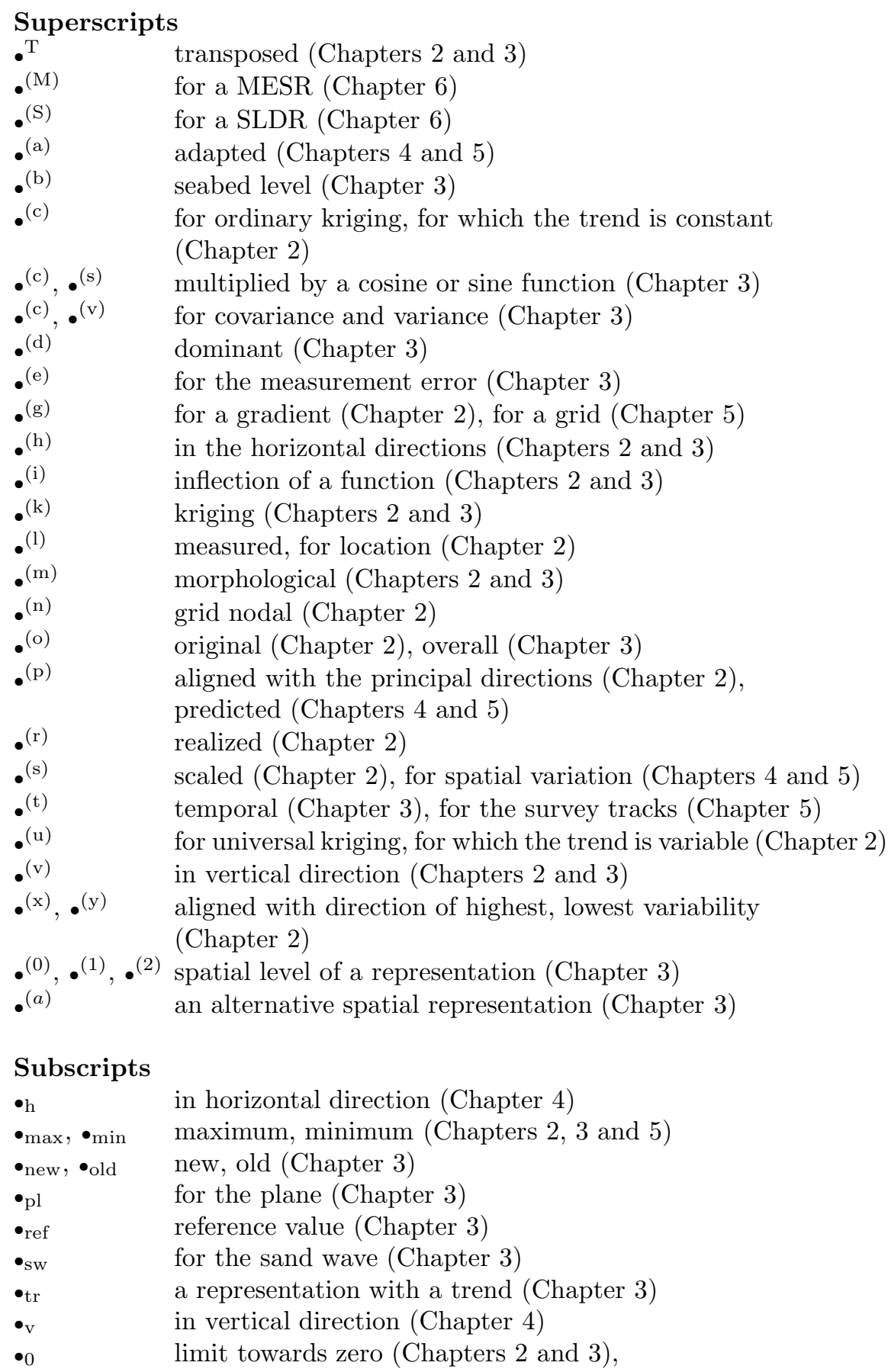


for the original temporal representation (Chapter 3)

-1 $\quad$ for one degree of freedom (Chapter 3)

$\bullet_{1}, \bullet_{2}, \cdots \quad$ for a specific value of a subscript

(Chapters 3, 4 and 6)

$\bullet 2.5 \%, \bullet 97.5 \%$ lower and upper limit of 95\% confidence interval (Chapter 6)

- $M$ with size equal to the number of measurements (Chapter 6)

- $P$ with size equal to the number of positions (Chapter 6)

- $S \quad$ for the last survey (Chapter 6)

- $S+1 \quad$ for expected next survey (Chapter 6$)$

- $a \quad$ an alternative temporal representation (Chapter 3)

-d $\quad$ for depth (Chapters 3, 4 and 5)

-2d to and from the sea floor (Chapter 4)

${ } i \quad$ residual component index (Chapter 2), iteration index (Chapter 3), variance component index (Chapter 4)

$\bullet \quad$ basis function index (Chapter 2), cofactor index (Chapter 3)

$\bullet_{m}, \bullet_{n} \quad$ a grid node (Chapter 2)

- $m$ for the morphological trend (Chapter 3)

$\bullet_{p}, \bullet_{q} \quad$ a position (Chapters 2 and 3 )

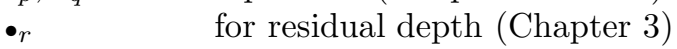

-s $\quad$ for a survey (Chapter 3)

- $u \quad$ for the parameters (Chapter 3)

$\bullet_{x}, \bullet_{y} \quad$ aligned with direction of highest, lowest variability (Chapters 3 and 4 )

- $\alpha \quad$ for a set level of significance (Chapter 3)

- $\xi \quad$ for migration (Chapters 4 and 5)

Other

\begin{tabular}{|c|c|}
\hline & $\begin{array}{l}\text { average (Chapters } 2 \text { and } 6) \\
\text { trend (Chapter } 3 \text { ) }\end{array}$ \\
\hline & least-squares estimate (Chapter 3) \\
\hline & within a specified interval (Chapter 2) \\
\hline & with respect to a reference value (Chapter 3 ) \\
\hline & extended with a value for the next survey (Chapter 6) \\
\hline & $\begin{array}{l}\text { in positive and negative direction of the } x \text {-axis (Chapter } 4 \text { ) } \\
\text { for an SBES survey (Chapter } 6 \text { ) }\end{array}$ \\
\hline & for an MBES survey (Chapter 6) \\
\hline & stochastic (Chapters 2 and 3 ) \\
\hline & function of a discrete signal (Chapter 2) \\
\hline & function of a continuous signal (Chapters 2 and 3 ) \\
\hline$\bullet \bullet \bullet\}$ & statistical operator of a variable (Chapters 2 and 3 ) \\
\hline$|\bullet|$ & magnitude operator (Chapters 2 and 3 ) \\
\hline & minimal detectable value (Chapter 3 ) \\
\hline
\end{tabular}




\title{
About the author
}

\begin{abstract}
Activities
Leendert Louis Dorst was born in IJsselstein, the Netherlands, on May 10, 1975. He attended the Katholieke Scholengemeenschap De Breul in Zeist, where he received his Gymnasium diploma in 1993. Then, he studied Geodetic Engineering at the Delft University of Technology. As part of his studies, he worked at the Finnish Geodetic Institute on projects related to post-glacial rebound in 1997, and at the Royal Observatory of Belgium on the analysis of time series of a superconducting gravimeter in 1999. Besides this study, he was active as a leader at Scouting group De Woudlopers in Zeist, as a board member at the Landmeetkundig Gezelschap Snellius, as an active member of the Delftse Studentenvereniging Sint Jansbrug, and as a student member of the education committee of Geodetic Engineering. In November 1999, he received his Master in Science.
\end{abstract}

In February 2000, Leendert Dorst started as an employee in the Department of Geodesy and Tides of the Hydrographic Service, part of the Royal Netherlands Navy, where he started to study the application of geostatistics and deformation analysis to a series of bathymetric surveys. This project continued as a cooperation with the Water Engineering and Management group of the University of Twente, resulting in his $\mathrm{PhD}$ defence in September 2009. He has supervised two MSc students on this subject, Peter Menting of the Delft University of Technology in 2004, and Bregt Huizenga of the University of Twente in 2007.

Since May 2008, Leendert Dorst has been employed as head of the Department of Geodesy and Tides, where he works on technical aspects of the law of the sea, advice on maritime positioning, statistical aspects of bathymetric surveying, and transformations between coordinate systems. He is a member of the management team of the Hydrographic Service, of the Subcommittee on Marine Geodesy of the Netherlands Geodetic Committee, and of the Working Groups on Standards for Hydrographic Surveys and on Data Quality of the International Hydrographic Organization. 


\section{Related publications}

2009: L. L. Dorst, P. C. Roos, F. M. Sterlini and S. J. M. H. Hulscher. The analysis of migrating tidal sand waves in an anchorage area on the Netherlands Continental Shelf, submitted.

L. L. Dorst, P. C. Roos, S. J. M. H. Hulscher and R. C. Lindenbergh. The Estimation of Sea Floor Dynamics from Bathymetric Surveys of a Sand Wave Area, J. of Applied Geodesy, 3(3), 97-120.

2008: L. L. Dorst, P. C. Roos, F. M. van der Meer and S. J. M. H. Hulscher. Sand wave migration in an anchorage area in the Southern North Sea. In: Proceedings of the third international workshop on Marine sandwave and River dune Dynamics, Leeds, United Kingdom, 2008. Edited by D. Parsons, T. Garlan and J. Best. ISBN 978-2-11-0972088.

2007: R. C. Lindenbergh, L. L. Dorst, J. C. Wüst and P. G. Menting. Simulation, detection and prediction of sea floor dynamics, Int. Hydro. Rev., New Series, 8(2), 25-36.

L. L. Dorst, P. C. Roos and S. J. M. H. Hulscher. Estimation of sand wave dynamics in the Southern North Sea. In: Proceedings of the thirtieth International Conference on Coastal Engineering, San Diego, USA, 2006. Edited by J. McKee Smith. ISBN: 13978981270 6362.

2006: P. G. Menting, L. L. Dorst, R. C. Lindenbergh and J. C. Wüst. Simulation, Detection and Prediction of Sea Floor Dynamics, In: Proceedings of Hydro06, Antwerp, Belgium, 2006.

L. L. Dorst and C. Howlett. Evolutions in the IHO S44 standards for hydrographic surveys, In: Proceedings of Hydro06, Antwerp, Belgium, 2006.

2005: L. L. Dorst. Optimization of survey frequency at the Netherlands Hydrographic Service, Hydro International, 9(8), 6-9.

L. L. Dorst, P.C. Roos and S.J.M.H. Hulscher. The estimation of various types of sea floor change. In: NCK four-year report 20012004 .

2004: L. L. Dorst. Detection of sea floor dynamics from depth data at the Netherlands Hydrographic Office, Int. Hydro. Rev., New Series, $5(2), 49-63$.

L. L. Dorst. More efficient and reliable sea floor mapping by geodetic interpretation of bathymetric data. In: Proceedings of the seventh European Conference on Underwater Acoustics, Delft, The Netherlands, 2004. Edited by D. G. Simons. ISBN: 90-5986-079-9.

L. L. Dorst. Geodetic deformation analysis: a new method for the estimation of seabed dynamics. In: Proceedings of the second international workshop on Marine sandwave and River dune Dynamics, Enschede, The Netherlands, 2004. Edited by S. J. M. H. Hulscher, T. Garlan and D. Idier. 